Copyright

by

Son Van Cao

2014 
The Dissertation Committee for Son Van Cao

certifies that this is the approved version of the following dissertation:

\title{
Study of antineutrino oscillations using accelerator and atmospheric data in MINOS
}

\author{
Committee: \\ Karol Lang, Supervisor \\ Duane Dicus \\ Jack Ritchie \\ Roy Schwitters \\ Philip Varghese
}




\title{
Study of antineutrino oscillations using accelerator and atmospheric data in MINOS
}

\author{
by
}

Son Van Cao, B.S.

\author{
DISSERTATION \\ Presented to the Faculty of the Graduate School of \\ The University of Texas at Austin \\ in Partial Fulfillment \\ of the Requirements \\ for the Degree of \\ DOCTOR OF PHILOSOPHY
}

THE UNIVERSITY OF TEXAS AT AUSTIN

May 2014 
Dedicated to my family. 


\section{Acknowledgments}

I wish to thank many people who have greatly helped and supported me. First, I would like to deeply thank my supervisor, Karol Lang, for his phenomenal patience, advice and guidance throughout research for this dis-

sertation. I sincerely hope to work with him again sometime. Thanks to the Vietnam Education Foundation for sponsoring me since I came to Texas.

I would like to thank the CC Disappearance Working Group conveners, Justin Evans, Alex Sousa, Mike Kordosky and Donna Napples for patiently educating me on the overall analysis framework and a number of relevant physics aspects. I also am in debt to Rustem Ospanov and Jasmine Ratchford for friendly sharing of their experience on event classification. Thanks to Chris Backhouse for putting up with me since I started working on the kNN shower energy estimator. I am especially thankful to Andy Blake for helping me on the analysis with a complete atmospheric and accelerator data set.

I would like to thank Rashid Mehdiyev for his friendship and sharing his expert knowledge on many things. Thanks to Robert Hatcher and Art Kreymer for providing valuable corrections and advices on programming and computing. Thanks to Benton Pahlka, Adam Shreckenberger, Junting Huang, Navaneeth Poonthottathil, Michelle Medeiros, Sharan Kalwani and Jessica Sands for correcting many things in the context of this dissertation.

Finally, I would like to thank my family for unconditionally supporting me. Especially, I thank my beautiful wife Trang Do, for being there. 


\title{
Study of antineutrino oscillations using accelerator and atmospheric data in MINOS
}

\author{
Publication No.
}

Son Van Cao, Ph.D.

The University of Texas at Austin, 2014

Supervisor: Karol Lang

The Main Injector Neutrino Oscillation Search (MINOS) is a long baseline experiment that was built for studying the neutrino oscillation phenomena. The MINOS experiment uses high intensity muon neutrino and antineutrino beams created by Neutrinos at the Main Injector facility (NuMI) at the Fermi National Accelerator Laboratory (Fermilab). Neutrino interactions are recorded by two sampling steel-scintillator tracking calorimeters: 0.98 kton Near Detector at Fermilab, IL and 5.4 kton Far Detector at the Soudan Underground Laboratory, MN. These two detectors are functionally identical, which helps to reduce the systematic uncertainties in the muon neutrino and antineutrino disappearance measurements. The Near Detector, located $1.04 \mathrm{~km}$ from the neutrino production target, is used to measure the initial beam composition and neutrino energy proximal to the neutrino source. The collected data at the Near Detector is then used to predict energy spectrum in the Far Detector. By comparing this prediction to collected data at the Far Detector, 
which is $735 \mathrm{~km}$ away from the target, it enables a measurement of a set of parameters that govern the neutrino oscillation phenomenon.

The flexibility of the NuMI beam configuration and the magnetization of the MINOS detectors facilitate the identification of $\nu_{\mu}$ and $\bar{\nu}_{\mu}$ chargedcurrent interactions on an event-by-event basis. This enables one to measure neutrino and antineutrino oscillation parameters independently and therefore allows us to test the CPT symmetry in the lepton sector. To enhance the sensitivity of the oscillation parameters measurement, a number of techniques have been implemented. Event classification, shower energy estimation and energy resolution bin fitting, which are described in this dissertation, are three of these techniques. Moreover, the most stringent constraints on oscillation parameters can be achieved by combining multiple data sets.

This dissertation reports the measurement of antineutrino oscillation parameters using the complete MINOS accelerator and atmospheric data set of charged-current $\bar{\nu}_{\mu}$ events. This set comprises exposures of (i) $3.36 \times$ $10^{20}$ proton-on-target $(\mathrm{POT})$ in the $\bar{\nu}_{\mu}$-beam mode, (ii) $10.71 \times 10^{20} \mathrm{POT}$ in the $\nu_{\mu}$-beam mode, and (iii) 37.88 kton yr of atmospheric antineutrinos. The data analysis provides the world's most precise measurement to date on the antineutrino oscillation parameters: $\left|\Delta \bar{m}^{2}\right|=\left(2.50_{-0.29}^{+0.23}\right) \times 10^{-3} \mathrm{eV}^{2}$ and $\sin ^{2}(2 \bar{\theta})=0.97_{-0.08}^{+0.03}$. This result is consistent with neutrino oscillation parameters independently measured by MINOS and by others. The difference between antineutrino and neutrino mass-squared splittings is computed to be $\left|\Delta \bar{m}^{2}\right|-\left|\Delta m^{2}\right|=\left(0.13_{-0.25}^{+0.23}\right) \times 10^{-3} e V^{2}$. 


\section{Table of Contents}

Acknowledgments $\quad$ v

Abstract vi vi vi vis

$\begin{array}{lll}\text { Chapter 1. Motivation and introduction } & 1\end{array}$

1.1 Motivation and outline of this dissertation . . . . . . . . . . 1

1.2 Overview of neutrino history . . . . . . . . . . . . 4

1.3 Electroweak unification and neutrinos . . . . . . . . . . 8

1.4 Neutrino oscillation phenomena . . . . . . . . . . . . . 11

1.4.1 Solar neutrino puzzle . . . . . . . . . . . . . . . . . . . 12

1.4.2 Atmospheric neutrino puzzle . . . . . . . . . . 13

1.4.3 Neutrino oscillation theory . . . . . . . . . . . . 14

1.4.4 Matter effect in neutrino oscillations . . . . . . . . . . 19

1.4.5 Antineutrino and neutrino oscillations . . . . . . . . . . . 23

1.4.6 Alternative disappearance models . . . . . . . . . 25

1.5 Outlook . . . . . . . . . . . . . . . . . 30

Chapter 2. The status of experimental neutrino oscillations 32

2.1 Natural neutrino source-based programs . . . . . . . . . . . . . 32

2.1.1 Cosmology and supernova neutrinos . . . . . . . . . . 32

2.1.2 Solar neutrinos . . . . . . . . . . . . . . . . 33

2.1.3 Atmospheric neutrinos . . . . . . . . . . . 35

2.2 Man-made neutrino source-based programs . . . . . . . . . . 37

2.2.1 Reactor neutrinos . . . . . . . . . . . . . . 37

2.2.2 Accelerator neutrinos . . . . . . . . . . . . 39

2.2.3 Future of neutrino beams . . . . . . . . . . . 43

2.3 Experimental status of neutrino mixing . . . . . . . . . . . 44

2.3.1 Solar sector . . . . . . . . . . . . . . . . . 44 
2.3.2 Atmospheric sector . . . . . . . . . . . . . . . 45

2.3.3 Tau neutrino appearance from muon neutrino beam . . 46

2.3 .4 Mixing angle $\theta_{13} \ldots \ldots \ldots \ldots \ldots$

2.3.5 Sterile neutrinos . . . . . . . . . . . . . 48

2.4 Experimental status of absolute neutrino mass . . . . . . . 50

2.4.1 Supernovae and cosmological constraints . . . . . . . 50

2.4 .2 Neutrinoless double beta decay . . . . . . . . . . . 51

2.5 Experimental status of neutrino velocity . . . . . . . . 51

2.6 Future prospects of neutrino oscillation experiments . . . . . 53

$\begin{array}{lll}\text { Chapter 3. The MINOS experiment } & 56\end{array}$

3.1 Accelerator-based neutrino experiments . . . . . . . . 56

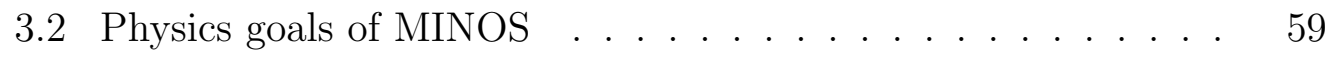

3.3 The NuMI neutrino beam . . . . . . . . . . . . . . . 61

3.4 Detector technology . . . . . . . . . . . . . 70

3.4.1 Detector overview . . . . . . . . . . . . 70

3.4 .2 Steel and magnetization . . . . . . . . . . . . 77

3.4.3 Scintillator and module design . . . . . . . . . 78

3.4 .4 PMT and enclosures . . . . . . . . . . . . . 80

3.4 .5 Electronics and $\mathrm{DAQ} \ldots \ldots \ldots 1 \ldots$

3.5 Signal calibration . . . . . . . . . . . . . . . 82

3.6 Monte Carlo simulation . . . . . . . . . . . . . . 86

3.7 Event reconstruction . . . . . . . . . . . . . . . . . 87

3.8 The MINOS+ experiment . . . . . . . . . . . . . 93

Chapter 4. The event classification in MINOS 95

4.1 Event topologies . . . . . . . . . . . . . . . . . . . . 95

4.2 Muon charge-sign measurement . . . . . . . . . . . . . 100

4.3 Muon and non-muon track separation . . . . . . . . . . 102

4.3.1 Topological features of muon tracks . . . . . . . . . . 104

4.3.2 The k-nearest neighbors algorithm for muon track identification . . . . . . . . . . . . . 110

4.3.3 Results of muon track identification . . . . . . . . . . 114 
4.4 Charged-current $\bar{\nu}_{\mu}$ event selection . . . . . . . . . . . . 117

4.4.1 Selecting charged-current $\bar{\nu}_{\mu}$ events in the $\bar{\nu}_{\mu}$-beam . . . 117

4.4.2 Selecting charged-current $\bar{\nu}_{\mu}$ events in the $\nu_{\mu}$-beam . . . 119

4.4.3 Improving the beam charged-current $\bar{\nu}_{\mu}$ event selection . 121

4.4.4 Selecting atmospheric charged-current $\bar{\nu}_{\mu}$ events . . . . . 127

4.5 Neutral-current event selection . . . . . . . . . . . . 132

4.5 .1 Preselection . . . . . . . . . . . . . . . . . 132

4.5 .2 Main selection . . . . . . . . . . . . . . . . . . 133

4.5.3 Improving the neutral-current selection . . . . . . . . 134

4.6 Summary of event classification . . . . . . . . . . . 137

Chapter 5. The hadronic shower energy estimator 138

5.1 Motivation for hadronic shower energy estimator . . . . . . . 138

5.2 Shower characteristics . . . . . . . . . . . . . . . . . 141

5.3 Regression analysis for shower energy estimator . . . . . . . . 142

$5.4 \mathrm{kNN}$ shower energy estimator . . . . . . . . . . . . . . 144

5.5 Systematic uncertainties of the kNN shower energy estimator . 154

5.5.1 Uncertainties due to detector calibration . . . . . . . 155

5.5.2 Uncertainties due to hadronization modeling . . . . . . 156

5.5.3 Uncertainties due to intranuclear rescattering . . . . . . 159

5.5.4 Total combined systematics . . . . . . . . . . . . 162

5.6 Summary of hadronic shower energy estimator . . . . . . . 163

\section{Chapter 6. Analysis of accelerator charged-current muon an- ${ }_{164}$}

6.1 Accelerator charged-current $\bar{\nu}_{\mu}$ data preselection . . . . . . 165

6.2 Accelerator charged-current $\bar{\nu}_{\mu}$ data selection . . . . . . . 169

6.3 The $\bar{\nu}_{\mu}$-beam flux reweighting $\ldots \ldots \ldots \ldots \ldots$

6.4 Far Detector prediction . . . . . . . . . . . . . . . 174

6.5 Systematic error evaluation . . . . . . . . . . 180

6.6 Oscillation parameter fitting . . . . . . . . . . . . 186

6.7 Summary of the accelerator charged-current $\bar{\nu}_{\mu}$ disappearance analysis . . . . . . . . . . . . . . . . . 188 
Chapter 7. Results of the MINOS $\bar{\nu}_{\mu}$ charged-current disappearance analyses $\quad 189$

7.1 The complete MINOS $\bar{\nu}_{\mu}$ data set $\ldots \ldots \ldots \ldots$

7.2 Measurements from the $\bar{\nu}_{\mu}$-beam data $\ldots \ldots \ldots . \ldots . . \ldots 191$

7.3 Measurements from both $\bar{\nu}_{\mu}$-beam and $\nu_{\mu}$-beam data $\ldots . .200$

7.4 Measurements from combined accelerator and atmospheric data 206

7.5 CPT-invariant testing in $\nu_{\mu}$ and $\bar{\nu}_{\mu}$ oscillations . . . . . 215

7.6 Comparison to MINOS results . . . . . . . . . . . . . . . . 219

7.7 Discussion of dissertation results . . . . . . . . . . . . 222

Chapter 8. Summary and outlook $\quad 224$

8.1 Summary of this dissertation . . . . . . . . . . . . . . 224

8.2 Outlook on the antineutrino oscillations measurements . . . 225

8.3 Conclusion . . . . . . . . . . . . . . . . . 227

$\begin{array}{ll}\text { Appendices } & 228\end{array}$

Appendix A. Degeneracy and correlation among oscillation parameters

Appendix B. The SKZP reweighting effect on oscillation parameters

Appendix C. Fitting in bins of energy resolution 236

Appendix D. Mass hierarchy resolvability with long-baseline experiments

Appendix E. Auxiliary Detector and measurement of neutrino velocity in MINOS 241

E.1 Motivations . . . . . . . . . . . . . . . . . . . . . 241

E.2 Auxiliary Detector and readout system . . . . . . . . . . . 244

E.3 Matching muons between the AD and MINOS detector . . . . 246

E.4 Results of latency measurements . . . . . . . . . . . . . . 251

E.5 Results of neutrino velocity measurement . . . . . . . . 251

E.6 Summary . . . . . . . . . . . . . . . . . . . . . . . 257 
$\begin{array}{lll}\text { Appendix F. } & \text { Unitarity of the PMNS matrix } & 258\end{array}$

$\begin{array}{ll}\text { Bibliography } & 262\end{array}$

$\begin{array}{ll}\text { Vita } & 280\end{array}$ 


\section{Chapter 1}

\section{Motivation and introduction}

\subsection{Motivation and outline of this dissertation}

Since their initial invention in the 1930s [1], neutrinos have emerged as some of the most interesting elementary particles. Neutrino oscillation (discussed in Chapter 1), now a well-understood phenomenon, indicates that neutrinos have mass, and their flavor eigenstates are different from their mass eigenstates. Neutrino oscillations provide the first evidence of physics beyond the Standard Model of the elementary particles. Over eight decades, our picture of neutrino physics has been revolutionized, but some fundamental questions still remain unanswered (as exposed in Chapter 2).

The study of neutrino oscillations allows one to examine a number of fundamental properties. Charge, parity and time reversal (CPT) invariance, a fundamental principle in quantum field theory, requires that particles and anti-particles share certain properties, including charge and mass. Neutrino oscillation measurement cannot tell us the absolute neutrino mass scale, $m_{i}$, but only the mass-squared splitting $\Delta m_{i j}^{2}=m_{i}^{2}-m_{j}^{2}$. Violation of the CPT symmetry could manifest itself in neutrino oscillations as the difference of mass-squared splittings for neutrinos and antineutrinos (i.e., $\Delta m_{i j}^{2} \neq \Delta \bar{m}_{i j}^{2}$ ). This is the central issue of this dissertation. 
MINOS detectors (described in Chapter 3) are magnetized tracking calorimeters and therefore enable separate charged-current $\nu_{\mu}$ and $\bar{\nu}_{\mu}$ interactions on an event-by-event basis, giving a unique opportunity to measure the neutrino and antineutrino oscillations simultaneously. Also, the flexibility of NuMI beam allows a $\bar{\nu}_{\mu}$ beam to statistically increase a number of observed $\bar{\nu}_{\mu}$ events, which normally lag well behind the number of observed $\nu_{\mu}$ events. Therefore, measurement of $\bar{\nu}_{\mu}$ disappearance in the MINOS Far Detector, as compared to the prediction from the Near Detector, not only enriches our knowledge of antineutrino oscillations but provides a test of the CPT invariance in the lepton sector.

In 2010, MINOS reported the measurement of antineutrino oscillation parameters from the direct observation of antineutrino muon disappearance [2]. The $\bar{\nu}_{\mu}$-CC data from an exposure of $1.71 \times 10^{20}$ protons on target (POT) in the $\bar{\nu}_{\mu}$-beam mode agreed with the effective two-flavor oscillation hypothesis with parameters $\Delta \bar{m}^{2}=3.36_{-0.40}^{+0.46}$ (stat) \pm 0.06 (syst) $\times 10^{-3} \mathrm{eV}^{2}$ and $\sin ^{2}(2 \bar{\theta})=0.86 \pm 0.11$ (stat) \pm 0.01 (syst). Assuming that neutrino and antineutrino oscillations are governed by an independent set of oscillation parameters, the $\bar{\nu}_{\mu}$ and $\nu_{\mu}$ measurements are consistent at the $2 \%$ confident level. Figure 1.1 shows the comparison of $90 \%$ C.L. allowed regions for neutrino and antineutrino oscillation parameter measured by MINOS. The measurement reported a tension between the underlying mechanisms of neutrino and antineutrino oscillations, but was insufficient to warrant evidence of CPT violation or a requirement of introducing non-standard particles or interactions. 


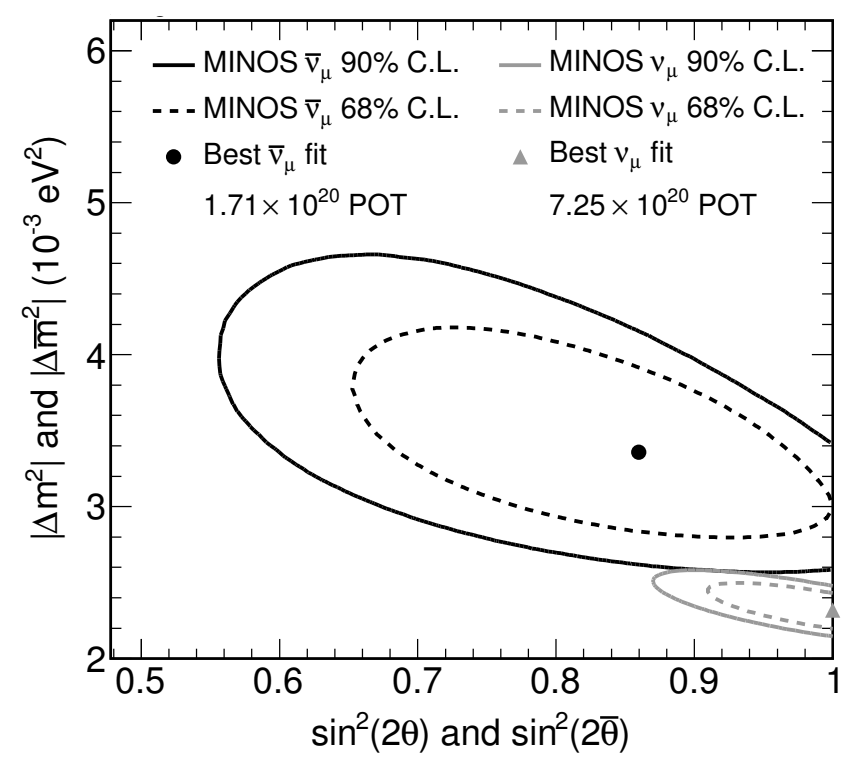

Figure 1.1: The allowed regions from the independent measurements of $\nu_{\mu}$ and $\bar{\nu}_{\mu}$ oscillation parameter. Figure taken from [2].

This measurement was limited by statistics. Since then, MINOS has collected an additional $1.65 \times 10^{20} \mathrm{POT}$ in the $\bar{\nu}_{\mu}$-beam mode to enhance the measurement of antineutrino oscillation parameters. In addition, a number of techniques such as event classification (discussed in Chapter 4) and hadronic shower energy (described in Chapter 5) have been developed to improve the sensitivity to antineutrino oscillation parameters. A software framework for utilizing two-detector design of the MINOS experiment (introduced in Chapter 6 ) is used to mitigate the systematic uncertainties. Also, this dissertation presents the first joint measurement of the complete accelerator and atmospheric data (shown in Chapter 7). All these aims to achieve the world's 
most precise measurement of antineutrino oscillation parameters and clarify ambiguity in previously reported results by MINOS. Figure 1.2 highlights the ultimate results of antineutrino oscillation parameters, which are presented in this dissertation. Finally, Chapter 8 summarizes the results of this dissertation and presents an outlook on the forthcoming antineutrino oscillation measurements.
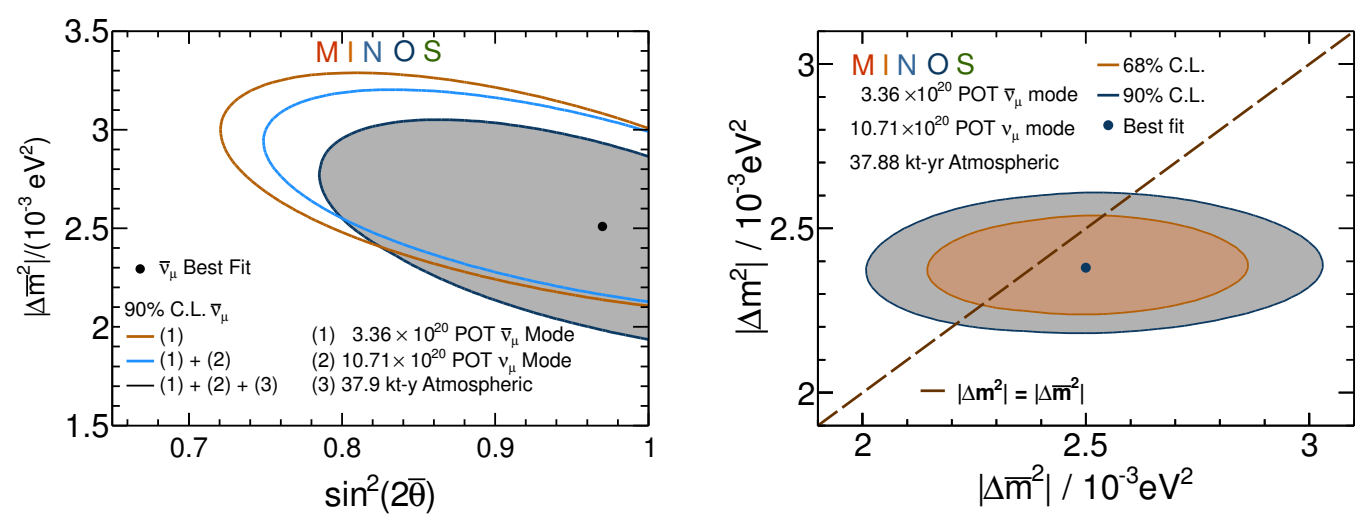

Figure 1.2: The left plot shows the progression of $90 \%$ confident level (C.L.) surfaces of antineutrino oscillation parameters by adding more data sets. The right plot shows the agreement between the neutrino and antineutrino masssquared splittings.

\subsection{Overview of neutrino history}

In 1914, James Chadwick [3] discovered that the spectrum of electrons emitted in $\beta$-decays was continuous. This discovery was contrary to the prescription of quantum mechanics, in which the spectrum of emitted electrons was predicted to be discrete due to the law of energy conservation. As a "desperate" remedy to escape this dilemma and preserve the conservation of 
energy, W. Pauli introduced a new neutral particle with spin of $1 / 2$, named the neutron ${ }^{1}$, in a letter sent to the Physical Society of Tübingen in Zurich in $1930[1]:$

"I admit that my way out may look rather improbable at first since if the neutron existed it would have been seen long ago. But nothing ventured, nothing gained."

Much has changed since then. In 1956, Reines and Cowan [4] discovered experimentally the first neutrinos by creating electron antineutrinos, $\bar{\nu}_{e}$, from beta decay in nuclear reactors, which collided with protons to produce nucleons and positrons. These two authors were then awarded the Nobel prize for this important discovery in 1995. Furthermore, in 1962, an experiment at Brookhaven lab [5] discovered that neutrinos, which were created in the pion decays, interacted with a detector and produced muons, but not electrons. This indicated the existence of the second generation of neutrinos, which was then experimentally confirmed by Lederman, Schwartz and Steinberger [6]. They received the Nobel prize shortly after claiming discovery of muon neutrinos, $\nu_{\mu}$, in 1988. In 1975, a new kind of event,

$$
e^{+}+e^{-} \rightarrow e^{ \pm}+\mu^{\mp}+\text { missing energy }
$$

was observed at the Stanford Linear Accelerator Center (SLAC) [7]. This was the first evidence of the third lepton generation, which is now known as the tau

\footnotetext{
${ }^{1}$ renamed to neutrino later by Fermi in 1931.
} 
lepton. The existence of tau neutrinos, $\nu_{\tau}$, was promptly conceived. In 2000, the Direct Observation of the NU Tau (DONUT) experiment at Fermilab [8] reported the first direct evidence of tau neutrinos.

Three flavors of neutrinos, equal to the number of observable lepton generations, have been confirmed and are widely accepted in the Standard Neutrino Model $(\mathrm{S} \nu \mathrm{M})$ [9]. The constraints on the number of neutrino flavors mainly come from the studies of $\mathrm{Z}$ boson decays [10] and the cosmological data [11]. Fourth and further neutrino flavors are considered to be unlikely. However, no direct proof for the exact three flavor paradigm is obvious and physicists maintain a skeptical view on existence of non-standard neutrinos.

In the neutrino history timeline, summarized in Figure 1.3, verifying the existence of neutrino oscillations, which was first proposed by Pontecorvo in 1957 [12], was of central importance. It took more than 40 years to discover this phenomenon. In 1998, the Super-Kamiokande (Super-K) collaboration [13] reported an evidence of neutrino oscillations in the atmospheric sector after analyzing more than 500 days of data. This evidence was then confirmed by the MINOS experiment [14] in 2006. Shortly after the SuperK's claim, solar neutrino oscillations were detected by the Sudbury Neutrino Observatory (SNO) experiment [15] in 2000 and confirmed by the Kamioka Liquid Scintillator Antineutrino Detector (KamLAND) [16] in 2002. The neutrino oscillations tell us that neutrinos have mass and thus provide the first experimental evidence of physics beyond the Standard Mode.

The neutrino oscillations are widely modeled by the mixing matrix be- 
tween flavor and mass eigenstate of neutrinos. This matrix is parameterized by three mixing angles and one $\mathrm{CP}$-violation phase. The last unknown mixing angle $\theta_{13}$, has recently been uncovered to be non-zero at a level of 7.7 sigma by the Daya Bay experiment [17] and of 7.3 sigma by the Tokai to Kamioka (T2K) experiment [18]. The precise measurement of this mixing angle is crucial since it allows us to measure the CP-violation phase in the lepton sector, which might relate to the dominance of matter over anti-matter in the present universe [19].

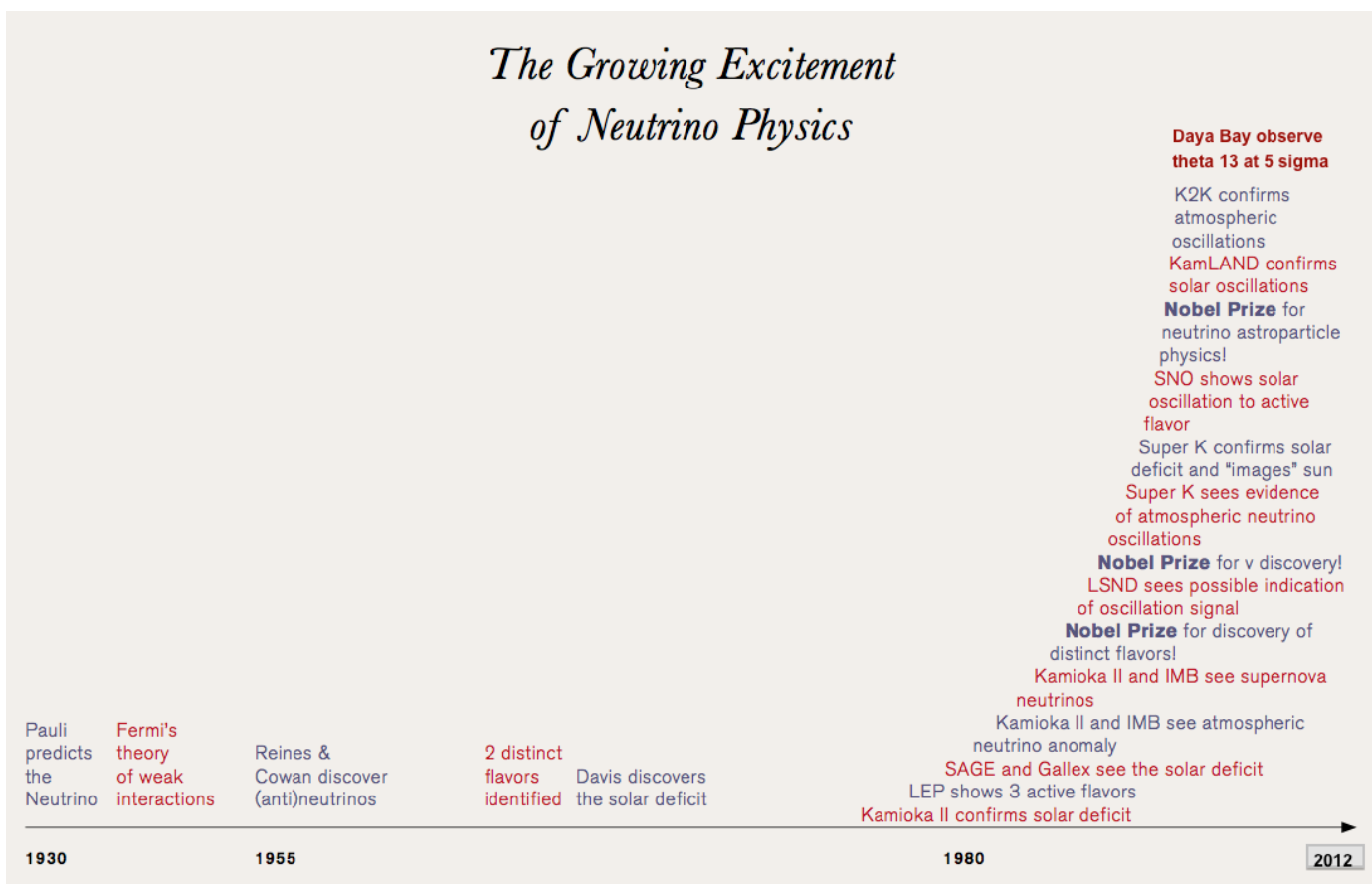

Figure 1.3: The growing excitement of neutrino physics. This shows the milestones of neutrino physics from the beginning until present. This taken from [20] and updated with Daya Bay observation. 
Another missing piece to complete the picture of neutrino oscillation is the order of neutrino masses. The values of mass-squared splittings are well determined but the hierarchy of neutrino absolute mass is still unknown. If the mass hierarchy is inverted, it would be the first observation that neutrinos are not typical fermions, which have increasing masses with increasing generation numbers. In the next few decades, answering these questions will be necessary to understand the nature of neutrinos, which has played a critical role at the frontier of physics, cosmology and astrophysics. All of this makes neutrino physics an exciting field.

\subsection{Electroweak unification and neutrinos}

Other than gravitational interactions, neutrinos only interact through weak nuclear force. The neutrino theory firstly developed by Fermi describes beta decay via a four-point interaction [21]. This theory predicts that the cross-section for the neutrino-nucleon scattering is proportional to the square of neutrino energy, which violates unitarity at around $300 \mathrm{GeV}[22]$. The most widely accepted theory of elementary particles is the Standard Model (SM), which was developed by Salam [23], Glashow [24] and Weinberg [25] based on the local gauge groups $\mathrm{SU}(3)_{\mathrm{C}} \times \mathrm{SU}(2)_{\mathrm{L}} \times \mathrm{U}(1)_{\mathrm{Y}}{ }^{2}$. In this theory, the lefthanded neutrino is grouped with the electron to form the presentation of the gauge group $\mathrm{SU}(2)_{\mathrm{L}}$, known as the electroweak unified theory.

\footnotetext{
${ }^{2}$ Subscripts C, L and Y are abbreviated for color charge, left-handed and weak hypercharge, respectively.
} 
The $\mathrm{SU}(2)_{\mathrm{L}} \times \mathrm{U}(1)_{\mathrm{Y}}$ group-based model for unified electroweak interaction was first introduced by Glashow in 1961 [24]. The left-handed electron, $e_{\mathrm{L}}$, and left-handed neutrino, $\nu_{\mathrm{L}}$, form a doublet, $d_{\mathrm{L}}=\left(\begin{array}{l}e \\ \nu\end{array}\right)_{\mathrm{L}}$, which is invariant under the weak isospin $\mathrm{SU}(2)_{\mathrm{L}}$ transformation. On the other hand, the righthanded electron, $e_{\mathrm{R}}$, stands as a singlet and invariant under the $\mathrm{U}(1)_{\mathrm{Y}}$ group. To build the Lagrangian for this model, three differential operators, $A_{\mu}^{a}$ with $a=1,2,3$, for the $\mathrm{SU}(2)_{\mathrm{L}}$ group and one $B_{\mu}$ for the $\mathrm{U}(1)_{\mathrm{Y}}$ group are needed. The infinitesimal gauge transformations for this model are given by:

$$
\begin{aligned}
& e_{\mathrm{R}} \rightarrow e^{i g^{\prime} B_{\mu}} e_{\mathrm{R}}, \\
& d_{\mathrm{L}} \rightarrow e^{-i \frac{g^{\prime}}{2} B_{\mu}+i \frac{g}{2} \tau_{a} A_{\mu}^{a}} d_{\mathrm{L}},
\end{aligned}
$$

where $\tau^{a}=\left(\tau^{1}, \tau^{2}, \tau^{3}\right)$ are the three Pauli matrices, $g$ and $g^{\prime}$ are two independent coupling constants associated with the group $\mathrm{SU}(2)_{\mathrm{L}}$ and $\mathrm{U}(1)_{\mathrm{Y}}$ respectively. To preserve the local gauge invariance, field derivatives need to be modified as follows:

$$
\begin{aligned}
& \not D e_{\mathrm{R}}=\left(\partial_{\mu}-i g^{\prime} B_{\mu}\right) e_{\mathrm{R}} \\
& \not D d_{\mathrm{L}}=\left(\partial_{\mu}+i \frac{g^{\prime}}{2} B_{\mu}-i \frac{g}{2} \tau_{a} A_{\mu}^{a}\right) d_{\mathrm{L}} .
\end{aligned}
$$

The total Lagrangian for the standard electroweak model is expressed as:

$$
\mathcal{L}_{\text {EW }}=\mathcal{L}_{\text {Lepton }}+\mathcal{L}_{\text {Gauge }}+\mathcal{L}_{\text {Higgs }}+\mathcal{L}_{\text {Yukawa }}
$$


where

$$
\begin{aligned}
\mathcal{L}_{\text {Lepton }} & =\overline{d_{\mathrm{L}}} i \not D d_{L} \mathrm{~L}+\overline{e_{\mathrm{R}}} i \not D e_{\mathrm{L}}, \\
\mathcal{L}_{\text {Gauge }} & =-\frac{1}{4} F_{\mu \nu}^{i} F^{i \mu \nu}-\frac{1}{4} B_{\mu \nu} B^{\mu \nu}, \\
\mathcal{L}_{\text {Higgs }} & =\left(D^{\mu} \phi\right)^{\dagger} D_{\mu} \phi-V(\phi), \\
\mathcal{L}_{\text {Yukawa }} & =f_{e}\left(\overline{e_{\mathrm{R}}} \phi^{+} d_{\mathrm{L}}+\overline{d_{\mathrm{L}}} \phi e_{\mathrm{R}}\right) .
\end{aligned}
$$

Here $F_{\mu \nu}^{i}=\partial_{\mu} A_{\nu}^{i}-\partial_{\nu} A_{\mu}^{i}$ and $B_{\mu \nu}=\partial_{\mu} B_{\nu}-\partial_{\nu} B_{\mu}$ are gauge fields; $\phi$ is the Higgs field. We define a field $W^{\mu}$ which annihilates $W^{+}$bosons and creates $W^{-}$bosons and rotate the $\left(A_{\mu}^{3}, B_{\mu}\right)$ plane to get a vector boson field $Z_{\mu}[26]$ :

$$
\begin{aligned}
W^{\mu} & =\frac{A_{1}^{\mu}-i A_{2}^{\mu}}{\sqrt{2}}, \\
Z^{\mu} & =\cos \vartheta_{W} A_{3}^{\mu}-\sin \vartheta_{W} B^{\mu}, \\
A^{\mu} & =\sin \vartheta_{W} A_{3}^{\mu}+\cos \vartheta_{W} B^{\mu},
\end{aligned}
$$

where $\vartheta_{W}$ is the weak mixing angle ${ }^{3}$. The lepton term in Eq. (1.4) is then separated into the charged-current $(\mathrm{CC})$ and neutral-current $(\mathrm{NC})$ interaction terms, $\mathcal{L}_{\text {Lepton }}=\mathcal{L}_{\mathrm{CC}}+\mathcal{L}_{\mathrm{NC}}$, where:

$$
\begin{aligned}
\mathcal{L}_{\mathrm{CC}}= & -\frac{g}{2 \sqrt{2}} j_{\mathrm{W}, \mathrm{L}}^{\mu} W_{\mu}+\text { h.c } \\
= & -\frac{g}{2 \sqrt{2}} v_{e L} \gamma^{\mu}\left(1-\gamma^{5}\right) e W_{\mu}+\text { h.c }, \\
\mathcal{L}_{\mathrm{NC}}= & -\frac{g}{2 \cos \vartheta_{W}} j_{\mathrm{Z}, \mathrm{L}}^{\mu} Z_{\mu}-e j_{\gamma, \mathrm{L}}^{\mu} A_{\mu} \\
= & -\frac{g}{2 \cos \vartheta_{W}}\left(\overline{\nu_{e}} \gamma^{\mu}\left(g_{V}^{\nu}-g_{A}^{\nu} \gamma^{5}\right) \nu_{e}+\bar{e} \gamma^{\mu}\left(g_{V}^{l}-g_{A}^{l} \gamma^{5}\right) e\right) Z_{\mu} \\
& -g_{e} \bar{e} \gamma^{\mu} e A_{\mu} .
\end{aligned}
$$

\footnotetext{
${ }^{3}$ also called as the Weinberg angle.
} 
Here $g_{e}=\sqrt{g^{2}+g^{\prime 2}}$ is the elementary electric charge.

The mass of electrons is given via the mechanism of spontaneous symmetry breaking of the $\mathrm{SU}(2)_{\mathrm{L}} \times \mathrm{U}(1)_{\mathrm{Y}}$ group to the $\mathrm{U}(1)_{\mathrm{EM}}{ }^{4}$ group when exciting the Higgs field above the vacuum:

$$
\phi(x)=\frac{1}{\sqrt{2}}\left(\begin{array}{c}
0 \\
\nu+H(x)
\end{array}\right) .
$$

The Higgs-lepton Yukawa coupling in Eq. (1.4) turns into:

$$
\mathcal{L}_{\text {Yukawa }}=-f_{e} \frac{v}{\sqrt{2}}\left(\overline{e_{\mathrm{L}}} e_{\mathrm{L}}+\overline{e_{\mathrm{R}}} e_{\mathrm{R}}\right)
$$

which gives the mass of electrons as $m_{e_{\mathrm{L}}}=m_{e_{\mathrm{L}}}=m_{e}=f_{e} \frac{v}{\sqrt{2}}$ and zero-mass for neutrinos. The SM predicts the masslessness of neutrinos. The backbone for this argument is the absence of right-handed neutrinos, which prohibit the interaction of left-handed neutrinos with Higgs bosons. However, this argument is only valid when considering the mass term as a Dirac mass term. The left-handed neutrinos by themselves can form a Majorana mass term [27], which violates the lepton number conservation by 2 units. In any case, the massiveness of neutrinos implies physics beyond the SM.

\subsection{Neutrino oscillation phenomena}

This section discusses the observation of the solar and atmospheric neutrino puzzles as well as the development of the neutrino oscillation theory and how it helps to explain these puzzles.

\footnotetext{
${ }^{4}$ Abbreviated for electromagnetic.
} 


\subsubsection{Solar neutrino puzzle}

The Sun is powered by nuclear fusion reactions happening in its core. These reactions provides a pure source of electron neutrinos, $\nu_{e} \mathrm{~s}$. The structure and dynamics of the solar core are modeled from the Standard Solar Model (SSM) [28]. The deficit of $\nu_{e} \mathrm{~s}$ in the neutrino flux from the Sun in comparison to the SSM, which was observed in many experiments such as Homestake $^{5}, \mathrm{GALLEX}^{6}, \mathrm{GNO}^{7}, \mathrm{SAGE}^{8}, \mathrm{SNO}^{9}, \mathrm{Kamiokande}$ and Super-Kamiokande (Super-K) [29-35], shown in Figure 1.4, is known as the solar neutrino anomaly.

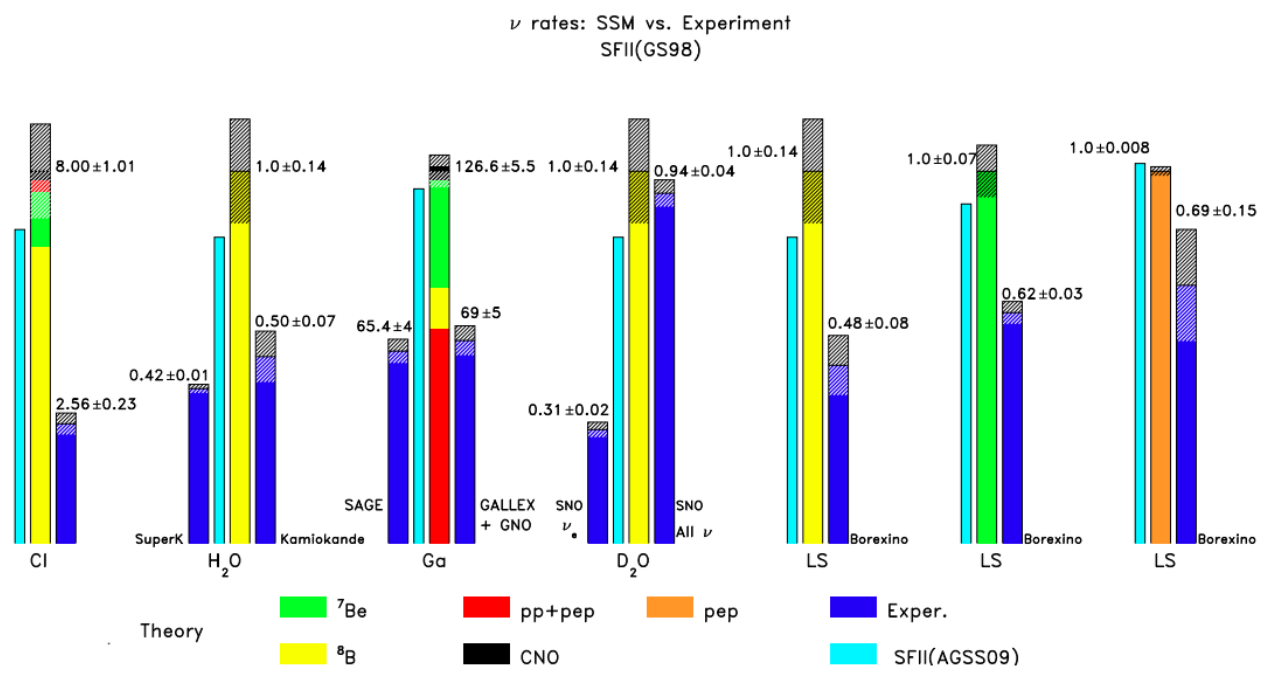

Figure 1.4: The ratios of measured solar neutrino fluxes from a variety of experiments to the SSM predictions. Figure taken from [36].

\footnotetext{
${ }^{5}$ Homestake is the radiochemical experiment at Homestake Gold Mine, South Dakota.

${ }^{6}$ GALLEX abbreviated for Gallium Experiment at Laboratori Nazionali del Gran Sasso.

${ }^{7}$ GNO abbreviated for Gallium Neutrino Observatory (GALLEX's successor.)

${ }^{8} \mathrm{SAGE}$ abbreviated for Soviet - American Gallium Experiment.

${ }^{9} \mathrm{SNO}$ abbreviated for Sudbury Neutrino Observatory.
} 


\subsubsection{Atmospheric neutrino puzzle}

High energy cosmic rays interacting with the Earth's atmosphere produce a cascade of pions, $\pi^{ \pm}$, and kaons, $K^{ \pm}$. These mesons, in turn, decay into neutrinos and antineutrinos via a number of processes as follows:

$$
\begin{aligned}
& p / H e+N \rightarrow X+\pi^{ \pm} / K^{ \pm}, \\
& \pi^{ \pm} / K^{ \pm} \rightarrow \mu^{ \pm}+\nu_{\mu}\left(\bar{\nu}_{\mu}\right), \\
& \mu^{ \pm} \rightarrow e^{ \pm}+\nu_{e}\left(\bar{\nu}_{e}\right)+\bar{\nu}_{\mu}\left(\nu_{\mu}\right) .
\end{aligned}
$$

The ratio of muon neutrino flux to electron neutrino flux, $\phi_{\nu_{\mu}} / \phi_{\nu_{e}}$, was predicted to be 2 .

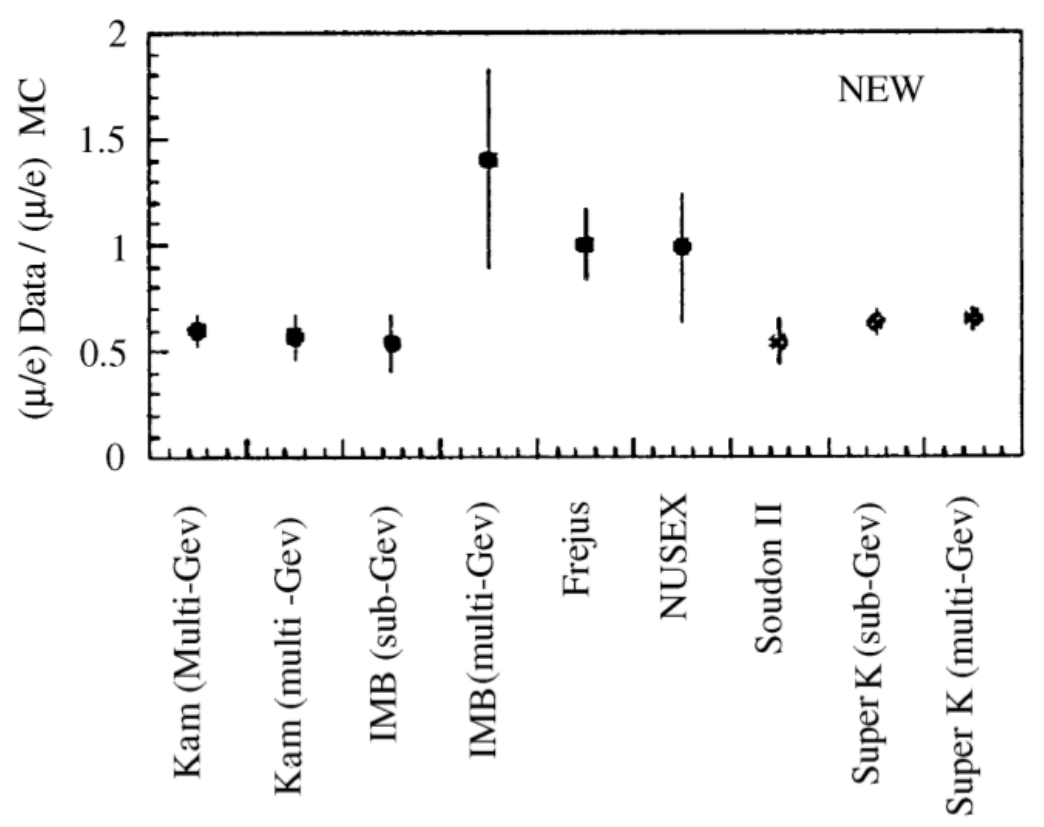

Figure 1.5: Atmospheric neutrino deficit observed in a variety of experiments. The y axis is the ratio of measured $\phi_{\nu_{\mu}} / \phi_{\nu_{e}}$ rates divided by the prediction from Monte Carlo simulation. Figure taken from [37]. 
However, a number of experiments [38-43] confirmed that this ratio was smaller than what we expected. The ratios of measured $\phi_{\nu_{\mu}} / \phi_{\nu_{e}}$ divided by the Monte Carlo prediction from several experiments are summarized in Figure 1.5. The double ratio is smaller than 1 ( $\sim 0.6$ yielded by statistically linear fit), indicates a shortfall of $\nu_{\mu}\left(\bar{\nu}_{\mu}\right)$ component in total neutrino flux at observed locations on the Earth in comparison with $\nu_{\mu}\left(\bar{\nu}_{\mu}\right)$ component on the Sun.

\subsubsection{Neutrino oscillation theory}

The solar and atmospheric neutrino anomalies present compelling examples of neutrino oscillations, which spring from a quantum mechanical mixing between the mass and flavor eigenstates of neutrinos. Neutrinos, which are observed in the experiments, are created with other fermions through weak nuclear force, which does not change the flavor of the particle. The $\nu_{e}, \nu_{\mu}$ and $\nu_{\tau}$ are labeled as the flavor eigenstates of neutrinos (generalized as $\nu_{\alpha}$ ). The only way flavor eigenstates of neutrinos can be constructed to be invariant under weak nuclear force is a mixture of exactly the right portion of the three flavor-mixed eigenstates $\nu_{1}, \nu_{2}$ and $\nu_{3}$. Since each of these eigenstates should have a definite mass, it is labeled as the mass eigenstate, $\nu_{i}$. For a neutrino of flavor $\alpha$ at its production point $(\mathrm{t}=0)$, its state can be simply expressed as follows:

$$
\left|\nu_{\alpha}(0)\right\rangle=U_{\alpha i}\left|\nu_{i}(0)\right\rangle \text { where } \alpha=e, \mu, \tau \text { and } i=1,2,3 .
$$


Here $U_{\alpha i}$ is the leptonic mixing matrix, or named the $\mathrm{PMNS}^{10}$ matrix $[12,44$, 45]. For neutrinos propagating freely in the vacuum, their mass eigenstates $\left|\nu_{i}\right\rangle$ evolve as a free particles:

$$
\left|\nu_{i}(t)\right\rangle=e^{-i \vec{p} \cdot \vec{x}}\left|\nu_{i}(0)\right\rangle
$$

where $\vec{p}$ is the four-dimensional momentum and $\vec{x}$ is the four-dimensional coordinate of neutrino at time $t$. Thus the flavor eigenstate $\left|\nu_{\alpha}(\vec{t})\right\rangle$ propagates as follows:

$$
\left|\nu_{\alpha}(t)\right\rangle=\sum_{i=1}^{3} U_{\alpha i} e^{-i \overrightarrow{p_{i}} \cdot \vec{x}}\left|\nu_{i}(0)\right\rangle .
$$

Assuming that the neutrino mass $m_{i}$ is much smaller than its energy $E_{i}$, we have following approximation:

$$
E_{i}=\sqrt{p_{i}^{2}+m_{i}^{2}} \approx p_{i}+\frac{m_{i}^{2}}{2 p_{i}} \approx E+\frac{m_{i}^{2}}{2 E},
$$

where $E$ is the average of all $E_{i}$. For relativistic neutrinos, $t \approx L$ and $\sum_{i=k}^{3} p_{k} x_{k} \approx E_{i} L$ ( $\mathrm{L}$ is the neutrino propagation distance):

$$
\overrightarrow{p_{i}} \cdot \vec{x}=E_{i} \cdot t-\sum_{i=k}^{3} p_{k} x_{k} \approx\left(\frac{m_{i}^{2} L}{2 E}\right) .
$$

Substitute Eq. (1.14) into Eq. (1.13) yields:

$$
\left|\nu_{\alpha}(t)\right\rangle=\sum_{i=1}^{3} U_{\alpha i} e^{-i \frac{m_{i}^{2} L}{2 E}} .
$$

\footnotetext{
${ }^{10}$ Abbreviated after Pontecorvo, Maki, Nakagawa, and Sakata.
} 
The probability of observing a neutrino of flavor $\beta\left(\nu_{\beta}\right)$ at time $t$ (equivalent to distance $L)$ from a neutrino of original flavor $\alpha\left(\nu_{\alpha}\right)$ is then given by:

$$
\begin{aligned}
P_{\nu_{\alpha} \rightarrow \nu_{\beta}}(t)= & \left|\left\langle\nu_{\beta} \mid \nu_{\alpha}(t)\right\rangle\right|^{2} \\
= & \delta_{\alpha \beta}-4 \sum_{i>j} \Re\left(U_{\alpha i}^{*} U_{\beta i} U_{\alpha j} U_{\beta j}^{*}\right) \sin ^{2}\left(\frac{\Delta m_{i j}^{2} L}{4 E}\right) \\
& +2 \sum_{i>j} \Im\left(U_{\alpha i}^{*} U_{\beta i} U_{\alpha j} U_{\beta j}^{*}\right) \sin \left(\frac{\Delta m_{i j}^{2} L}{4 E}\right),
\end{aligned}
$$

where $\Delta m_{i j}^{2}=m_{i}^{2}-m_{j}^{2}$ and we can rewrite:

$$
\frac{\Delta m_{i j}^{2} L}{4 E} \approx 1.267 \frac{\Delta m_{i j}^{2}\left[e V^{2}\right] \times L[k m]}{E[\mathrm{GeV}]} .
$$

The neutrino oscillation probabilities depend on the elements of the PMNS matrix, which can be generally formulated as follows:

$$
U_{\mathrm{PMNS}}=\left(\begin{array}{ccc}
U_{e 1} & U_{e 2} & U_{e 3} \\
U_{\mu 1} & U_{\mu 2} & U_{\mu 3} \\
U_{\tau 1} & U_{\tau 2} & U_{\tau 3}
\end{array}\right) .
$$

This matrix is unitary and normally represented by three mixing angles $\left(\theta_{12}, \theta_{23}\right.$, and $\left.\theta_{13}\right)$, one Dirac CP phase $\left(\delta_{\mathrm{CP}}\right)$ and two Majorana phases $\left(\alpha_{1}\right.$ and $\left.\alpha_{2}\right)$ :

$$
\begin{aligned}
U_{\mathrm{PMNS}}= & \left(\begin{array}{ccc}
c_{12} c_{13} & s_{12} c_{13} & s_{13} e^{-i \delta} \\
-s_{12} c_{23}-c_{12} s_{23} s_{13} e^{i \delta_{\mathrm{CP}}} & c_{12} c_{23}-s_{12} s_{23} s_{13} e^{i \delta_{\mathrm{CP}}} & s_{23} c_{13} \\
s_{12} s_{23}-c_{12} c_{23} s_{13} e^{i \delta_{\mathrm{CP}}} & -c_{12} s_{23}-s_{12} c_{23} s_{13} e^{i \delta_{\mathrm{CP}}} & c_{23} c_{13}
\end{array}\right) \\
& \times\left(\begin{array}{ccc}
e^{i \alpha_{1} / 2} & 0 & 0 \\
0 & e^{i \alpha_{2} / 2} & 0 \\
0 & 0 & 1
\end{array}\right)
\end{aligned}
$$

where $s_{i j}=\sin \theta_{i j}$ and $c_{i j}=\cos \theta_{i j}$.

Besides three mixing angles and CP phases, the oscillation probabilities 
(as shown in Eq. (1.16)), in principle, are driven by the three mass-squared splittings $\Delta m_{i j}^{2}$. The experimental evidence shows two scales of mass-squared splitting. While the smaller of these two, named the solar mass-squared splitting $\Delta m_{12}^{2}$, is around $7.5 \times 10^{-5} \mathrm{eV}^{2}$, the larger of these two, named the atmospheric mass-squared splitting $\left|\Delta m_{32}^{2}\right|$, is around $2.4 \times 10^{-3} \mathrm{eV}^{2}[9]$.

Most experiments would be sensitive to one out of these two scales, i.e $\Delta m_{a}^{2} L / E=\mathcal{O}(1)$ while $\Delta m_{b}^{2} L / E \ll \mathcal{O}(1)$ or $\Delta m_{b}^{2} L / E \gg \mathcal{O}(1)$. In this case, the oscillation probabilities are approximately rewritten as follows:

$$
\begin{aligned}
& P_{\vec{\nu}_{\alpha} \rightarrow \grave{\nu}_{\beta \neq \alpha}} \approx 4\left|\sum_{i \mathrm{Up}} U_{\alpha i}^{*} U_{\beta i}\right|^{2} \sin ^{2}\left(1.267 \frac{\Delta m_{a}^{2} L}{E}\right), \\
& P_{\vec{\nu}_{\alpha} \rightarrow \vec{\nu}_{\alpha}^{\prime}} \approx 1-4 \sum_{i \mathrm{Up}}\left|U_{\alpha}\right|^{2}\left(1-\sum_{i \mathrm{Up}}\left|U_{\alpha}\right|^{2}\right) \sin ^{2}\left(1.267 \frac{\Delta m_{a}^{2} L}{E}\right),
\end{aligned}
$$

where " $i$ Up" denotes a sum over only those neutrino mass eigenstates that lie above $\Delta m_{a}^{2}$ or, alternatively, only those that lie below it. The following highlights some examples from the baseline neutrino experiments.

For $\frac{\Delta m_{21}^{2} L}{E} \ll 1$, there is no contribution from the solar term. With $\Delta m_{31}^{2} \approx \Delta m_{32}^{2}$ assumption, the oscillation probabilities for a number of channels are simplified as follows: 
- Electron neutrino disappearance: The Daya Bay, RENO ${ }^{11}$ and Double Chooz experiments $[17,46,47]$ are sensitive to this channel.

$$
\begin{aligned}
P_{\nu_{e} \rightarrow \nu_{e}} & \approx 1-4\left|U_{e 3}\right|^{2}\left(1-\left|U_{e 3}\right|^{2}\right) \sin ^{2}\left(1.267 \frac{\Delta m_{32}^{2} L}{E}\right) \\
& \approx 1-\sin ^{2} 2 \theta_{13} \sin ^{2}\left(1.267 \frac{\Delta m_{32}^{2} L}{E}\right) .
\end{aligned}
$$

- Muon neutrino disappearance: The MINOS, T2K, and incoming $\mathrm{NO} \nu \mathrm{A}^{12}$ experiments [48-50] are sensitive to this channel.

$$
\begin{aligned}
P_{\nu_{\mu} \rightarrow \nu_{\mu}} & \approx 1-4\left|U_{\mu 3}\right|^{2}\left(1-\left|U_{\mu 3}\right|^{2}\right) \sin ^{2}\left(1.267 \frac{\Delta m_{32}^{2} L}{E}\right) \\
& \approx 1-\cos ^{2} \theta_{13} \sin ^{2} 2 \theta_{23} \sin ^{2}\left(1.267 \frac{\Delta m_{32}^{2} L}{E}\right)
\end{aligned}
$$

- Electron neutrino appearance: The MINOS, T2K and incoming $\mathrm{NO} \nu \mathrm{A}$ experiments $[18,50,51]$ are sensitive to this channel.

$$
\begin{aligned}
P_{\nu_{\mu} \rightarrow \nu_{e}} & \approx 4\left|U_{e 3}\right|^{2}\left|U_{\mu 3}\right|^{2} \sin ^{2}\left(1.267 \frac{\Delta m_{32}^{2} L}{E}\right) \\
& \approx \sin ^{2} 2 \theta_{13} \sin ^{2} \theta_{23} \sin ^{2}\left(1.267 \frac{\Delta m_{32}^{2} L}{E}\right)
\end{aligned}
$$

For $\frac{\Delta m_{32}^{2} L}{E} \gg 1$, the oscillation pattern driven by the atmospheric term vanishes due to the comparatively low reconstructed energy resolution, and there is only a contribution from the solar term. The probability of $\nu_{e}$ disappearance, which is studied by the Kamioka Liquid Scintillator Antineutrino

\footnotetext{
${ }^{11}$ RENO abbreviated for Reactor Experiment for Neutrino Oscillations.

${ }^{12} \mathrm{NO} \nu \mathrm{A}$ abbreviated for NuMI Off-Axis $\nu_{e}$ Appearance.
} 
Detector (KamLAND) experiment [16], has the following expression:

$$
\begin{aligned}
P_{\nu_{e} \rightarrow \nu_{e}} & \approx 1-4\left|U_{e 1}\right|^{2}\left(1-\left|U_{e 1}\right|^{2}\right) \sin ^{2}\left(1.267 \frac{\Delta m_{21}^{2} L}{E}\right) \\
& \approx 1-4\left|U_{e 1}\right|^{2}\left|U_{e 2}\right|^{2} \sin ^{2}\left(1.267 \frac{\Delta m_{21}^{2} L}{E}\right) \\
& \approx\left|U_{e 1}\right|^{4}+\left|U_{e 2}\right|^{2}+2\left|U_{e 1}\right|^{2}\left|U_{e 2}\right|^{2} \cos \left(2.534 \frac{\Delta m_{21}^{2} L}{E}\right) \\
& \approx 1-\cos ^{4} \theta_{13} \sin ^{2} 2 \theta_{12} \cos \left(2.534 \frac{\Delta m_{21}^{2} L}{E}\right)
\end{aligned}
$$

Here we did use the fact that $\left|U_{e 1}\right|^{2}+\left|U_{e 2}\right|^{2}+\left|U_{e 3}\right|^{2}=1$ and $\left|U_{e 3}\right|^{2} \ll$ $\left|U_{e 1}\right|^{2},\left|U_{e 2}\right|^{2}$.

Each neutrino experiment is normally sensitive to a specific set of oscillation parameters and cannot handle conclusively the degeneracies due to the correlation between the CP phase and $\theta_{13}$ mixing angle, the sign of $\Delta m_{32}^{2}$, and the octant of mixing angle $\theta_{23}$. These degeneracies and correlations are discussed in Appendix A. To complete the picture of neutrino oscillation, it is necessary to have a global framework which combines data sets in different channels from a variety of neutrino experiments.

\subsubsection{Matter effect in neutrino oscillations}

The neutrino oscillation described in the previous section is only valid in a vacuum. When propagating through matter, coherence forward scattering between electron neutrinos and electrons results in extra interaction potential energy, given by:

$$
V_{\text {mat }}= \begin{cases}+\sqrt{2} G_{F} N_{e} & \text { for } \nu_{e} \\ -\sqrt{2} G_{F} N_{e} & \text { for } \bar{\nu}_{e}\end{cases}
$$


where $G_{F}$ is the Fermi weak coupling constant and $N_{e}$ is the electron density. As a result, this potential distorts the neutrino oscillation probability. The matter effect in neutrino oscillations is knowns as the Mikheyev-SmirnovWolfenstein (MSW) effect $[52,53]$. To demonstrate the impact of matter effect on the neutrino oscillation probabilities, we consider a simple two-flavor oscillation between $\nu_{e}$ and $\nu_{\alpha}(\alpha=e, \mu, \tau)$. Their mixing of two mass eigenstate $\nu_{1}$ and $\nu_{2}$ at $t=0$ can be expressed as follows:

$$
\left(\begin{array}{l}
\left|\nu_{e}(0)\right\rangle \\
\left|\nu_{\alpha}(0)\right\rangle
\end{array}\right)=\left(\begin{array}{cc}
\cos \theta & \sin \theta \\
-\sin \theta & \cos \theta
\end{array}\right)\left(\begin{array}{l}
\left|\nu_{1}(0)\right\rangle \\
\left|\nu_{2}(0)\right\rangle
\end{array}\right)
$$

In the vacuum, the time evolution of mass eigenstates is governed by:

$$
\frac{\partial}{\partial t}\left(\begin{array}{l}
\left|\nu_{1}(t)\right\rangle \\
\left|\nu_{2}(t)\right\rangle
\end{array}\right)_{\text {vac }}=H_{0}\left(\begin{array}{l}
\left|\nu_{1}(0)\right\rangle \\
\left|\nu_{2}(0)\right\rangle
\end{array}\right)
$$

where the Hamiltonian of the mass eigenstates is given by:

$$
\begin{aligned}
H_{0} & =\left(\begin{array}{cc}
E_{1} & 0 \\
0 & E_{2}
\end{array}\right) \approx\left(\begin{array}{cc}
p+\frac{m_{1}^{2}}{2 E} & 0 \\
0 & p+\frac{m_{2}^{2}}{2 E}
\end{array}\right) \\
& =\left(p+\frac{m_{1}^{2}}{2 E}\right) \mathbb{1}+\left(\begin{array}{cc}
0 & 0 \\
0 & \frac{\Delta m^{2}}{2 E}
\end{array}\right) .
\end{aligned}
$$

Here $E, p$ are the neutrino energy and the neutrino momentum respectively; $m_{1}, m_{2}$ are the masses of two eigenstates $\nu_{1}$ and $\nu_{2} ; \Delta m^{2}=m_{2}^{2}-m_{1}^{2}$. Since any term in the Hamiltonian proportional to the identity matrix gives no observable consequences, we can ignore $\left(p+\frac{m_{1}^{2}}{2 E}\right) \mathbb{1}$ term in the above expression. By taking the unitary conjugation of Eq. (1.27) and substituting to Eq. (1.28), we obtain:

$$
\frac{\partial}{\partial t}\left(\begin{array}{l}
\left|\nu_{e}(t)\right\rangle \\
\left|\nu_{\alpha}(t)\right\rangle
\end{array}\right)_{\text {vac }}=\frac{\Delta m^{2}}{2 E}\left(\begin{array}{cc}
\sin ^{2} \theta & \frac{1}{2} \sin 2 \theta \\
\frac{1}{2} \sin 2 \theta & \cos ^{2} \theta
\end{array}\right)\left(\begin{array}{l}
\left|\nu_{e}(0)\right\rangle \\
\left|\nu_{\alpha}(0)\right\rangle
\end{array}\right) .
$$


By taking into account of the extra interaction potential energy, this equation is modified by:

$$
\begin{aligned}
\frac{\partial}{\partial t}\left(\begin{array}{l}
\left|\nu_{e}(t)\right\rangle \\
\left|\nu_{\alpha}(t)\right\rangle
\end{array}\right)_{\text {mat }} & =H_{\text {mat }}\left(\begin{array}{l}
\left|\nu_{e}(0)\right\rangle \\
\left|\nu_{\alpha}(0)\right\rangle
\end{array}\right) \\
& =\frac{\Delta m^{2}}{2 E}\left(\begin{array}{cc}
\sin ^{2} \theta+\frac{2 E}{\Delta m^{2}} V_{\text {mat }} & \frac{1}{2} \sin 2 \theta \\
\frac{1}{2} \sin 2 \theta & \cos ^{2} \theta
\end{array}\right)\left(\begin{array}{l}
\left|\nu_{e}(0)\right\rangle \\
\left|\nu_{\alpha}(0)\right\rangle
\end{array}\right)
\end{aligned}
$$

The eigenvalues of modified Hamiltonian in Eq. (1.30) are:

$$
\begin{aligned}
& \lambda_{1}=\frac{1}{2}\left(V_{\text {mat }}+\frac{\Delta m^{2}}{2 E}\left(1-\sqrt{\sin ^{2} 2 \theta+\left(\cos 2 \theta-\frac{2 E}{\Delta m^{2}} V_{\text {mat }}\right)^{2}}\right)\right) \\
& \lambda_{2}=\frac{1}{2}\left(V_{\text {mat }}+\frac{\Delta m^{2}}{2 E}\left(1+\sqrt{\sin ^{2} 2 \theta+\left(\cos 2 \theta-\frac{2 E}{\Delta m^{2}} V_{\text {mat }}\right)^{2}}\right)\right) .
\end{aligned}
$$

This yields an effective mass-squared splitting by:

$$
\Delta m_{m}^{2}=\lambda_{2}-\lambda_{1}=\frac{\Delta m^{2}}{2 E} \sqrt{\sin ^{2} 2 \theta+\left(\cos 2 \theta-\frac{2 E}{\Delta m^{2}} V_{\text {mat }}\right)^{2}} .
$$

We can find the corresponding mixing angle $\theta_{m}$ in term of $\theta$ and $V_{\text {mat }}$ by considering:

$$
H_{\text {mat }}=\left(\begin{array}{cc}
\cos \theta_{m} & \sin \theta_{m} \\
-\sin \theta_{m} & \cos \theta_{m}
\end{array}\right)\left(\begin{array}{cc}
0 & 0 \\
0 & \frac{\Delta m_{m}^{2}}{2 E}
\end{array}\right)\left(\begin{array}{cc}
\cos \theta_{m} & \sin \theta_{m} \\
-\sin \theta_{m} & \cos \theta_{m}
\end{array}\right) .
$$

Matching Hamiltonians in Eq. (1.33) and Eq. )1.30) yields:

$$
\sin 2 \theta_{m}=\frac{\sin 2 \theta}{\sqrt{\sin ^{2} 2 \theta+\left(\cos 2 \theta-\frac{2 E}{\Delta m^{2}} V_{\text {mat }}\right)^{2}}}
$$

This exercise demonstrates that the matter effect modifies both the mixing angle (expressed in Eq. (1.34)) and the mass-squared splitting (expressed in Eq. (1.32)). Also, the amplitude of matter effect depends on the neutrino 
energy and the matter density. A resonance occurs at $V_{\text {mat }} / E=2 / \Delta m^{2} \cos 2 \theta$ and results in maximum mixing $\sin ^{2} \theta_{m}=1$. Figure 1.6 shows the atmospheric resonance $(L>800 \mathrm{~km}$ at $E \sim 1 \mathrm{GeV})$ in the $\nu_{\mu} \rightarrow \nu_{e}$ transition with the normal hierarchy but not inverted hierarchy. This indicates that matter effect is a powerful tool for solving the neutrino mass hierarchy.
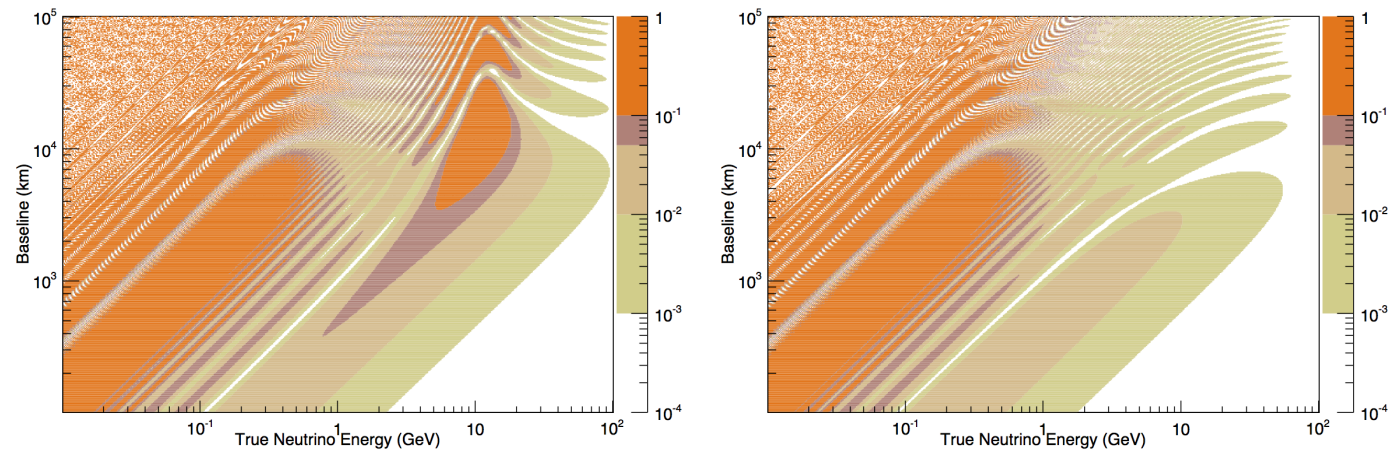

Figure 1.6: $\nu_{\mu} \rightarrow \nu_{e}$ oscillation probabilities in matter with baseline of $1250 \mathrm{~km}$ plotted for the normal hierarchy (left) and the inverted hierarchy (right).
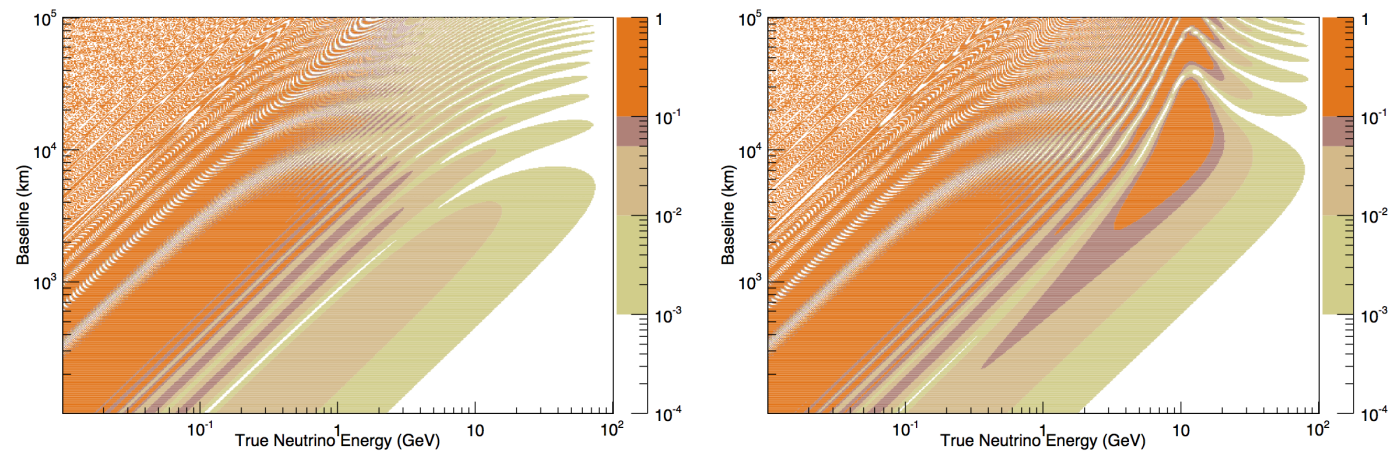

Figure 1.7: $\bar{\nu}_{\mu} \rightarrow \bar{\nu}_{e}$ oscillation probabilities in matter with baseline of $1250 \mathrm{~km}$ plotted for normal hierarchy (left) and the inverted hierarchy (right). 
In addition, the matter treats neutrino and antineutrino oscillation in different directions (see Eq. (1.26)). Figure 1.7 shows the atmospheric resonance $(L>800 \mathrm{~km}$ at $E \sim 1 \mathrm{GeV})$ in the $\bar{\nu}_{\mu} \rightarrow \bar{\nu}_{e}$ transition with the inverted hierarchy but not normal hierarchy. Thus, taking advantage of matter effect in both $\nu_{\mu} \rightarrow \nu_{e}$ and $\bar{\nu}_{\mu} \rightarrow \bar{\nu}_{e}$ would be very helpful for resolving the neutrino mass hierarchy and measuring the $\mathrm{CP}$-violation phase.

\subsubsection{Antineutrino and neutrino oscillations}

Under the charge-parity (CP) symmetry, we expect that [54]:

$$
P_{\bar{\nu}_{\alpha} \rightarrow \bar{\nu}_{\beta}}=P_{\nu_{\alpha} \rightarrow \nu_{\beta}}\left(\delta_{\mathrm{CP}} \rightarrow-\delta_{\mathrm{CP}}, V_{\mathrm{mat}} \rightarrow-V_{\mathrm{mat}}\right)
$$

where $V_{\text {mat }}$ is the effective potential caused by the effect of matter in which neutrinos pass through (discussed in Section 1.4.4). Even in a vacuum (i.e., $V \equiv 0$ ), the neutrino oscillation probability and its CP process, in general, are not the same if $\delta_{C P} \neq 0$. However, in the disappearance mode (i.e., $\alpha \equiv \beta$ ) the CP phase is not involved. Also, in this mode, the time reversal operator does not change the physical situation, $\mathcal{T}\left(\nu_{\alpha} \rightarrow \nu_{\alpha}\right)=\left(\nu_{\alpha} \rightarrow \nu_{\alpha}\right)$. Thus, under

the CPT symmetry, the rate of $\nu_{\alpha}$ and $\bar{\nu}_{\alpha}$ appearances would be identical in the vacuum.

In this dissertation, the $\bar{\nu}_{\mu}$ and $\nu_{\mu}$ disappearance data is described by an effective two-flavor mode with a single mass-squared $\Delta m^{2}$ splitting and mixing angle $\theta$. In this approximation, the $\bar{\nu}_{\mu}$ and $\nu_{\mu}$ survival probabilities are 
given by:

$$
P\left(\bar{\nu}_{\mu} \rightarrow \bar{\nu}_{\mu}\right)=P\left(\nu_{\mu} \rightarrow \nu_{\mu}\right)=1-\sin ^{2} 2 \theta \sin ^{2}\left(1.267 \frac{\Delta m^{2} L[k m]}{E[G e V]}\right)
$$

where $L$ and $E$ are the neutrino propagation distance and the neutrino energy respectively. This approximation is derived from the three-flavor neutrino oscillation model, which is governed by two mass-squared splitting $\Delta m_{21}^{2}$ and $\Delta m_{32}^{2}$, three mixing angle $\theta_{12}, \theta_{23}$ and $\theta_{13}$, and one single CP phase $\delta_{\mathrm{CP}}$. The effective parameters in this two-flavor model are given by [55]:

$$
\begin{aligned}
& \Delta m^{2}=\Delta m_{32}^{2}+\sin _{12}^{2} \Delta m_{21}^{2}+\cos \delta_{\mathrm{CP}} \sin \theta_{13} \sin 2 \theta_{12} \tan \theta_{23} \Delta m_{21}^{2}, \\
& \sin ^{2} 2 \theta=4 \sin ^{2} \theta_{23} \cos ^{2} \theta_{13}\left(1-\sin \theta_{23}^{2} \cos \theta_{13}^{2}\right) .
\end{aligned}
$$

Evidently, $\Delta m^{2} \simeq \Delta m_{32}^{2}$ and $\sin ^{2} 2 \theta \simeq \sin ^{2} \theta_{23}$ when considering that $\Delta m_{21}^{2} \ll$ $\Delta m_{32}^{2}$ and $\sin ^{2} \theta_{13} \simeq 0$.

To account for the matter effect on the neutrino propagation, the mixing angle $\theta_{13}$ is replaced by $\theta_{13}^{\mathrm{m}}$ (described in Section 1.4.4), given by:

$$
\sin 2 \theta_{13}^{\mathrm{m}}=\frac{\sin 2 \theta_{13}}{\sin ^{2} 2 \theta_{13}+\left(A-\cos 2 \theta_{13}\right)^{2}},
$$

where $A \equiv \pm 2 \sqrt{2} G_{F} n_{e} E_{\nu} /\left(\Delta m_{32}^{2}+\Delta m_{21}^{2}\right), G_{F}$ is the Fermi weak coupling constant, $E_{\nu}$ is the neutrino energy, $n_{e}$ is the electron density and the sign of $\mathrm{A}$ is positive (negative) for neutrinos (antineutrinos). Moreover, to have exact formulae for $\bar{\nu}_{\mu}$ and $\nu_{\mu}$ survival probabilities, we need to add the sub-leading term, given by:

$$
P^{\text {sub-term }}\left(\nu_{\mu} \rightarrow \nu_{\mu}\right)=-4\left|U_{\mu 2}\right|^{2}\left|U_{\mu 1}\right|^{2} \sin ^{2}\left(1.267 \frac{\Delta m_{21}^{2} L(k m)}{E(G e V)}\right)
$$


where $U_{\mu 1}$ and $U_{\mu 2}$ are two elements of the PMNS matrix, which is defined in Eq. (1.18). Figure 1.8a shows the small difference between the approximate and the exact $\nu_{\mu}$ survival probabilities. At $3 \mathrm{GeV}$ (peak of $\bar{\nu}$ energy in $\bar{\nu}_{\mu}$ beam in MINOS), this difference is around $2 \%$.

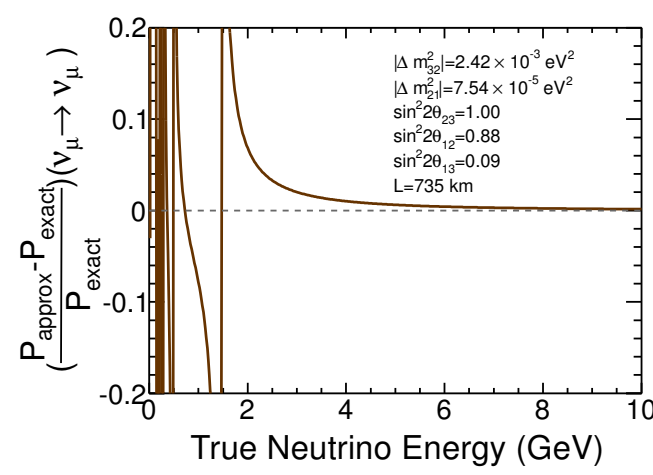

(a) Exact vs. approximate solutions

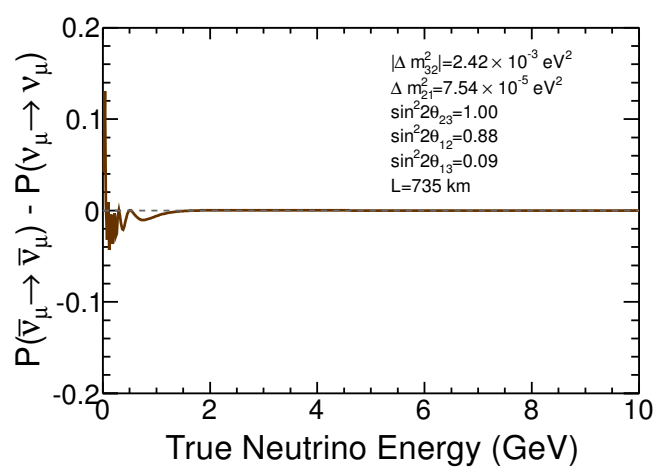

(b) $\bar{\nu}_{\mu}$ vs. $\nu_{\mu}$

Figure 1.8: The difference between the exact and approximate $\nu_{\mu}$ survival probabilities (left) and the difference between the exact $\bar{\nu}_{\mu}$ and $\nu_{\mu}$ survival probabilities (right).

In a vacuum, the $\bar{\nu}_{\mu}$ and $\nu_{\mu}$ survival probabilities are identical. Figure $1.8 \mathrm{~b}$ shows the impact of matter effect on their difference. The difference is very small in comparison to the uncertainties of the measurement of oscillation parameters. Thus, the effective two-flavor model is good approximation for measuring both $\bar{\nu}_{\mu}$ and $\nu_{\mu}$ disappearances in MINOS.

\subsubsection{Alternative disappearance models}

Along with neutrino oscillation, a number of theoretical models have been proposed to explain the observed neutrino deficits, either in whole or in 
part. In the context of this dissertation, neutrino decay, neutrino decoherence and large-extra dimensions are introduced. Although the theoretical motivations for neutrino decay and neutrino decoherence are very different, they are both characterized by the same phenomenological signature: an exponential damping of the flavor conversion probabilities. The large-extra dimensionsbased model, on other hand, assumes the existence of right-handed neutrinos propagating in more than four dimensional space and has a subdominant effect on top of the standard oscillations.

\section{Neutrino decay}

Neutrino decay was proposed as the alternative interpretation for solar and atmospheric neutrino anomalies [56]. The main idea is based on the hypothetical instability of the mass eigenstates. These neutrino eigenstates can be decay into either "detectable" neutrinos or "invisible" neutrinos. Due to the neutrino mixing, the probability of decay is basically the incoherent sum of decay modes into all the neutrino mass eigenstates. Since the neutrino has mass, the heavier one might decay into the lighter ones with lifetime of $\tau_{0}$. To

derive the formulae of survival probability, the phase $e^{-\frac{\tau}{2 \tau_{0}}}$ is added into the free-wave expression Eq. (1.12). In the effective two-flavor mode, this finally yields the following oscillation probability for $\nu_{\mu}$ survival after propagating a 
distance $L$ as follows:

$$
\begin{aligned}
P_{\nu_{\mu} \rightarrow \nu_{\mu}}(L)= & \sin ^{4} \theta_{23}+\cos ^{4} \theta_{23} \exp \left(-\frac{m_{2} L}{E_{\nu} \tau_{0}}\right) \\
& +2 \sin ^{2} \theta_{23} \cos ^{2} \theta_{23} \exp \left(-\frac{m_{2} L}{2 . E_{\nu} \tau_{0}}\right) \cos \left(\frac{\Delta m_{32}^{2} L}{2 E_{\nu}}\right),
\end{aligned}
$$

where $E_{\nu}$ is the neutrino energy; $m_{2}$ is the mass of $\nu_{2}$ mass eigenstate. In the limitation of stable neutrinos $\tau_{0} \rightarrow \infty$, reduces to the oscillation formulae. Additionally, when $\Delta m_{32}^{2}$ is very small comparatively to the $E_{\nu} / L$ ratio, the $\nu_{\mu}$ survival probability can be approximated by:

$$
P_{\nu_{\mu} \rightarrow \nu_{\mu}}(L)=\left(\sin ^{2} \theta_{23}+\cos ^{2} \theta_{23} \exp \left(-\frac{m_{2} L}{2 \cdot E_{\nu} \tau_{0}}\right)\right)^{2} .
$$

\section{Neutrino decoherence}

The possible decoherence effect in atmospheric neutrino oscillation was proposed in [57]. In this model, the disappearance of one flavor neutrino happens due to a foamy or fuzzy space-time background, which makes path lengths indeterminate and gradually destroys the phase relationship among the mass eigenstates. General arguments lead to the following formula of $\nu_{\mu}$ survival probability after propagating a distance $L$ in the effective two-flavor model:

$$
P_{\nu_{\mu} \rightarrow \nu_{\mu}}(L)=1-\frac{1}{2} \sin ^{2} 2 \theta_{23}\left(1-\exp \left(-\frac{\mu^{2} L}{2 E_{\nu}}\right) \cos \left(\frac{\Delta m_{32}^{2} L}{2 E_{\nu}}\right)\right)
$$

where the parameter $\mu^{2}$ determines the amplitude of the decoherence. There are two limits in Eq. (1.41): 
- Pure oscillation, when $\mu \equiv 0$ :

$$
P_{\nu_{\mu} \rightarrow \nu_{\mu}}(L)=1-\sin ^{2} 2 \theta_{23} \sin ^{2}\left(\frac{\Delta m_{32}^{2} L}{4 E_{\nu}}\right) .
$$

- Pure decoherence, when $\Delta m_{32}^{2} \equiv 0$ :

$$
P_{\nu_{\mu} \rightarrow \nu_{\mu}}(L)=1-\frac{1}{2} \sin ^{2} 2 \theta_{23}\left(1-\exp \left(-\frac{\mu^{2} L}{2 E_{\nu}}\right)\right) .
$$

Figure 1.9 shows the $\nu_{\mu}$ survival probability with different models. In comparison to neutrino decay and neutrino decoherence, neutrino oscillation yields sharper deficit dip. The MINOS data [58] disfavor neutrino decay and neutrino decoherence at $7.8 \sigma$ and $9.7 \sigma$ respectively.

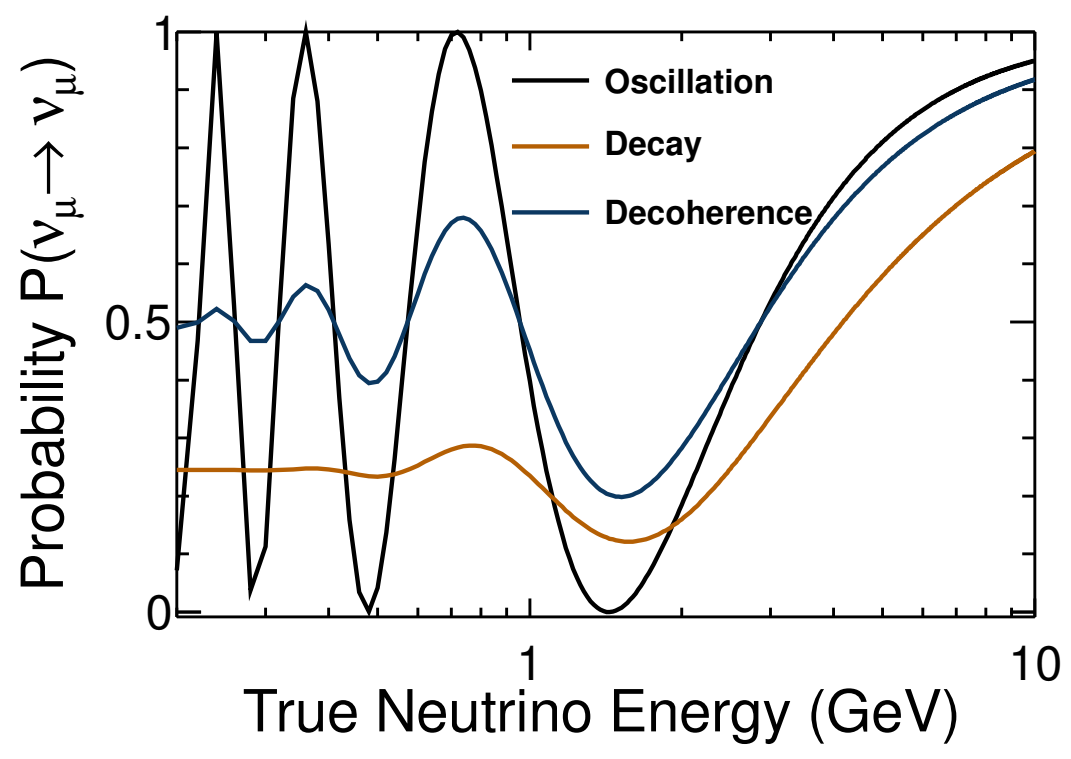

Figure 1.9: Comparison of $\nu_{\mu}$ survival probabilities from three models: oscillation (black), decay (orange) and decoherence (blue). The plot is made with $\Delta m_{23}^{2}=2.4 \times 10^{-3} \mathrm{eV}^{2}, \theta_{23}=0.78,735 \mathrm{~km}$ of baseline, $m_{2} / \tau_{0}=2 \times 10^{-3} \mathrm{eV} / \mathrm{s}$ for decay and $\mu=2 \times 10^{-2}$ for decoherence. 


\section{Large extra dimensions}

Neutrino oscillation formalism with the large-extra dimensions (LED) is described in [59]. Right-handed neutrinos are hypothetically propagated in a more-than four-dimensional space. These neutrinos are referred as the KazulaKlein (KK) neutrino modes. The Yukawa coupling of the KK neutrino modes with the three standard left-handed neutrinos and the Higgs field leads to a Dirac mass term of neutrinos. In general, mixing between the KK neutrino modes introduces an additional mixing matrix, $W$, along with the standard PMNS matrix in Eq. (1.11). The transition probability of $\nu_{\alpha}$ into $\nu_{\beta}$ can be given by:

$$
\begin{aligned}
& P_{\nu_{\alpha} \rightarrow \nu_{\beta}}(L)=\left|A_{\nu_{\alpha} \rightarrow \nu_{\beta}}(L)\right|^{2}, \\
& A_{\nu_{\alpha} \rightarrow \nu_{\beta}}(L)=\sum_{i, j, k=1}^{3} \sum_{N=0}^{\infty} U_{\alpha i} U_{\beta k}^{*} W_{i j}^{(0 N) *} W_{k j}^{(0 N)} \times \exp \left(i \frac{\lambda_{j}^{(N) 2} L}{2 E a^{2}}\right),
\end{aligned}
$$

where $E$ is the neutrino energy; $L$ is the neutrino propagation distance; $a$ is the size of extra dimension; and $\lambda_{j}^{(N)}$ is the eigenvalues of Hamiltonian [59]. Figure 1.10 shows the perturbation of $\nu_{\mu}$ survival probability caused by the effect of LED with a baseline of $735 \mathrm{~km}$ (specific to the MINOS experiment). A study [59] shows that the neutrino oscillation experiments can be sensitive to a LED search down to $1 \mu m$. 


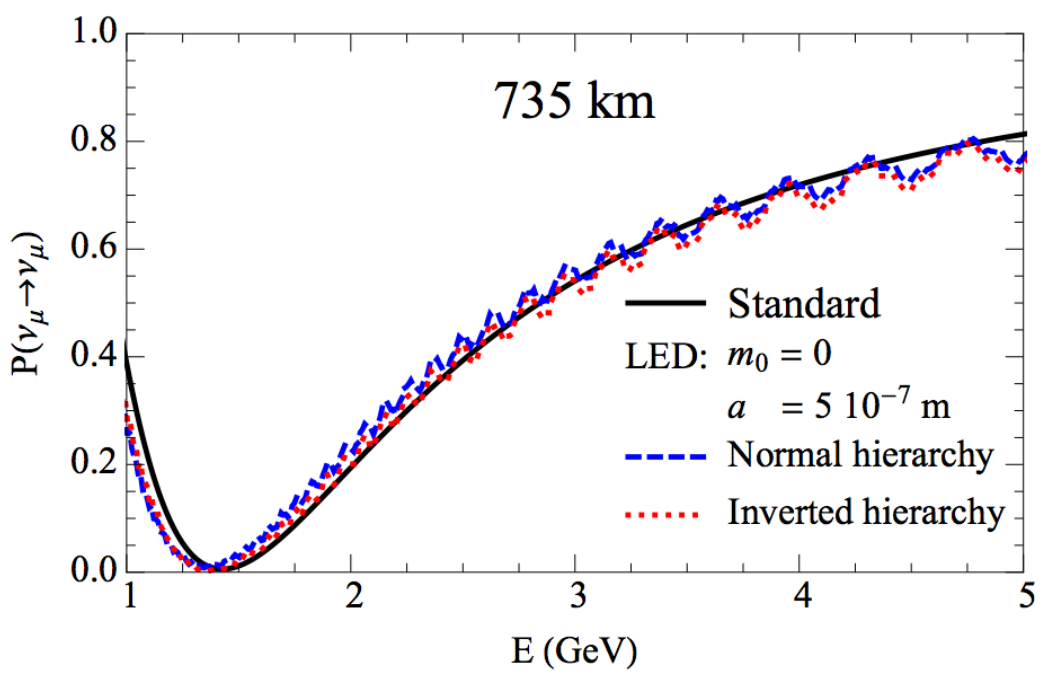

Figure 1.10: The $\nu_{\mu}$ survival probability with standard formalism (black) and with LED effect (blue for normal mass hierarchy and red for inverted mass hierarchy.) The lightest mass of neutrinos is assumed to be zero. Figure taken from [59].

In addition to neutrino decay and neutrino decoherence, a number of models have been developed to explain the atmospheric and solar neutrino anomalies. Some models such as sterile neutrinos [60], non-standard interactions [61], Lorentz violation [62], $\nu \rightarrow \bar{\nu}$ transitions [63], have been tested with the MINOS experiment.

\subsection{Outlook}

The data from a variety of solar, atmospheric, accelerator and reactor neutrino experiments provides compelling evidence of neutrino flavor transformation in propagation. This phenomenon is successfully interpreted by the neutrino oscillation model, which indicates that neutrinos have mass. This 
breakthrough is beyond the description of the Standard Model of particle physics. For decades, the experimental data revealed a number of important properties of neutrinos. However we are still far from understanding the nature of this elusive particle which is a crucial issue at the frontier of particle physics, astrophysics and cosmology. In the next few decades, experimental neutrino programs will continue to search for neutrino properties intensively. The precision measurement of these, like the $\mathrm{CP}$ phase and the mass hierarchy, will reveal the mysteries of neutrinos and will be a breakthrough in understanding its nature. The status of experimental neutrino physics and future prospects in this field will be presented in Chapter 2 . 


\section{Chapter 2}

\section{The status of experimental neutrino oscillations}

This chapter describes the experimental programs in neutrino physics. This includes our understanding of oscillation parameters, absolute mass scale

and a brief description of neutrino velocity measurements. The forthcoming results and future prospects are also discussed.

\subsection{Natural neutrino source-based programs}

\subsubsection{Cosmology and supernova neutrinos}

It is widely believed that very soon after the Big Bang, all particles were in thermal equilibrium. Neutrinos were created and annihilated in pairs from their interactions with electrons and positrons by:

$$
e^{+}+e^{-} \Leftrightarrow \nu+\bar{\nu}
$$

When the Universe expanded, the temperature dropped, making it difficult for neutrinos to create electrons since they did not have enough energy. Eventually, once the mean time for the inverse interaction $\left(\nu+\bar{\nu} \rightarrow e^{+}+e^{-}\right)$ became longer than the age of the universe, neutrinos effectively decoupled from the other particles, and traveled as a free stream. This stream is called 
the relic neutrino background, which is similar to the cosmic microwave background (CMB). The existence of relic neutrinos, if discovered, would be the most compelling evidence for the standard Big Bang model. By counting the "effective" relativistic degree of freedom of photons and fermions involved in neutrino interactions, a relationship exists between neutrino and photon temperatures [26] as follows:

$$
T_{\nu}=\left(\frac{4}{11}\right)^{1 / 3} T_{\gamma} \approx\left(\frac{4}{11}\right)^{1 / 3} 2.725 \approx 1.95 K
$$

Because of low kinetic energy $\left(E_{\text {kin }}=3 T \approx 5 \times 10^{-4} \mathrm{eV}\right)$, the detection of relic neutrinos is far beyond the capability of the present-day generation of neutrino detectors.

Another important source of cosmological neutrinos is from supernova explosions. In 1987, eleven neutrino events from the supernova SN 1987a were observed by the Kamiokande-II [64] experiment and eight by the IrvineMichigan-Brookhaven (IMB) experiment [65]. These types of neutrinos are crucial to understanding the core-collapse mechanism of star explosion. However, the data recorded from SN 1987a was insufficient to reveal much. The Super-Kamiokande detector, which is 25 times the size of Kamiokande-II, was primarily built to observe a large number of neutrino events when a new supernova explosion occurs.

\subsubsection{Solar neutrinos}

Solar neutrinos are produced by nuclear fusion reactions in the core of the Sun. The energy spectra of solar neutrinos are well calculated by the 
Standard Solar Model (SSM) [66]. The vast majority of solar neutrinos come from the proton-proton reactions, which are comprised of the following five nuclear reactions:

\begin{tabular}{lll}
\hline \hline reactions & $\nu_{e}$ type & $\nu_{e}$ energy \\
\hline & & \\
$p+p \rightarrow d+e^{+}+\nu_{e}$ & $p p$ & $<0.42 \mathrm{MeV}$ \\
$p+e+p \rightarrow d+\nu_{e}$ & $p e p$ & $1.44 \mathrm{MeV}$ \\
$e+{ }^{7} B e \rightarrow{ }^{7} L i+\nu_{e}$ & ${ }^{7} B e$ & $0.85 \mathrm{MeV}(90 \%), 0.38 \mathrm{MeV}(10 \%)$ \\
${ }^{8} B \rightarrow{ }^{8} B e^{*}+e^{+}+\nu_{e}$ & ${ }^{8} B$ & $<15 \mathrm{MeV}$ \\
${ }^{3} \mathrm{He}+p \rightarrow{ }^{4} \mathrm{He}+e^{+}+\nu_{e}$ & $h e p$ & $<18.8 \mathrm{MeV}$ \\
\hline \hline
\end{tabular}

Table 2.1: Five nuclear reactions of the proton-proton chain produce solar neutrinos in the core of the Sun.

Nuclear fusion reactions in the core of the Sun can be effectively expressed as [67]:

$$
4 p \rightarrow{ }^{4} \mathrm{He}+2 e^{+}+2 \nu_{e}+26.731 \mathrm{MeV} .
$$

Although the Sun creates an enormous flux of $4.0 \times 10^{10} \nu_{e} / \mathrm{cm}^{2} / \mathrm{sec}$, $90 \%$ the flux on the surface of the Earth is coming from pp-type neutrinos (see reactions), which are very low energy $(<0.42 \mathrm{MeV})$. The solar neutrinos, which have been used in studying neutrino oscillations, normally are ${ }^{7} \mathrm{Be}-$, ${ }^{8} B-$, and $p e p$-type neutrinos (see reactions). A number of experiments with different techniques have been constructed to study solar neutrinos. A Chlorine apparatus, which takes the advantage of radio-chemical reaction:

$$
\nu_{e}+{ }^{37} \mathrm{Cl} \rightarrow e^{-}+{ }^{37} \mathrm{Ar} \quad\left(E_{\text {threshold }}=0.814 \mathrm{MeV}\right),
$$


was used with the Homestake experiment. Both the SAGE and GALLEX experiments utilize the Gallium radio-chemical reaction:

$$
\nu_{e}+{ }^{71} \mathrm{Ga} \rightarrow e^{-}+{ }^{71} \mathrm{Ge} \quad\left(E_{\mathrm{threshold}}=0.233 \mathrm{MeV}\right)
$$

Another technique, Cherenkov radiation in water, was widely conducted in the Kamiokande, Super-K, SNO, IceCube and ICARUS ${ }^{1}$ experiments [15, 35, 39, 68, 69]. Other experiments such as Borexino ${ }^{2}$ and SNO+ (SNO's successor) $[70,71]$ make use of a high-degree purity liquid scintillator as the target material. The observation of a solar neutrino deficit from these experiments is discussed in Section 1.4.1 and marked a crucial milestone in neutrino physics. The solar neutrino measurements have determined precisely the $\nu_{e}$ survival probability, which is characterized by the solar mixing angle $\theta_{12}$ and the solar mass-squared splitting $\Delta m_{12}^{2}$. These measurements have also provided a crucial test of the Sun's inner core structure models. In the future, measurements with solar neutrinos are expected to explore the matter effect, search non-standard interactions and measure the $\nu_{e}$ survival probability more precisely $[72]$.

\subsubsection{Atmospheric neutrinos}

The Earth is constantly bombarded by cosmic ray particles from space. Cosmic rays are composed of $90 \%$ of hydrogen nuclei, $9 \%$ of helium nuclei $(\alpha$ particles) and $1 \%$ of heavy nuclei with energy up to and more than $1 \mathrm{TeV}$. The

\footnotetext{
${ }^{1}$ Imaging Cosmic And Rare Underground Signals

${ }^{2}$ Boron solar neutrino experiment
} 
protons and heavier nuclei interact with the Earth's atmosphere and produce showers of pions, which subsequently decay to muons and muon neutrinos. If all the muons decayed in to the muon neutrinos and electron neutrinos before reaching the ground, it would be expected that there are two muon neutrinos for each electron neutrino observed:

$$
\frac{\nu_{\mu}+\bar{\nu}_{\mu}}{\nu_{e}+\bar{\nu}_{e}}=2 .
$$

With approximate 15\% uncertainty in the absolute neutrino flux, this ratio is estimated with a precision up to $1 \%$ in the $E_{\nu}<10 \mathrm{GeV}$ region [73].

As discussed in Section 1.4.2, many experiments have made contributions to the measurement of this ratio. The measured ratios are significantly smaller than 2, giving rise to the so-called atmospheric neutrino anomaly. The atmospheric neutrino measurements have provided precisely the $\nu_{\mu}$ survival probability, which is essentially characterized by the atmospheric mixing angle $\theta_{23}$ and atmospheric mass-squared splitting $\left|\Delta m_{32}^{2}\right|$. In the coming era of precision measurement of oscillation parameters, atmospheric neutrino experiments are also expected to provide a great deal of sensitivity to the mass hierarchy due to the large Earth matter effects. To explore this sensitivity, a number of proposals have been conceived. The iron calorimeter detector at the India-based Neutrino Observatory (ICAL@INO), will have great capacity for separating $\nu_{\mu}$ and $\bar{\nu}_{\mu}$ on event-by-event basis. The Hyper-Kamiokande experiment, proposed as a megaton water Cherenkov detector and the multiplemegaton ice Precision IceCube Next Generation Upgrade (PINGU) detector 
will be able to observe large statistics of muon neutrinos and electron neutrinos, which provide an exceptional opportunity to resolve the neutrino mass hierarchy [74].

\section{$2.2 \quad$ Man-made neutrino source-based programs}

When compared to natural source-based programs, the man-made neutrino source-based programs show a number of advantages. They include the capability of controlling the neutrino flavors, limiting background, and substantially reducing systematic uncertainties. In this section, reactor and atmospheric neutrino-based programs are presented and followed up with a discussion of future proposals.

\subsubsection{Reactor neutrinos}

Fission reactors generate energy by breaking heavy nuclei (usually U235) into smaller fragments, which, in turn, decay via a cascade of beta decays into stable nuclei. On average, each fission yields about $200 \mathrm{MeV}$ of energy and 6 electron antineutrinos, $\bar{\nu}_{e}$. The neutrino energies from reactors range from 1 to $10 \mathrm{MeV}$. The favorable feature of the nuclear reactor is a very pure $\bar{\nu}_{e}$ flux, which is known to be within $1 \%$ of absolute uncertainty. Moreover, since the event rate scales with the reactor power, it is simple to predict the number of produced $\bar{\nu}_{e}$. However, their energy spectra, which are more sensitive to a few physical phenomena, are difficult to measure. Another shortcoming of reactor neutrino-based programs is that the flux decreases in proportion to 
$1 / r^{2}$ distance from the reactor. This limits the baseline length for the reactor detector.

The study of oscillation physics with reactor neutrinos mainly focuses on the $\bar{\nu}_{e}$ disappearance in the short baseline. In a vacuum, the full expression of the $\bar{\nu}_{e}$ survival probability is as follows:

$$
\begin{aligned}
P_{\bar{\nu}_{e} \rightarrow \bar{\nu}_{e}}(L / E)= & -\cos ^{4} \theta_{13} \sin ^{2} 2 \theta_{12} \sin ^{2} \frac{\Delta m_{21}^{2} L}{4 E} \\
& -\cos ^{2} \theta_{12} \sin ^{2} 2 \theta_{13} \sin ^{2} \frac{\Delta m_{31}^{2} L}{4 E} \\
& -\sin ^{2} \theta_{12} \sin ^{2} 2 \theta_{13} \sin ^{2} \frac{\Delta m_{32}^{2} L}{4 E} .
\end{aligned}
$$

The measurements from Daya Bay, Double Chooz and other reactor experiments $[17,47]$ provide a precise constraint on the mixing angle $\theta_{13}$. The discovery of a non-zero $\theta_{13}$ [17] in 2012, which marked a crucial milestone in neutrino physics, opens the opportunity to measure the $\mathrm{CP}$ violation in the lepton sector and the mass hierarchy of neutrinos.

In the future, while reactor neutrino-based experiments continue to improve sensitivity to determine the mixing angle $\theta_{13}$, a study of mass hierarchy in reactor neutrinos will be considered by looking for interference between the two oscillation frequencies driven by $\Delta m_{31}^{2}$ and $\Delta m_{32}^{2}$, as illustrated in Figure 2.1. 


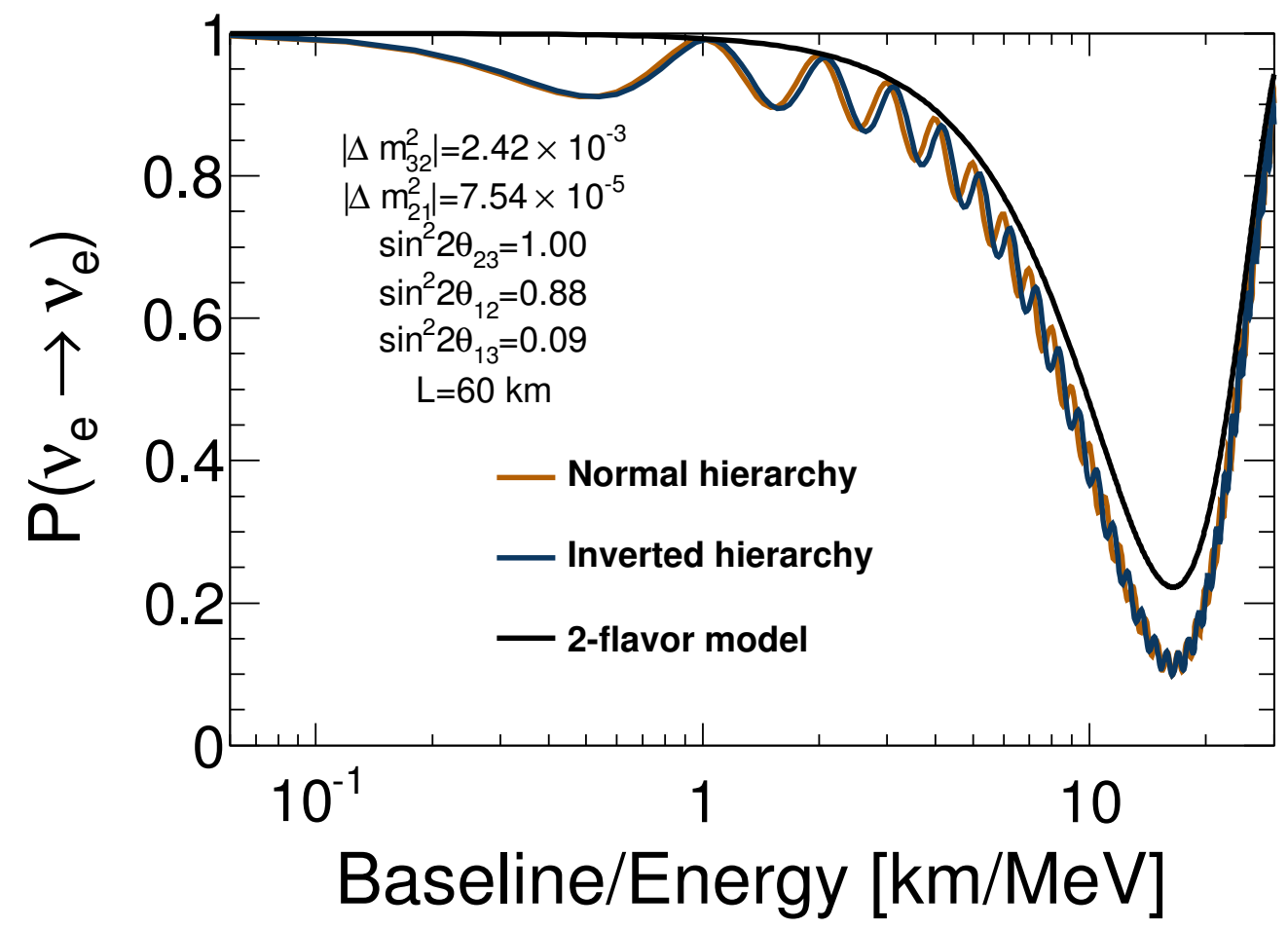

Figure 2.1: The $\nu_{e}$ survival probability shows two oscillation frequencies driven by $\Delta m_{31}^{2}$ and $\Delta m_{32}^{2}$. The normal hierarchy and inverted hierarchy cases are shown respectively in orange and blue lines. The approximate two-flavor probability, a limit $\frac{\Delta m_{31}^{2} L}{E} \simeq 0$ and $\Delta m_{31}^{2} \simeq \Delta m_{32}^{2}$ of Eq. (2.7), is presented by black line.

\subsubsection{Accelerator neutrinos}

The usual muon neutrino beam from a proton accelerator is produced as follows: The proton beam is accelerated and aimed at a target, producing a large number of secondary pions $\pi^{ \pm}$, kaons $K^{ \pm}$and other particles. A number of focusing horns with shaped magnetic fields allow one to select momentumdetermined $\pi^{ \pm} / K^{ \pm}$, which decay into muon neutrinos or muon antineutrinos, 
$\nu_{\mu}\left(\bar{\nu}_{\mu}\right)$, when traveling through a decay pipe. Hadron absorbers are inserted at the end of the decay pipe to absorb all hadrons, resulting in a nearly pure beam of $\nu_{\mu}\left(\bar{\nu}_{\mu}\right)$.

The absolute accelerator neutrino flux is determined with a large degree of uncertainty, up to $20 \%$, which is mainly inherited from the not well-

understood $\pi^{ \pm} / K^{ \pm}$production in proton-target interactions [75]. To mitigate this uncertainty, the accelerator neutrino-based experiments are usually constructed with two detectors. The Near Detector, located near to the neutrino sources, is used to measure the neutrino flux before oscillation and the Far Detector, downstream from the neutrino source, collects the data after neutrino flavor transformation has occurred. The accelerator beams are widely divided into two types:

\section{Wide-band neutrino beam}

The broad energy spectrum of this beam type allows one to observe simultaneously the first and second maximum oscillation nodes in the $\nu_{\mu}\left(\bar{\nu}_{\mu}\right)$ disappearance channel. This unique aspect offers a promising potential for solving the degeneracy of neutrino parameters [76]. This normally requires a very powerful beam along with a very long baseline. However, this baseline reduces the sensitivity to measure the mixing angle $\theta_{13}$ and the $\mathrm{CP}$ phase because of low event rate observed at the Far Detector. 


\section{Off-axis neutrino beam}

This beam type has lower intensity, approximately 100 times lower in comparison to the intensity of the wide-band beam. The favorable feature of this beam type is the precise prediction of neutrino spectrum, which is crucial for a more accurate measurement of oscillation parameters. Moreover, studying the kinematics of the neutrino beam [77] shows that the off-axis component of a neutrino beam has a sharper peak of energy distribution than the on-axis component, as illustrated in Figure 2.2. This critical asset is utilized in the world's first two off-axis neutrino experiments, the T2K and NOvA experiments. The neutrino physics with the narrow-band beam comes from four interested channels coupled into two pairs: (i) $\nu_{\mu} / \bar{\nu}_{\mu}$ disappearances and (ii) $\nu_{e} / \bar{\nu}_{e}$ appearances. While the former produces the most stringent constraint of the atmospheric mass-squared splitting and examines the maximality of the mixing angle $\theta_{23}$, the latter is used to measure the mixing angle $\theta_{13}$, mass hierarchy and sheds some light on the CP phase, briefly discussed in Appendix D. The T2K experiment has recently reported evidence of a non-zero mixing angle $\theta_{13}$ with $7.3 \sigma$ significance [18]; the NOvA experiment, known as the world's longest-distance neutrino experiment, is on the way to complete installation of the Far Detector. In the next few years, results from these two experiments will probably reach a new realm in neutrino physics. 

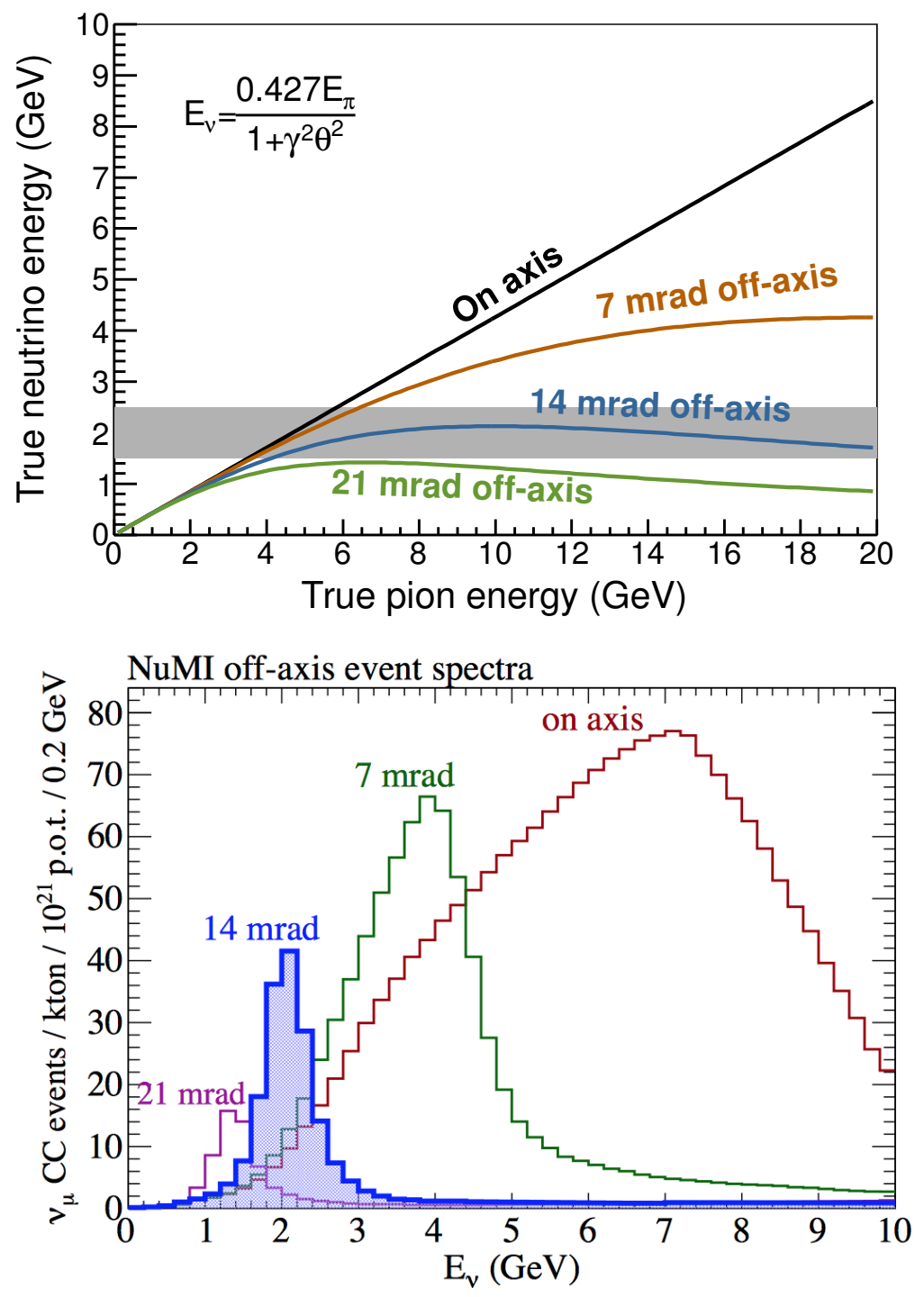

Figure 2.2: The top plot shows the relationship between the pion energy and the energy of induced neutrinos with different angles between them. The simulated energy spectra of $\nu_{\mu}$ charged-current events in the detector with different angles (0, $7 \mathrm{mrad}, 14 \mathrm{mrad}$, and $21 \mathrm{mrad}$ ) off from the center of NuMI beam (introduced in Section 3.3) are shown at the bottom. The NO $\nu \mathrm{A}$ detector lies at $14 \mathrm{mrad}$ for optimizing sensitivity to the $\nu_{e}$ appearance. The bottom plot is taken from [50]. 


\subsubsection{Future of neutrino beams}

The new generation off-axis experiments like T2K and NOvA would

provide highly precise measurements of mixing angle $\theta_{23}, \Delta m_{23}^{2}$ and even $\theta_{13}$, but they barely solve definitely the mass hierarchy and the CP-violating phase, because they are buried in the degeneracy and correlation among oscillation parameters. The matter effect is found to be the most powerful tool for solving this question. However, in order to enhance the matter effect, a necessary but crucial feature is a very long baseline, which can dramatically reduce the event rate. To solve this drawback, updating beam intensity of our current neutrino beam to a so-called super-beam is considered. Compared to the current $700 \mathrm{~kW}$-powered operation of the NuMI beam (see Section 3.3), the super-beam power is expected to be in the range of 2-5 MW. In addition to the super-beam, a proposal for a neutrino factory is also envisaged. This is the ultimate accelerator-driven neutrino beam, which generates neutrinos by the decay of muons stored in a particle accelerator $\mu^{ \pm} \rightarrow e^{ \pm}+\nu_{e}\left(\bar{\nu}_{e}\right)+\nu_{\mu}\left(\bar{\nu}_{\mu}\right)$. This decay is extremely simple and well-understood. Additionally, the beam from the neutrino factory consists of only muon-type and electron-type neutrinos, which can be distinguished in a straightforward manner. The most challenging part of constructing a neutrino factory is the $2.2 \mu \mathrm{s}$ life-time of muons and a sophisticated approach for forming a well-collimated beam from random muon motions. Many interesting neutrino physics with a super-beam and neutrino factory are discussed in [76]. 


\subsection{Experimental status of neutrino mixing}

\subsubsection{Solar sector}

The neutrino oscillation measurements involving the smaller of the two mass-squared splittings, $\Delta m_{21}^{2}$, is often referred to as the solar sector measurement. This sector dominates neutrino oscillations at the order of $10^{5} \mathrm{~km} / \mathrm{GeV}$ for the ratio of distance propagated to neutrino energy. The measurements in this sector come from the solar neutrino source-based experiment, as discussed in Section 2.1.

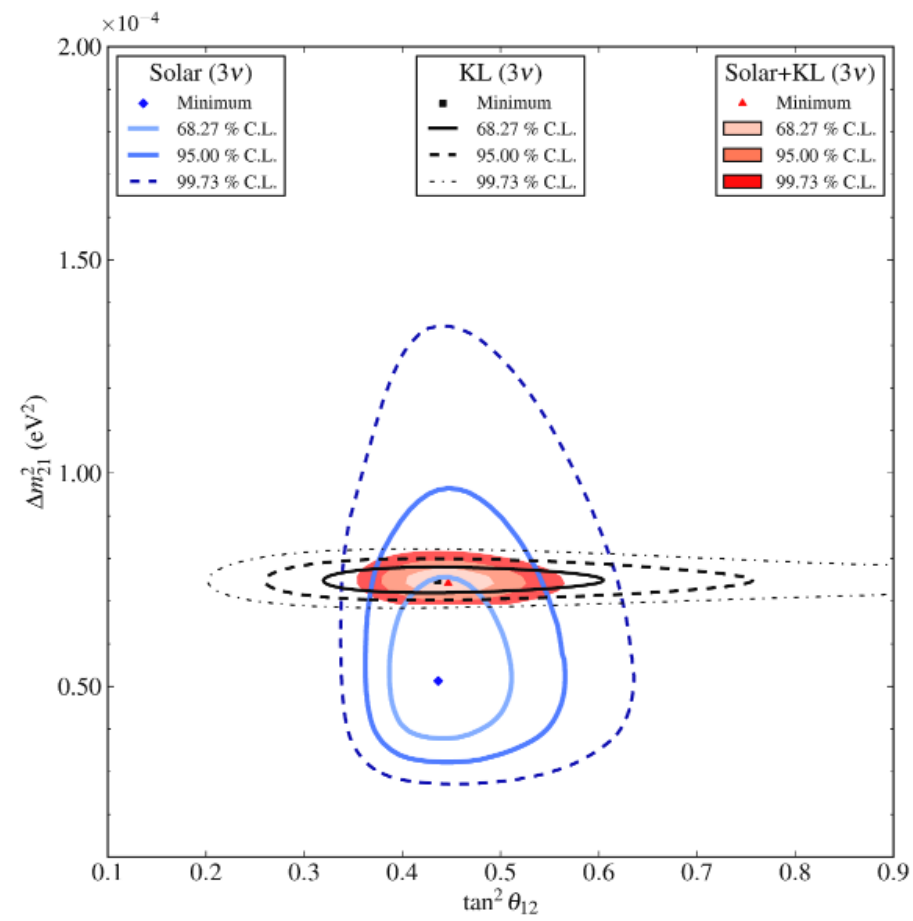

Figure 2.3: Best limits of oscillation parameters in the solar neutrino sector come from the global fitting with combined solar neutrino and KamLAND data set. Figure taken from [78]. 
The short baseline-based experiments, like KamLAND, also have made a substantial contribution to this sector since the atmospheric term would be averaged out due to reconstructed energy resolution, see Eq. (1.25). The global fitting [78] of the combination of the solar neutrino and KamLAND data set yields the most stringent constraint in the solar sector as follows:

$$
\Delta m_{21}^{2}=7.59_{-0.21}^{+0.20} \times 10^{-5} \mathrm{eV}^{2} \text { and } \theta_{12}=\left(34.06_{-0.84}^{+1.16}\right)^{o} .
$$

Figure 2.3 shows the confidence level (C.L.) surfaces of oscillation parameters with separated solar neutrino and KamLAND data sets along with a combined set in the solar sector.

\subsubsection{Atmospheric sector}

The neutrino oscillation measurements involving the larger of the two mass-squared splittings, $\Delta m_{32}^{2}$, is often known as the atmospheric sector measurement. This sector governs the neutrino oscillation at the order of $10^{3} \mathrm{~km} / \mathrm{GeV}$ for the ratio of distance propagated to neutrino energy. The best constraints come from measuring the $\nu_{\mu} \rightarrow \nu_{\mu}$ channel, see Eq. (1.23). The MINOS experiment [79] gives the best measurement of this mass splitting, while the best measurement of the mixing angle belongs to the Super-K experiment [80]. Figure 2.4 shows the $90 \%$ C.L. constraints on the atmospheric oscillation parameters from the MINOS, Super-K and T2K experiments.

$$
\begin{aligned}
& \text { MINOS: }\left|\Delta m_{32}^{2}\right|=2.41_{-0.10}^{+0.09} \times 10^{-3} e V^{2}, \quad \sin ^{2}\left(2 \theta_{23}\right)>0.89(90 \% \text { C.L }) \\
& \text { Super-K: }\left|\Delta m_{32}^{2}\right|=2.11_{-0.19}^{+0.11} \times 10^{-3} e V^{2}, \quad \sin ^{2}\left(2 \theta_{23}\right)>0.96(90 \% \text { C.L })
\end{aligned}
$$




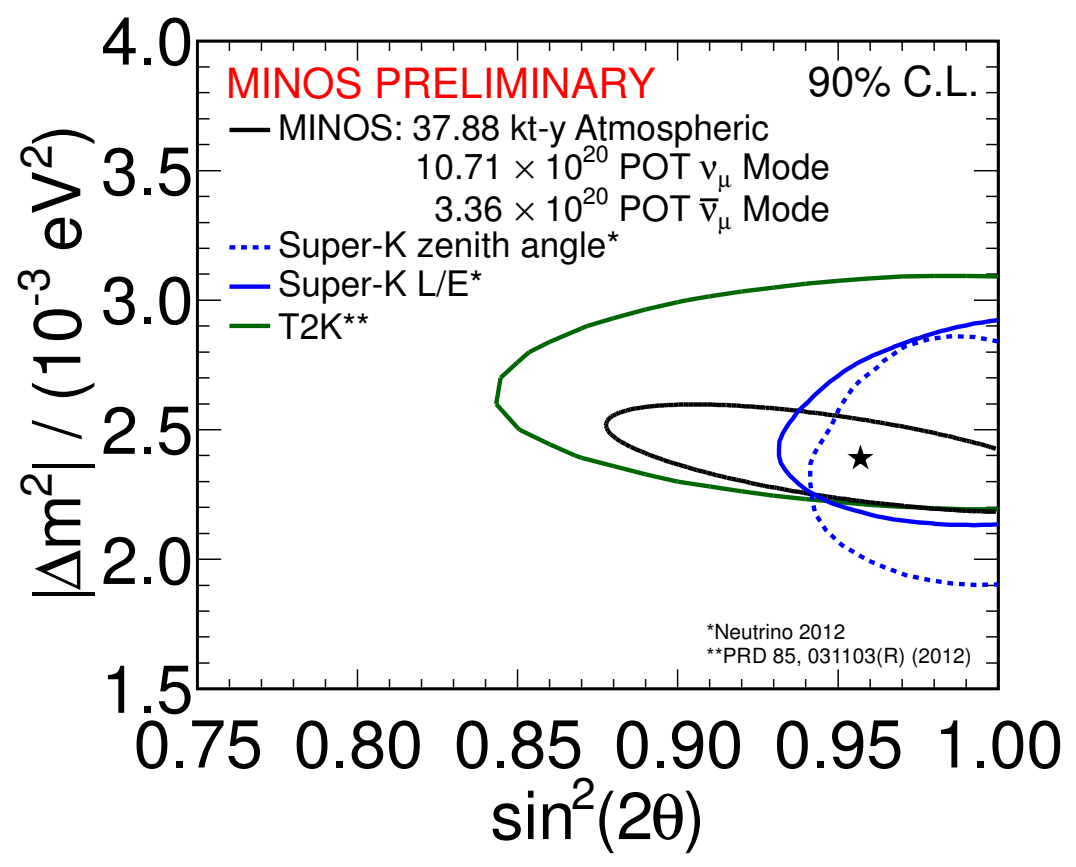

Figure 2.4: Best limits of oscillation parameters in the atmospheric neutrino sector. Figure taken from [79].

\subsubsection{Tau neutrino appearance from muon neutrino beam}

The $\nu_{\mu}$ disappearance from the $\nu_{\mu}$ beam is predominantly due to transisistions into $\nu_{\tau}$. However the $\nu_{\tau}$ appearance is barely observed due to the massiveness and short life-time of tau particles. The Oscillation Project with Emulsion-tRacking Apparatus (OPERA) experiment, which uses a $\nu_{\mu}$ beam produced at $\mathrm{CERN}^{3}$ and observes neutrino interaction in a detector $730 \mathrm{~km}$ way from target, has been built to facilitate this measurement. Until 2013, the OPERA experiment observed three $\nu_{\tau}$ candidate events [81].

\footnotetext{
${ }^{3}$ The European Organization for Nuclear Research.
} 


\subsubsection{Mixing angle $\theta_{13}$}

Although most $\nu_{\mu}\left(\bar{\nu}_{\mu}\right)$ are oscillated to $\nu_{\tau}\left(\bar{\nu}_{\tau}\right)$, a small portion of $\nu_{\mu}\left(\bar{\nu}_{\mu}\right)$ are believed to be converted into $\nu_{e}\left(\bar{\nu}_{e}\right)$. The magnitude of this portion is governed by the mixing angle $\theta_{13}$. The importance of measuring this mixing angle lies in the fact that $\mathrm{CP}$ violation in the lepton sector can only be observed if $\theta_{13}$ is non-zero. In 2012, the Daya Bay experiment [17], a reactor neutrinobased program discussed in Section 2.2, announced a $7.7 \sigma$ significant discovery for a non-zero $\theta_{13}$ :

$$
\sin ^{2} 2 \theta_{13}=0.089 \pm 0.010 \text { (stat.) } \pm 0.005 \text { (syst.). }
$$
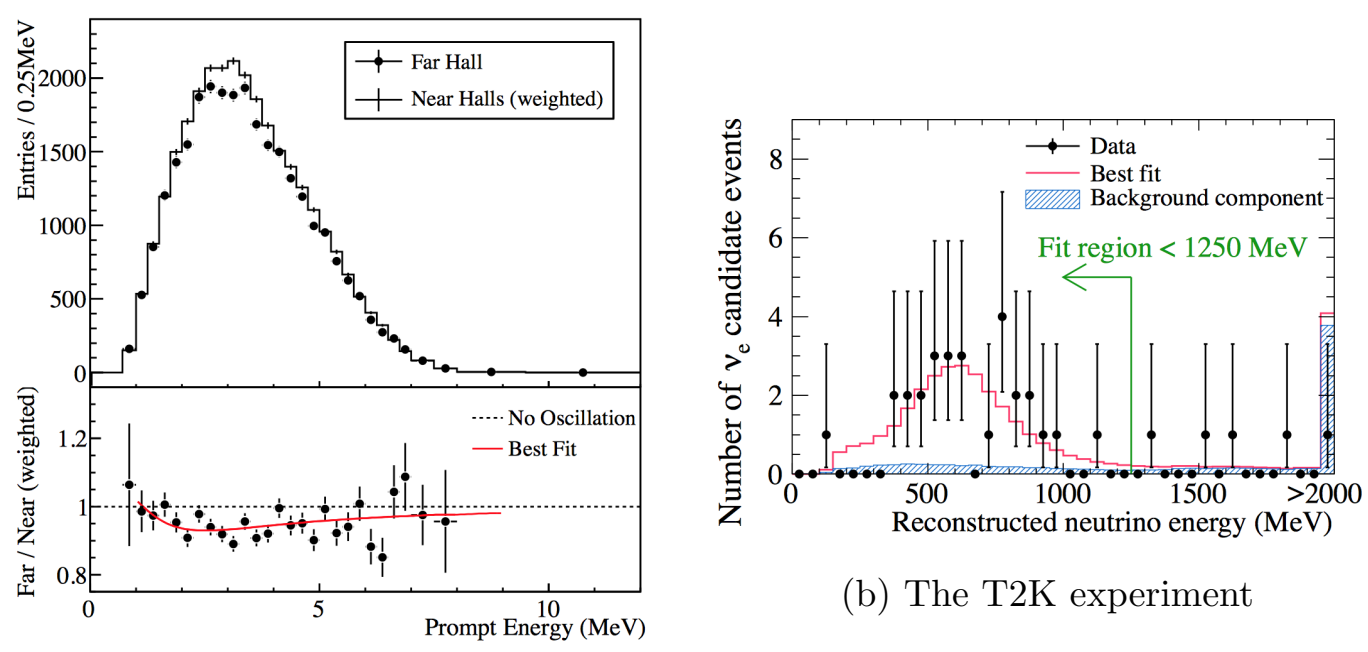

(a) The Daya Bay experiment

Figure 2.5: The substantial signals of $\bar{\nu}_{e}$ appearance in the Daya Bay experiment (left) and $\nu_{e}$ appearance in the T2K experiment (right). Figure taken from [17] and [18]. 
In 2013, the T2K experiment [18] confirmed the relatively large value of mixing angle $\theta_{13}$ with a significance of $7.3 \sigma$. Figure 2.5 shows the substantial signal of $\bar{\nu}_{e}$ appearance in the Daya Bay and $\nu_{e}$ appearance in the T2K experiments.

\subsubsection{Sterile neutrinos}

In the 1990s, the Liquid Scintillator Neutrino Detector (LSND) experiment reported a neutrino transition driven by a mass-squared splitting of the order of $1 \mathrm{eV}^{2}$ [82]. This claim implied the existence of a fourth type of

neutrinos since it differed from atmospheric sector scale of $10^{-3} \mathrm{eV}$ and solar sector scale of $10^{-5} \mathrm{eV}$. However, measurement of the decay width of $\mathrm{Z}$ boson shows good agreement with the widely-known model of only three light active neutrino flavors [10]:

$$
N_{\nu}^{\text {active }}=2.9840 \pm 0.0082
$$

Also the constraints from cosmology [11] for the number of neutrino species yield, favors three generations:

$$
N_{\nu}^{\mathrm{eff}}=3.52_{-0.45}^{+0.48} \quad(95 \% \text { C.L.; Planck }+\mathrm{WP}+\text { highL }+ \text { BAO })
$$

Therefore any additional neutrinos are believed to be sterile in a sense that they do not interact through the weak force. 

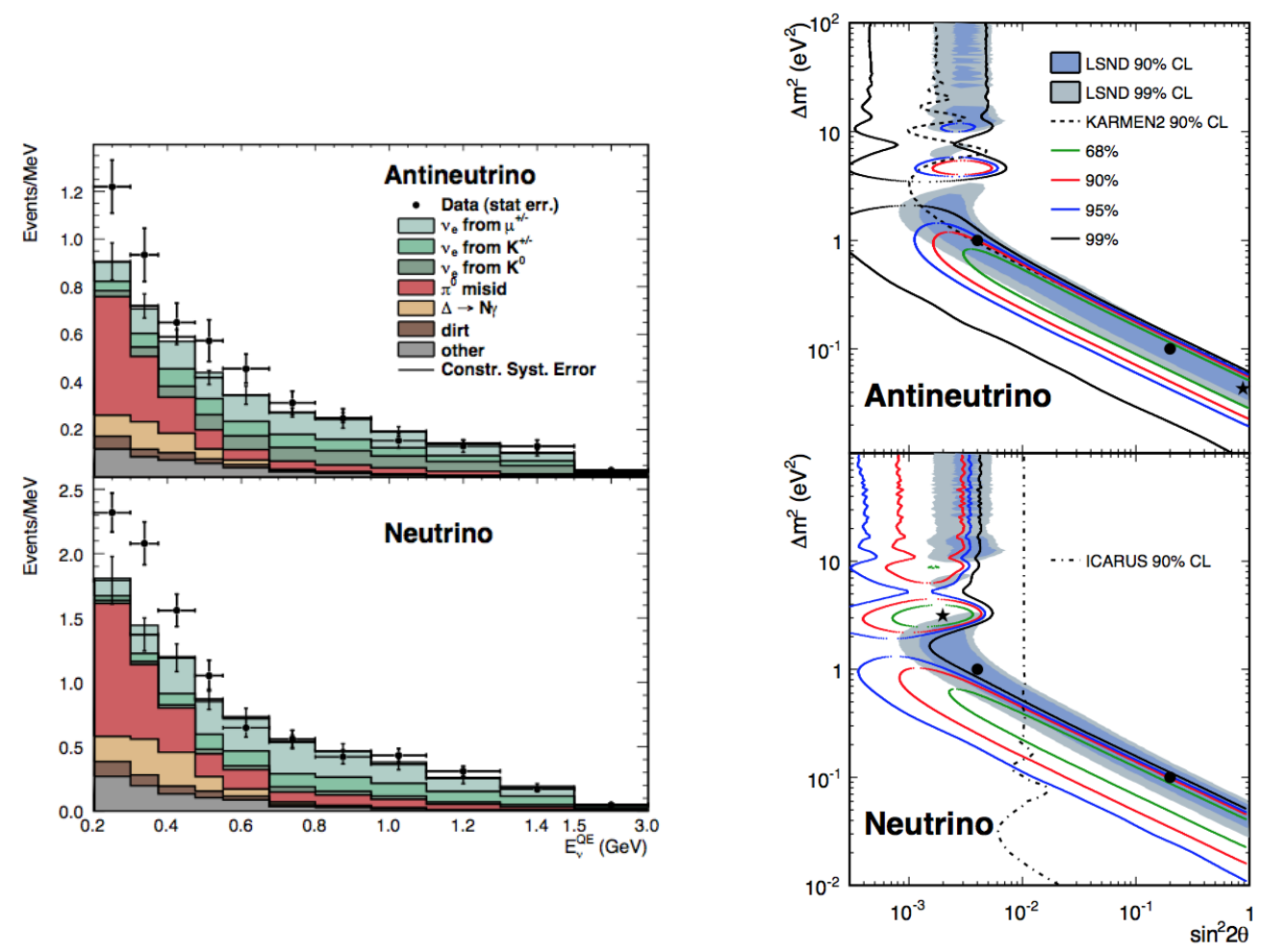

Figure 2.6: The $E_{\nu}^{\mathrm{QE}}$ distributions (left) for antineutrino (top) and neutrino (bottom) and the MiniBooNE allowed regions (right). Figure taken from [83].

The MiniBooNE ${ }^{4}$ experiment was constructed to test the LSND anomaly. Their data were consistent with $\bar{\nu}_{e}$ oscillation in the $0.01<\Delta m^{2}<1.0 \mathrm{eV}^{2}$ range and overlapped partly with the LSND results, as shown in Figure 2.6. Transformations of active neutrinos to sterile neutrinos (a controversial idea) was used as an explanation. Also it is possible that the $\bar{\nu}_{e}$ flux is not wellunderstood and needs to be re-evaluated [84].

\footnotetext{
${ }^{4}$ BooNE is an acronym for the Booster Neutrino Experiment
} 


\subsection{Experimental status of absolute neutrino mass}

Neutrino oscillations tell us nothing about the absolute neutrino mass scale. The limits of the absolute scale of neutrino mass are constrained by beta decay, neutrinoless double beta decay, and cosmology.

\subsubsection{Supernovae and cosmological constraints}

Supernovae are copious sources of neutrinos. By measuring the neutrino speed, in principle, it is possible to constrain neutrino masses down to $m_{\nu_{e}}<$ $12 \mathrm{eV} / \mathrm{c}^{2}$, like SN1987A [85]. Since neutrinos are extremely numerous in the Universe, the cosmological effects of their even-tiny mass can be observed and used to set limits on total neutrino mass. The recent data from Planck [11] yields:

$$
\left.\sum m_{\nu}<0.23 e V \quad \text { (95\% C.L.; Planck }+\mathrm{WP}+\text { highL }+\mathrm{BAO}\right) .
$$

However these constraints are fairly model-dependent and cannot take place of the direct measurements in laboratory experiments.

\section{Beta decay}

The effective mass of $\nu_{e}$ can be measured via the beta-decay by looking at the endpoint of the beta-decay spectrum. The current limit from the MainzTroitsk neutrino mass experiment [86] on the effective $\nu_{e}$ mass is:

$$
m_{\nu_{e}}=\sqrt{\sum_{i=1}^{3}\left|U_{e i}\right|^{2} m_{i}^{2}}<2.3 \mathrm{eV} .
$$




\subsubsection{Neutrinoless double beta decay}

This decay, if observed, provides the exclusive information regrading the question of neutrino mass nature. This decay is also an excellent tool for measuring the absolute scale of neutrino mass. The effective mass of neutrinos measured from this kind of experiments is:

$$
\left\langle m_{\beta \beta}\right\rangle=\left|\sum_{i=1}^{3} U_{e i}^{2} m_{\nu i}\right| .
$$

KamLand-Zen ${ }^{5}$ [87] reports $\left\langle m_{\beta \beta}\right\rangle<(0.12-0.25) e V$ while Enriched Xenon Observatory (EXO-200) [88] sets the upper limit as $\left\langle m_{\beta \beta}\right\rangle<(0.14-0.38) \mathrm{eV}$.

\subsection{Experimental status of neutrino velocity}

The measurement of neutrino velocity helps to test the relativistic energy-momentum dispersion relationship. In September 2011, the OPERA experiment claimed an observation of superluminal neutrinos from the data collected at the Gran Sasso laboratory, $730 \mathrm{~km}$ downstream away from CERN. However, this observation was subsequently found to be wrong and OPERA later reported their corrected result of neutrino velocity, which is consistent with the speed of light. In response to this claim, the MINOS experiment updated their timing system with a number of studies, combined with a statistical increase by a factor of 8.5 in collected neutrino data, in order to revisit the measurement of neutrino velocity with a more accurate result.

\footnotetext{
${ }^{5}$ KamLAND's successor
} 
An Auxiliary Detector (AD) was introduced as a powerful tool to reduce the electronic latencies of the MINOS detectors. The author of this thesis has installed two ADs at the Near and Far sites and performed analysis on the data collected by these detectors. More detailed information about the AD and measurement of neutrino velocity in MINOS is included in Appendix E.

Table 2.2 shows the most updated results of neutrino propagation time, $t_{\nu}$, deviated from the nominal time-of-flight $\tau, \delta=t_{\nu}-\tau$, from a number of long-baseline neutrino experiments in the world. The neutrino velocities, calculated by applying the conversion $v_{\nu}=L /(\tau+\delta)$ from these measurements, are consistent with the speed of light.

\begin{tabular}{|l|c|}
\hline Experimenst & $\nu$ time of flight $\left(\delta=t_{\nu}-\tau\right)(\mathrm{ns})$ \\
\hline \hline Borexino & $2.7 \pm 1.2$ ( stat.) \pm 3 ( syst.) \\
\hline ICARUS & $0.1 \pm 0.7$ ( stat.) \pm 2.4 ( syst.) \\
\hline LVD & $2.9 \pm 0.6($ stat. $) \pm 3$ ( syst.) \\
\hline OPERA & $1.6 \pm 1.1$ ( stat. $)_{-3.7}^{+6.1}$ (syst.) \\
\hline MINOS & $-2.4 \pm 0.1$ ( stat.) \pm 2.6 ( syst.) \\
\hline
\end{tabular}

Table 2.2: Measurements of deviation from the nominal time-of-flight from number of long-baseline neutrino experiments in the world. 


\subsection{Future prospects of neutrino oscillation experiments}

Of the seven parameters describing the neutrino oscillations phenomena: three mixing angles, two mass-squared splittings, CP phase and the mass hierarchy, the first five parameters have been fairly well-measured to date. The PMNS matrix, which governs the neutrino mixing as discussed in Section 1.4.3, is not completely filled by our current experiment. The up-to-date knowledge of this matrix is discussed in Appendix F. In the next steps, the neutrino experiments will focus on the determination of CP phase and the resolution of mass hierarchy. In addition, precision measurement of $\theta_{13}$ as well as searching for sterile neutrinos are also considered.

\section{$\theta_{13}$ precision measurement}

After the discovery of non-zero mixing angle $\theta_{13}$ using the Daya Bay reactor detector, the on-going accelerator-driven $\mathrm{T}_{2} \mathrm{~K}^{6}$ and NOvA experiments will measure this mixing angle from studies of $\nu_{\mu}\left(\bar{\nu}_{\mu}\right) \rightarrow \nu_{e}\left(\bar{\nu}_{e}\right)$ transitions. These measurements not only set stringent constraints on $\theta_{13}$ mixing angle but also facilitate investigations regarding the CP phase.

\section{Mass hierarchy resolution}

The two mass-squared splittings from atmospheric sector and solar sector are well-estimated. However, the mass hierarchy of neutrinos is still un-

\footnotetext{
${ }^{6} \mathrm{~T} 2 \mathrm{~K}[18]$ has recently reported evidence of a non-zero mixing angle $\theta_{13}$ at $7.3 \sigma$ significance.
} 
known. The matter effect is the key to resolve this ambiguity. To have significant contribution from the matter effect, this kind of experiment requires a very-long baseline. No current baseline neutrino experiment has had a significant enough contribution of matter effect to definitely measure the sign of mass-squared splitting. The accelerator-driven experiments such as NOvA and the proposed LBNE would have longer baselines and higher intensities in order to gain more sensitivity to the mass hierarchy. Also, the incoming results from huge neutrino telescopes, like Hyper-K and PINGU, with a baseline on the order of the Earth diameter, would be a big step toward resolving the neutrino mass hierarchy.

\section{Measurement of CP phase}

The non-zero $\theta_{13}$ mixing angle is a necessary condition for experimental physicists to test the $\mathrm{CP}$ violation in the lepton sector. The matter effect is the essential for improving sensitivity of the CP-violation search. Since the capability of mass hierarchy resolution also depends on the matter effect, the even longer baseline experiments are required for this search. The T2K (295 $[\mathrm{km}] / 0.6[\mathrm{GeV}]$ of the ratio $\left.L / E_{\nu}\right)$ and NOvA experiments $(810[\mathrm{~km}] / 2 .[\mathrm{GeV}]$ of the ratio $\left.L / E_{\nu}\right)$ might shed some light on the CP phase by comparing the $\nu_{e}$ and $\bar{\nu}_{e}$ appearances from the corresponding $\nu_{\mu}$ and $\bar{\nu}_{\mu}$ beam. However, in order to have a definite answer for CP phase, very long baseline accelerator-driven neutrino experiments along with a very intensive neutrino beam are needed. 


\section{Search for sterile neutrinos}

The observation of $\nu_{e}\left(\bar{\nu}_{e}\right)$ excesses in short-baseline experiments, reported by the LSND and MiniBooNE experiments, could be debated and explained as the transition from active neutrinos to sterile neutrinos. The MicroBooNE experiment is being built to conclusively investigate these controversial results. The sterile neutrinos can be also researched for in long-baseline experiments, like MINOS [60], via measuring the deficit of energy-independent neutral-current event rates or charged-current event rates at energy regions which are not sensitive to the standard oscillation. 


\section{Chapter 3}

\section{The MINOS experiment}

The MINOS experiment is a two-detector long baseline acceleratorbased experiment that uses the world's most intense neutrino beam (the NuMI beam) to study neutrino oscillations. This chapter opens by discussing the physics of accelerator neutrino experiments as well as the physics goals of the MINOS experiment. Then, the NuMI beam and detector technologies are described in detail. After that, the calibration and Monte Carlo simulation procedures in MINOS are presented. Although the MINOS experiment finished in April 2012, these two detectors are used in a new beam configuration in an experiment called MINOS+. The discussion of the neutrino physics with MINOS+ is placed towards the end of this chapter.

\subsection{Accelerator-based neutrino experiments}

The idea of using proton accelerators for studying neutrinos was proposed independently by Pontecorvo [45] and Schwartz [89] in the 1960s. The protons, accelerated to nearly the speed of light, smash into a target and

produce a number of short-lived mesons $\left(\pi^{ \pm} / K^{ \pm}\right)$which decay into muon 
neutrinos or muon antineutrinos as follows:

$$
\begin{aligned}
\pi^{ \pm} \rightarrow \mu^{ \pm}+\nu_{\mu}\left(\bar{\nu}_{\mu}\right) & \mathrm{BR} \approx 100 \% \\
K^{ \pm} \rightarrow \mu^{ \pm}+\nu_{\mu}\left(\bar{\nu}_{\mu}\right) & \mathrm{BR} \approx 63.4 \%
\end{aligned}
$$

The high energy of the mesons guarantees that the produced neutrinos are in a direction close to those of their parent mesons, yielding highly focused neutrino beams.

One of the world's premiere particle accelerators built using this idea was the Alternating Gradient Synchrotron (AGS), which received $200 \mathrm{MeV}$ protons from Brookhaven's linear accelerator (LINAC). Furthermore, one of the most important milestone of the AGS was the discovery of the muon neutrinos in 1962 [6].

Continuing from the success of the AGS, many accelerator-driven neutrino beam facilities have been built at different locations around the world, including Brookhaven, CERN, Fermilab, KEK, Los Alamos, Serpukhov and J-PARC. Facilitated by a number of technological improvements, the power of the neutrino beams has increased dramatically. The figures of merit from various accelerator-based neutrino beams, measured in units of protons-ontarget $(\mathrm{POT})$ multiplied by average neutrino energy $\left(\mathrm{POT} \times\left\langle E_{\nu}\right\rangle\right)$ delivered are showed in Figure 3.1. 


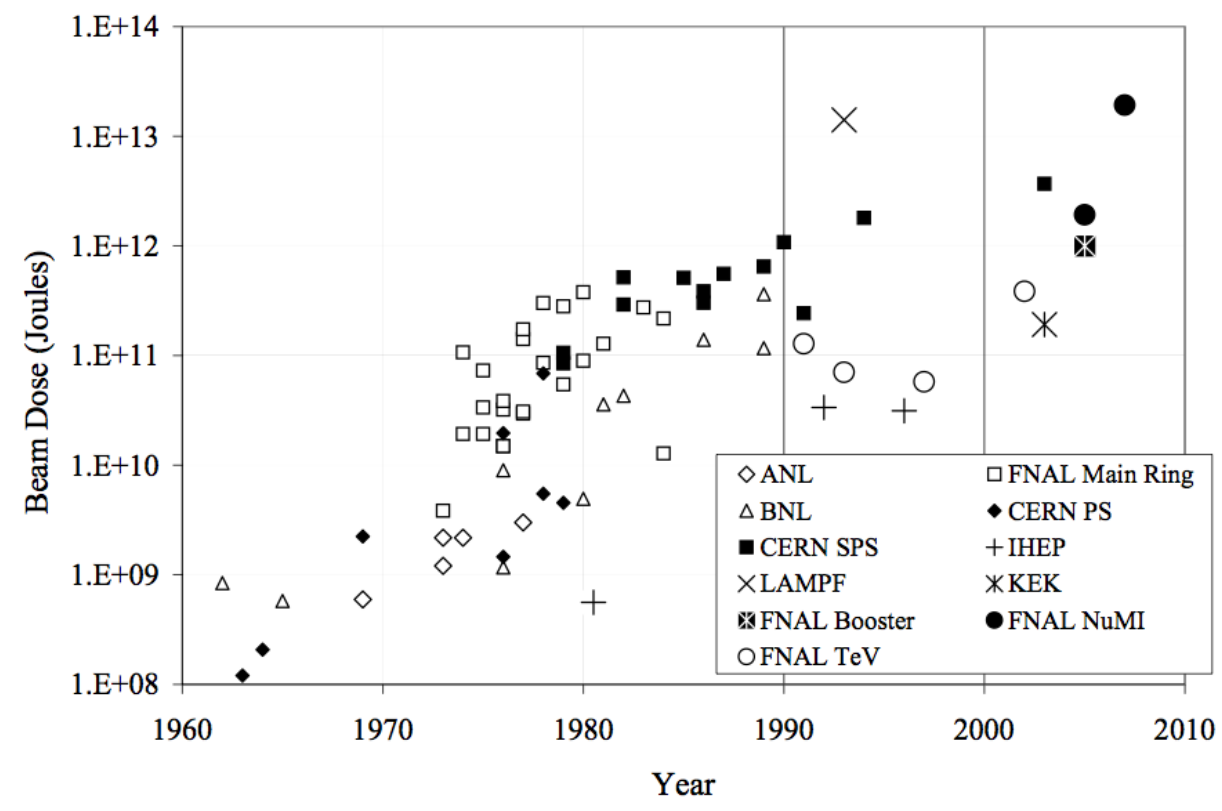

Figure 3.1: The figures of merit $\left(\mathrm{POT} \times\left\langle E_{\nu}\right\rangle\right)$ of the accelerator-based neutrino beams from various laboratories. Figure taken from [75].

The highly pure $\nu_{\mu}$ beams with a flexible energy range of options from the accelerator-based neutrino experiments facilitate the exploration of many crucial areas of neutrino physics. A number of physics goals can be achieved with experiments looking at the $\nu_{\mu}$ disappearance or the $\nu_{e}$ and $\nu_{\tau}$ appearance from $\nu_{\mu}$ transitions. The $\nu_{\mu}$ disappearance is used for precision measurement of atmospheric neutrino oscillation parameters: $\left|\Delta m_{32}^{2}\right|$ and the mixing angle $\theta_{23}$; while the $\nu_{e}$ appearance is sensitive to $\theta_{13}$, mass hierarchy and $\delta_{\mathrm{CP}}$. The appearance of $\nu_{\tau}$ provides a crucial evidence for the oscillation theory, but it is hard to make a precise measurement of this channel due to the limitation of observing $\nu_{\tau}$ events. 


\subsection{Physics goals of MINOS}

The MINOS experiment [90] was designed to make precision measurements of the atmospheric neutrino oscillation parameters, previously measured by the IMB, MACRO ${ }^{1}$, Kamiokande, and Soudan-2 experiments [91-94]. With a large number of innovations for event energy reconstruction and event classification (discussed in Section 3.4), MINOS hoped to confront directly and conclusively the atmospheric anomaly. The following are the highlights of neutrino physics with the MINOS experiment:

\section{Disappearance channel}

This channel is used to measure the atmospheric $\left|\Delta m_{23}^{2}\right|$ and $\sin \theta_{23}$ parameters via the $\nu_{\mu}$ disappearance. In order to make precise measurements, the clean sample of $\nu_{\mu}$ charged-current $(\mathrm{CC})$ events must be selected from the data. The signature of this type of event is a long muon track, with the main background arising from the neutral-current (NC) interactions, which produce a charged pion track in the detectors. The large fiducial volume of the MINOS detectors (0.98 kton of the Near Detector and 5.4 kton of the Far Detector) facilitates this event separation. Also, two sampling steel-scintillator tracking calorimeters allow one to reconstruct the total energy for each event. This is crucial for observing energy-dependent $\nu_{\mu}$ survival probability.

Furthermore, the two-detector setup in MINOS gives a powerful tool to reduce a number of systematic uncertainties in the neutrino flux and the

\footnotetext{
${ }^{1}$ Monopole, Astrophysics and Cosmic Ray Observatory.
} 
detector responses. The Near Detector, $1 \mathrm{~km}$ away from the interaction target, plays a role in the determination of the event spectrum before oscillation. The Far Detector, which observes neutrino events $735 \mathrm{~km}$ away from the neutrino source, expects to see the energy-dependent $\nu_{\mu}$ deficit in comparison to the non-oscillated prediction made by the Near Detector. This deficit is fitted to the $\nu_{\mu}$ survival probability and yields constraints on the atmospheric oscillation parameters.

\section{Appearance channel}

This channel looks for the $\nu_{e}$ appearance due to the subtle $\nu_{\mu} \rightarrow \nu_{e}$ transitions. The pure samples of $\nu_{e}$-CC events are needed for this analysis. The main feature of this type of event is an electromagnetic shower, with the

main background arising from the $\mathrm{NC}$ interactions that produce a number of neutral pion showers in the detectors. Since the MINOS detectors do not have fine grain resolution to easily distinguish these types of events, a sophisticated technique has been developed to select $\nu_{e}$-CC events. The results from this channel help to set a constraint on the mixing angle $\theta_{13}$, study the neutrino mass hierarchy, and shed some light on the CP-violating phase.

\section{Search for sterile neutrino}

This search can be done by comparing the NC interaction rates recorded at the Near Detector and the Far Detector. These rates are not expected to change under the neutrino oscillations in the current widely-accepted three- 
flavor neutrino model. Thus, any difference between the rates of NC events at the two detectors would indicate a physics beyond this model, i.e., the existence of a new neutrino type.

\section{Direct measurement for antineutrino oscillation parameter}

The flexibility of NuMI beam allows a $\bar{\nu}_{\mu}$ beam. The MINOS detectors are magnetized and therefore able to distinguish $\bar{\nu}_{\mu}$ from $\nu_{\mu}$, giving MINOS an unique opportunity to measure the antineutrino oscillation parameters from a $\bar{\nu}_{\mu}$ disappearance in the Far Detector. Consequently, this allows MINOS to directly test if $\nu_{\mu}$ and $\bar{\nu}_{\mu}$ obey the same oscillation model. This turns out to be linked directly with the CPT testing in the lepton sector, which is the main theme presented in this dissertation.

\subsection{The NuMI neutrino beam}

The NuMI Facility Project was proposed to produce an intense neutrino beam to meet the demands of a new generation of experiments for the definitive study of neutrino oscillations. The NuMI beam has run since 2005 with a typical power of $350 \mathrm{~kW}$. The NuMI beam uses $120 \mathrm{GeV}$ protons from the Fermilab Main Injector in $10 \mu s$ pulses to produce on average $3 \times 10^{13}$ protons every $2.2 \mathrm{~s}$. First, these accelerated protons smash upon a graphite target and produce a number of secondary particles, including pions and kaons. Next, these mesons are focused in a forward direction by a system of two magnetic horns en route to a decay pipe of $675 \mathrm{~m}$ in length. In this pipe, pions and 
kaons decay into muon neutrinos ${ }^{2}$ and muons. Finally, the produced beam is purified by stopping the hadrons using an absorber, and the muons using approximately $250 \mathrm{~m}$ rock walls, upstream from the Near Detector hall. Figure 3.2 illustrates the schematic of the NuMI beam line. MINOS mainly collects the data with the neutrino energy of $3 \mathrm{GeV}$, optimizing the search for muon neutrino disappearance.

The flexibility of the magnetic horns in the NuMI design, facilitates neutrino production with two different beam configurations: $\nu_{\mu}$-beam mode and $\bar{\nu}_{\mu}$-beam mode. The energy spectra of the neutrino components in these two beams are shown in Figure 3.3. With the horn configured to focus positive mesons, the $\nu_{\mu}$-beam mode, consists of $91.1 \% \nu_{\mu}, 7.1 \% \bar{\nu}_{\mu}$, and $1.8 \% \nu_{e}$ and $\bar{\nu}_{e}$, as shown in Figure 3.3a. While the $\bar{\nu}_{\mu}$-beam mode, operated with the negative mesons-focused configuration horn, has a mixture of $46.8 \% \bar{\nu}_{\mu}, 51.3 \% \nu_{\mu}$, and $1.9 \% \nu_{e}$ and $\bar{\nu}_{e}$, as shown in Figure 3.3b. The preponderant portion of $\nu_{\mu}$ events in the $\bar{\nu}_{\mu}$-beam mode reflects the fact that the cross-sections of $\bar{\nu}_{\mu}$ interactions are between two and three times lower than those of $\nu_{\mu}$ interactions.

\footnotetext{
${ }^{2} \mathrm{~A}$ very small fraction is electron neutrinos.
} 


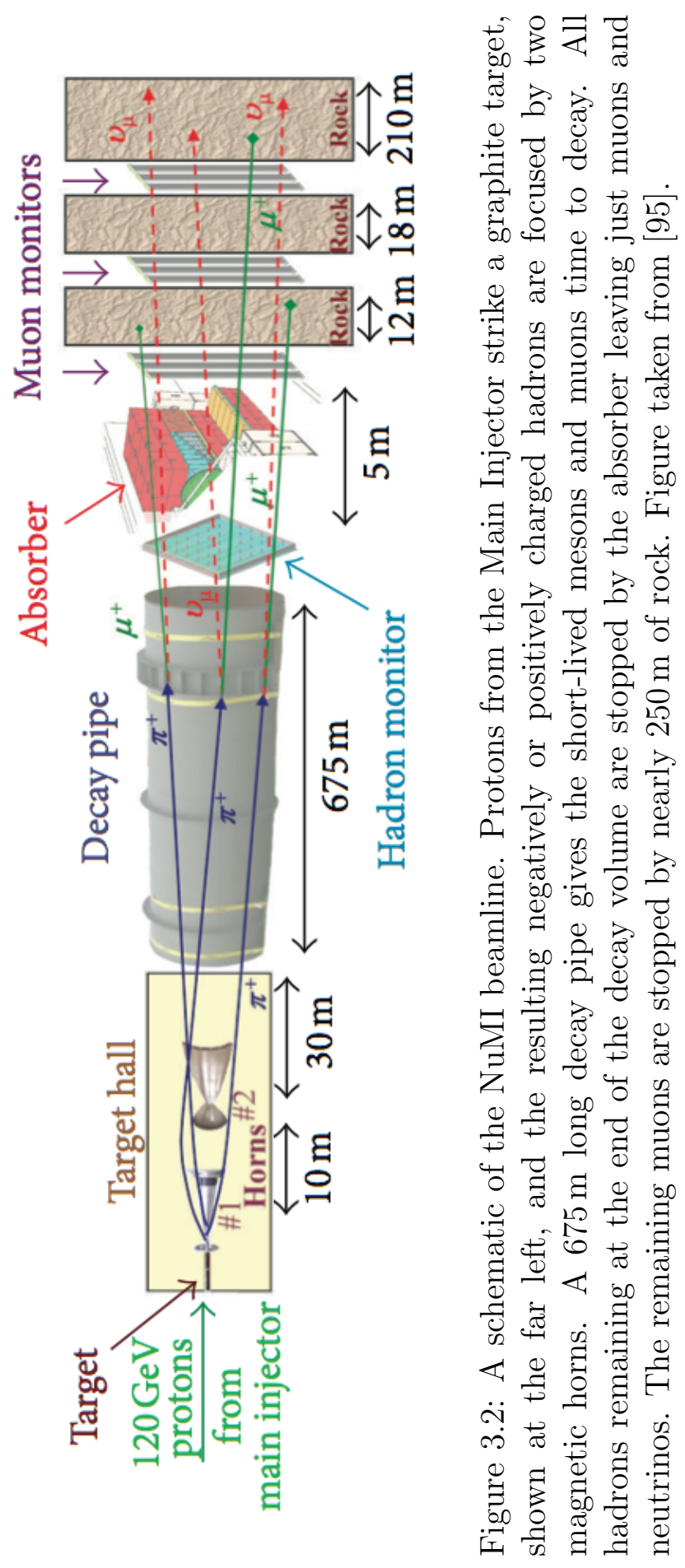




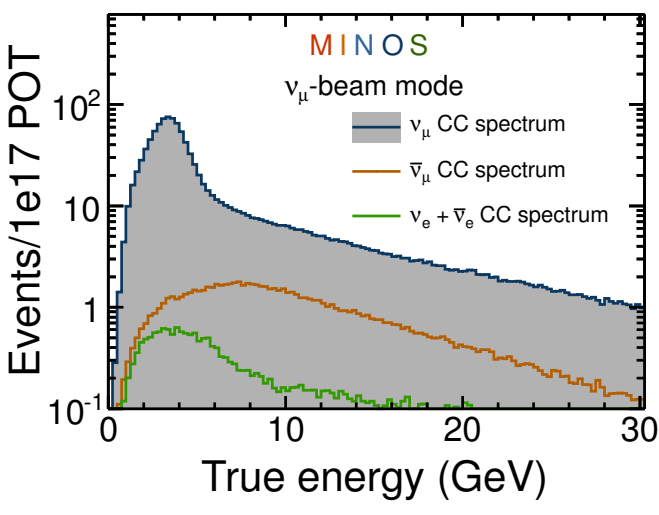

(a) $\nu_{\mu}$-beam mode

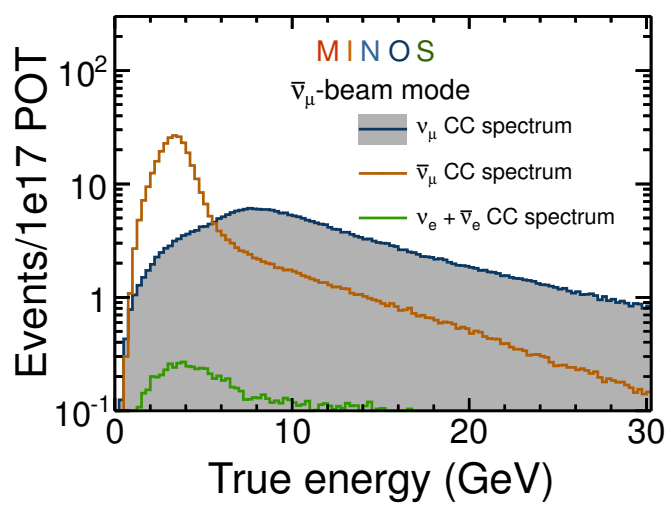

(b) $\bar{\nu}_{\mu}$-beam mode

Figure 3.3: The energy spectra of neutrino components in two modes produced by the NuMI beam: $\nu_{\mu}$-beam mode (left) and $\bar{\nu}_{\mu}$-beam mode (right). The orange lines represent the $\nu_{\mu}$-CC events, the dark blue ones represent the $\bar{\nu}_{\mu^{-}}$ $\mathrm{CC}$ events, and the brighter blue ones present the $\nu_{e}$ and $\bar{\nu}_{e}$ events.

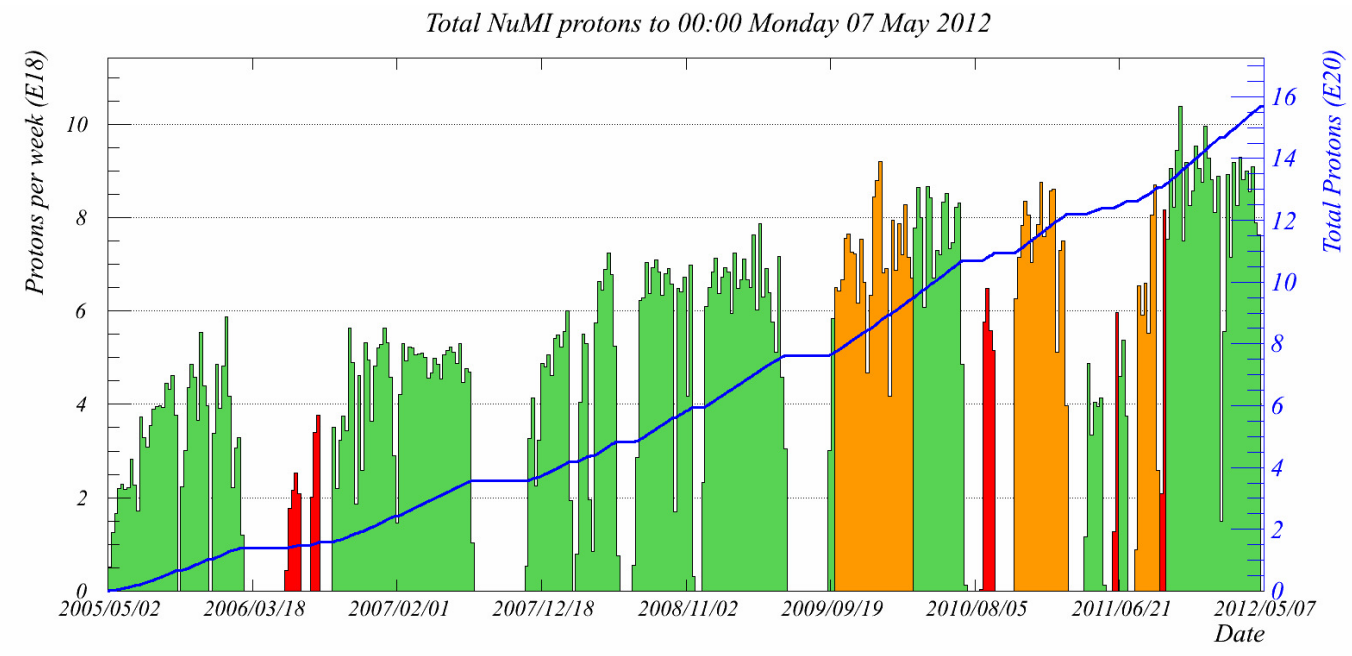

Figure 3.4: The MINOS data accumulated an exposure of $15.6 \times 10^{20}$ protonson-target between 2005 and 2012. Most of this data is in the low energy $\nu_{\mu}$-beam mode (in green) and in the $\bar{\nu}_{\mu}$-beam mode (in orange). The special runs with the higher energy mode or horn off are shown in red. 
The MINOS experiment started to collect data in 2005 and finished in April 2012. For eight years of operation, a total exposure of $15.6 \times 10^{20}$ protonson-target (POT) was delivered to the two MINOS detectors. Figure 3.4 shows the timeline of the NuMI beam exposure delivered to the MINOS detectors.

\begin{tabular}{|c|c|c|c|}
\hline Run & Configuration & Horn polarity & Good data POT $\left(\times 10^{18}\right)$ \\
\hline 1 & LE & Forward & 126.93 \\
\hline 1 & $\mathrm{pHE}$ & Forward & 15.31 \\
\hline 2 & $\mathrm{LE}$ & Forward & 194.27 \\
\hline 3 & LE & Forward & 388.71 \\
\hline 4 & $\mathrm{LE}$ & Forward & 8.84 \\
\hline 4 & LE & Reverse & 170.85 \\
\hline 5 & $\mathrm{LE}$ & Forward & 45.89 \\
\hline 6 & $\mathrm{LE}$ & Forward & 61.62 \\
\hline 7 & LE & Reverse & 124.08 \\
\hline 8 & $\mathrm{LE}$ & Forward & 12.58 \\
\hline 9 & LE & Reverse & 40.80 \\
\hline 10 & $\mathrm{LE}$ & Forward & 238.31 \\
\hline \multicolumn{3}{|c|}{ Total good physics data } & 1428.19 \\
\hline \multicolumn{3}{|c|}{$\begin{array}{l}\text { Total analyzed } \nu_{\mu} \text { beam } \\
\text { Run periods }(1,2,3,5,6,10)\end{array}$} & 1071.04 \\
\hline \multicolumn{3}{|c|}{$\begin{array}{l}\text { Total analyzed } \bar{\nu}_{\mu} \text { beam } \\
\text { Run periods }(4,7,9)\end{array}$} & 335.73 \\
\hline
\end{tabular}

Table 3.1: Summary of the Far Detector collected data in term of the POT exposure. Abbreviation: LE, low energy; pHE, pseudo high energy. 
Table 3.1 shows the summary of the Far Detector collected data. About 10.71× $10^{20}$ POT out of this exposure has been taken with the $\nu_{\mu}$-beam mode and $3.36 \times 10^{20}$ POT taken with the $\bar{\nu}_{\mu}$-beam mode. The remaining subtle portion of data was accumulated in special run periods, which were either with a higher neutrino energy or with the horn off. The special runs are necessary for understanding the detector responses and the neutrino flux. The components of the NuMI facility are briefly described in the following sections:

\section{Target}

The NuMI uses a carbon target in order to maximize $\pi^{ \pm} / K^{ \pm}$production, which consequently maximizes the $\nu_{\mu}$-CC event rate in the MINOS detectors. The target is designed to be sufficiently long enough to have most of the primary protons interacting, but thin enough so that the secondary interactions of the $\pi^{ \pm} / K^{ \pm}$are minimized and energy absorption is small to the extent possible. However, the target should not be too thin since it needs to survive the undesirable target stress resulting from the heat load due to the high intensity proton beam. The target body used by the NuMI, consists of 47 graphite plates of $2 \mathrm{~cm}$ (length) x $0.64 \mathrm{~cm}$ (wide) x $18 \mathrm{~cm}$ (tall). Graphite can sustain very high temperatures and is relatively strong, enabling it to survive bombardment from the intense proton beam. Also, a pair of stainless steel tubes circulates chilled water, decreasing the heat generated by the proton collisions and continuously cooling down the interaction target. It took seven NuMI targets, summarized in Table 3.2, to complete entire MINOS run period. 


\begin{tabular}{|c|c|c|c|c|}
\hline Target & Horn & $\begin{array}{r}\text { Distance from } \\
\text { horn } 1(\mathrm{~cm}) \\
\end{array}$ & Configuration & Start - end date \\
\hline NT-01 & PH1-01 & $99.59+/-0.27$ & LE100 200kA FHC & $05 / 01 / 2005-05 / 12 / 2005$ \\
\hline NT-01 & PH1-01 & $249.59+/-0.27$ & LE250 200kA FHC & $05 / 12 / 2005-05 / 20 / 2005$ \\
\hline NT-01 & PH1-01 & $9.59+/-0.27$ & LE10 185kA FHC & $05 / 12 / 2005-05 / 20 / 2005$ \\
\hline NT-01 & PH1-01 & $149.59+/-0.33$ & LE150 200kA FHC & $05 / 18 / 2006-06 / 11 / 2006$ \\
\hline NT-01 & PH1-01 & $249.59+/-0.27$ & LE250 185kA FHC & $06 / 11 / 2006-08 / 14 / 2006$ \\
\hline NT-02 & PH1-02 & $8.34+/-0.35$ & LE10 185kA FHC & $07 / 12 / 2008-06 / 13 / 2009$ \\
\hline NT-03 & PH1-02 & & $\begin{array}{l}\text { several locations for } \\
\text { muon monitor tests }\end{array}$ & 09/11/2009 - 09/15/2009 \\
\hline NT-03 & PH1-02 & $9.4+/-0.2$ & LE10 & 09/15/2009- 07/13/2010 \\
\hline NT-04 & PH1-02 & $99.57+/-0.22$ & LE100 200kA FHC & $08 / 22 / 2010-09 / 03 / 2010$ \\
\hline NT-04 & PH1-02 & $99.57+/-0.22$ & LE100 200kA RHC & $09 / 03 / 2010-09 / 08 / 2010$ \\
\hline NT-04 & PH1-02 & $249.57+/-0.22$ & LE250 200kA FHC & $09 / 08 / 2010-09 / 17 / 2010$ \\
\hline NT-04 & PH1-02 & $249.57+/-0.22$ & LE250 200kA RHC & $09 / 17 / 2010-10 / 31 / 2010$ \\
\hline NT-05 & PH1-02 & $8.85+/-0.22$ & LE10 185kA RHC & $10 / 31 / 2010-02 / 24 / 2011$ \\
\hline NT-06 & PH1-02 & $9.18+/-0.22$ & LE10 185kA FHC & $04 / 09 / 2011-04 / 18 / 2011$ \\
\hline NT-06 & PH1-02 & $9.18+/-0.22$ & horn off test LE10 & $04 / 18 / 2011-05 / 02 / 2011$ \\
\hline NT-06 & PH1-02 & $9.18+/-0.22$ & LE10 185kA FHC & 05/02/2011 - 05/16/2011 \\
\hline NT-01 & PH1-02 & $250.09+/-0.22$ & LE250 200kA FHC & $06 / 10 / 2011-06 / 21 / 2011$ \\
\hline NT-01 & PH1-02 & $10.40+/-0.22$ & LE10 185kA FHC & $06 / 21 / 2011-07 / 08 / 2011$ \\
\hline NT-01 & PH1-02 & $9.18+/-0.29$ & LE10 185kA RHC & $07 / 21 / 2011-09 / 15 / 2011$ \\
\hline NT-07 & PH1-02 & $99.17+/-0.29$ & LE100 200kA RHC & $09 / 24 / 2011-10 / 05 / 2011$ \\
\hline NT-07 & PH1-02 & $9.17+/-0.29$ & LE10 185kA FHC & $10 / 06 / 2011-03 / 14 / 2012$ \\
\hline
\end{tabular}

Table 3.2: Summary of target location along the beam-line for entire MINOS run period. 


\section{Focusing horns}

Focusing the $\pi^{ \pm} / K^{ \pm}$produced from the target is the essential technique for making an intense neutrino beam. For pion decays, at a given angle $\theta_{\pi}$ with respect to the pion direction, the neutrino flux is calculated by:

$$
\phi_{\nu} \propto \frac{1}{4 \pi z^{2}}\left(\frac{2 \gamma}{1+\gamma^{2} \theta_{\pi}^{2}}\right)^{2},
$$

where $z$ is the distance from the observed point to the pion decay point, and $\gamma$ is the Lorentz factor of pion. Without focusing [75]:

$$
\theta_{\pi} \approx \frac{p_{T}}{p_{\pi}} \approx \frac{2}{\gamma}
$$

This implies that the perfect focusing of pions, in principle, would increase the neutrino flux by a factor of 25 .

The idea of using the magnetic horns for focusing mesons was proposed by Simon van der Meer [96] in 1961. The NuMI focusing is operated by a set of two horns of pulsed transmission-line magnets, which creates a focusing toroidal magnetic field. Since the focal length of the horn is proportional to the particle's momentum, it allows one to focus particles in a desired momentum range over a wide range of production angles. By adjusting the locations of

the second horn and of the target with respect to the first horn, mesons of specific energy range are selected. This allows the NuMI to produce different neutrino energy beams with the same energy proton source. 


\section{Decay tunnel}

The decay volume is an evacuated, encased in concrete, and watercooled steel tube of $1 \mathrm{~m}$ (radius) x $675 \mathrm{~m}$ (length), pointing towards the Soudan Underground Laboratory, MN. While propagating through the decay pipe, a fraction of mesons decay following Eq. (3.1) and result in a forward-going neutrino beam. The neutrino energies and the beam flux are derived from the parent mesons by:

$$
\begin{aligned}
& E_{\nu}=\frac{\left(1-m_{\mu}^{2} / m_{(\pi, K)}^{2}\right) E_{(\pi, K)}}{1+\gamma^{2} \theta^{2}} \\
& \Phi\left(\nu_{\mu}\right)=\left(\frac{2 \gamma}{1+\gamma^{2} \theta^{2}}\right)^{2} \frac{A}{4 \pi r^{2}}
\end{aligned}
$$

where $\gamma$ is the Lorentz factor of pions, $\theta$ is the angle between parent mesons and directions of resultant neutrinos, $A$ is the cross-sectional area of the detector, and $r$ is the distance from the neutrino source to the detector. The energy and the flux of the neutrinos peak along the meson flight direction, $\theta=0$. For well-focused mesons and a detector on the axis of the pion beam, i.e $\theta \approx 0$, the energy of neutrinos is around $43 \%$ (96\%) of their parent pion (kaon) energy.

\section{Hadron absorber}

The hadron absorber is located at the back end of the NuMI decay tunnel. Its aim is to absorb the hadrons that are produced in the target and decay pipe. Steel and concrete are used to make the absorber. The absorber also has a water-cooled aluminum central core, which helps to remove the heat generated by the protons and secondary hadrons. 


\section{Muon monitors}

There are three muon monitors located downstream of the hadron absorber as depicted in Figure 3.2. Each muon monitor comprises a $9 \times 9$ array of $10.2 \times 10.2 \mathrm{~m}^{2}$ ionization champers distributed uniformly inside 9 tubes [97]. The motivation for the installation of muon monitors is to measure the muon flux produced from the decay of pions and kaons $\left(\pi / K \rightarrow \mu \nu_{\mu}\right)$. The measurement sets constrain the momentum distribution of the parent pions and kaons, which consequently infer with the neutrino flux.

\subsection{Detector technology}

The MINOS experiment consists of two magnetized spectrometers separated by a baseline of $734 \mathrm{~km}$. These two detectors, made of an iron and plastic scintillator, are hadronic sampling and muon tracking calorimeters designed to measure the energy of events coming from the NuMI neutrino beam.

\subsubsection{Detector overview}

MINOS uses the two functionally similar detectors to measure the neutrino energy spectra before (at the Near Detector) and after (at the Far Detector) oscillations. The two-detector technique helps to substantially reduce the systematic uncertainties of the neutrino flux, the cross-sections and the detector responses.

To precisely measure the neutrino oscillation parameters, we need to identify neutrino events and reconstruct their energy with high resolution. In 
order to achieve these goals, tracking and sampling calorimeters have been constructed in both detectors. These calorimeters are segmented into the interleaved planes of a magnetized steel and plastic scintillator. While the magnetized steel planes function as the targets of neutrino interactions, the scintillator planes, composed of $4.1 \mathrm{~cm}$ wide, $1.0 \mathrm{~cm}$ thick plastic scintillator strips, are active regions of the detectors downstream of these targets.

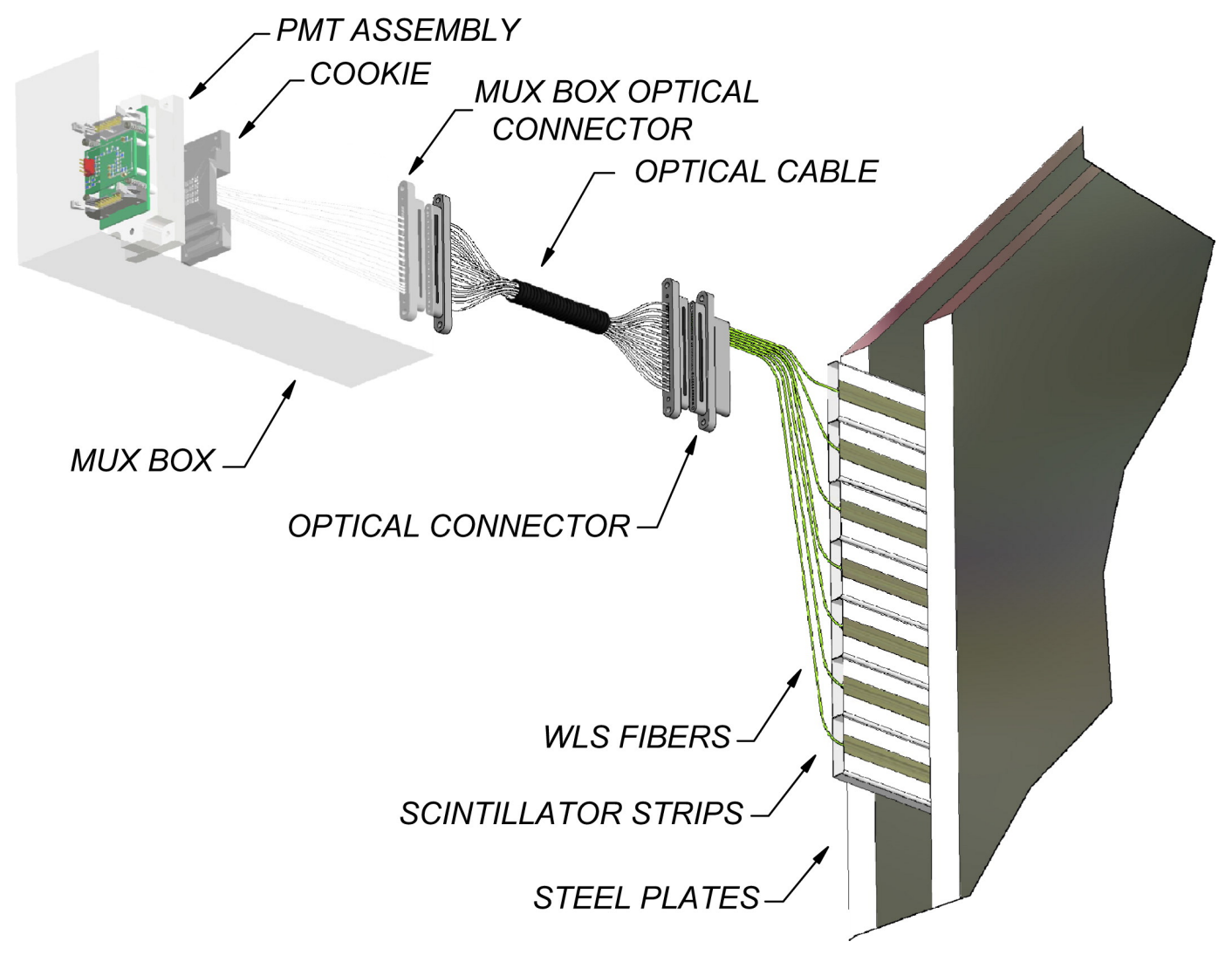

Figure 3.5: Basic elements of optical readout using in the MINOS detectors. The scintillator lights are reflected in scintillators until collected by the wavelength shifting (WLS) fibers, then transferred to the PMT via optical cables. Figure taken from [98]. 
Figure 3.5 shows the schematics of the optical readout in the MINOS detectors. Neutrinos interact with the steel to produce a number of secondary particles. The resulting charged particles, typically muons and pions, generate small amounts of light when passing through the scintillators. The scintillation light, proportional to the deposited energy, is then collected by optical fibers and amplified by photo-multiplier tubes (PMT) before being read by an electronic data acquisition system. The topology and timing information of the deposited hits are exploited to reconstruct neutrino events, which give us the footprints of the neutrino interaction types.

The magnetic fields in the detectors bend the trajectories of charged particles and their curvature is utilized to estimate their momentum and their charge-sign. This allows us to distinguish $\mu^{+}$from $\mu^{-}$induced by $\bar{\nu}_{\mu}$ and $\nu_{\mu^{-}}$ CC interactions respectively. Consequently, the $\bar{\nu}_{\mu}$ disappearance is observed directly and the antineutrino oscillation parameters are measured independently. This enables MINOS to test the CPT symmetry in the lepton sector. The following are brief descriptions of the MINOS detectors:

\section{Near Detector}

This detector is located $100 \mathrm{~m}$ underground (225 meters-water-equivalent m.w.e) at Fermilab, $1.04 \mathrm{~km}$ downstream from the NuMI target. Figure 3.6 shows the schematic drawing and the real image of the Near Detector. The NuMI neutrino beam reaches the Near Detector with a diameter of $50 \mathrm{~cm}$. The detector is composed of 282 planes of $2.54 \mathrm{~cm}($ thick $) \times 3.8 \mathrm{~m}($ high $) \times$ 
$4.8 \mathrm{~m}$ (wide) steel squashed-octagon planes and 153 scintillator planes, which are made of $4.1 \mathrm{~cm}$ (wide) $\times 1.0 \mathrm{~cm}$ (thick) plastic scintillator strips. Each plane has a hole of $30 \mathrm{~cm} \times 30 \mathrm{~cm}, 59 \mathrm{~cm}$ from the center where the magnetic coil resides. The overall mass of the Near Detector is 980 tons .

The detector is functionally divided into four longitudinal regions [90]: (i) veto region (planes 1-20) used to shield the upstream neutrinos, (ii) target region (planes 21-60) to provide the fiducial volume for selecting neutrino interaction events, (iii) calorimeter region (planes 61-120) used to measure event topologies, electromagnetic and hadronic shower energy, and muon momentum, (iv) spectrometer region (planes 121-281) used to identify muons and measure the energy of muon tracks.

\section{Far Detector}

This detector is in the Soudan mine, $735.34 \mathrm{~km}$ downstream of the NuMI target. The schematic drawing and the real image of the Far Detector is shown in Figure 3.7. The detector is situated at a depth of $705 \mathrm{~m}$ (2070 m.w.e), which aids for cosmic ray shielding. The NuMI neutrino beam reaches the Far Detector with a diameter of $1 \mathrm{~km}$. This reduces the beam intensity by a factor of $\sim 10^{5}$ relative to the Near Detector. The Far Detector is composed of 486 planes of 2.54 thick steel measuring $8 \mathrm{~m} \times 8 \mathrm{~m}$ and 484 scintillator planes. This gives it an overall mass of 5.4 ktons. The detector consists of two super modules of 249 and 237 planes, each separated by a gap of $1.1 \mathrm{~m}$. 


\section{Calibrator Detector}

For studying the calorimetric response of the Near and Far detectors, MINOS built a prototype, named the Calibration Detector. This detector helps to determine the absolute and relative energy responses of the Near and Far detectors, which play a crucial role for event energy reconstruction. The detector consists of 60 planes of $2.5 \mathrm{~cm}$ (thick) x $1 \mathrm{~m}$ x $1 \mathrm{~m}$ steel planes. During 2001-2003, this detector was exposed to a 0.2 - $10 \mathrm{GeV}$ energy range beam of

$p, \pi^{ \pm}, \mu^{ \pm}$, and $e^{ \pm}$particles at the CERN Proton Synchrotron accelerator in Geneva. 


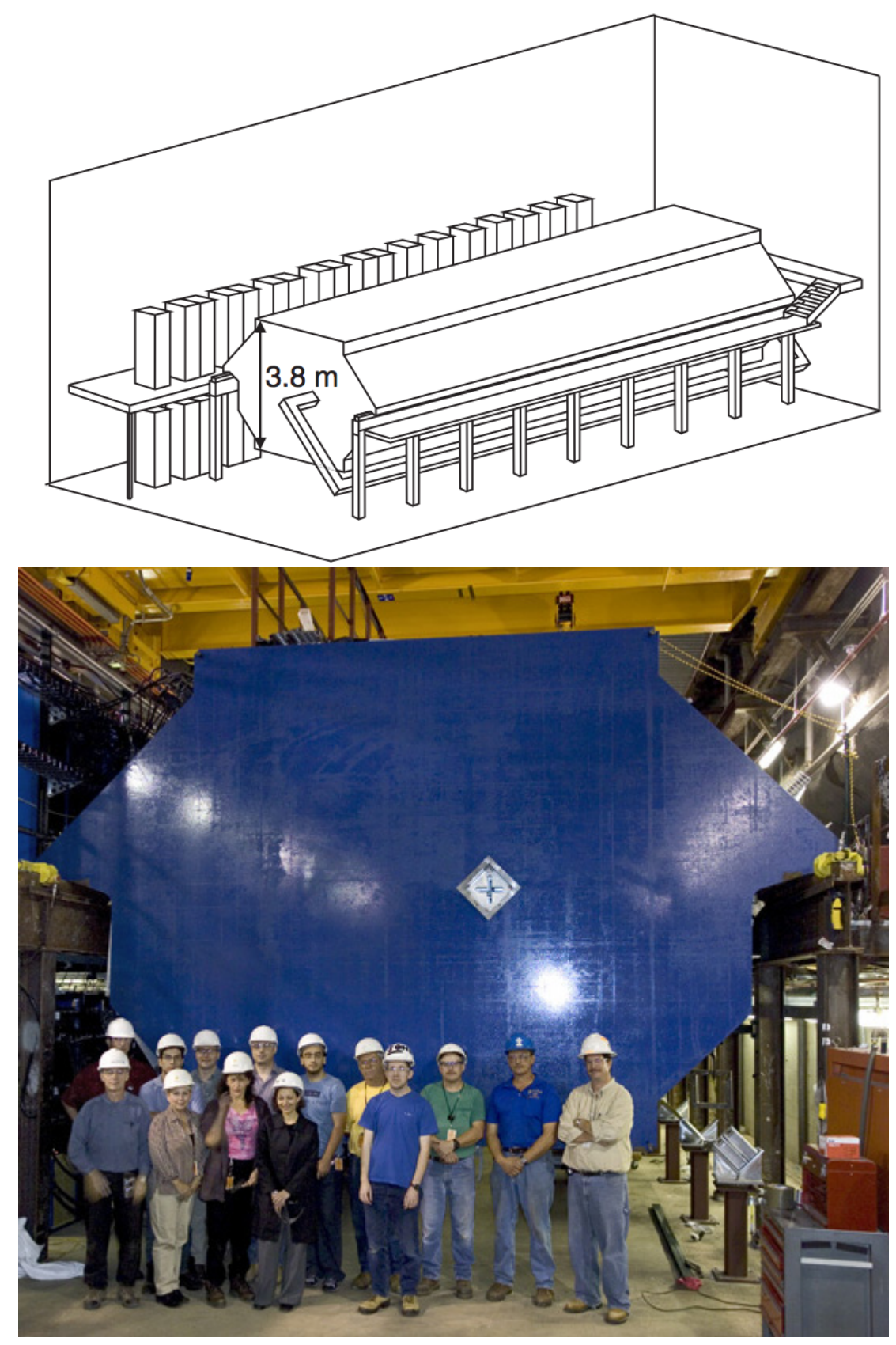

Figure 3.6: The Near Detector with the schematic drawing in the top and the real one in the bottom. Figure taken from [48]. 


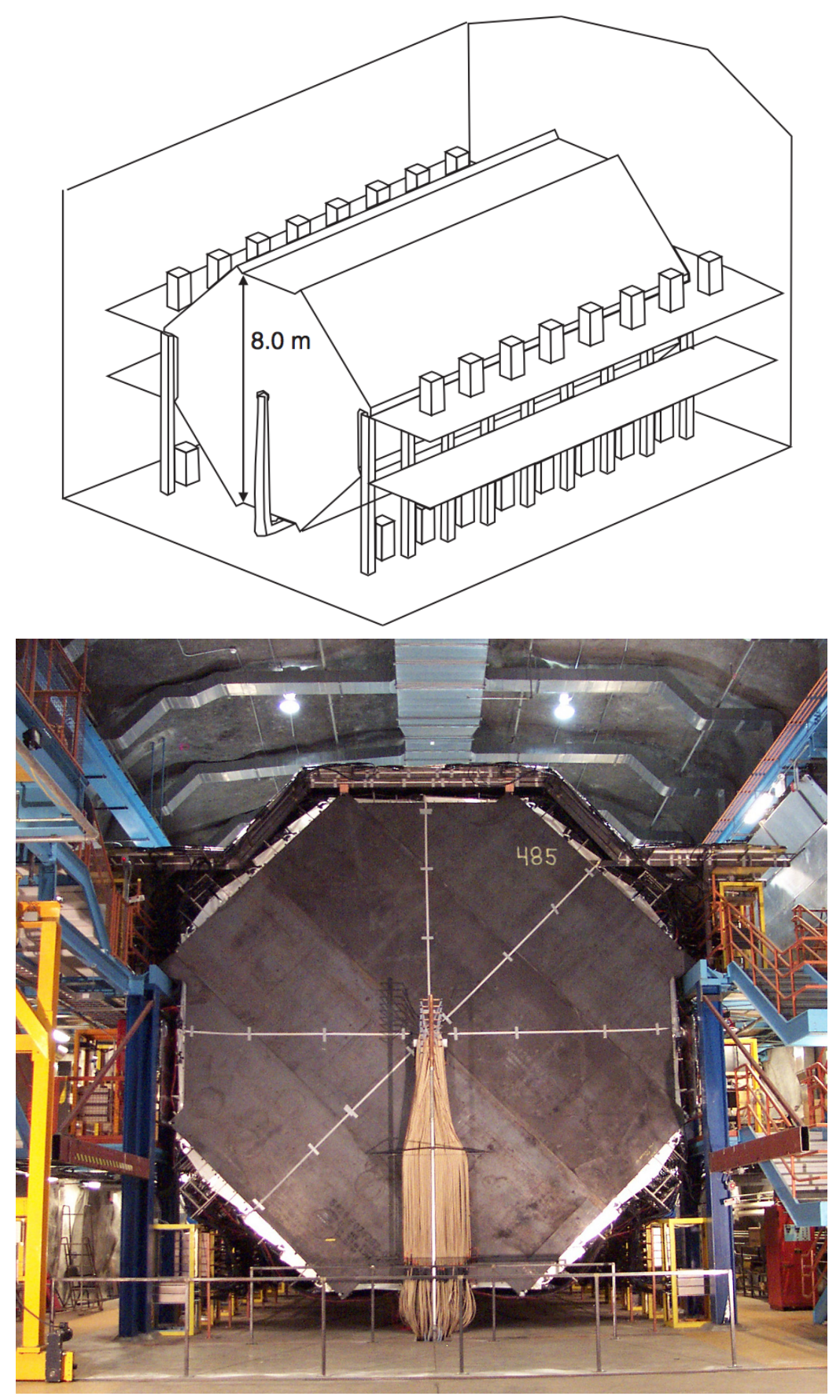

Figure 3.7: The Far Detector with the schematic drawing in the top and the real one in the bottom. Figure taken from [48]. 


\subsubsection{Steel and magnetization}

The MINOS detectors use steel planes as the targets for nuclear interactions, the passive absorbers for resultant particles, and the mechanical support for the scintillator planes. In order to give it high tensile strength and have good magnetic properties as well, the carbon content in the steel was chosen to be between $0.04-0.06 \%$ (by weight), consistent with $\mathrm{AISI}^{3} 1006$ low-carbon steel. Furthermore, to mitigate the systematics between the two detectors, the steel materials are required to have similar densities and magnetic properties in both detectors. The measured thickness of steel planes is $2.563 \pm 0.002 \mathrm{~cm}$ at the Near Detector and $2.558 \pm 0.005 \mathrm{~cm}$ at the Far Detector. Their density is recorded as $7.85 \pm 0.03 \mathrm{~g} / \mathrm{cm}^{3}$ with no systematics contributed by the density differences between the Near and Far detectors.

The MINOS magnetization is designed to measure the muon momentum $(\mathrm{P})$ via its curvature with resolution of $\sigma_{P} / P \sim 12 \%$. The coil hole systems that produce the magnetic field in the detectors are independently constructed to take into account the differences of detector geometries and laboratory infrastructures at the two sites. The coil hole at the Near Detector applies a $40 \mathrm{kA}$ turn current that produces an average magnetic field of 1.17 $\mathrm{T}$ in the detector. Two coil holes are used in the Far Detector in order to magnetize the two supermodules independently. A $15.2 \mathrm{kA}$ turn total current is operated to provide an average $1.27 \mathrm{~T}$ magnetic field strength in the Far Detector.

\footnotetext{
${ }^{3}$ American Iron and Steel Institute
} 


\subsubsection{Scintillator and module design}

The extruded plastic scintillators are used as the active detector elements for producing the scintillation light from energy deposited by the charged particles. MINOS uses polystyrene scintillator strips of $4.1 \mathrm{~cm}$ (wide) $\times 1.0 \mathrm{~cm}$ (thick) co-extruded with a layer of $\mathrm{TiO}_{2}$ doped polystyrene to provide a reflective boundary.
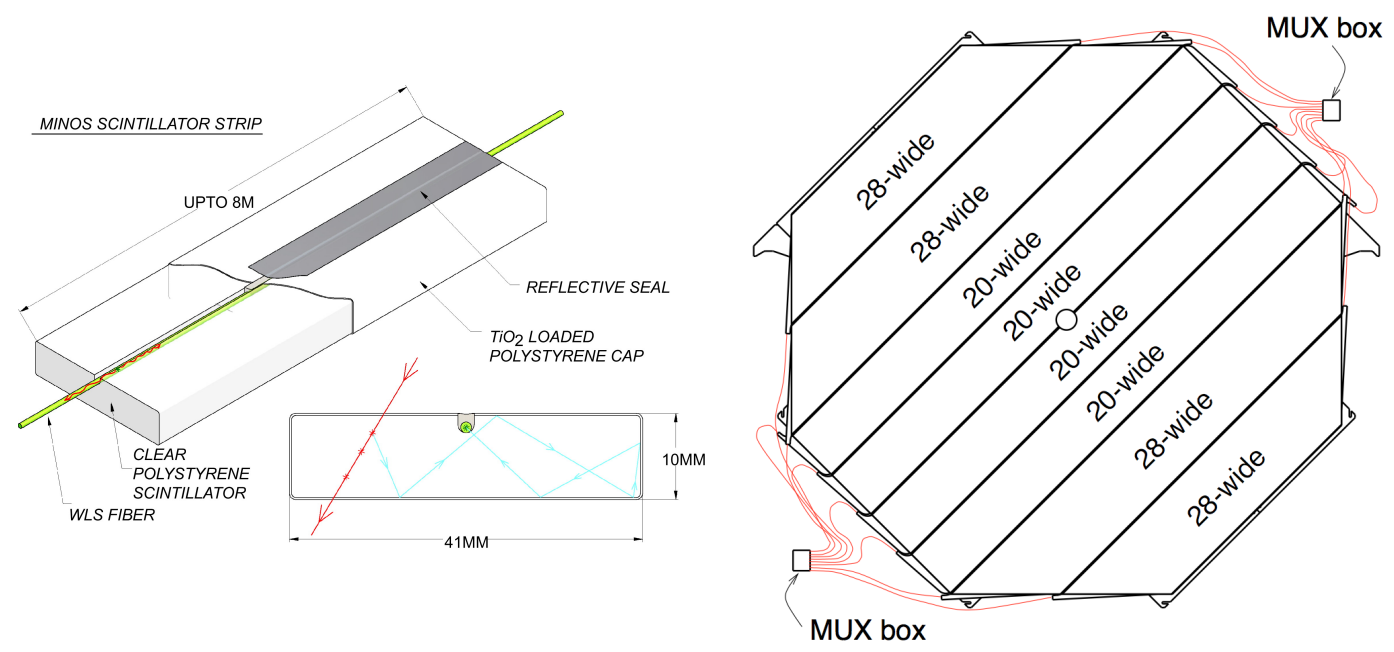

Figure 3.8: Cutaway drawing of a single scintillator strip (left): light is produced by ionizing particle, reflected inside the strip before captured by the WLS fibers, guided to the edge of detector and routed to the PMTs. The schematic design of scintillator module is shown on the right.

Either 20 strips or 28 strips are glued to form a scintillator module. The lengths of scintillator strips are adjusted depending on their positions on the module. Along each strip, a $1.0 \mathrm{~mm}$ (wide) $\times 2.0 \mathrm{~mm}$ (deep) groove is made to embed a $1.2 \mathrm{~mm}$ diameter wavelength shifting (WLS) fiber. Figure 3.8 shows 
the cutaway drawing of a single scintillator strip and the schematic structure of the scintillator module. In the Far Detector, the WLS fibers are read out from both ends, while the shorter Near Detector fibers are read out only from one end. These fibers absorb light in the blue part of the spectrum, which peaks at $420 \mathrm{~nm}$, is then re-emitted in the green part of the spectrum, which peaks at $520 \mathrm{~nm}$.

The scintillator modules are mounted on the steel planes at a $45^{\circ}$ angle with respect to the horizontal line. Successive planes are installed at rightangles to each other, allowing events to be observed in " $U=\frac{1}{\sqrt{2}}(x+y)$ " and " $V=\frac{1}{\sqrt{2}}(x-y) "$ views, as shown in Figure 3.9. The high spatial resolution from these two views facilitates reconstructing event topology in three dimensions.
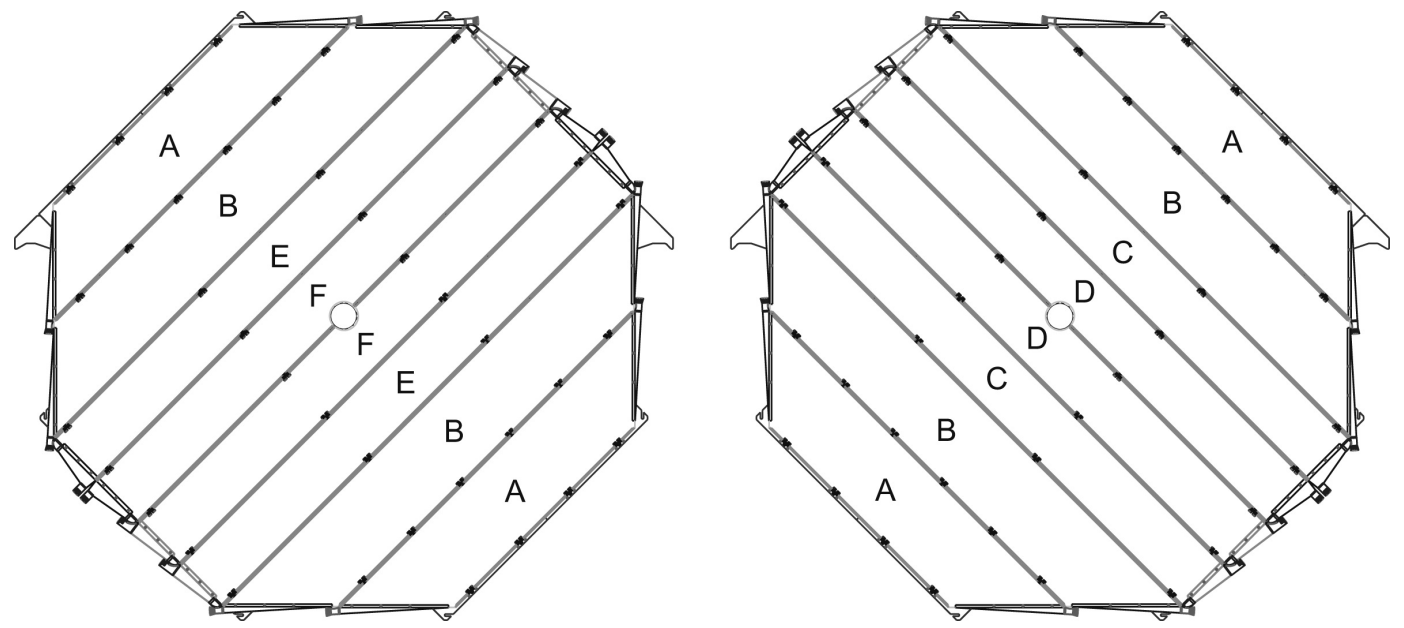

Figure 3.9: Layout of $\mathrm{U}$ (left) and V (right) modules on the Far Detector planes. U- and V-type planes are interleaved. A and B module types have 28 scintillator strips and the other types have 20 strips. Figure taken from [98]. 


\subsubsection{PMT and enclosures}

The scintillator light is shifted and guided to the edge of the detectors by the WLS fiber before being carried by clear fiber ribbons to the multiplexing boxes where the PMTs are mounted. A multiplexing scheme is employed to reduce the number of PMTs needed for the front-end electronics. At the Far Detector, the multiplexing groups eight strip ends which are separated by about $1 \mathrm{~m}$ and read by one pixel of the Hamamatsu M16 PMT, depicted in Figure 3.10.

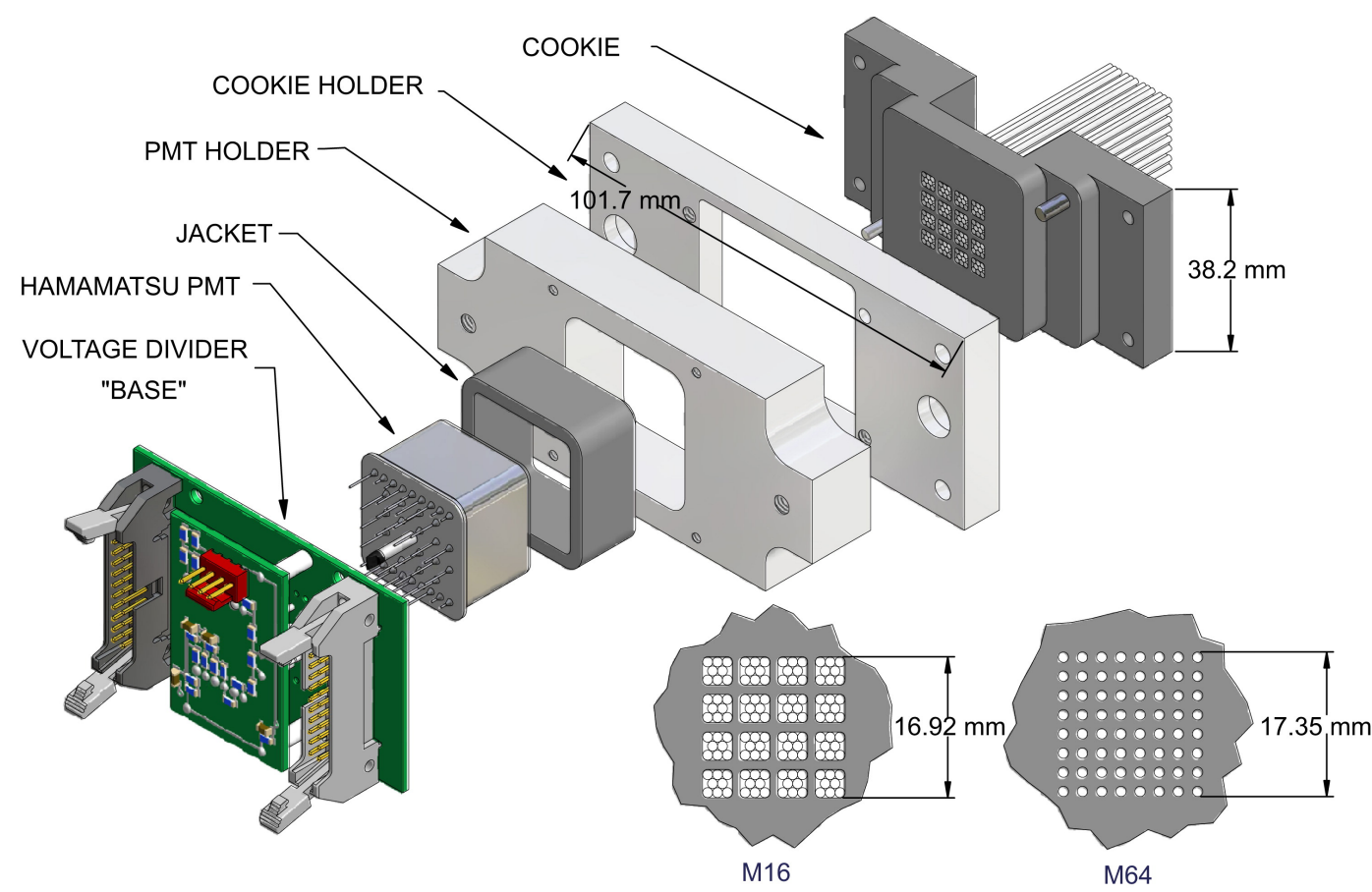

Figure 3.10: Mounting assembly of Hamamatsu M16 PMT. The M64 "cookie" layout is shown on the lower right of the figure. Figure taken from [98]. 
Totally, 1452 PMTs are needed for 185,856 strip ends. At the Near Detector, strips in the calorimeter region are read out individually while a multiplexing scheme of four $1 \mathrm{~m}$-separated strips is employed in the spectrometer region. The $1 \mathrm{~m}$ separation guarantees the effectiveness of the demultiplexing algorithm. To keep the same light yield between the two detectors, strips at the Near Detector are read out at one end by the M64; while strips at the Far Detector are read out at both ends by the M16. The nominal operating voltage for both types of PMTs is $\sim 800 \mathrm{~V}$, allowing a maximum gain of $0.8 \times 10^{6}$ with the M64 and $1.0 \times 10^{6}$ with the M16. All PMTs have clear fibers and cable connectors housed in the light-tight steel enclosures. This design is chosen to minimize the crosstalk between the PMTs. At the Near Detector, each M64 resides in an individual closure while at the Far Detector, three M16 are mounted into one closure, called a multiplexer (MUX) box.

\subsubsection{Electronics and DAQ}

The main goals of the electronics are: to provide adequate information for separating the $\mathrm{NC}$ and $\mathrm{CC}$ neutrino interactions and to measure the energy of neutrino events with high resolution. The difference of event rates require distinct electronic systems in the two detectors.

\section{Near Detector}

The instantaneous event rate is much greater in the Near Detector than in the Far Detector. This requires very fast digitized electronics with 
no dead time during the $10 \mu s$ beam spill window. The Near Detector uses a Charge Integration Encoder $(\mathrm{QIE})^{4}$ to digitize continuously the signal from PMT pixels at the $53.103 \mathrm{MHz}(\sim 18.83 \mathrm{~ns}) \mathrm{RF}$ frequency of the Main Injector.

\section{Far Detector}

The electronics are designed specifically to work with the low rate at Soudan mine, $0.5 \mathrm{~Hz}$ for both cosmic muons and beam muons. The signal rate is dominated by the detector noises, which are around $3-6 \mathrm{kHz}$ per PMT. Thus, the commercial 14-bit $10 \mathrm{MHz}$ Analog-to-digital converters (ADCs) are adequate to operate at the Far Detector with very low dead time.

The operation of the data acquisition systems (DAQ) at the two detectors are functionally identical. Their job is to read out the raw data from the front-end electronics and transfer them to the farm of computers where the software selects interesting events for monitoring and calibration.

\subsection{Signal calibration}

The recorded signals in the MINOS detectors must be calibrated to correct for variations in the response of scintillator strips and readout channels. For each physics event, the detectors record a series of raw hits with information of position, timing and pulse height. From these raw hits, to obtain the event energy, a series of multiplicative factors is implemented to convert the raw photomultiplier $Q_{\text {raw }}(i, t, x)$ recorded in channel $i$ at the time $t$ at position

\footnotetext{
${ }^{4}$ Custom integrated circuit designed at Fermilab
} 
$x$ into a calibrated signal $Q_{\text {cal }}[98]$ :

$$
Q_{\text {cal }}=Q_{\text {raw }}(i, t, x) \times D(t) \times L\left(i, Q_{\text {raw }}\right) \times U(i, t) \times A(i, x) \times E,
$$

where:

- $D(t)$ : corrects for the drifts in a channel response. This factor is computed using the overall detector responses to through-going cosmic ray muons. This is caused by scintillator and fiber aging and consists of drift of the entire optical system, drifts of PMT gains and electronics response. The detector responses vary with time at a level of a few percent.

- $L\left(i, Q_{\text {raw }}\right)$ : corrects for the non-linear channel responses. This is due to the 5-10\% non-linearity of PMT responses at light levels of approximately 100 photoelectrons. This factor is estimated using a Light Injection system, which uses LED lights to create fake input signals. Comparisons are made between the detector outputs to get an independent measurement of light intensity.

- $U(i, t)$ : corrects for the non-uniform channel-to-channel responses. This factor is taken into account by the fact that the responses of strips fluctuate due to the variation in scintillator light yield, light collection efficiency of the WLS fibers, attenuation of the clear fibers, and quantum efficiency of the PMTs. A variation of $30 \%$ of the individual strip response is found by using through-going cosmic ray muons for calibration. 
- $A(i, x)$ : corrects for the WLS fiber attenuation. This factor is estimated by using a test-stand and results from fitting the data to a double exponential:

$$
A(i, x)=A_{1}(i) e^{-x / L_{1}}+A_{2}(i) e^{-x / L_{2}}
$$

where $x$ is the length along the strip and $L_{1}, L_{2}$ are two attenuation lengths. The attenuation corrections vary by approximately $50 \%$ for a $3 \mathrm{~m}$ scintillator strips in the Near Detector and $30 \%$ for an $8 \mathrm{~m}$ scintillator strips in the Far Detector.

- $E$ : is the overall scale factor that converts the fully corrected signal to an absolute energy unit for the two detectors. This factor, called Muon Energy Unit (MEU), is computed by using the stopped cosmic ray muons in the detectors. To reduce the uncertainty of MEU, only segments of muon tracks with small $d E / d x$ variation is chosen.
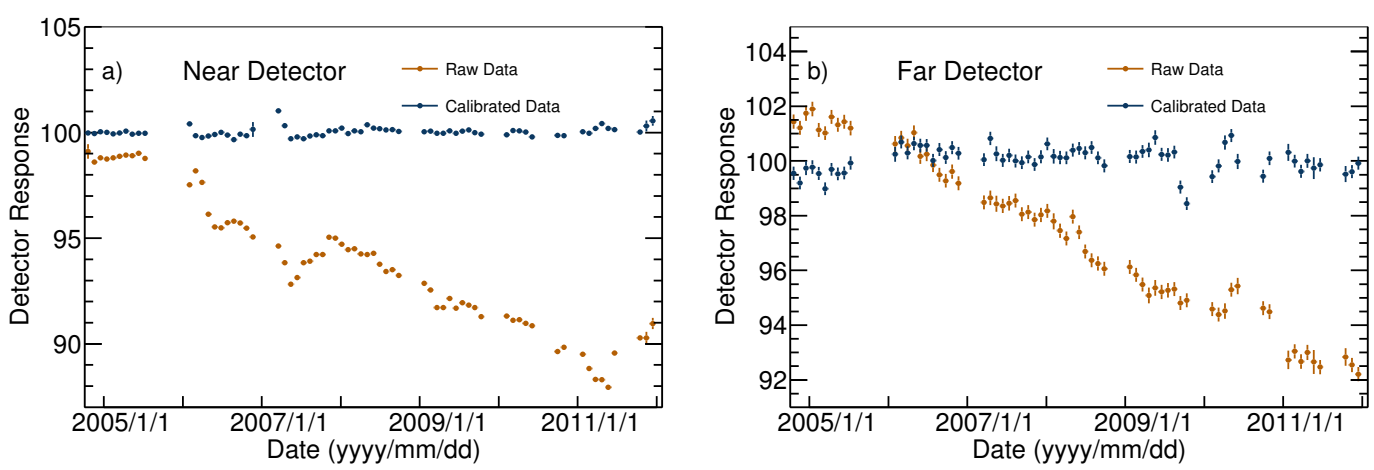

Figure 3.11: The response in MEU for the Near Detector (left) and Far Detector (right) as the function of time. The MEU values are stable over time to within $0.5 \%$ for the Near Detector and $1.5 \%$ for the Far Detector. 
The calibration chain results in the correction of response variations for the two detectors. Figure 3.12 shows an example of calibration result on the Far Detector response. After applying the corrections, the spatial variation in detector response is estimated to be within 1\%. Figure 3.11 shows the responses in MEU as the function of time for the two detectors before and after applying calibration corrections. The calibrated MEU values are stable within $0.5 \%$ for the Near Detector and $1.5 \%$ for the Far Detector while the uncalibrated MEU values vary up to $25 \%$.
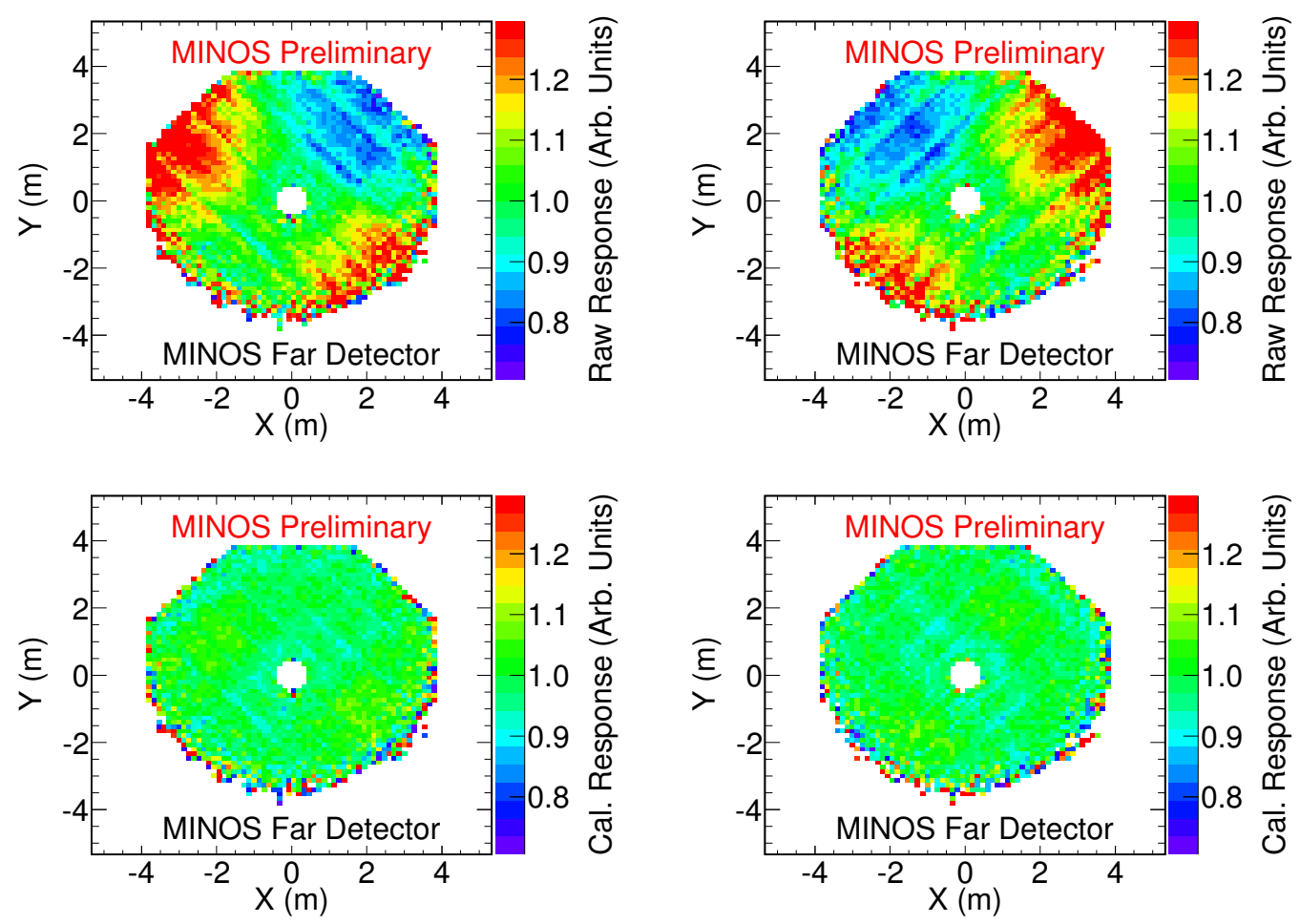

Figure 3.12: The raw response (in ADCs) of the Far Detector as the function of detector position for U strips (left) and V strips (right) before (top) and after (two bottom) calibration. 


\subsection{Monte Carlo simulation}

The chain of MINOS Monte Carlo (MC) processes begins with the simulation of the neutrino flux from the NuMI beam by using the FLUGG program [99]. This program incorporates the geometry modeled by a GEANT3 [100]based GNUMI framework into FLUKA [101], in order to simulate the proton interactions on the target, the propagation and re-interaction of the produced particles and the particle decays in a decay pipe. The output of this step is a collection of neutrinos coordinated at the decay point of their parent particle with their flavor labeled and specific values for energy and momentum. The neutrino flux is simulated separately for each run period, taking into account the shift of target position and the effect of introducing helium into the decay pipe. The next step in the MC chain is simulating the neutrino interactions in each detector. This is done by employing the NEUGEN3 package [102] with MODBYRS-4 cross-section model and the resultant particles are passed to the GEANT3-based detector model to simulate the underlying physics. Additionally, the development of hadronic showers is modeled using the GCALOR package [103]. The lowest level of the MC process is the simulation of the energy deposits, converting them into scintillation light output and translating them into mimicking detector readout signals. This whole chain produces a raw MC sample.

The produced MC raw samples are reconstructed in the same manner as the real data, but true information of the interaction is included. This procedure allows us to understand the efficiency of event reconstruction and 
how well we calibrate the system. Normally, the simulation is not precise since it can not take into account all the detector effects, which have not been seen until long after the data have been taken. Thus retuning the MC sample to force agreement between the data and MC is necessary.

In the Near Detector, the effects of multiple interactions from the same beam spill are taken into account by overlaying the individually simulated events. Effects of neutrino oscillations are taken into account at the analysis level by reweighing each event with its true energy-depended probability. Furthermore, the potential appearance of $\nu_{\tau}$ events requires one to have a tau MC sample, which is normally generated by switching from $\nu_{\mu}$ to $\nu_{\tau}$ in the original neutrino flux.

\subsection{Event reconstruction}

The $\nu_{\mu}$ charged-current events are characterized by a muon track and a number of hadronic showers. What is recorded in the detectors are the raw deposited hits along with the topological and timing information. From these raw hits, an ordered set of algorithms are applied in order to ultimately

find the candidate tracks and showers for each events. The following are the highlights of algorithms employed for the reconstruction process in MINOS:

\section{Digit formation and demultiplexing}

Scintillation light, generated when charged particles hit the scintillators, is converted into a digitized measurement of pulse height, called digit, 
and recorded by the electronics. Each digit is registered with a time-stamp and a list of possible associated strip ends. The Far Detector and the spectrometer region of the Near Detector are multiplexed. A demultiplexing algorithm is used to identify which of eight (Far Detector) or four (Near Detector spectrometer) strip ends are associated with each digit. Since the Far Detector is read out at both ends of the strip, the demultiplexing algorithm finds the digit pair when two digits are identified as originating from the same strip.

\section{Strip formation and slicing}

The strip, formed by grouping digits arrived at the same time from each end of the scintillator strip in the Far Detector, or from the single end in the Near Detector, presents a single energy deposit in a scintillator strip. In the Far Detector, the event rate is low enough so that only one strip is typically formed, while in the Near Detector, multiple strips from a number of neutrino interactions per spill are expected. To simplify the reconstruction process, the activity in the detector in each beam spill is divided into one or more events based on the localization in time-space. The remaining chain of reconstruction is then applied to each slide (i.e., individual event). In this sense, a slice is defined as any collection of strips that are spatially and temporally close enough to be likely from a single neutrino interaction. 


\section{Formation of a track}

Tracks are reconstructed in a two step process: track finding and track fitting.

- Track finding: In this stage, the "seed tracks" or small segments of a candidate track, which consists of a number of hits in several proximal detector planes, are recognized by applying the Hough transform [104]. The track candidates are gradually constructed by adding the best track segments together.

- Track fitting: The track candidates from the finding stage are passed through a Kalman Filter [105] to find the best track candidates and estimate the preliminary parameters of track properties. This filter is basically the set of recursive equations that extrapolates and updates the dynamic state vector of muon at each point along the track. The effect of noise, multiple scattering and magnetic field are taken into account in order to decide if a given hit belongs to the track or not. If hits do not belong to the track, they will pass through as the input of the shower formation.

The state vector at each point along the track, after fitted by the Kalman Filter, consists of a transverse position in both $U$ and $V$ views, and transverse direction in two views $(d U / d z$ and $d V / d z)$. At the track vertex, the state vector additionally includes the estimated ratio of charge sign to momentum $(q / p)$ and its uncertainty $\left(\sigma_{q / p}\right)$ which is obtained by fitting 
the track trajectory in the magnetic field of the detector. If the track ends in the detector and not in the coil hole region, track momentum is calculated from the range with higher resolution. This method calculates the energy lost in the steel and the scintillator using the GEANT 3 simulation.

Figure 3.13 shows the muon track reconstructed efficiencies, defined as the ratio of the number of successfully reconstructed true muon tracks to the total number of true muon tracks. The efficiency at the Far Detector is expected to be higher than that at the Near Detector, due to the smaller event rate.

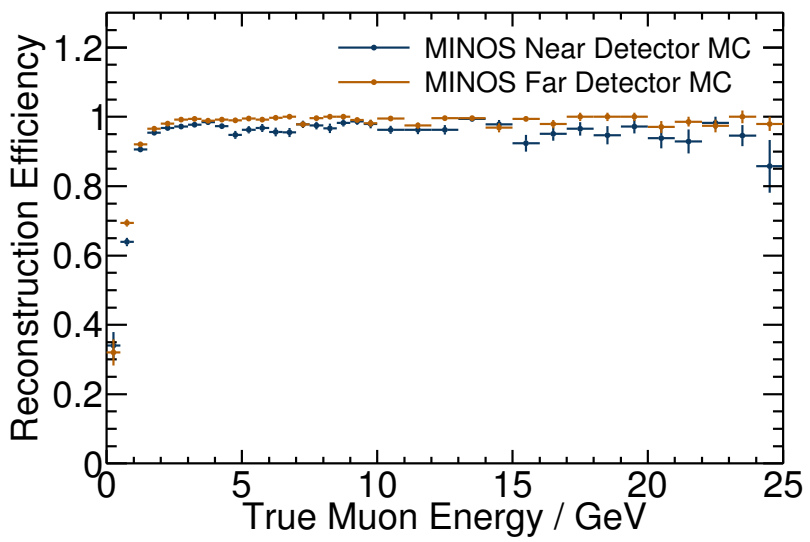

Figure 3.13: The muon track reconstructed efficiencies as the function of true muon track energy in two detectors of the MINOS experiments.

Although the track finding and fitting algorithms achieve a very high efficiency of muon track reconstruction, they also pass through a comparatively large number of non-muon tracks induced by the neutral-current interactions. Figure 3.14 shows composition of events with at least one reconstructed track. 

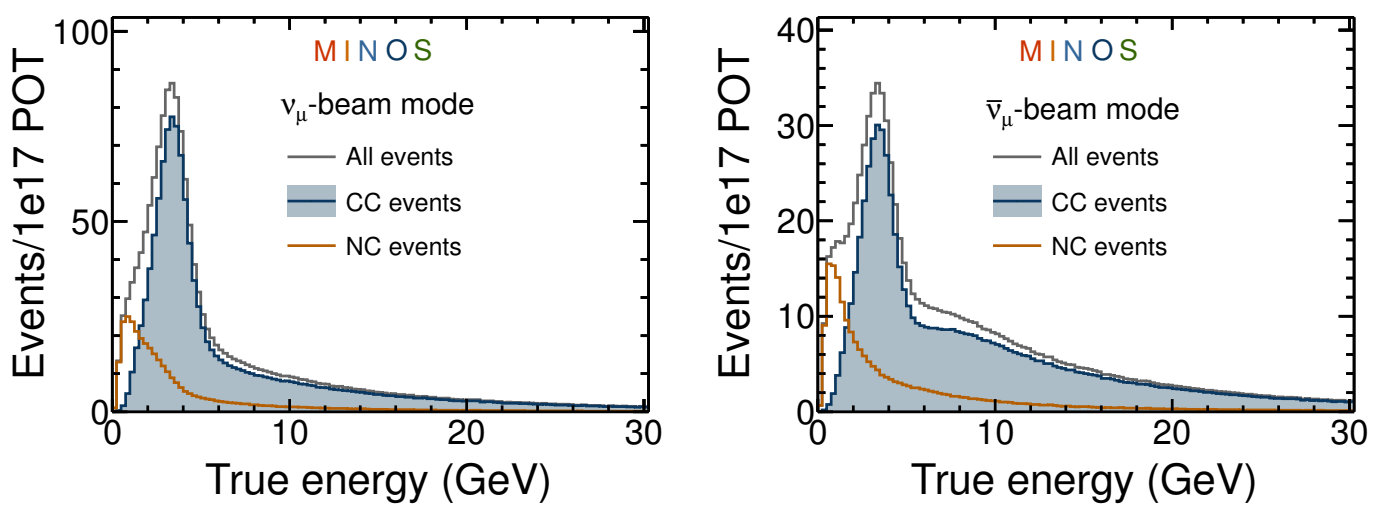

Figure 3.14: The composition of events having at least one reconstructed track in the $\nu_{\mu}$-beam mode (left) and $\bar{\nu}_{\mu}$-beam mode (right).

About $14-15 \%$ of events are from the neutral-current interactions. To reduce the contamination in the charged-current muon neutrino sample or in the neutral-current sample, multiple topological and dynamic features of the reconstructed track are exploited, as discussed in Chapter 4.

\section{Formation of a shower}

All remaining hits which do not belong to the track, are locally clustered together to form a number of candidate showers ${ }^{5}$. Each shower hit must have a summed pulsed height of at least two photoelectrons. This cut is necessary to remove the fiber noise and the PMT crosstalk. These clusters are then used to construct the shower of a single event by adopting an algorithm which takes advantage of non-clustered hits [106]. The transverse vertex position of the

\footnotetext{
${ }^{5}$ Track hits that have more energy than expected from the muon energy deposition, are also added to the shower.
} 
shower is assigned to the plane with the largest pulse height sum within 10 planes (5 planes in the same view) of the most upstream plane, along with its immediate upstream and downstream planes. The position of longitudinal shower vertex is the longitudinal coordinate of the scintillator in the most upstream plane. The shower energy is estimated generally by summing the energy deposited by all shower hits. Due to the coarse detector granularity, the shower energy resolution is poorer than the track energy resolution. The method of using a multivariate technique to estimate shower energy, discussed in Chapter 5, improves the shower energy resolution by combining additional information from the shower topology.

\section{Event building}

The individual neutrino events are constructed by pairing tracks and showers using their temporal and spatial proximity. In the most simple and frequent cases, an event consists of one track and one shower. If an event has multiple tracks and/or multiple showers, the primary track and the primary shower are determined based on a number of criteria ${ }^{6}$.

\footnotetext{
${ }^{6}$ Normally, the amplitude of pulse height deposition of track (shower) is considered and the highest one is assigned as the primary one.
} 


\subsection{The MINOS+ experiment}

The MINOS experiment finished its operations in April 2012. The two detectors continue to operate in the MINOS+ [107], in the medium-energy NuMI beam, which is optimized for the NOvA experiment. Due to the reconfiguration of the neutrino beam, the neutrino energy spectra is shifted to a higher energy, as shown in Figure 3.15. The energy spectra peak around 6$7 \mathrm{GeV}$ for both $\nu_{\mu}$-beam and $\bar{\nu}_{\mu}$-beam modes. Also, the beam composition has changed. Table 3.3 summarizes the beam compositions of the two beam modes in the MINOS and MINOS+ experiments. Compared to MINOS, the $\nu_{\mu}$-beam sample has purer $\nu_{\mu}$ events while $\bar{\nu}_{\mu}$-beam sample increases significantly the percentage of $\bar{\nu}_{\mu}$-CC events.

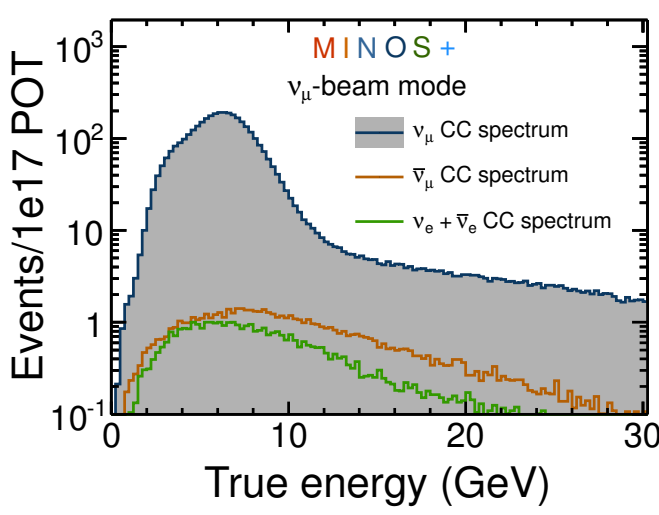

(a) $\nu_{\mu}$-beam mode

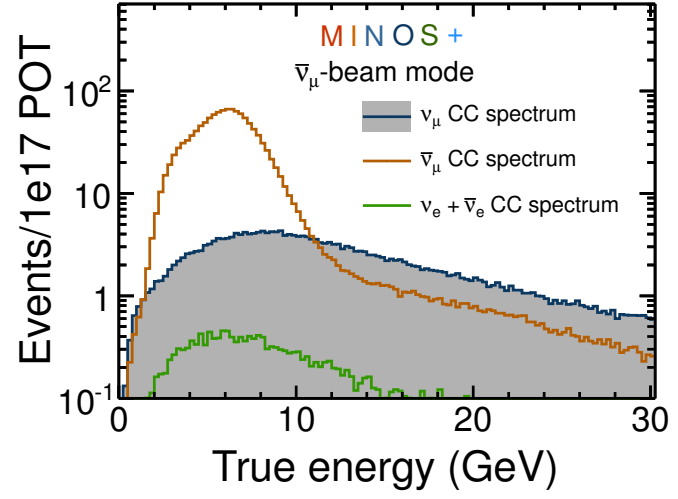

(b) $\bar{\nu}_{\mu}$-beam mode

Figure 3.15: The energy spectra of charged-current interactions for two beam modes in the MINOS+ experiment: $\nu_{\mu}$-beam (left) and $\bar{\nu}_{\mu}$-beam (right). The orange lines represent the $\nu_{\mu}$ CC events, the dark blue ones represent the $\bar{\nu}_{\mu^{-}}$ CC events, and the brighter blue ones present the $\nu_{e}$ and $\bar{\nu}_{e}$ events. 


\begin{tabular}{|c|c|c|c|c|}
\hline \multirow{2}{*}{ Components } & \multicolumn{2}{|c|}{ MINOS } & \multicolumn{2}{c|}{ MINOS+ } \\
\cline { 2 - 5 } & $\nu_{\mu}$-beam & $\bar{\nu}_{\mu}$-beam & $\nu_{\mu}$-beam & $\bar{\nu}_{\mu}$-beam \\
\hline$\nu_{\mu}$ CC $(\%)$ & 91.06 & 51.26 & 97.04 & 18.63 \\
\hline $\bar{\nu}_{\mu} \mathrm{CC}(\%)$ & 7.14 & 46.79 & 1.84 & 80.07 \\
\hline$\nu_{e}+\bar{\nu}_{e} \mathrm{CC}(\%)$ & 1.80 & 1.95 & 1.12 & 1.30 \\
\hline
\end{tabular}

Table 3.3: The comparison of charged-current event contents in the MINOS and MINOS+ experiments.

For the first three years of MINOS+ operations, we expect to collect more than 10,000 $\nu_{\mu}$-CC events and $3000 \mathrm{NC}$ events from an exposure of $18 \times 10^{20}$ POT. MINOS+ aims to search for non-standard interactions from the disappearance of $\nu_{\mu}$-CC events and sterile neutrinos from a NC rate study. Also, the large statistics of $\nu_{\mu}$-CC events at the high energy regions $(>5$ $\mathrm{GeV}$ ) provides MINOS+ a possibility for examining the effect of large-extra dimensions [59] with the neutrino oscillations. 


\section{Chapter 4}

\section{The event classification in MINOS}

This chapter describes two types of event selections used for the physics analyses in the MINOS experiment. Each selection is employed for a given

physics goal: charged-current muon neutrino $\left(\mathrm{CC}-\nu_{\mu}\right)$ event selection for the precision measurement of $\nu_{\mu}$ disappearance, and neutral-current (NC) event selection for the sterile neutrino search. The general idea of event selection is to compare the features of an input candidate to those expected of a particular signal and map this comparison to a quantitative parameter, which is assigned as the particle identification. The chapter will focus on the adoption of multi-variate techniques to enhance the performance of particle identification in MINOS.

\section{$4.1 \quad$ Event topologies}

In the MINOS experiment, neutrino interactions are broadly divided into three main types: $\nu_{\mu}-\mathrm{CC}$ interactions, $\nu_{e}-\mathrm{CC}$ interactions and $\mathrm{NC}$ interactions. 


\section{Charged-current $\nu_{\mu}$ interactions}

In this type of event, an incoming $\nu_{\mu}$ exchanges a $W$ boson with an iron nuclei in the detector to produce a muon and an accompanying hadronic shower. The muon loses its energy at a fairly constant rate and leaves a long curved track in the magnetized detector. Typically, a $1 \mathrm{GeV}$ muon track spans for 25 planes in the MINOS detectors. Figure 4.1 shows the Feynman diagram for $\nu_{\mu}$-CC interaction, along with event display for a characteristic event observed in the MINOS detector.
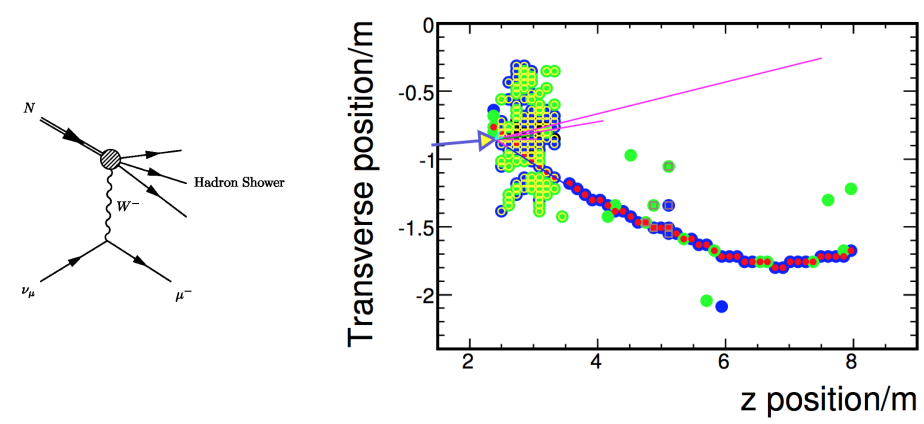

Detected energy depositions

Deposition $<2.0$ pe

- $2.0<$ Deposition $<20.0$ pe

- Deposition $>20.0$ pe

Reconstruction

- Reconstructed track hit

Reconstructed shower hit

Monte Carlo Truth

$\rightarrow$ Initial $v_{\mu}$

$=\pi^{ \pm} \quad-\pi^{+}$

Figure 4.1: The diagram of the $\nu_{\mu}$-CC interaction with nucleus (left) and the typical corresponding event reconstructed in the MINOS detector (right). The (green, blue, black) dots represent the reconstructed position of the scintillator strip with different amounts of deposited energy. The red and yellow circles are respectively the reconstructed track and shower hits. The solid line shows the true directions of particles.

The long muon track is the key feature to identify $\nu_{\mu}$-CC interactions. However, using only muon track length is not enough to efficiently classify this type of event. A comparatively large number of reconstructed short tracks are non-muon tracks, which are dominantly produced by the $\mathrm{NC}$ interactions. In 
order to efficiently reject the non-muon track background, a variety of muon track features are exploited and serve as inputs to a multi-variate algorithm, discussed in Section 4.4.

\section{Charge-current $\nu_{e}$ interactions}

This type of event has a dense electromagnetic shower at the interaction vertex and is normally surrounded by a sparser hadronic shower. Figure 4.2 shows the Feynman diagram for $\nu_{e}$ CC interaction, along with event display for a characteristic event observed in the MINOS detectors.
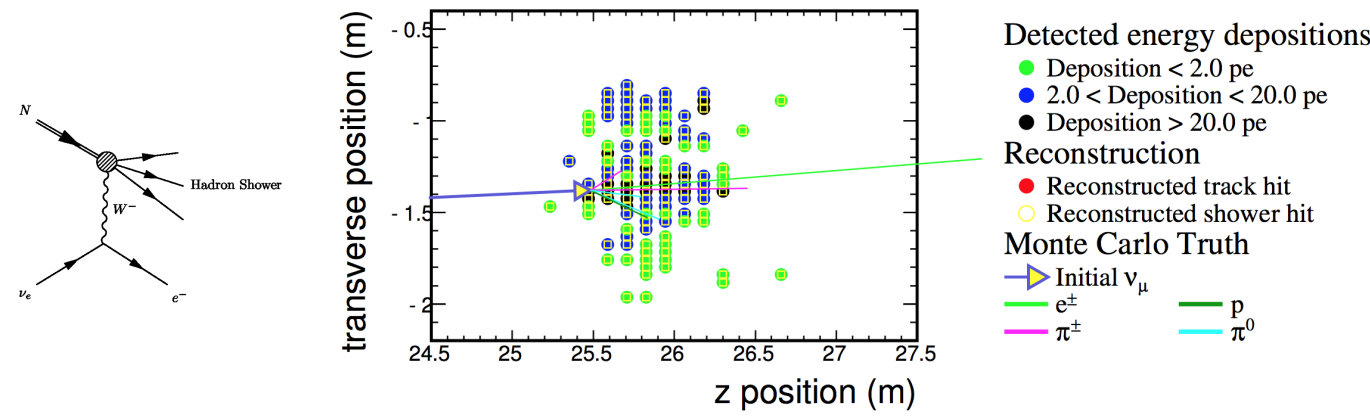

Figure 4.2: The diagram of the $\nu_{e}$-CC interaction with nucleus (left) and the typical corresponding event reconstructed in the MINOS detector (right). More information is in Figure 4.1.

The $\nu_{e}$ events come from two sources: "intrinsic" $\nu_{e}$ events from the NuMI beam $\left(1.8 \%\right.$ in the $\nu_{\mu}$-beam mode and $1.9 \%$ in the $\bar{\nu}_{\mu}$-beam mode) and "appeared" $\nu_{e}$ events from $\nu_{\mu}$ transitions. In the neutrino beam, the "intrinsic" $\nu_{e}$ events typically have higher energy than those of $\nu_{\mu}$ events. On the other hand, the "appeared" $\nu_{e}$ events from oscillations peak around $1.4 \mathrm{GeV}$, which 
is corresponding to the maximum of $\nu_{\mu}$ oscillation associated with the MINOS baseline. The main background for this analysis are the NC events. The MINOS detectors, with their coarse granularity, were not designed for selecting this type of event. A very sophisticated technique [108], is employed to classify this type of event.

\section{Neutral current interactions}

These interactions are meditated by the $\mathrm{Z}$ bosons and produce a number of sparse hadronic showers. Also, the resultant neutrinos leave the detector without leaving a track. Since the final state consists of neutrinos, energy of incoming neutrinos cannot be reconstructed. A typical NC event deposits $1 \mathrm{GeV}$ of energy in the detector with approximately 10 hits on the scintillator strips. Figure 4.3 shows the Feynman diagram for NC interaction, along with event display for a characteristic event.
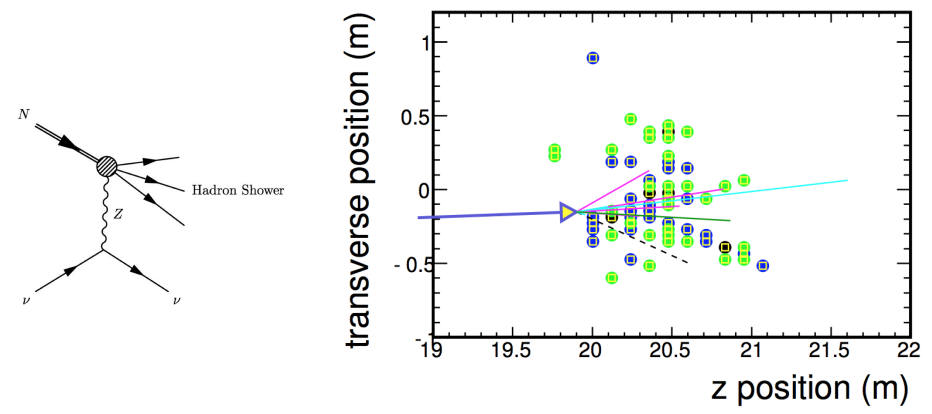

Detected energy depositions

Deposition $<2.0 \mathrm{pe}$

- $2.0<$ Deposition $<20.0 \mathrm{pe}$

- Deposition $>20.0$ pe

Reconstruction

- Reconstructed track hit

Reconstructed shower hit

Monte Carlo Truth

$\rightarrow$ Initial $v_{\mu} \quad \cdots$.. Outgoing $v$

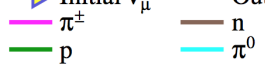

Figure 4.3: The diagram of the NC interaction with nucleus (left) and the typical corresponding event reconstructed in the MINOS detector (right). More information is in Figure 4.1. 
Since the cross-section of NC interactions are identical for the three active neutrino flavors, the $\mathrm{NC}$ event rates are not affected by the standard neutrino oscillations $^{1}$. Thus, study of the NC event rate provides a possibility to examine the existence of non-standard (sterile) neutrinos. We will discuss the NC selection in Section 4.5.

The magnetization of the MINOS detectors allows discrimination of $\mu^{+}$ tracks from $\mu^{-}$tracks via their curvature. Figure 4.4 shows event display of characteristic $\nu_{\mu}$-CC and $\bar{\nu}_{\mu}$-CC interactions observed in the MINOS detector.
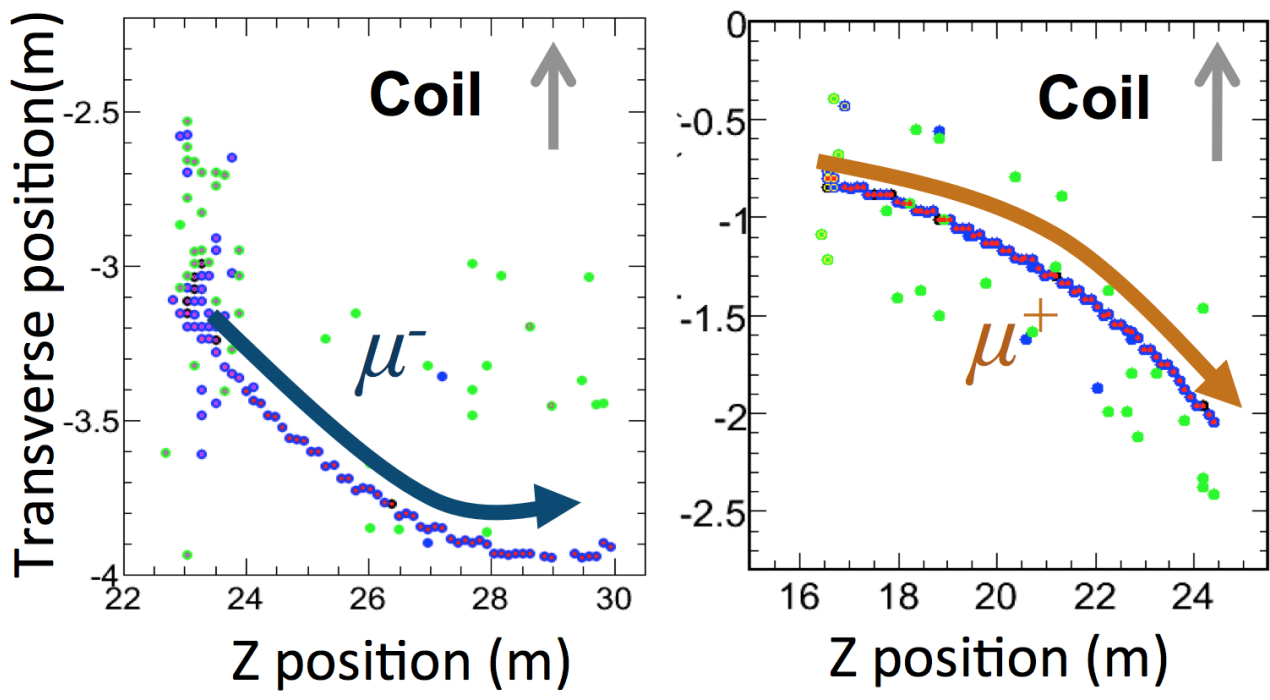

Figure 4.4: The discrimination of $\nu_{\mu}$-CC event (left) and $\bar{\nu}_{\mu}$-CC event (right) is recognized from the curvature of $\mu^{-}$and $\mu^{+}$in the detector. These events are simulated is the $\bar{\nu}_{\mu}$-beam mode, where the $\mu^{+}$is focused in to and $\mu^{-}$ defocused away from the coil hole.

\footnotetext{
${ }^{1}$ Regarding the oscillations between three active neutrinos
} 
The charge-sign measurement, which is briefly described in Section 4.2, is used to separate $\mu^{+}$and $\mu^{-}$tracks. Consequently, the $\bar{\nu}_{\mu^{-}} \mathrm{CC}$ sample and the $\nu_{\mu^{-}}$ CC sample can be isolated in an event-by-event basis, enabling independent measurements of the neutrino and antineutrino oscillation parameters.

\subsection{Muon charge-sign measurement}

The magnetization of the MINOS detectors enables one to distinguish the $\nu_{\mu}$-CC events from the $\bar{\nu}_{\mu}$-CC events via the curvatures of their induced $\mu^{-}$and $\mu^{+}$tracks respectively. The main uncertainty of charge-sign measurement is due to the Coulomb scattering of muons on their trajectory. The muon charge sign, $q$, and the muon momentum, $p$, are recorded in the state vector at the neutrino interaction verticies from the output of the Kalman Filter, as described in Section 3.7. These two variables are used to form the $q / p$ ratio, i.e., the reconstructed charge, which is assigned as the charge-sign measurement by default. Figure 4.5 shows the distributions of reconstructed charge of three types of events: $\nu_{\mu}$-CC, $\bar{\nu}_{\mu}$-CC and NC. The events with $q / p<0$ are considered as candidate $\nu_{\mu}$-like events while the events with $q / p>0$ are considered as $\bar{\nu}_{\mu}$-like events. Table 4.1 quotes the efficiency and purity of the chargesign measurement in two neutrino beam modes. The purity o charged-sign measurement of $\bar{\nu}_{\mu}$-CC events in the $\bar{\nu}_{\mu}$-beam mode is relatively low $(72.8 \%)$ because of the failure in determining the comparatively large proportion of high energy of $\nu_{\mu}$ events. Also, there are significant contributions from the neutral-current events. The separation of charge-current and neutral-current, 
discussed in the next section, will substantially remove the neutral-current events and consequently increase the signal purity in the final selected sample.

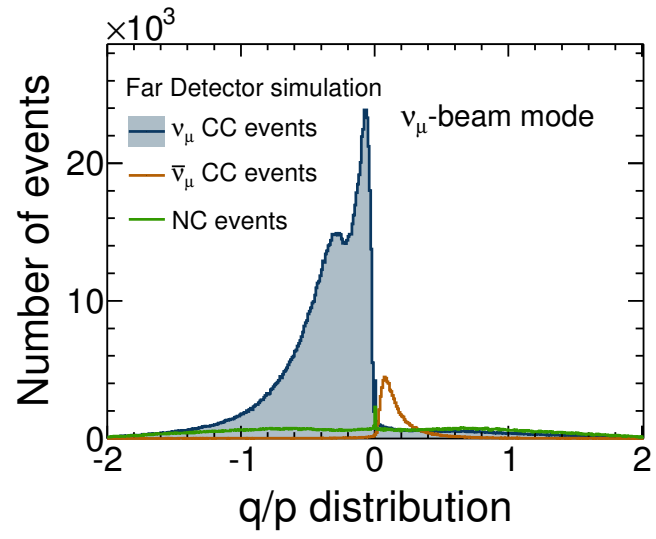

(a)

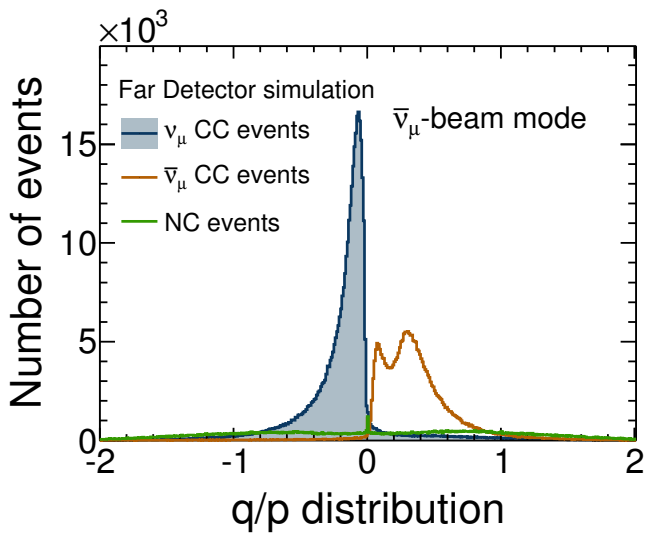

(b)

Figure 4.5: The $q / p$ distributions of $\nu_{\mu}$-CC, $\bar{\nu}_{\mu}$-CC and $\mathrm{NC}$ events in $\nu_{\mu}$-beam mode (left) and $\bar{\nu}_{\mu}$-beam mode (right) at the Far Detector.

\begin{tabular}{|l|c|c|}
\hline & $\nu_{\mu}$ in $\nu_{\mu}$-beam mode & $\bar{\nu}_{\mu}$ in $\bar{\nu}_{\mu}$-beam mode \\
\hline charge-sign efficiency & 0.926 & 0.975 \\
\hline charge-sign purity & 0.903 & 0.728 \\
\hline
\end{tabular}

Table 4.1: Performance of the charged-sign measurement for $\nu_{\mu}$ in the $\nu_{\mu}$-beam mode and $\bar{\nu}_{\mu}$-CC events in the $\bar{\nu}_{\mu}$-beam mode at the Far Detector. 


\subsection{Muon and non-muon track separation}

The primary signature of $\bar{\nu}_{\mu}$-CC interactions in the MINOS detectors are the reconstructed antimuon tracks. Since the antimuon track shares the identical topological features with the muon track except their curvature in the magnetized detector, an algorithm is developed to first identify the muon tracks without distinguishing between the $\bar{\nu}_{\mu}$-CC and $\nu_{\mu}$-CC events. Then, $\bar{\nu}_{\mu}\left(\nu_{\mu}\right)$-CC events are selected by requiring that those events have positive (negative) values of the reconstructed charge resulted by the charge-sign measurement, discussed in Section 4.2.

The separation of muon and non-muon tracks are developed by training their measured track features with a Monte Carlo simulation sample. In principle, the muon tracks deposit energy via the ionization process while the non-muon tracks are recognized by the interactions of hadronic showers. These two processes are separated by using the pulse height and the topology of track hits in the detectors. The highly distinguishable track features (input variables) are chosen and schematically mapped into one single separation parameter, called a particle identification discriminant (PID). Normally, two ways exist for mapping:

\section{Likelihood-based method}

A set of variables that characterize the event topology and kinematics is chosen and used to produce a set of probability density functions (PDF). This step is done with a large amount of events from the Monte Carlo samples. The 
probability for an observed (test) event to be CC-like and NC-like are then calculated by:

$$
P_{\mathrm{CC}}=P(\mathrm{CC}) \prod P\left(x_{i} \mid \mathrm{CC}\right), \quad P_{\mathrm{NC}}=P(\mathrm{CC}) \prod P\left(x_{i} \mid \mathrm{NC}\right)
$$

where $x_{i}$ is the $i^{\text {th }}$ input variable and $P(\mathrm{CC})$ and $P(\mathrm{NC})$ are the normalized factors. The PID is then defined by a majority vote:

$$
\mathrm{PID}=\frac{P_{\mathrm{CC}}}{P_{\mathrm{CC}}+P_{\mathrm{NC}}}
$$

This method was implemented for the measurement of the MINOS $\nu_{\mu}$ CC disappearance before 2008 .

\section{Multi-variate algorithm}

In this approach, multiple input variables from the Monte Carlo simulated events are used to build a multi-dimentional feature space. A pattern recognition algorithm is then applied to locally cluster those events and results in a decision rule for the event identity. Compared to the likelihood-based method, the multi-variate algorithm takes into account the correlations between input variables, which is important for event classification in the case of statistic limitation or non-paramatric correlation. Both the Artificial Neutral Network $(\mathrm{ANN})^{2}$ and k-Nearest Neighbors ( $\mathrm{kNN}$ ) algorithms have been used for the event classification in MINOS. The kNN algorithm [109] [110] is currently used for selecting $\nu_{\mu}$ charged-current and we will focus on this algorithm in the next sections.

\footnotetext{
${ }^{2}$ This algorithm is used for selecting $\nu_{e}$ and $\bar{\nu}_{e}$.
} 


\subsubsection{Topological features of muon tracks}

Generally, muons are approximately minimum ionizing particles which are described by the Bethe-Bloch equation [9]. The detailed discussion of the muon stopping power in the polystyrene scintillator can be found in [98]. Minimum ionization means the muon typically travels further than the nonmuon track in the detector. Also, muons lose energy at a fairly constant rate and their average energy deposition is consistent with the minimum ionizing particles $\left(\mathrm{MIPs}^{3}\right)$. In addition, a muon track is smoother and there is less scattering than the non-muon track. Figure 4.6 shows the difference of mean energy deposited per trip in muon tracks and non-muon tracks in two neutrino beams. The mean energies deposited by muon tracks are expectedly smaller and more stable than those of non-muon tracks.
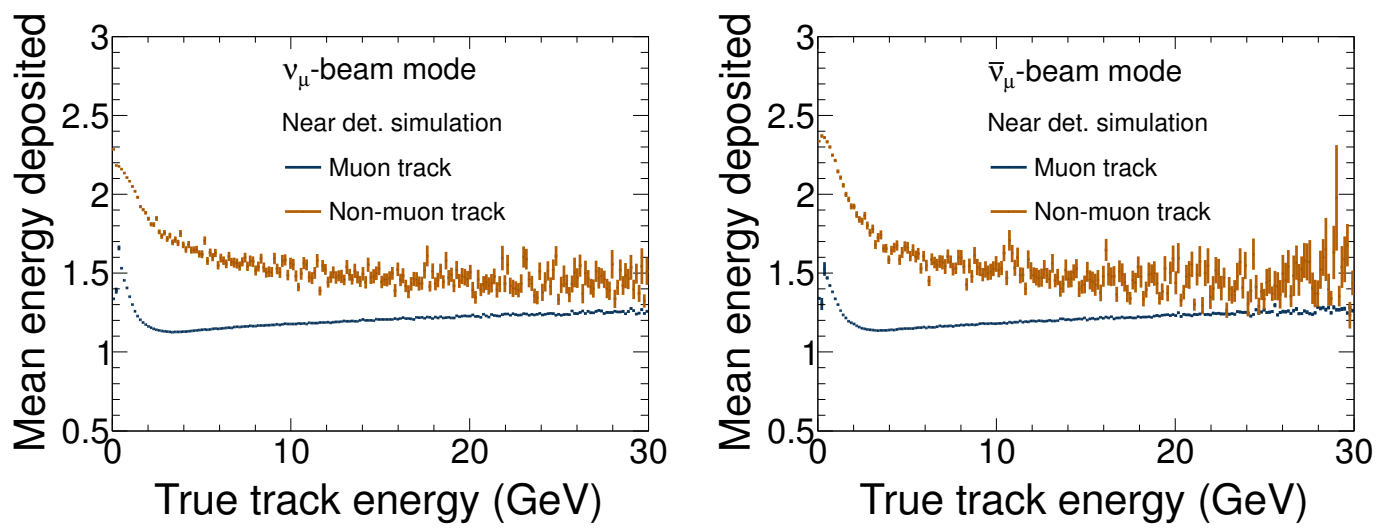

Figure 4.6: Mean energy deposited per strip in muon tracks (orang line) and non-muon tracks (blue line) in $\nu_{\mu}$-beam mode (left) and $\bar{\nu}_{\mu}$-beam mode (right) as the function of true track energy.

\footnotetext{
${ }^{3}$ In the MINOS detector, $1 \mathrm{MIP}$ is equivalent to $1.79 \mathrm{MeV}$ measured by stopping muon calibration [111]
} 
To develop an efficient muon track classification, multiple topological features of muon tracks are exploited. The following are brief descriptions of four topological variables which were developed specifically for identifying the muon tracks [109]:

- Number of track scintillator planes: Defined as the number of track planes containing scintillator strips that belong only to the track and not to any shower. This variable therefore measures the range of muon track within the detector and is strongly correlated to the track energy. Figure 4.7a shows the simulated distributions of number of track scintillator planes separately for muon and non-muon tracks at the Near Detector. On average, a muon track is longer than non-muon track.

- Mean pulse height of track hits: Defined as the average pulse height deposited on the track. Since muons are approximately minimum ionizing particles (MIPs), the distribution of mean pulse height deposited per strip in the track for muons, have a sharp peak near 1 unit of MIP. In contrast, the distribution for non-muon tracks has a much broader range, as illustrated in Figure 4.7b.

- Signal fluctuation: Defined as the fluctuation of energy deposited in the MINOS scintillator strip [109]. Compared to muons, hadronic showers have larger fluctuation in their deposited energy. By sorting the hits in an ascending order of pulser height and dividing it into two parts by 
a tunable parameter ${ }^{4}$, two average values of these two parts are calculated and the signal fluctuation is defined as the ratio between these two averages (low/high). Figure 4.7c shows the distributions of signal fluctuation separately for muon and non-muon tracks. On average, the signal fluctuation parameter distribution of muon tracks is higher and sharper than that of non-muon tracks.

- Transverse track profile: Defined as the ratio of the pulse height of the track hits to all of the event hits [109]. Typically, a muon deposits energy narrowly in a single scintillator strip while a hadronic shower scatters it across a few scintillator strips. Thus, if one considers a specific vicinity around the tracks, the transverse track profile of muon tracks evidently has a single peak while that of non-muon tracks has a broader range, as shown in Figure $4.7 \mathrm{~d}$.

These four topology variables are used to map into a single CC/NC separation parameter, called roID [109]. However, to maximize the sensitivity of muon track signature, all muon tracks with the number of scintillator planes smaller than 10 planes are removed by default. Thus, the roID ignores a substantial number of short-track events.

\footnotetext{
${ }^{4}$ This parameter is optimized by a sensitivity study
} 


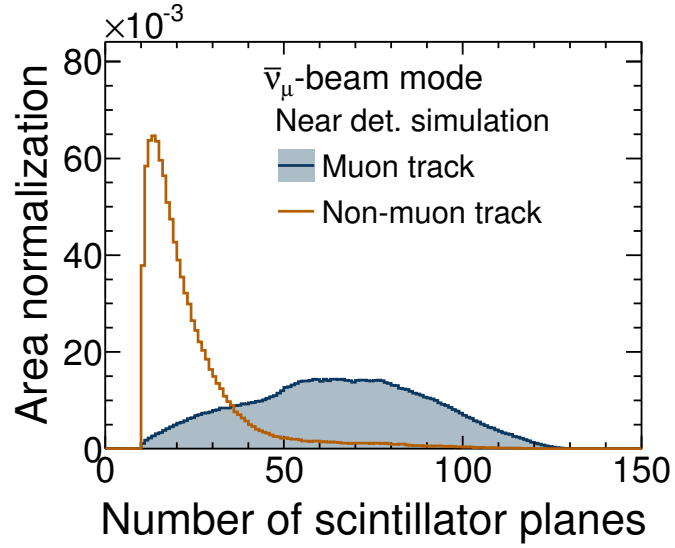

(a)

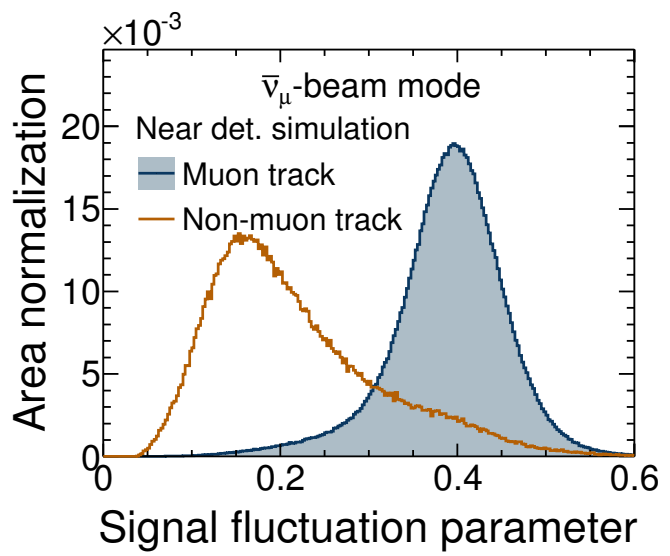

(c)

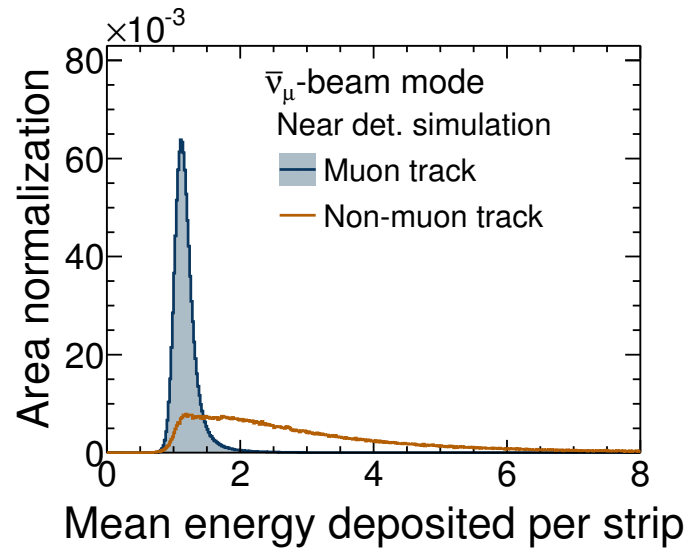

(b)

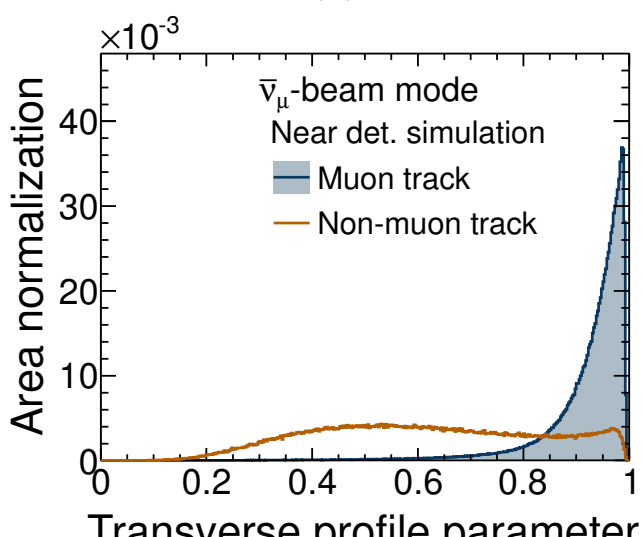

(d)

Figure 4.7: Distributions of four input topological variables simulated at Near Detector in the $\bar{\nu}_{\mu}$-beam mode. The muon tracks are shown in the blue histogram and the non-muon tracks are shown in the orange histogram. All distributions are area normalization. 
In an effort to recover the identity for these events, an alternative PID, called jmID [110], was developed and used as compensation for the roID ${ }^{5}$. The jmID is also constructed from four topological variables. The first one is the number of track scintillator planes, which was discussed earlier in this section but has been extended to include the short-track events. The three remaining variables: sums of track end pulse height and two degrees of scattering, are briefly introduced as follows:

- Sum of track end pulse height: While the pulse height of a proton or pion track increases dramatically at the end of track, the rising of the deposited energy of a muon is comparatively small. Thus the sum of pulse height at the track end can help to distinguish a muon track from a non-muon track. Figure 4.8 a shows the simulated distribution of track end pulse height separately for muon and non-muon tracks at the Near Detector.

- Two degrees of scattering: These two variables are defined by using the Pearson correlation coefficients of hit coordinates separately for $\mathrm{U} / \mathrm{Z}$ and $\mathrm{V} / \mathrm{Z}$ views. The Pearson coefficients, $\rho$, and degrees of scattering, $P_{\text {scattering, }}$, are defined as follows:

$$
\rho=\frac{\sum_{i} x_{i} z_{i}}{N \sigma_{x} \sigma_{z}}, \quad P_{\text {scattering }}=\frac{0.01}{1.01-\rho} .
$$

where $x$ and $z$ are the transverse and longitudinal positions of the hit and $\sigma_{x}$ and $\sigma_{z}$ are their corresponding distribution widths. The distributions

\footnotetext{
${ }^{5}$ The jmID has been used for selecting $\nu_{\mu}$-CC events but not $\bar{\nu}_{\mu}$-CC events
} 
of scattering degree are shown in Figure $4.8 \mathrm{~b}$ for $\mathrm{U}$ view and Figure $4.8 \mathrm{c}$ and for $\mathrm{V}$ view. On average, the degrees of scattering of non-muon tracks is smaller than its muon tracks. The degrees of scattering in the two views look identical due to the symmetrical $\mathrm{U} / \mathrm{V}$ readout.

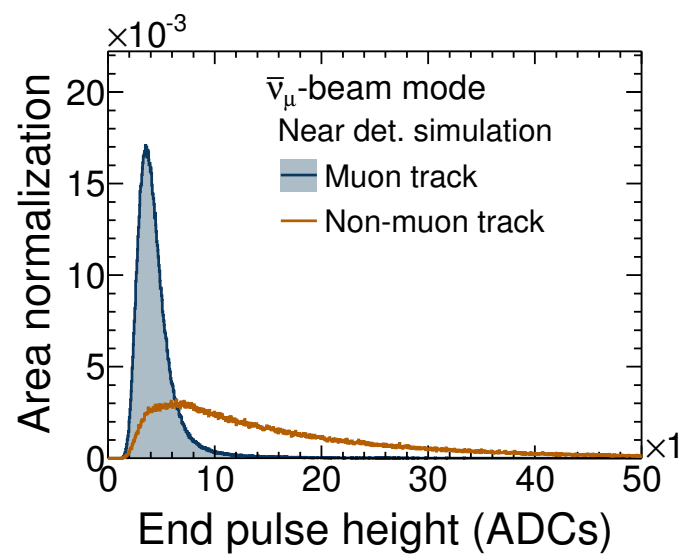

(a)

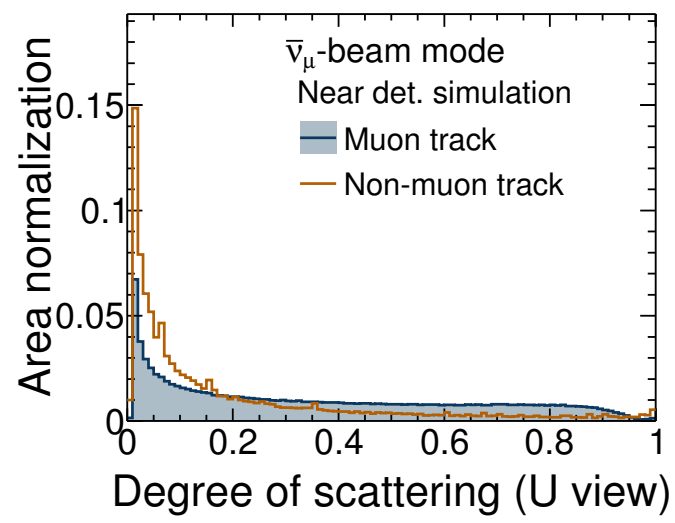

(b)

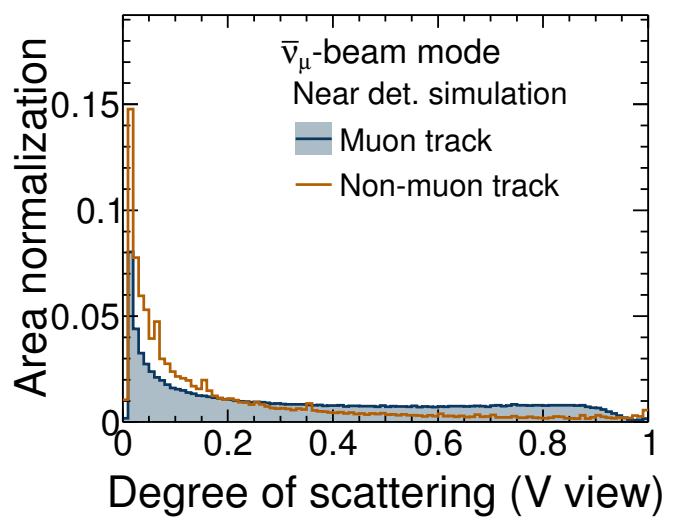

(c)

Figure 4.8: Three new variables introduced to build jmID: sum of end pulse heights (top) and degree of scattering in $\mathrm{U}$ view (bottom left) and in $\mathrm{V}$ view (bottom right) at the Near Detector. The muon tracks are shown in the blue histogram and the non-muon tracks are shown in the orange histogram. All distributions are area normalization. 


\subsubsection{The k-nearest neighbors algorithm for muon track identifica- tion}

The topological features described in the previous section show good separation between the muon tracks and the non-muon tracks. However, this separation is only observed with the Monte Carlo simulated events, in which their true track identity is known. This is not the case with observed events, from which we do not know the true information but want to discern identities. This is typical event classification with a supervised learning approach ${ }^{6}$.

First, we build a training set from a large number of Monte Carlo simulated events. Each event is recorded by multiple topological features (input variables) and labeled by its true track identity. In this particular case, events with true muon-track are considered as signals, and those of true non-muon track are backgrounds. Each event is essentially presented by one point in a multi-dimensional feature space in which each dimension is represented by an input variable. The separation of signal and background in each dimension results in a number of distinct, but normally overlapped, clusters for the signals and backgrounds in the multi-dimensional feature space. These clusters then provide a indication by which to estimate the decision boundary which is used as the prediction rule for each observed event. In many case, it is not straightforward how to find the decision boundary for a training set.

The k-nearest neighbor (kNN) algorithm [112] is found to be one of the most simple and robust multivariate algorithms for finding decision boundary

\footnotetext{
${ }^{6}$ this data learning is supervised since we have Monte Carlo simulated events as reference.
} 
of the event classification. For an observed event or arbitrary point in the multi-dimensional space, the kNN classifier uses a fixed number, $k$, of its nearest neighbors in the training set to make a decision. The nearest neighbors are determined by using the metric function in a multi-dimentional feature space. The simplest metric is the Euclidean distance defined by:

$$
R=\sqrt{\sum_{i=1}^{n_{\mathrm{var}}}\left(\left(x_{i}-y_{i}\right)^{2}\right)},
$$

where $n_{\mathrm{var}}$ is the number of input variables, $x_{i}$ is the coordinate of $i^{\text {th }}$ input variable of events in the training set, and $y_{i}$ are the coordinate of $i^{\text {th }}$ input variable of the observed events. Assuming that $k_{S}$ out of $k$ nearest neighbors are the signals, fraction $k_{S} / k$ is assigned as a discriminant parameter for that observed event. The value of discriminant parameter is between 0 (background-like) and 1 (signal-like). The decision boundary is then simply a hyper-surface of every point have the same value of discriminant parameter.

The kNN classifier gives the best performances when the boundaries that separate signal and background events have irregular shapes which cannot be easily approximated by parametric learning methods. Figure 4.9 shows the very well-adapted decision boundary of a $\mathrm{kNN}$ classifier for four types of separation data sets. 


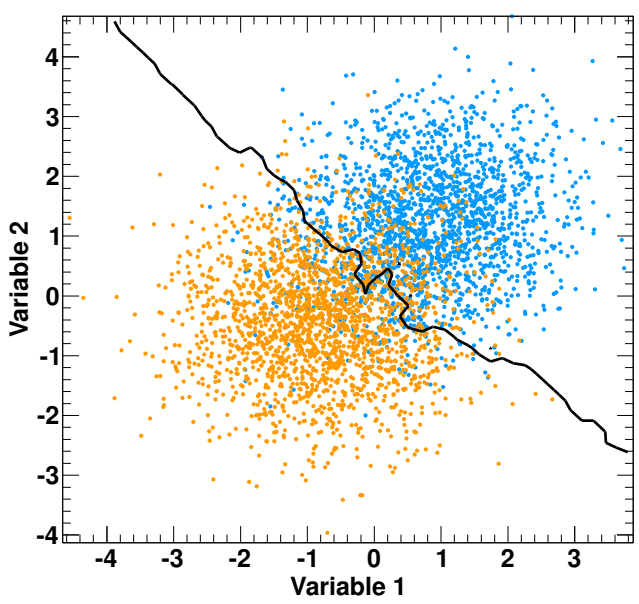

(a)

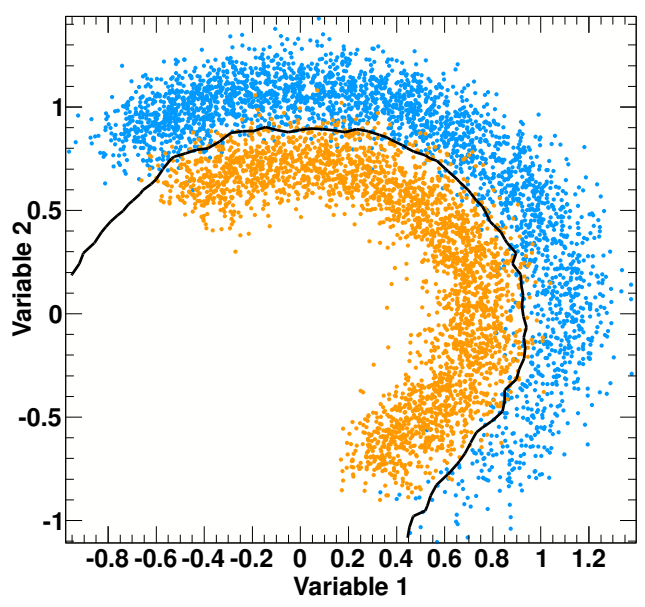

(c)

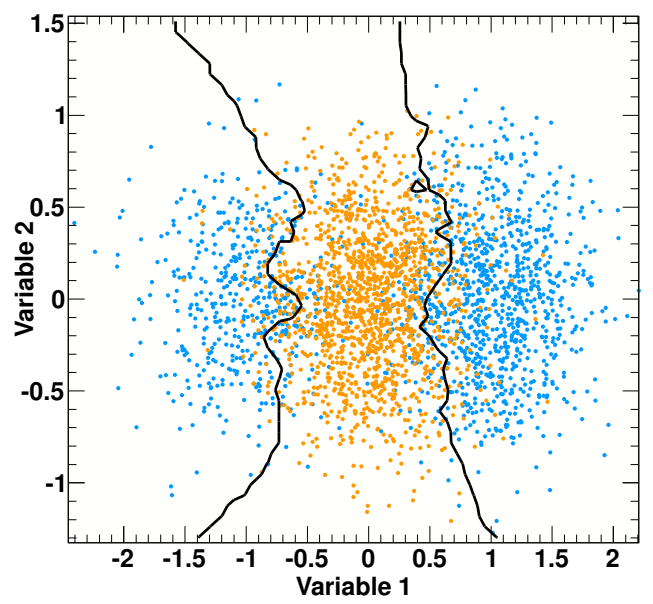

(b)

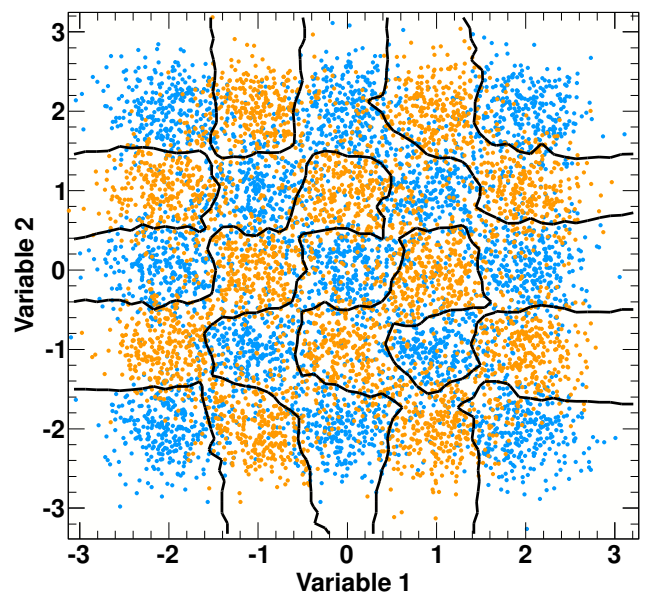

(d)

Figure 4.9: Decision boundary of kNN algorithm with different types of separation data sets of signal in blue and background in orange: (a) linear correlation, (b) three-bumps, (c) circle and (d) 2D-schachbrett. 
To evaluate the performance of even classification, we define signal efficiency and background rejection as follows:

$$
\begin{aligned}
& \text { Signal efficiency }=\frac{\text { Number of signals in selected sample }}{\text { Total number of signals }}, \\
& \text { Background rejection }=\frac{\text { Number of rejected background }}{\text { Total number of background }} .
\end{aligned}
$$

The performance of the $\mathrm{kNN}$ algorithm is compared to other supervising multivariate (MVA) algorithms by using the Toolkit for Multivariate Data Analysis (TMVA) [113]. Figure 4.10 shows a favor of the kNN algorithm when using four muon topological variables described in Section 4.3.1. With the same signal efficiency, the kNN classifier shows higher background rejection.

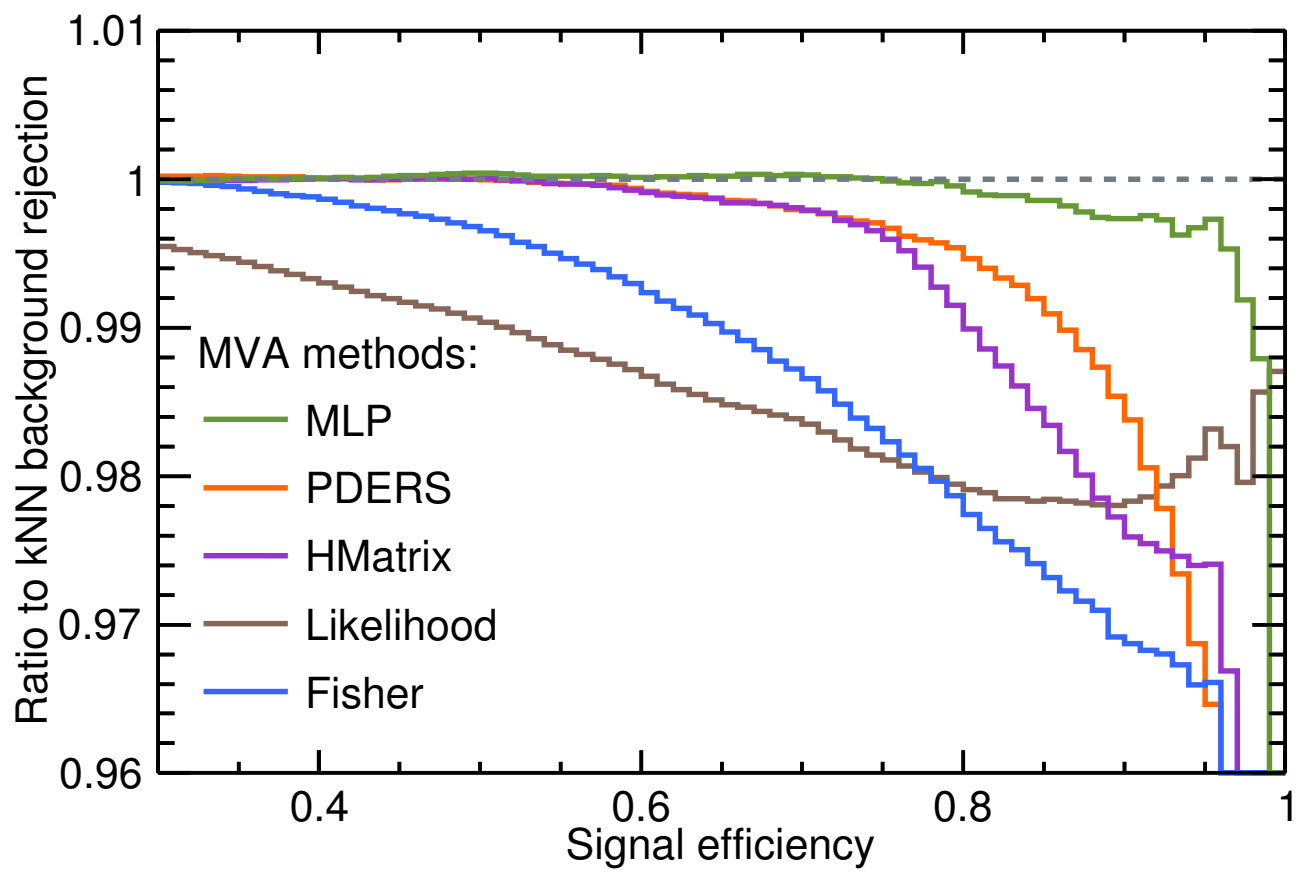

Figure 4.10: Compare kNN performance with other MVA methods. The y axis is the ratio of MVA background rejection to its of kNN algorithm. 


\subsubsection{Results of muon track identification}

The distributions of muon/non-muon (CC/NC) separation parameter, in comparison with the data and the Monte Carlo simulation at the Near Detector, for both two beam modes are shown in Figure 4.11. $k=80$, i.e for 80 assigned nearest neighbors for each observed event, is used for both cases. With an optimized cut on the $\mathrm{CC} / \mathrm{NC}$ separation parameter ${ }^{7}, \nu_{\mu}$-CC events are selected with high signal efficiency and purity. The numbers are quoted in Table 4.2 while the percentages of signal efficiency and background contaminations are shown in Figure 4.12. The efficiency of muon track identification in the $\bar{\nu}_{\mu}$-beam mode is higher than that in the $\nu_{\mu}$-beam mode. This is mainly due to the difference of average neutrino energy in the two beam modes.

\begin{tabular}{|l|c|c|}
\hline & $\nu_{\mu}$-beam mode & $\bar{\nu}_{\mu}$-beam mode \\
\hline Signal efficiency & 0.882 & 0.928 \\
\hline Signal purity & 0.983 & 0.978 \\
\hline
\end{tabular}

Table 4.2: Signal efficiency and purity of CC selection performance for selecting events with muon track in the $\nu_{\mu}$-beam mode and the $\bar{\nu}_{\mu^{-}}$beam mode.

\footnotetext{
${ }^{7}$ roID $>0.25 \|$ jmID $>0.5$ is used in the $\nu_{\mu}$-beam mode and roID $>0.3$ is used in the $\bar{\nu}_{\mu}$-beam mode.
} 

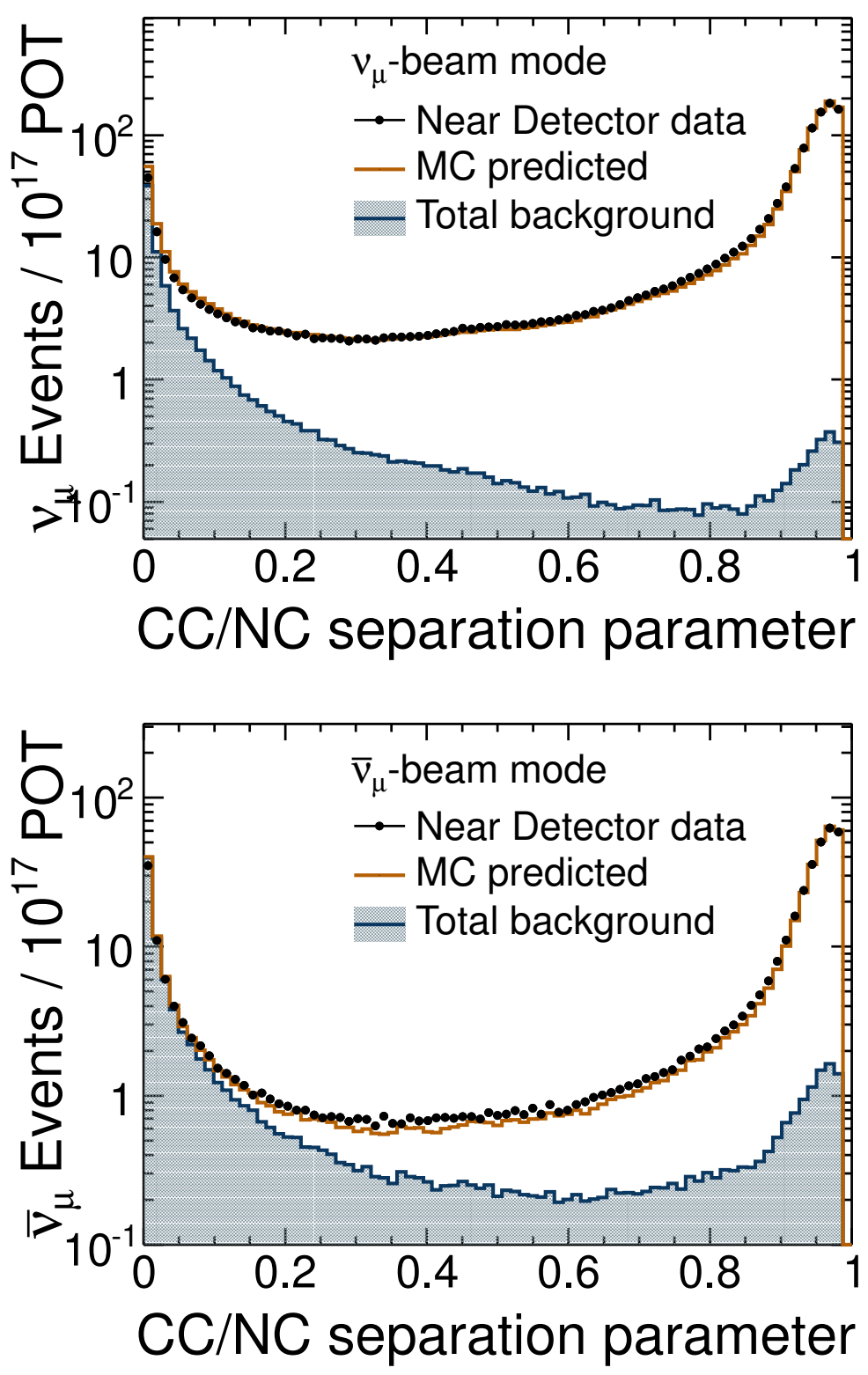

Figure 4.11: The CC/NC separation parameters are ploted for $\nu_{\mu}$-beam mode (top) and $\bar{\nu}_{\mu}$-beam mode (bottom) at the Near Detector. The data are shown as black dots. The Monte Carlo prediction are shown in the orange histogram and the background are shown in the blue histogram. 

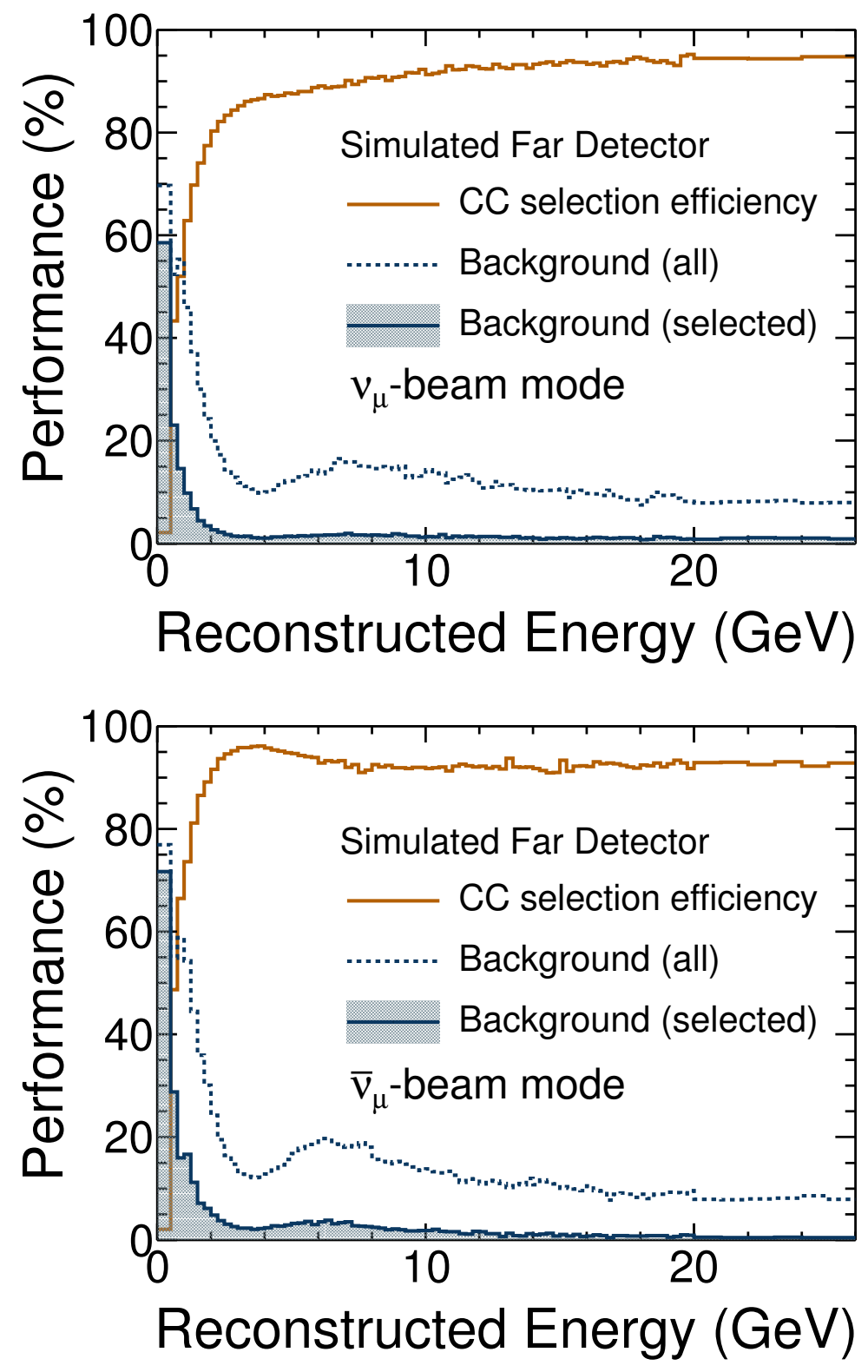

Figure 4.12: Performance of $\mathrm{CC} / \mathrm{NC}$ separation for selecting muon tracks in the $\nu_{\mu}$-beam mode (top) and the $\bar{\nu}_{\mu}$-beam mode (bottom). The signal efficiency is shown in the orange histogram. The background before selection (dashed blue histogram) and after selection (filled solid blue histogram) are included. 


\subsection{Charged-current $\bar{\nu}_{\mu}$ event selection}

The charged-current (CC) $\bar{\nu}_{\mu}$ events in the MINOS experiment come from three sources: $\bar{\nu}_{\mu}$-beam mode, $\nu_{\mu}$-beam mode, and atmospheric data. This section presents three corresponding event selections for $\bar{\nu}_{\mu}$-CC events.

\subsubsection{Selecting charged-current $\bar{\nu}_{\mu}$ events in the $\bar{\nu}_{\mu}$-beam}

After identifying the events with a muon track from the CC/NC separation parameter, $\bar{\nu}_{\mu}$-CC events are selected by requiring a positive value for the event charge-sign measurement, as described in Section 4.2. The performance of selecting $\bar{\nu}_{\mu}$-CC events in $\bar{\nu}_{\mu}$-beam mode are shown in Figure 4.13 while numbers are quoted in Table 4.3. Comparing with the $\nu_{\mu}$-beam mode, the selected events in the $\bar{\nu}_{\mu}$-beam mode contain a larger number of wrongsigned events. This is due to the original beam composition and the failure of charged-sign reconstruction. While the $\bar{\nu}_{\mu}$-beam mode includes $60 \%$ of $\nu_{\mu}$-CC events, the $\nu_{\mu}$-beam mode only contains $7 \%$ of $\bar{\nu}_{\mu}$-CC events. Also, the $\nu_{\mu}$-CC events with higher momentum follow a less-curved path, which increases the probability of failure during the charge-sign measurement for their track.

\begin{tabular}{|l|c|c|}
\hline & $\nu_{\mu}$ in $\nu_{\mu}$-beam mode & $\bar{\nu}_{\mu}$ in $\bar{\nu}_{\mu}$-beam mode \\
\hline Signal efficiency & 0.852 & 0.951 \\
\hline Signal purity & 0.989 & 0.921 \\
\hline
\end{tabular}

Table 4.3: Performance of selecting $\nu_{\mu}$-CC events in $\nu_{\mu}$-beam mode and $\bar{\nu}_{\mu}$-CC events in $\bar{\nu}_{\mu}$-beam mode. 

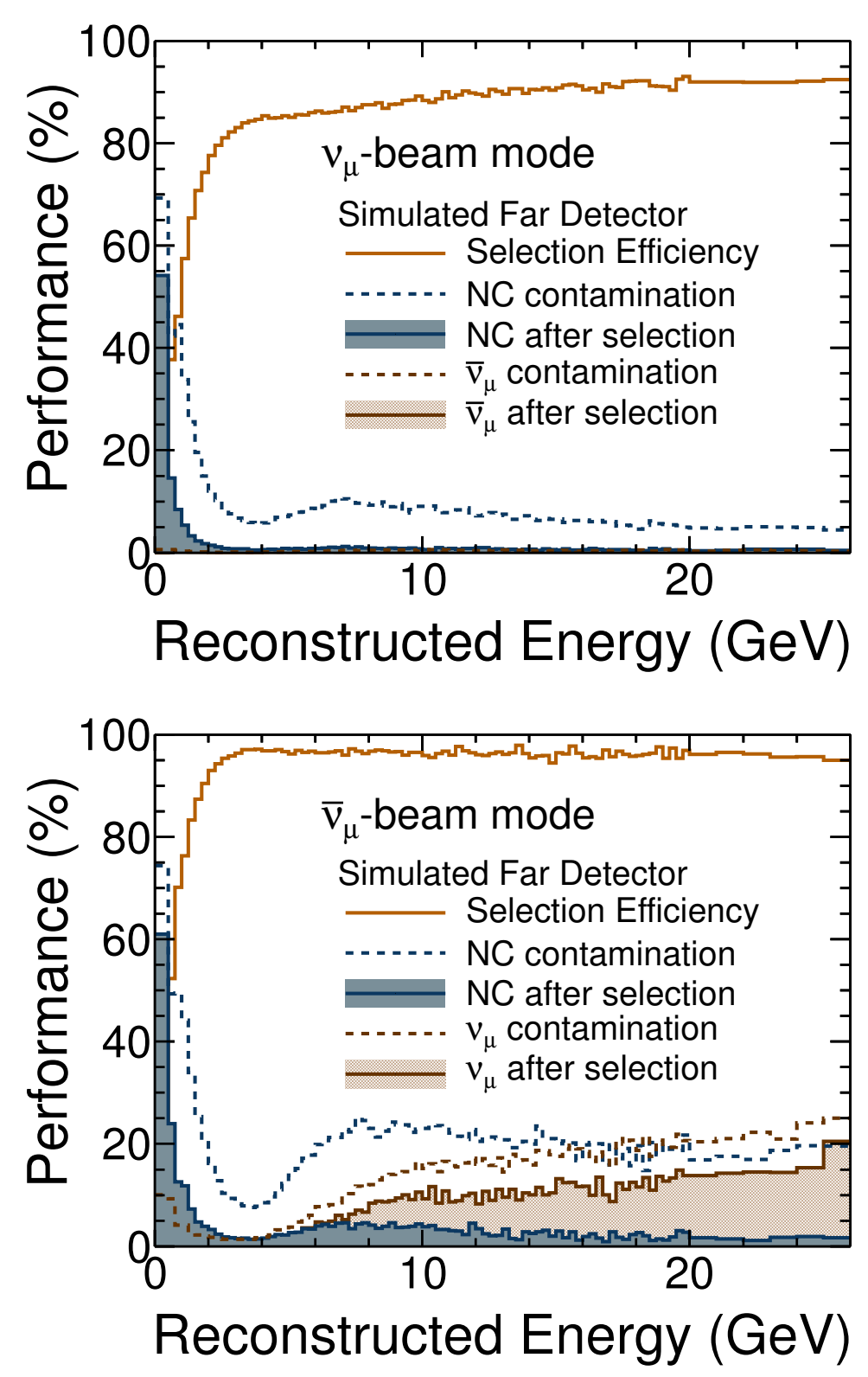

Figure 4.13: The signal efficiency and background contamination of selecting $\nu_{\mu}$-CC event in the $\nu_{\mu}$-beam mode and $\bar{\nu}_{\mu}$-CC events in the $\bar{\nu}_{\mu}$-beam mode. The signal efficiency is shown in the orange histogram. The NC background before selection (dashed blue histogram) and after selection (filled blue histogram) are included. The wrong-signed background before selection (dash brown histogram) and after selection (filled brown histogram) are presented. 


\subsubsection{Selecting charged-current $\bar{\nu}_{\mu}$ events in the $\nu_{\mu}$-beam}

The $\bar{\nu}_{\mu}$-CC events, which contribute about $7.1 \%$ of the $\nu_{\mu}$-beam mode, provide a unique test of the oscillation model for antineutrinos at the atmospheric scale [114]. These events statistically dominate at the $5-15 \mathrm{GeV}$ energy range, which is away from the observed dip of standard neutrino oscillation (about $1.5 \mathrm{GeV}$ ). To select $\bar{\nu}_{\mu}$-CC events, a simple approach is used for selecting events with a positive charge-signed track measurement from all candidate events with a muon-like track. However, this gives large contamination from $\mathrm{NC}$ events and wrong-signed events, as illustrated in Figure 7.23a. This is because the initial signal-to-background ratio of $\bar{\nu}_{\mu}$-CC events is much lower than that of $\nu_{\mu}$-CC events. A more sophisticated selection is necessary to achieve a highly pure sample of $\bar{\nu}_{\mu}$-CC events. The current selector we adopted is a sequence of cuts [115]. To get good $\mu^{+}$tracks, the events are selected with a good track fitter, where the ratio $\delta(q / p) /(q / p)>2.3$, and good track PID, roID $>0.65$. To reduce the neutral-current background, the $\mu^{+}$tracks requires to have long track length in both $\mathrm{U}, \mathrm{V}$ views, with both TrkEndV-TrkBeginV > 19 and TrkEndU-TrkBeginU $>19$. Also, the relative angle (described in the next section) is used to filter the defocused tracks, $\phi_{\text {relative }}>2.0$. Figure 4.14 shows the comparison of performance between two selectors: (a) cut on charge-signed measurement with a standard cut on PID, and (b) sequence of cuts described above. 


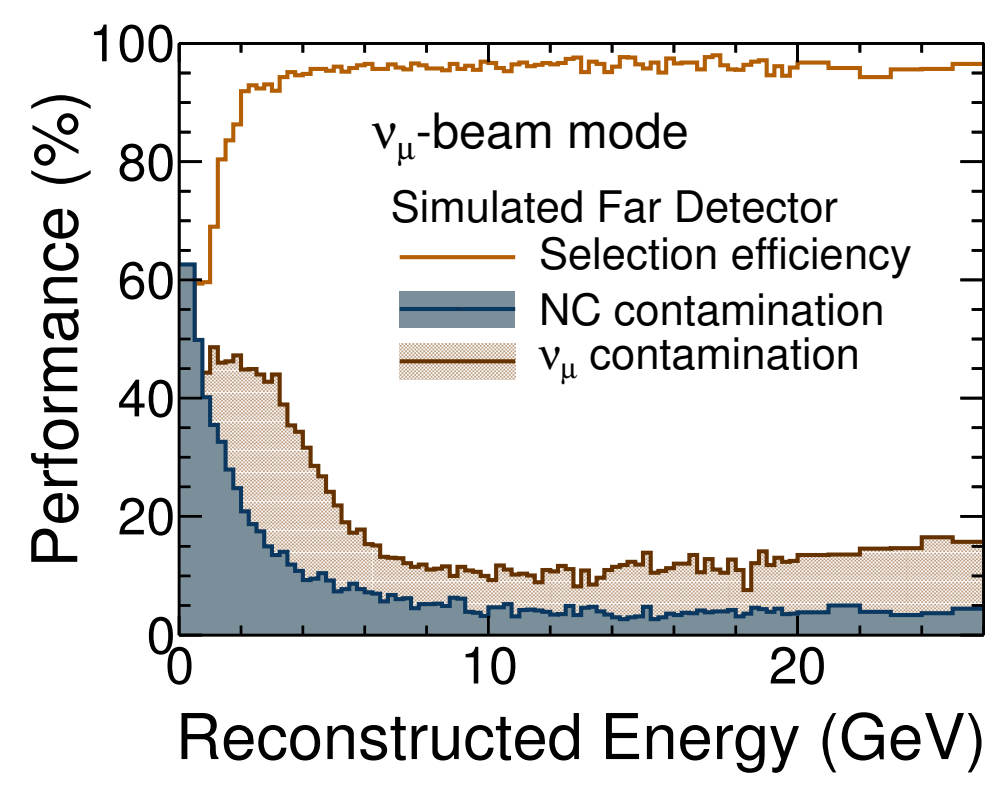

(a) Cut on charge-signed measurement

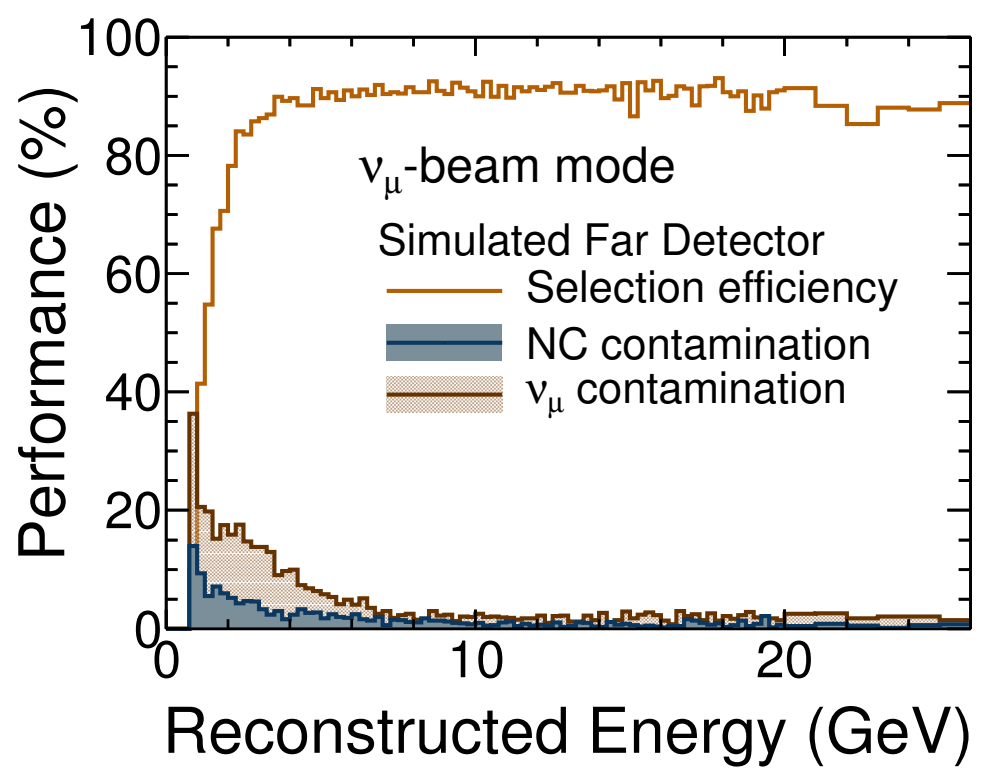

(b) Sequence of cuts

Figure 4.14: The performance of two selectors: cut on charge-signed track measurement (left), and sequence of cuts (right). The NC (blue histogram) and wrong-signed (brown histogram) contaminations are reduced significantly with later approach. 
Evidently, the sequence of cuts yield a much purer $\bar{\nu}_{\mu}$-CC sample but with a subtle decrease in signal efficiency, as shown in Table 4.4. The sequence of cuts is used for the measurement of $\bar{\nu}_{\mu}$-CC disappearance in this dissertation. This results in a small difference between the measurements in this dissertation in comparison to the MINOS recent published results (discussed in Section 7.6).

\begin{tabular}{|l|c|r|}
\hline & Simple approach & Sequence of cuts \\
\hline Signal efficiency & 0.951 & 0.878 \\
\hline Signal purity & 0.705 & 0.945 \\
\hline
\end{tabular}

Table 4.4: Performance of selecting $\bar{\nu}_{\mu}$-CC events in $\nu_{\mu}$-beam mode at the Far Detector with two approaches: cut on charge-signed track measurement (simple approach) and sequence of cuts. The former approach was used in the MINOS recent publication while the latter approach is used particularly for this dissertation.

\subsubsection{Improving the beam charged-current $\bar{\nu}_{\mu}$ event selection}

To estimate the amount remaining for improvement, a "magic" selection, in which there is no background contamination and the efficiency is very high, but not $100 \%$ due to the requirement of preselection of data (for example, the event has to have at least one good track...), is hypothetically defined. Figure 4.15 shows the comparison of the sensitivity contours with the "magic" selection alongside its current selection in the $\bar{\nu}_{\mu}$-beam mode. The room for event selection improvement is noteworthy. 


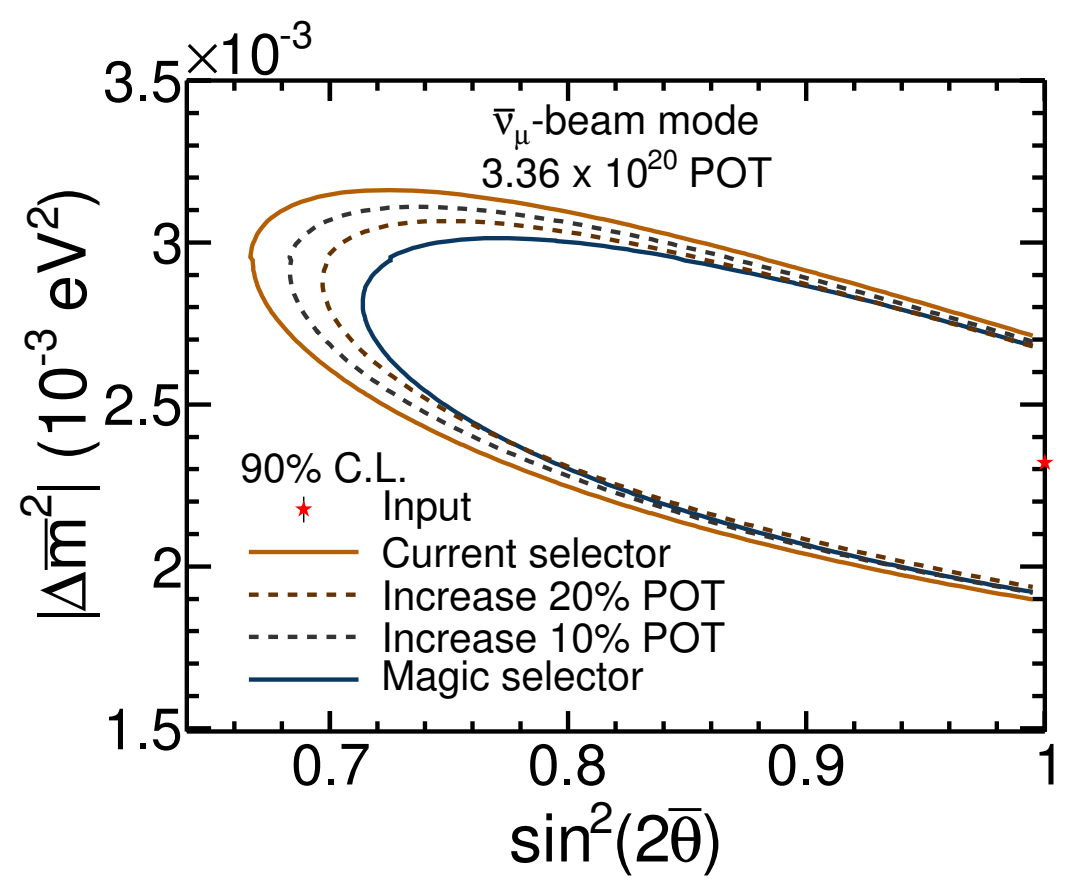

Figure 4.15: The 90\% C.L sensitivity contours with the magic selector (solid blue) and with the current selector (solid orange). The Monte Carlo sample is scaled to an exposure of $3.36 \times 10^{20} \mathrm{POT}$. The dashed blue and the orange contours are with official selection but with $10 \%$ and $20 \%$ respectively data increase.

The key for improving the $\bar{\nu}_{\mu}$-CC selection is to utilize both the topological and dynamic features of the muon. Also, instead of using a multi-variate algorithm for identifying muon tracks as a mediate step, we can directly classify the $\bar{\nu}_{\mu}$-CC events. In other words, in this event classification problem, the signal is the $\bar{\nu}_{\mu}$-CC events and background is the non- $\bar{\nu}_{\mu}$-CC events (including $\nu_{\mu}$-CC events). From the list of potential variables, six variables are used to build a new selection: 
1. CC/NC separation parameter: This variable, as shown in Figure 4.16a, takes the advantage of combining four muon topological variables and therefore has the power of distinguishing the $\mathrm{CC}$ events from the $\mathrm{NC}$ events.

2. Reconstructed charge: This variable is used as the final cut to specifically select $\nu_{\mu}$-CC or $\bar{\nu}_{\mu}$-CC events. However the mis-assigned events from this feature reduce efficiency and increase background contamination. Figure 4.16b shows the distributions of charge-signed measurement separately for three types of events: $\bar{\nu}_{\mu} \mathrm{CC}, \nu_{\mu}-\mathrm{CC}$ and NC. Employing this variable in correlation with other variables would reduce the misassignment of the events.

3. Relative angle: This variable is defined as the angle formed by two rays: the projection from the track vertex to the track end plane, and the projection with an assumption that there is no magnetic field and matter in the detector. Figure $4.16 \mathrm{c}$ shows the distributions of reconstructed relative angles for three types of events: $\bar{\nu}_{\mu} \mathrm{CC}, \nu_{\mu}-\mathrm{CC}$ and $\mathrm{NC}$.

4. Number of track plane: The NC events have peaks at lower energies while the $\mathrm{CC}$ events stretch out over the entire spectrum. Also this variable, as illustrated in Figure 4.16d, has small gain in distinguishing between $\bar{\nu}_{\mu}$-CC events from $\nu_{\mu}$-CC events due to the different energy ranges. 
5. Inelasticity (y) distribution: This variable is defined as a ratio between the reconstructed shower and the neutrino energy. Figure 4.16e shows the distribution of reconstructed inelasticity for three types of events: $\bar{\nu}_{\mu}$-CC, $\nu_{\mu}$-CC and NC. For the CC interactions, the reconstructed inelasticity is peaked towards one, particularly at lower energies. In contrast, the $\mathrm{NC}$ distributions are much flatter. This variable also has power of separation between $\bar{\nu}_{\mu}$-CC events and $\nu_{\mu}$-CC events since the inelasticity distribution of the latter, is flatter than that of the former.

6. Track radius: This variable is defined as the relative distance of track end and the track vertex on the $\mathrm{x}-\mathrm{y}$ plane:

$$
r_{\text {track }}=r_{\text {track end }}^{x y}-r_{\text {track vertex }}^{x y} \quad \text { where } \quad r_{i}^{x y}=\sqrt{x_{i}^{2}+y_{i}^{2}} .
$$

This variable also strongly correlates to the charge sign since the $\mu^{-}$and $\mu^{+}$tracks are expected to focus into and deflect away from the coil hole in the $\nu_{\mu}$-beam mode respectively and vice versa in the $\bar{\nu}_{\mu}$-beam mode. Figure 4.16f shows the distributions of the track radius for three types of events: $\bar{\nu}_{\mu}$-CC, $\nu_{\mu}$-CC and NC. While the track radius of $\bar{\nu}_{\mu}$-CC events are negative on average, the $\nu_{\mu}$-CC events has a peak at the positive value and the $\mathrm{NC}$ events has a peak at zero.

Figure 4.17 shows the comparison of the product of signal efficiency and signal purity between the current selector and new selector. The sensitivity gained by the new selector in comparison to the current selector, shown in Figure 4.18, is equivalent to a statistical increase of around $10 \%$ in the data. 


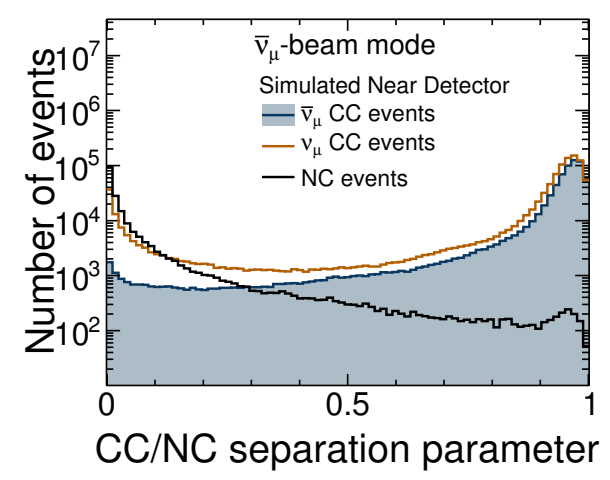

(a)

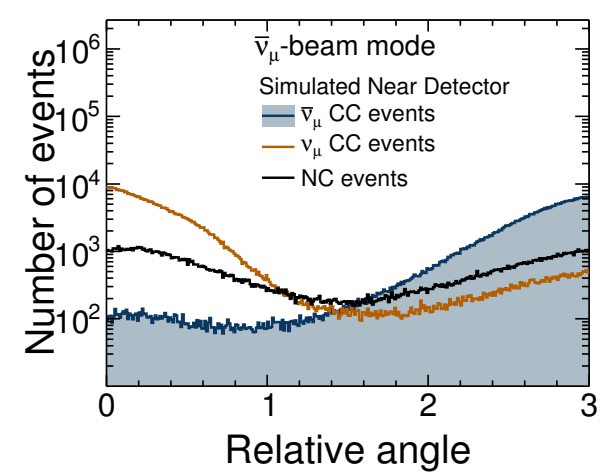

(c)

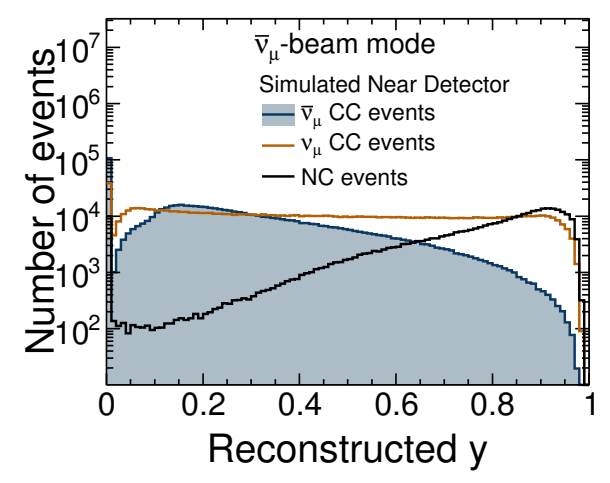

(e)

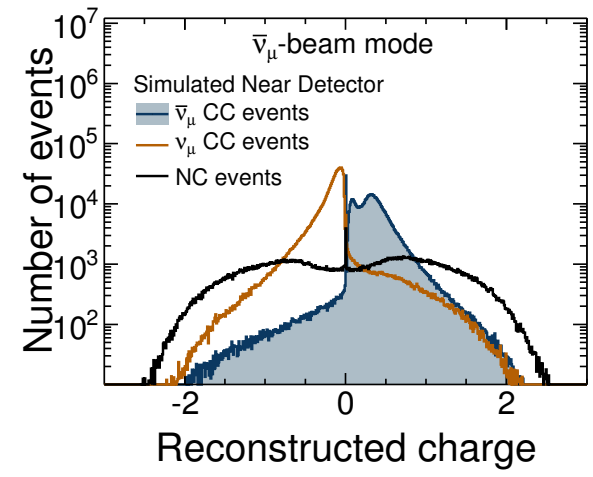

(b)

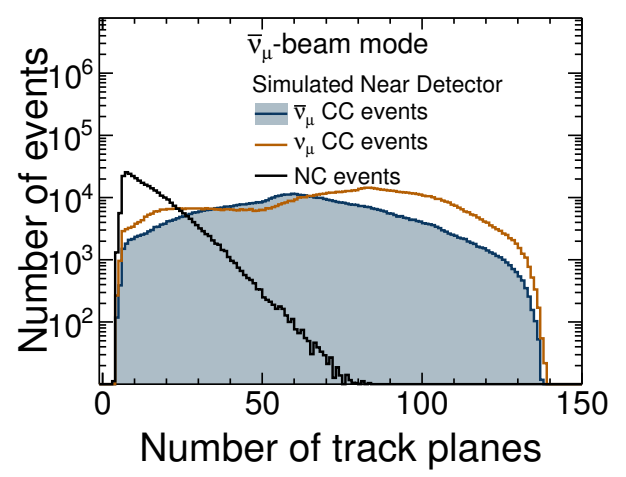

(d)

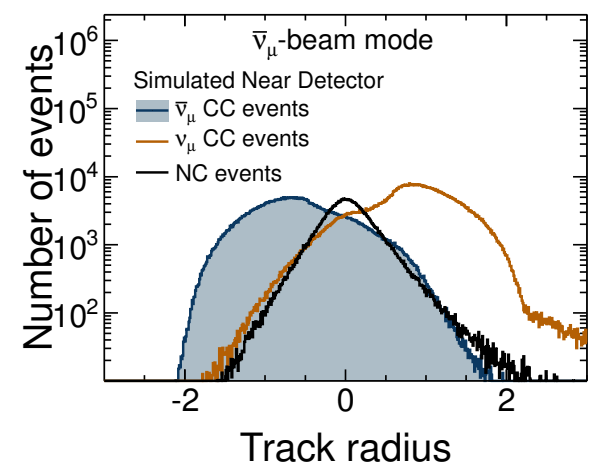

(f)

Figure 4.16: The distributions of six input variables: (a) CC/NC separation parameter, (b) reconstructed charge, (c) relative angle, (d) number of track planes, (e) reconstructed y, and (f) track radius, used for the new $\bar{\nu}_{\mu}$-CC event selection in the $\bar{\nu}_{\mu}$-beam mode. 


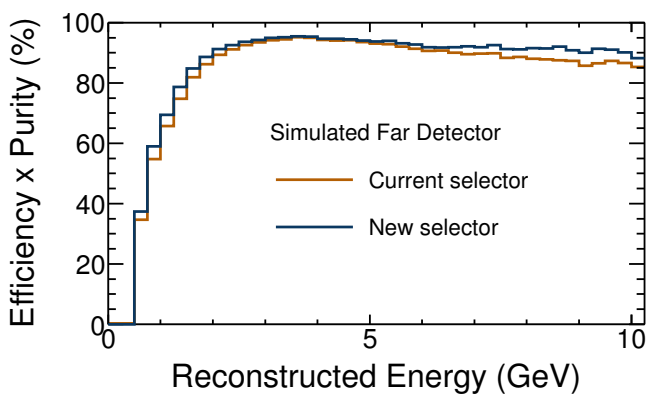

(a)

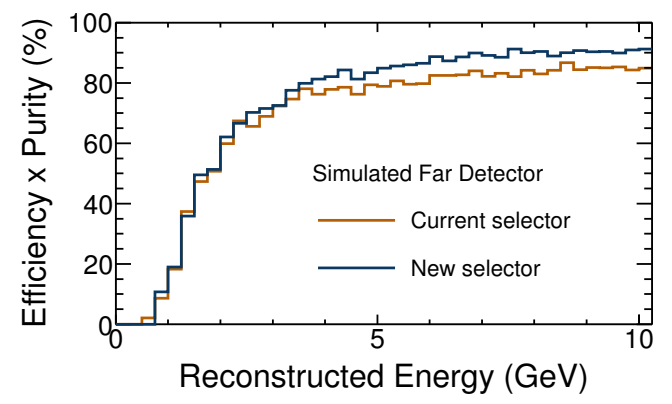

(b)

Figure 4.17: The products of the efficiency and sensitivity are compared between the official selector and the new selector for selecting $\bar{\nu}_{\mu}$-CC events in the $\bar{\nu}_{\mu}$-beam mode (left) and $\bar{\nu}_{\mu}$-CC events in the $\nu_{\mu}$-beam mode (right).

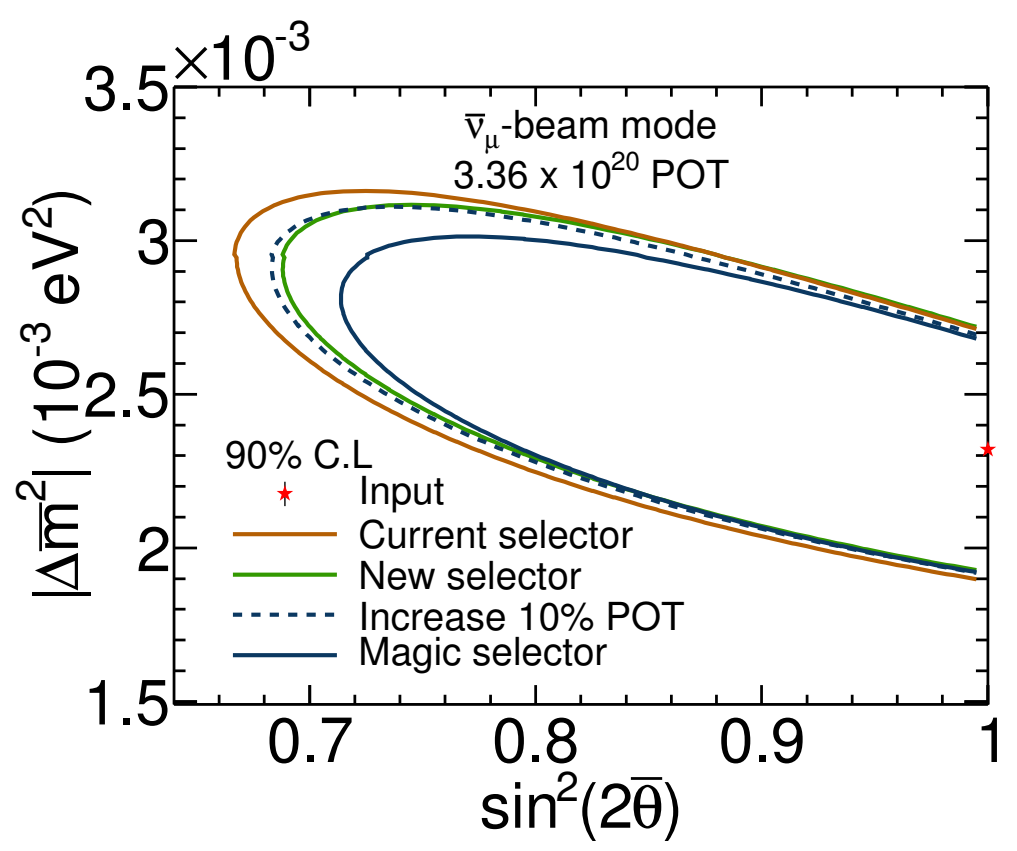

Figure 4.18: The $90 \%$ sensitivity contours of simulated $3.36 \times 10^{20}$ POT with new selector (blue), official selector (orange) and the magic selector (brown). The gain obtained by new selector is equivalent to a statistical increase of around $10 \%$ in the data (by matching with $10 \%$ POT increased contour in Figure 4.15). 


\subsubsection{Selecting atmospheric charged-current $\bar{\nu}_{\mu}$ events}

The main background for the atmospheric $\nu_{\mu}$ and $\bar{\nu}_{\mu}$-CC event selection is from cosmic-ray muons. A rate of 1 events/day atmospheric neutrino signals is much smaller than a rate of $10^{6}$ events/day cosmic-ray muons.

First, an atmospheric neutrino data sample is selected by removing the beam neutrino events within $100 \mu s$ time windows, which is extrapolated from the accelerator spill times. Also, full operation of both the Far Detector and veto shield is required to pass the collected events through. A tracklike sample, which contains events with track length of 8 or more planes, is sorted out. This sample is then used for selecting contained-vertex muons and neutrino-induced rock-muons, which are described as follows:

\section{Contained-vertex track selection}

To remove cosmic-ray muons in this sample, a set of containment and topology selection criteria is applied. Selected events need to be within a fiducial volume, which is required to be $0.2 \mathrm{~m}$ inside any edge of detector, $0.4 \mathrm{~m}$ from the coil hole center and 5 planes from the end of each super-module. Since the cosmic-ray muons are mainly incident from above with small angles to the detector planes, their average horizontal displacement along the z-axis, called trace $\Delta_{Z}$, is smaller than that of muons induced by the atmospheric muon neutrinos, as shown in Figure 4.19. Candidate atmospheric $\nu_{\mu}$ and $\bar{\nu}_{\mu}$-CC events need to satisfy $\Delta_{Z}>0.5 \mathrm{~m}$. 


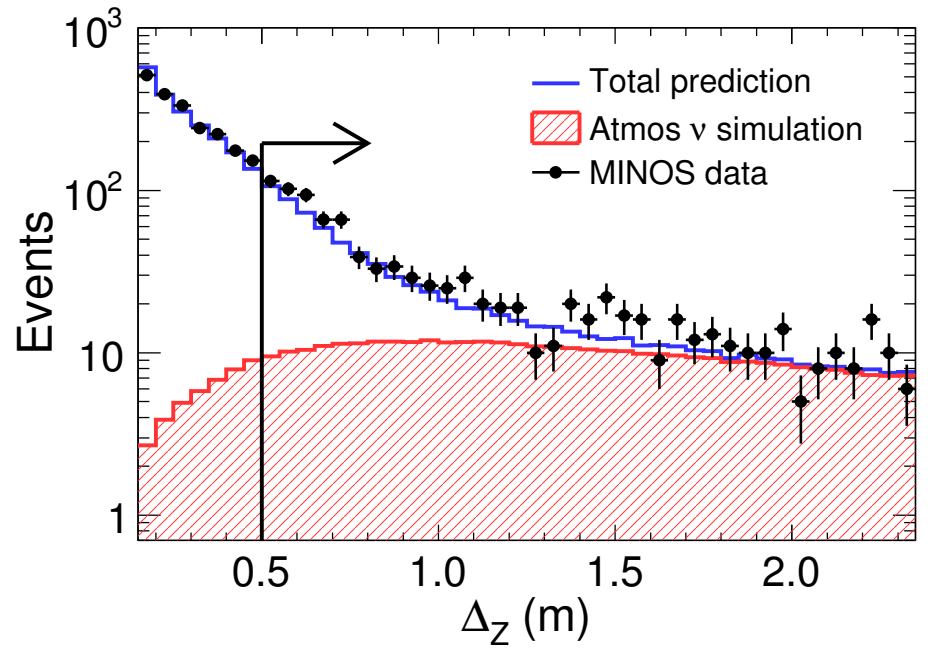

Figure 4.19: Distributions of the trace $\Delta_{Z}$ for contained vertex tracks. Data is shown in back, simulation of atmospheric neutrinos is shown in the red histogram and the total prediction is shown in blue.

In addition, a typical cosmic-ray muon deposits a large portion of energy at the upper end of its track due to a long traveling distance in the first detector plane. Therefore, a substantial number of cosmic-ray muon backgrounds can be removed by applying a cut on the pulse height at the upper end of track in correlation with the track directions. Figure 4.20 shows the distributions of the pulse height at the upper end of the track, plotted against the track direction projected on z-axis and y-axis. Events falling into the hatched region are rejected.

The contained-vertex track selection provides a $\nu_{\mu}$ and $\bar{\nu}_{\mu}$-CC sample of $92 \%$ in purity with $5 \%$ contamination from $\nu_{e}$ and $\bar{\nu}_{e}-\mathrm{CC}$ and neutral current events, and 3\% from cosmic-ray muon background. 

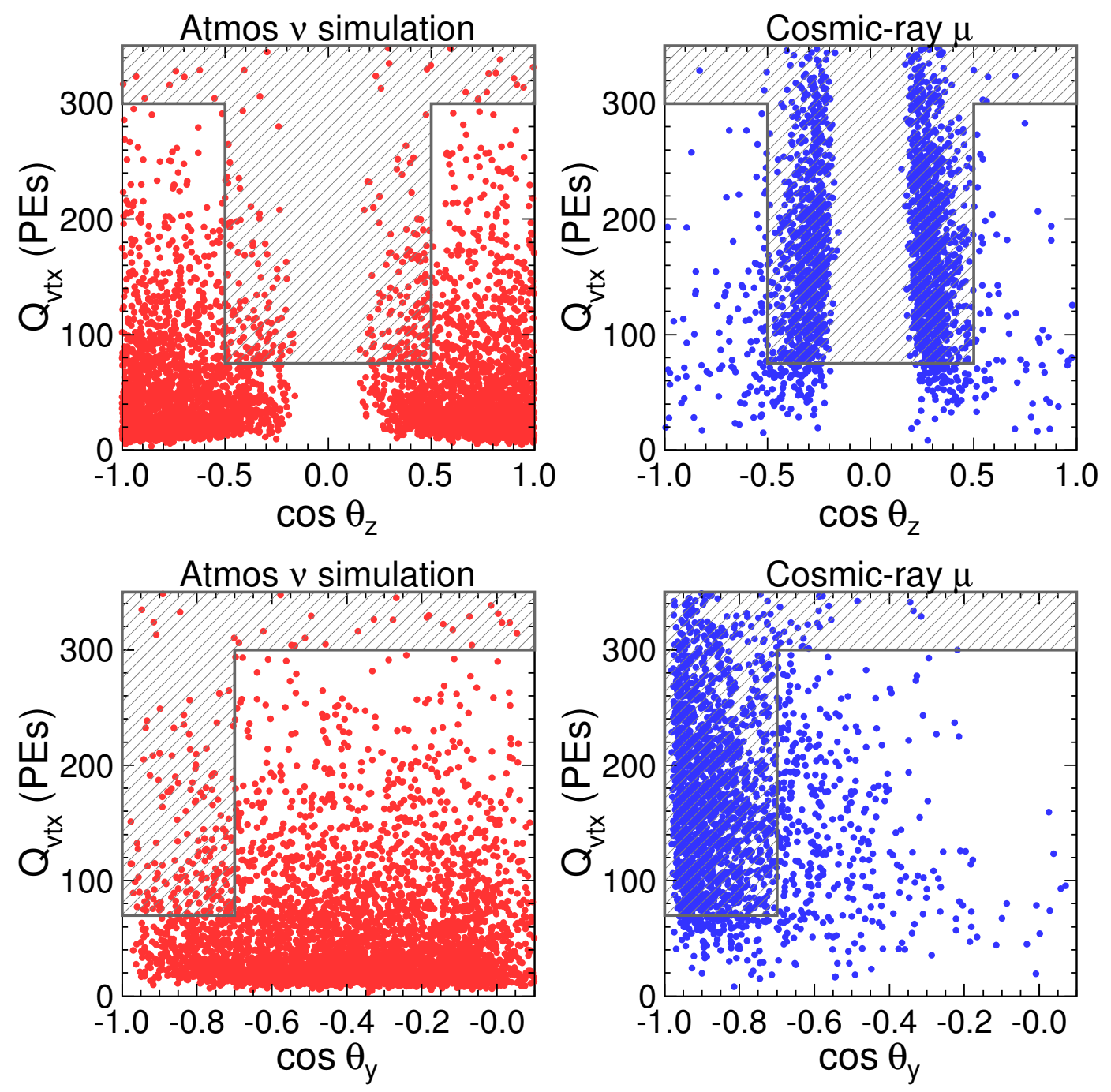

Figure 4.20: Distributions of the pulse height at the upper end of the track as a function of $\cos z$ (top) and $\cos y$ (bottom) for simulated atmospheric neutrinos (left) and cosmic-ray muons (right). Events falling into the hatched region are rejected. 


\section{Upward-going and horizontal track selection}

In this event sample, the cosmic-ray muon background is fairly small due to a shielding of approximate $14,000 \mathrm{~m}$ water-equivalent overburden. Thus, events with upward-going and horizontal tracks are primarily signatures of the atmospheric $\nu_{\mu}$ and $\bar{\nu}_{\mu}$-CC interactions. Since the track vertex can either be inside or outside of the fiducial volume, this sample includes both containedvertex muons and neutrino-induced rock muons. A two variable cut-based approach is used to select this type of event: (i) reciprocal muon velocity $(1 / \beta)$ and (ii) reconstructed zenith angle. The former variable is obtained by the gradient of linear fitting to the measured times as a function of distance along each track. Also, cuts on track topology and timing are required to ensure that the track direction is well-reconstructed. Figure $4.21 \mathrm{a}$ shows a good separation of the $1 / \beta$ distributions between the upward-going atmospheric muon neutrinos (peak at -1) and cosmic-ray muons (peak at +1 ). Distribution of the reconstructed zenith angles of selected events is shown in Figure 4.21b. The number of events decrease dramatically when $\cos \theta_{z}$ decreases from 1 to 0.1 , but become fairly flat when $\cos \theta_{z}<0.1$. This feature agrees with an

increase of the rock overburden when the zenith angles decrease. With a requirement of negative $1 / \beta$ and $\cos \theta_{z}<0.5$, the portion of background in this sample found to be insignificant in the subsequent analysis. 

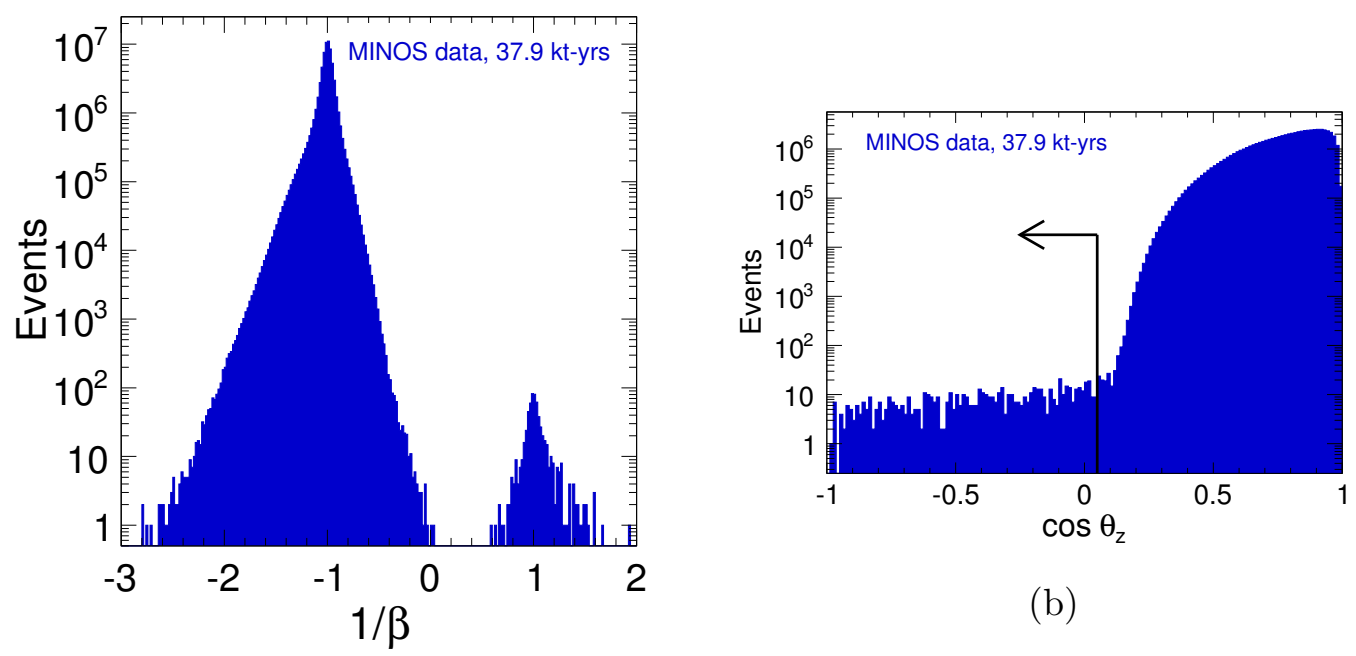

(b)

(a)

Figure 4.21: Distribution of reciprocal muon velocity, $1 / \beta$ (left) and distribution of zenith angle (right) for the selected events.

To distinguish $\bar{\nu}_{\mu}$-CC events from $\nu_{\mu}$-CC events, a significant track curvature is required for each event candidate. Two criteria are used to fulfill this requirement. The first one is the relative size of the track fit uncertainty $|q / p| / \sigma_{q / p}>2.5$. The second is the relative goodness of linear fit to the reconstructed track curvature $\chi_{\text {line }}^{2} /$ d.o.f $>4$. The efficiencies of charge-sign selection are $87 \%$ and $59 \%$ in the contained-vertex muon sample and neutrinoinduced rock-muon sample, respectively. The comparatively low efficiency in the latter sample reflects the fact that neutrino-induced rock-muons have a higher average momentum, resulting in higher probability of failure in chargesign measurement. 


\subsection{Neutral-current event selection}

The main background for the neutral-current (NC) selection is the $\nu_{\mu^{-}}$ CC events which have short or no reconstructed tracks with diffused hadronic activities. The subdominant background comes from both $\nu_{e}-\mathrm{CC}$ and $\nu_{\tau^{-}} \mathrm{CC}$ events.

\subsubsection{Preselection}

The existence of neutrinos in the final state makes it impossible to reconstruct the total energy of the $\mathrm{NC}$ events. The analysis essentially depends on how well we reconstruct the hadronic showers. The poorly reconstructed events, which are neglected for this selected sample, are defined in the Monte Carlo simulation as $E_{\text {reconstructed }}^{\text {shower }} / E_{\text {true }}^{\text {show }}<0.3$. Additionally, two more variables are used for preselecting the NC events:

- Maximum consecutive plane $\geq 3$ : We expect that the energy of hadronic showers is deposited in contiguous planes.

- Fraction of slice pulse height ${ }^{8}>0.5$ : This variable is used to measure the concentration of energy deposition.

\footnotetext{
${ }^{8}$ Slides are defined as the time and space windows in which the energy deposition activities are concentrated
} 


\subsubsection{Main selection}

The main part of the NC selection is based on a two variable cut-based approach. These two variables are briefly described as follows:

- Event length: Any events with an event length of more than 47 planes are removed. This significantly reduces the number of $\nu_{\mu^{-}} \mathrm{CC}$ events which are typically characterized by long muon tracks. Figure $4.22 \mathrm{a}$ shows the distribution of event length of NC events in the Near Detector compared to the Monte Carlo prediction.

- Track extension: This variable is defined by subtracting the shower extension from the track extension. For $\nu_{\mu}$-CC events, value of the track extension should be large because of the long muon track, while the NC events with a short track and horizontally developed shower, are characterized by a much smaller extension. Any events with a track extension larger than 6 are removed from sample, as illustrated in Figure 4.22b. The events with no track pass through this cut by default.

The position of the cuts for these two variables is optimized by maximizing $\epsilon \times p /(2-p)$ configuration of merit where $\epsilon$ is the signal efficiency and $p$ is the signal purity. The selected NC event sample after this two variable basedcut selection has an efficiency of $89 \%(80 \%)$ and purity of $61 \%(72 \%)$ for the $\nu_{\mu}$-beam $\left(\bar{\nu}_{\mu}\right.$-beam $)$ modes at the Far Detector $[60]$. 


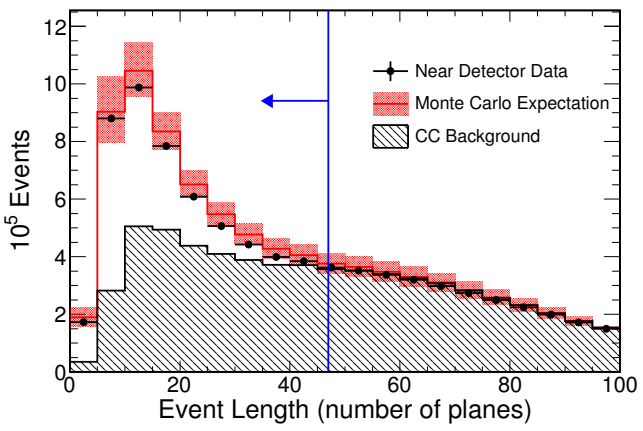

(a)

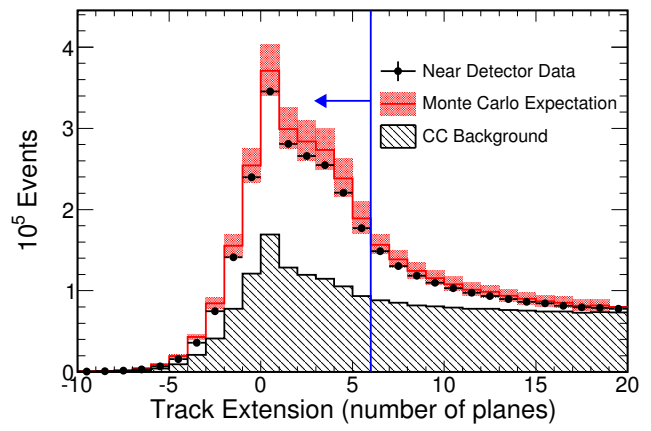

(b)

Figure 4.22: Two variables are used for selecting NC events: event length (left) and track extension (right). The Near Detector data, showed in black dots, is compared to the Monte Carlo prediction with systematic error (red shaded band). The CC background is shown in black hatched histogram. Figure taken from [116].

\subsubsection{Improving the neutral-current selection}

Inspired by the success of the $\mathrm{kNN}$ classifier, a multi-variate technique is developed to improve the NC selection [117]. $80 \%$ of the selected NC events come from the with-track sample; the remaining $20 \%$ consists of events with no track. By applying the k-nearest neighbors algorithm with four input variables, the signal efficiency of even-with-track sample can be improved by more than $12 \%$ while keeping the signal purity at the same level to the current selection. The four input variables are: (a) event length, (b) track extension, (c) maximum consecutive plane, and (d) mean pulse height per strip in track. Figure 4.23 shows the distributions of these four variables for the Monte Carlo simulated NC events (signal) and CC events (background) in the Near Detector. Distribution of NC/CC separation parameters is shown in Figure 4.24. 

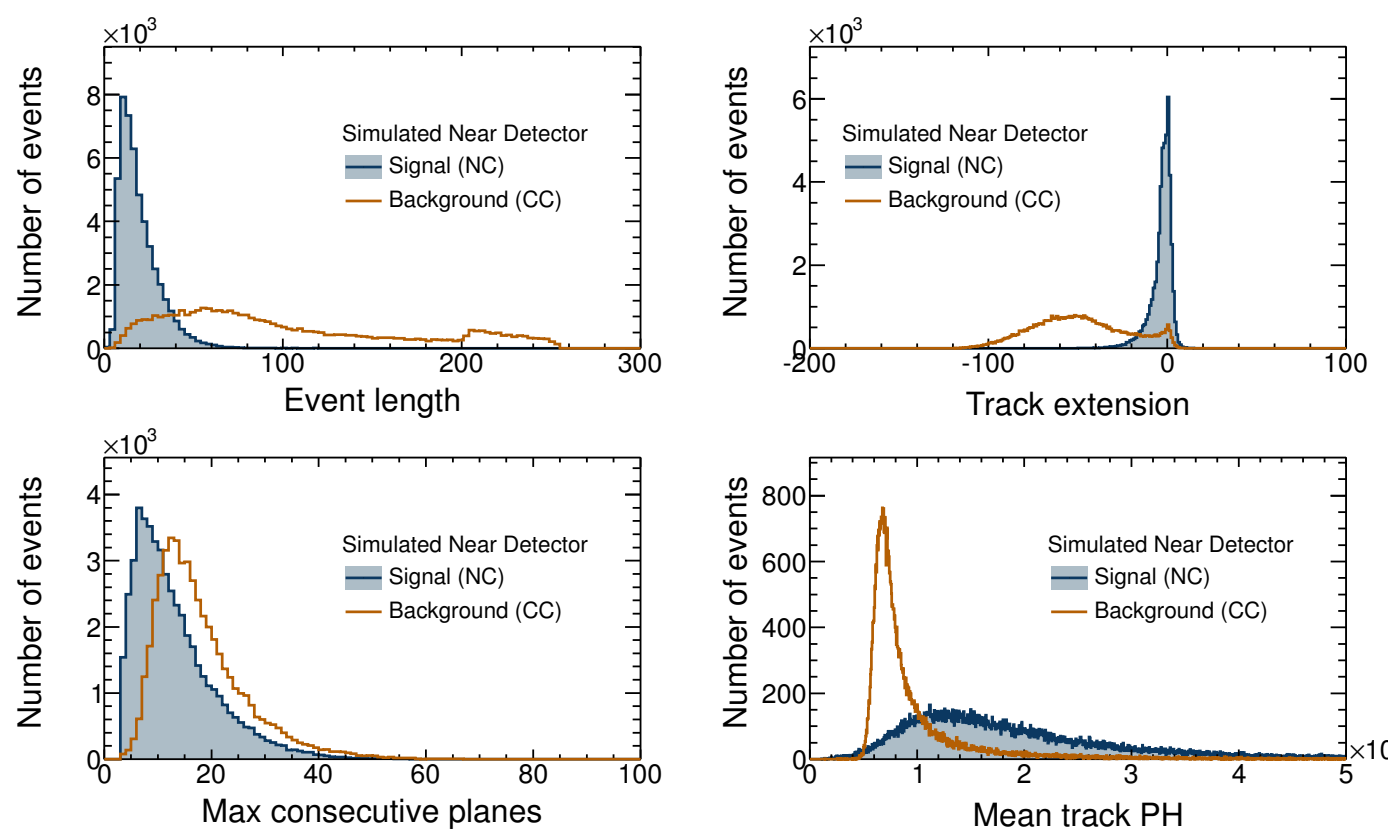

Figure 4.23: Four input variables are used for a new neutral-current event selection in the event-with-track sample. NC events (signal) are shown in the blue histogram and CC events (background) are shown in the orange histogram.

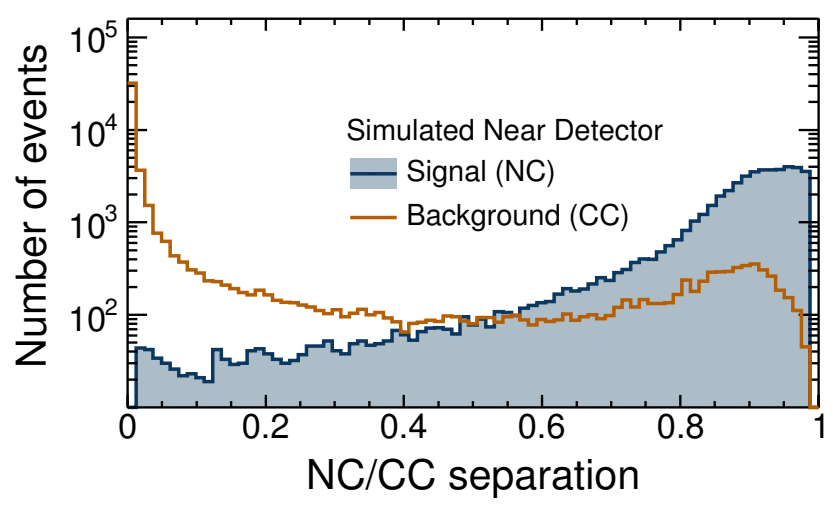

Figure 4.24: Distribution of "NC/CC separation" parameter as the result of mapping four input variables. 
By making a cut on this parameter to keep signal purity in the same order with the current selector, we see a significant improvement in the signal efficiency. Table 4.5 shows the comparison of total signal efficiency and purity between the current selector and new selector.

\begin{tabular}{|l|c|c|}
\hline Performance & Current selector & New selector \\
\hline Signal efficiency & 72.9 & 85.8 \\
\hline Signal purity & 67.7 & 67.8 \\
\hline
\end{tabular}

Table 4.5: Comparison of the signal efficiency and purity between the current selector and the new s elector for the event-with-track sample.
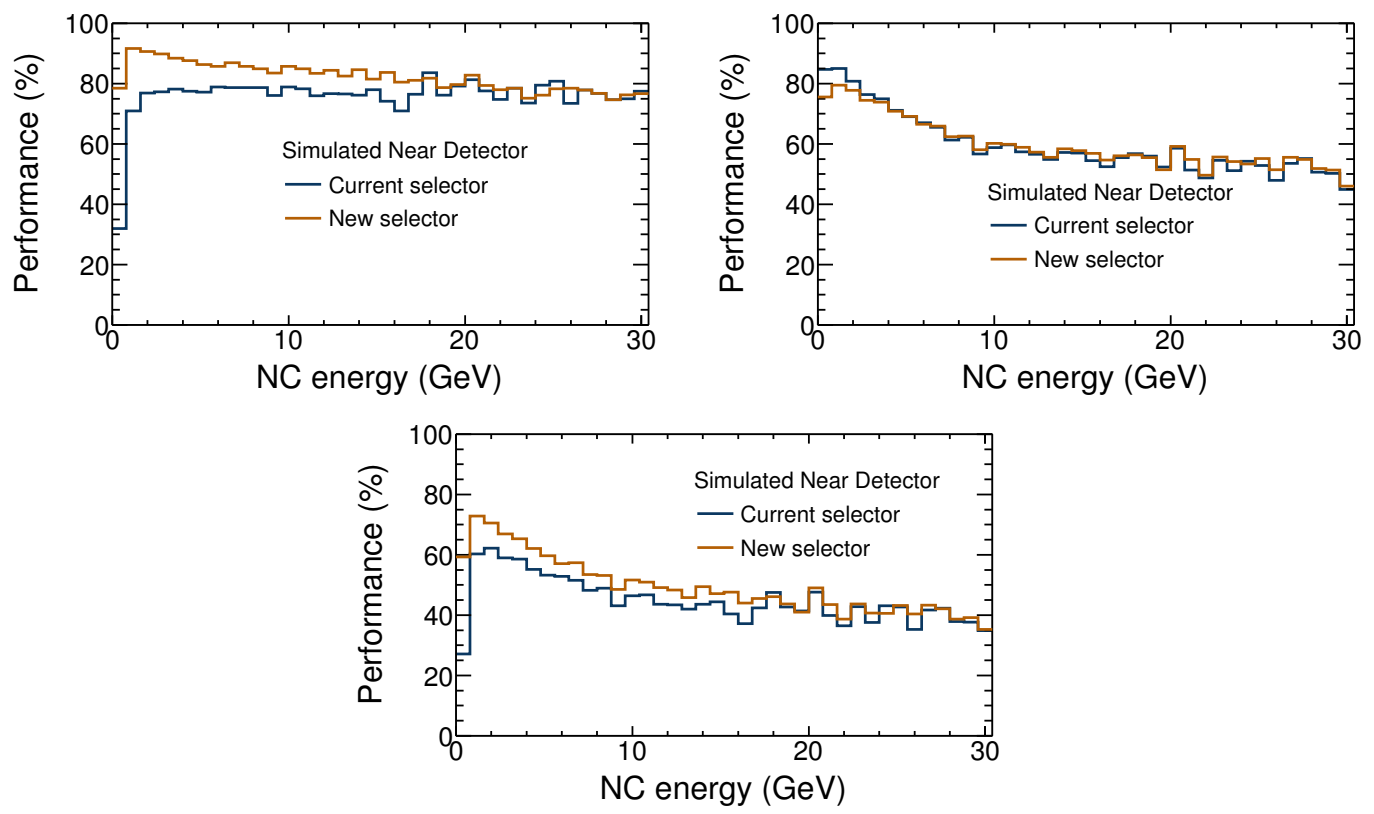

Figure 4.25: Comparison of the signal efficiency (left), purity (right), and product of efficiency and purity (bottom) for the event-with-track sample between the new selector and current selector. 
Figure 4.25 shows the performance comparison between the current selector and the new selector. For the event-with-no-track sample, the multi-variate technique was used, but no notable improvement was observed [117]. In combination, the PID cut for events with tracks are applied and the original cut for the events with no track is held back as the current selector. The signal purity of the new selector does not change but its efficiency increases by more than $9 \%$ ( $80 \%$ statistics of $12 \%$ improvement in the event-with-track sample) in comparison to the current selection.

This is a very preliminary study for selecting the neutral-current events with the multi-variate technique. Some other distinguishable features can also be studied while the systematic uncertainties need to be considered carefully.

\subsection{Summary of event classification}

In this chapter, two new selections for selecting $\bar{\nu}_{\mu}$ charged-current events and neutral-current events were developed for better performance. The improvement of new $\bar{\nu}_{\mu}$-CC event selection in the $\bar{\nu}_{\mu}$-beam mode matches an approximate $10 \%$ statistical increase of current data. A new multivariatetechnique approach for selecting the neutral-current events yielded $9 \%$ increase of the signal efficiency while keep the signal purity at the same level as the current selector. The study of event classification aims to increase the sensitivity to the oscillation parameters, which could be further improved by implementing a new hadronic shower energy estimator that is the subject of Chapter 5 . 


\section{Chapter 5}

\section{The hadronic shower energy estimator}

This chapter introduces the adoption of supervised multivariate algorithms to estimate the hadron shower energy. In previous analyses, the total recorded hits, in which the track hits are excluded, were employed to measure the hadronic shower energy. We call this the calorimetric approach. This approach yields a poor shower energy resolution due to the uncertainties in energy deposition on the steel planes. The multivariate technique is developed to improve the shower energy estimation by using the topological and dynamic information about the showers. The improvement of neutrino energy resolution, in turn, increases the sensitivity of MINOS analysis to determine oscillation parameters.

\subsection{Motivation for hadronic shower energy estimator}

The precision measurement of oscillation parameters, measured via the disappearance of charged-current (CC) $\nu_{\mu}$ events, depends dramatically on the energy resolution of selected events. Providing that the energy estimator is perfect, i.e reconstructed energy for each Monte Carlo simulated event is hypothetically equal to its true energy, we find a substantial sensitivity im- 
provement can be achieved. Figure 5.1 depicts the sensitivity achieved by the perfect energy estimator in comparison to the sensitivity obtained by the standard energy reconstruction. The sensitivity of the perfect energy estimator is matched to more than $40 \%$ statistical increase of the current data.

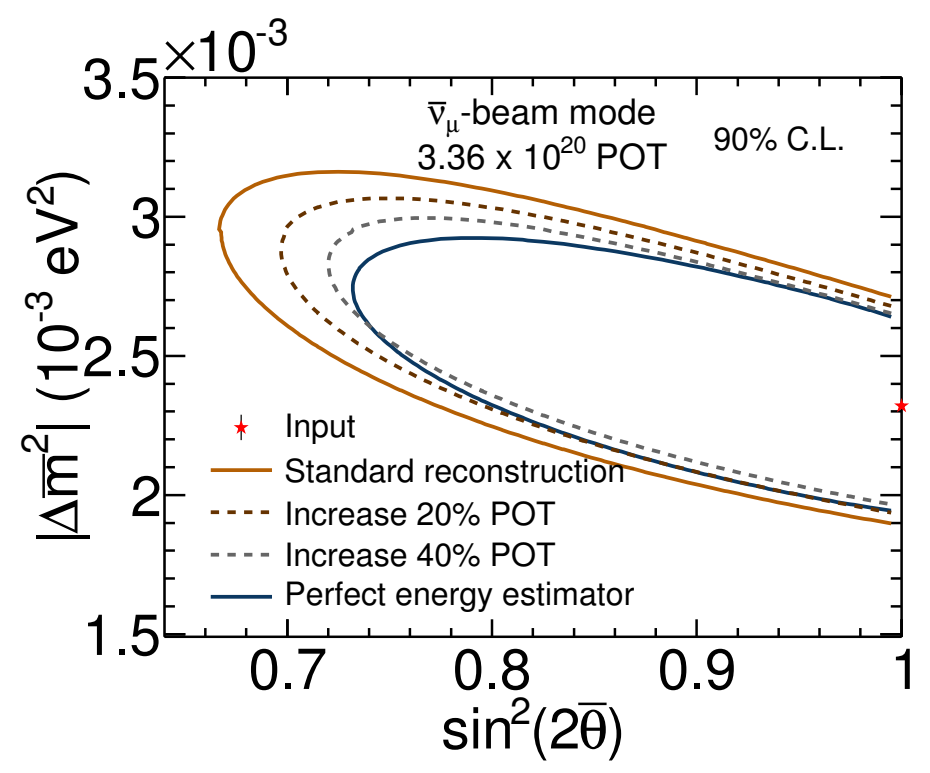

Figure 5.1: Comparison of sensitivity contours with the standard energy reconstruction (orange) and the perfect energy estimator (blue). The sensitivity gained by the perfect energy estimator is matched more than $40 \%$ statistically increase of the current data (dashed grey).

The total energy of each $\nu_{\mu}$-CC event is essentially the sum of muon track energy and hadronic shower energy. Figure 5.2 shows the resolution of calorimetric shower energy and track energy measured by using the muon track range in the Far Detector. Clearly, the reconstructed calorimetric shower energy resolution, $\sim 56 \%$ of $\sqrt{E_{\text {shw }}}$, is much worse than the reconstructed track energy, $\sim 5.1 \%$ of $\sqrt{E_{\text {trk }}}$. 


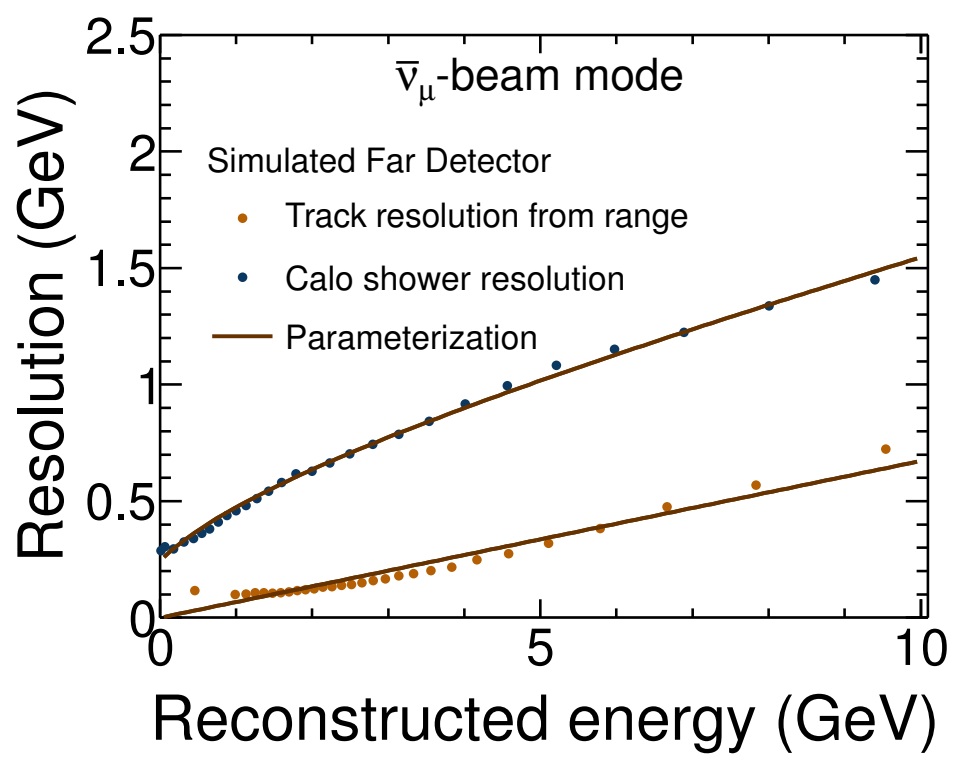

Figure 5.2: The simulated Far Detector calorimetric shower (orange) and track (blue) energy resolution measured by using the muon track range in the detector. The parameterizations of resolutions are also presented.

The total energy of each $\nu_{\mu}$-CC event is roughly divided equally into track energy and shower energy. Thus, event energy resolution, which is a quadratic sum of energy resolutions of shower $\left(\sigma_{\text {shw }}\right)$ and track $\left(\sigma_{\text {trk }}\right)$ :

$$
\sigma_{\mathrm{tot}}=\sqrt{\sigma_{\mathrm{shw}}^{2}+\sigma_{\mathrm{trk}}^{2}},
$$

is dominated by the shower energy resolution. This means an improvement of the shower energy resolution would have a more substantial impact on the oscillation sensitivity than that of the track energy resolution. 


\subsection{Shower characteristics}

Basically two main categories of showers are recorded in the MINOS detectors: electromagnetic showers and hadronic showers. Their distinctive features are briefly described as follows:

\section{Electromagnetic showers}

The activities of this type of shower are well understood. Electrons and positrons lose their energy via ionization and radiation processes. The former process dominates at low energy while the latter governs at high energy. At energies of $1 \mathrm{GeV}$ or higher, the electrons and positrons lose most of their energy via the Bremsstrahlung radiation. The energetic emitted photons, in turn, convert into $e^{+} e^{-}$pairs, which gives another electron and positron. The multiplication of shower particles (electrons, positrons and gamma ray) reaches a maximum at a certain depth inside the absorber of the detector, and gradually decreases beyond that depth ${ }^{1}$. Because of the particle multiplication, the longitudinal development of electromagnetic showers is relatively short. The resolution of electromagnetic showers measured by the Calibration Detector is $\sim 21 \%$ of $\sqrt{E_{\text {shw }}}[118]$.

\footnotetext{
${ }^{1}$ The depth of shower maximum increases logarithmically with the energy of the incoming electrons
} 


\section{Hadronic showers}

Compared with the electromagnetic shower, hadronic showers are more chaotic due to the multi-particle production and particle emission resulting from the decay of excited nuclei. Also, because of a fairly large number of produced neutral pions, the hadronic shower typically includes a component of electromagnetic showering development. A large portion of energy is deposited towards the end of a hadronic shower while an electromagnetic shower develops concentratedly in the first portion of the shower. Hadronic showers also exhibit a more transverse scattering than electromagnetic showers. The resolution of

hadronic showers measured by Calibration Detector is $\sim 56 \%$ of $\sqrt{E_{\mathrm{shw}}}[119]$, which is worse than that of electromagnetic shower resolution. The limitation of hadronic shower resolution is due to the fluctuation of $\pi^{0}$ production and energy leakage (a large fraction of shower is not appeared as detectable signals).

\subsection{Regression analysis for shower energy estimator}

Using a multivariate algorithm (MVA) technique to estimate the shower energy is a typical application of a regression analysis, which estimates the value of a desired single variable (or vector) in terms of other input variables. In the calorimetric approach, the sum of all shower-deposited hits is simply assigned as the reconstructed shower energy. The calorimetric shower energy from this approach yields a poor resolution because of the uncertainties in energy deposition on the steel plane and information loss due to the event reconstruction. The regression analysis, on the other hand, utilizes the MVA 
techniques to regain these losses by taking advantage of a broader range of shower features. This can be done by employing the Monte Carlo simulation, in which the true shower energy of each event is known. The regression process can be summarized by the following steps:

1. The simulated Monte Carlo sample forms a training set, in which each event is described by selected measurable variables and its true shower energy is stored as a target value.

2. A training process, based on correlations among selected measurable variables, is implemented in the training set to find the rules or regression functions that predict target values for each event. The MVA algorithms are applied in this step to build the relationship between the multiple variables and the target in the training set.

3. These rules or regression functions are then applied for each observed event (either data or simulated Monte Carlo sample) to estimate its shower energy.

Selected measurable variables and algorithms play vital roles for the success of this kind of data mining. These variables must be correlated with the target (i.e., true shower energy) while the algorithm must take the advantage of these correlations as much as possible. 


\section{4 kNN shower energy estimator}

The inspiration for using a k-nearest neighbors (kNN) estimator for measuring the shower energy comes from the successful implementation of this estimator for muon track identification [120]. The kNN shower energy estimator is an example of a regression analysis (discussed in Section 5.3), in which the shower energy plays a role as target and the regression functions are determined by the kNN algorithm.

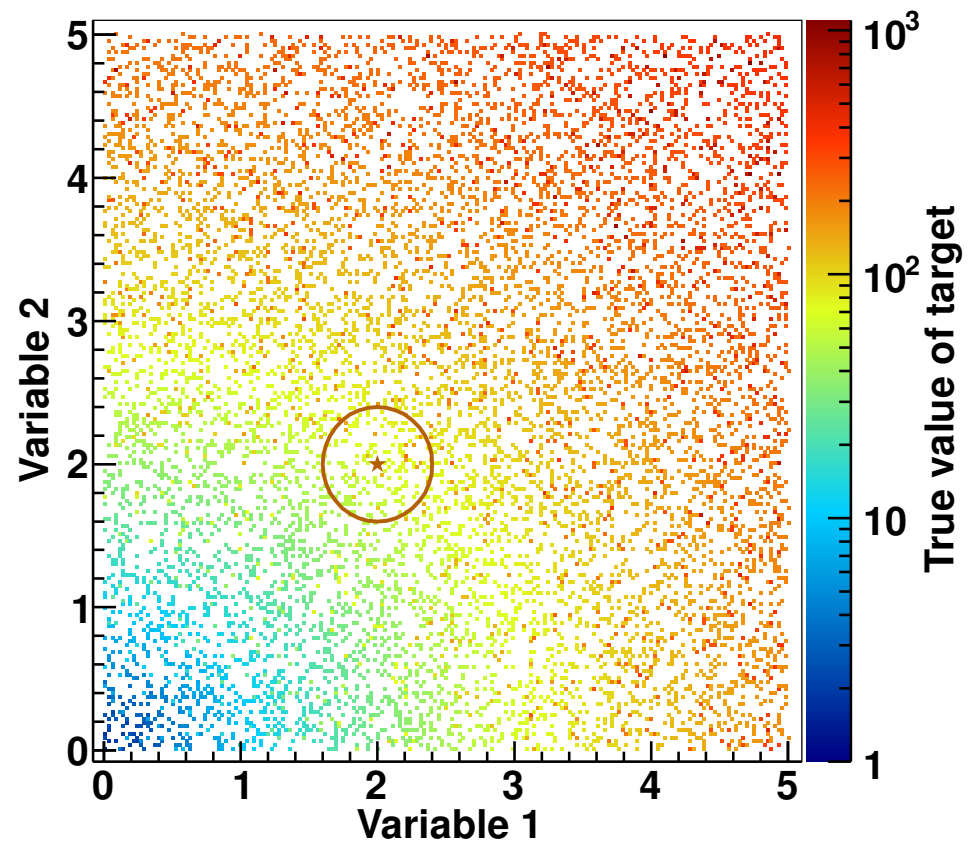

Figure 5.3: Graphic view of employing the kNN shower energy estimator in two-dimensional space. The z-axis (in color palette) present the true target value of events. Two input variables cluster the training set into multiple regions. For each observed event (star point), the mean of true target value of its nearest neighbors inside the circle is assigned as its target value. 
Figure 5.3 illustrates the implementation of the $\mathrm{kNN}$ algorithm to estimate the shower energy for an observed event in two-dimensional space. The large statistical sample of a simulated Monte Carlo are employed to build a training set, in which each event is recorded with a number of input variable values and the true shower energy (true value of target). The correlation between the input variables and true shower energy facilitates a division of the training set into a number of regions, in which the true shower energy of events are approximately the same. For an observed event, a fixed number $(\mathrm{k})$ of its nearest neighbors in the training set are found. The mean of the true shower energy of its nearest neighbors is then assigned as the shower energy for that observed event.

To perform the kNN shower estimator in MINOS, a list of potential variables, which characterize the hadronic showers, are considered. For each combination of these variables, the same number of nearest neighbors are kept and the estimator performance is judged by looking at the sensitivity to oscillation parameters gained by the output of estimator. The judgement criteria is based on the error achieved on the $\Delta m^{2}$, assuming the maximal mixing angle $(\sin 2 \theta=1)$. The estimator with the best performance based on this criteria is with the three input variable method [120]: (i) the calorimetric shower energy in the first two showers, (ii) the deweighted energy ${ }^{2}$ within 1 meter of the track vertex, and (iii) the number of planes in the primary shower.

\footnotetext{
${ }^{2}$ Deweighted energy is an energy estimator in which the energy deposited in each strip is raised to a energy-depended power between zero and one before they are summed.
} 
Figure 5.4 shows the distributions of these three input variables in comparison between the data and Monte Carlo simulation at the Near Detector. These are plotted with the selected $\bar{\nu}_{\mu}$-CC events in the $\bar{\nu}_{\mu}$-beam mode. The number of nearest neighbors is optimized in the same way, and $k=400$ was ultimately chosen.
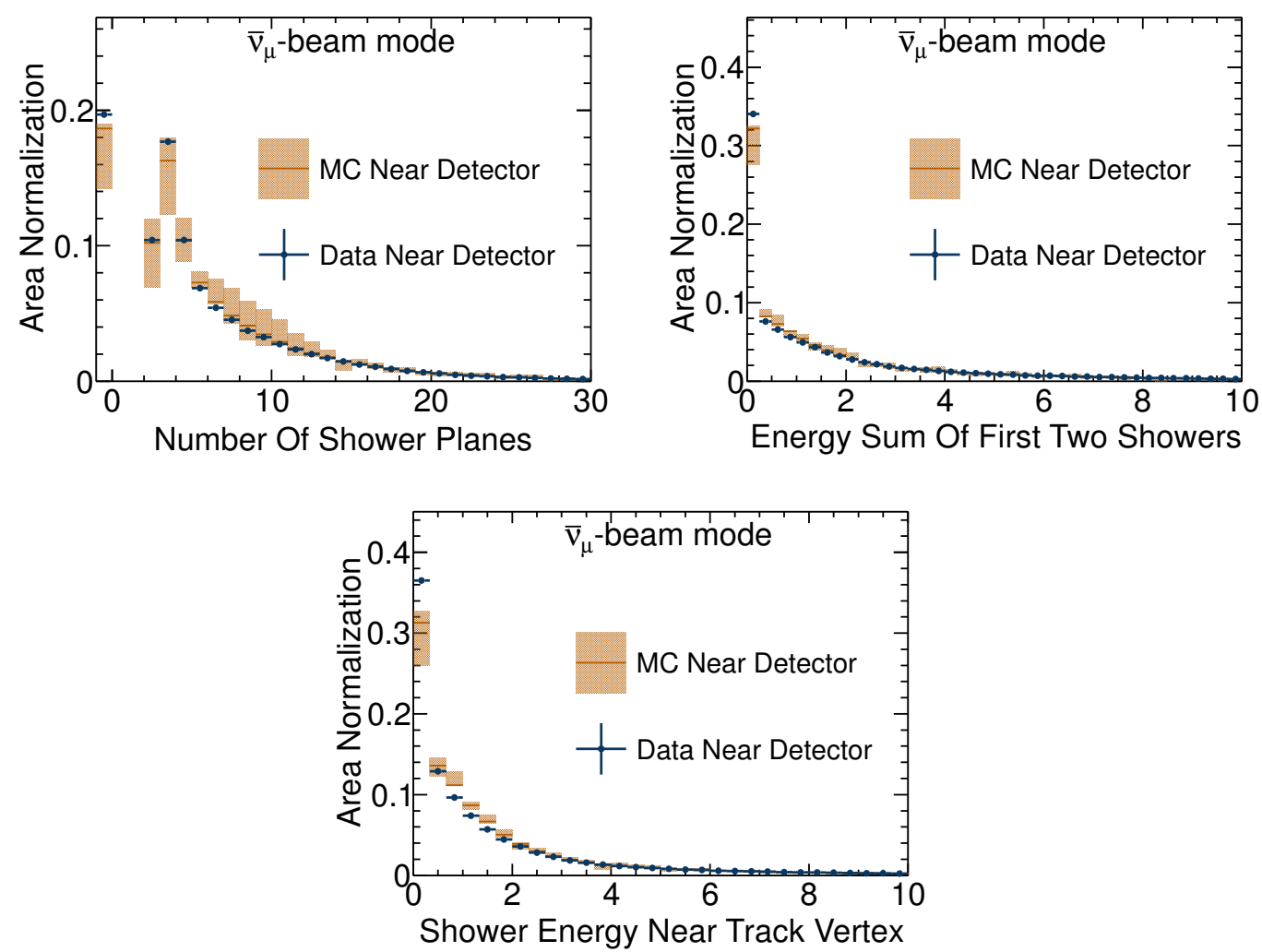

Figure 5.4: Distributions of three input variables of $\mathrm{kNN}$ estimator in comparison between the data (black dots) and Monte Carlo simulation (orange histogram) at the Near Detector: (i) number of shower planes (top left), (ii) energy sum of first two showers (top right), and (iii) shower energy near track vertex (bottom). These are plotted for $\bar{\nu}_{\mu}$-CC events in $\bar{\nu}_{\mu}$-beam mode. 
Taking the average of the true shower energies of 400 nearest neighbors for each observed event might lead to a bias in the shower energy estimates. For example, an observed event has low true shower energy but picks up the nearest neighbors of true higher energies, it yields an overestimation and vice versa. This situation is handled by applying an energy correction function to make sure that the mean value of shower energy estimated by the kNN estimator agrees with the true shower energy in every range of energy. To do that, a profile of shower $E_{\mathrm{kNN}} / E_{\text {true }}\left(E_{\mathrm{true}}\right.$ is the true shower energy and $E_{\mathrm{kNN}}$ is the raw output of kNN estimator) is plotted as the function of $E_{\text {true }}$. A $7^{\text {th }}$ polynomial function, $f\left(E_{\text {true }}\right)$, is then used to fit this profile.

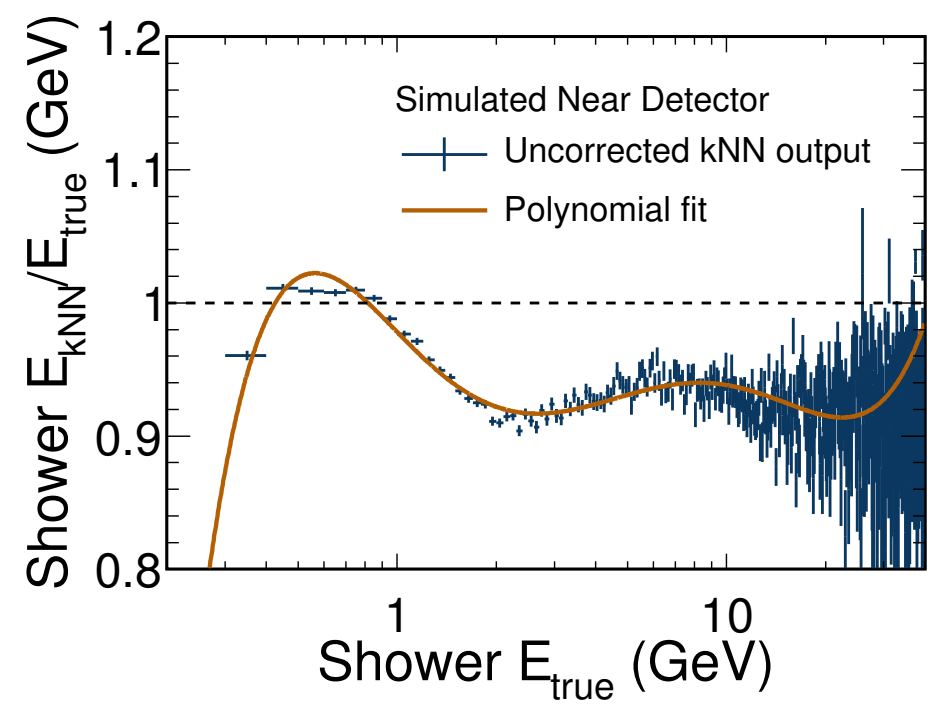

Figure 5.5: The profile of shower $E_{\mathrm{kNN}} / E_{\text {true }}$ as the function of $E_{\text {true }}$ (blue dots) is fitted with a polynomial fit (orange line). The fitted function then is applied to tune the $E_{\mathrm{kNN}}$ into a corrected shower energy estimate for each event. This is plotted with selected $\bar{\nu}_{\mu}$-CC events of simulated Near Detector sample in the $\bar{\nu}_{\mu}$-beam mode. 
The fitted function is applied to tune the $E_{\mathrm{kNN}}$ of each event into a corrected shower energy, which is finally assigned as the event shower energy. Figure 5.5 shows an illustration of energy correction for tuning the kNN shower energy estimator with the simulated sample at the Near Detector.

The performance of the kNN shower estimator has been demonstrated to be very powerful in improving the shower energy resolution and sensitivity to oscillation parameters [120]. This technique was initially implemented for estimating shower energy of $\nu_{\mu}$-CC events in the $\nu_{\mu}$-beam mode, and the training set is created from the Monte Carlo sample for the $\nu_{\mu}$-beam mode. To apply this technique for estimating of $\bar{\nu}_{\mu}$-CC events, a retraining process is necessary. The reason is that these two types of interactions are not the same. The $\nu_{\mu}$-CC events exchange $W^{-}$while the $\bar{\nu}_{\mu}$-CC events exchange $W^{+}$ during the interactions. Their inelasticity, cross-section and energy range are also different. As a consequence, their true shower energy, as well as their measurable shower features (input variables for the $\mathrm{kNN}$ estimator), are not the same. Figure 5.6 shows the profile of shower $E_{\mathrm{kNN}} / E_{\text {true }}$ as the function of shower $E_{\text {true }}$ for selected $\bar{\nu}_{\mu}$-CC events in the $\bar{\nu}_{\mu}$-beam mode, which are processed with a training set of selected $\nu_{\mu}$-CC events in the $\nu_{\mu}$-beam mode. The deviation of this profile away from unit indicates that the kNN shower energy estimator does not perform correctly and needs to be retrained. A new training set, which consists of selected $\bar{\nu}_{\mu}$-CC events in the $\bar{\nu}_{\mu}$-beam mode, is created for estimating the shower energy of $\bar{\nu}_{\mu}$-CC events. Figure 5.7 shows the profile of shower $E_{\mathrm{kNN}} / E_{\text {true }}$ as the function of shower $E_{\text {true }}$ for selected 
$\bar{\nu}_{\mu}$-CC events in the $\bar{\nu}_{\mu}$-beam mode with new training set. Profiles with an applied energy correction agree with unit. This means the mean value of $\mathrm{kNN}$ shower energy agrees with the true shower energy in every range of energy.
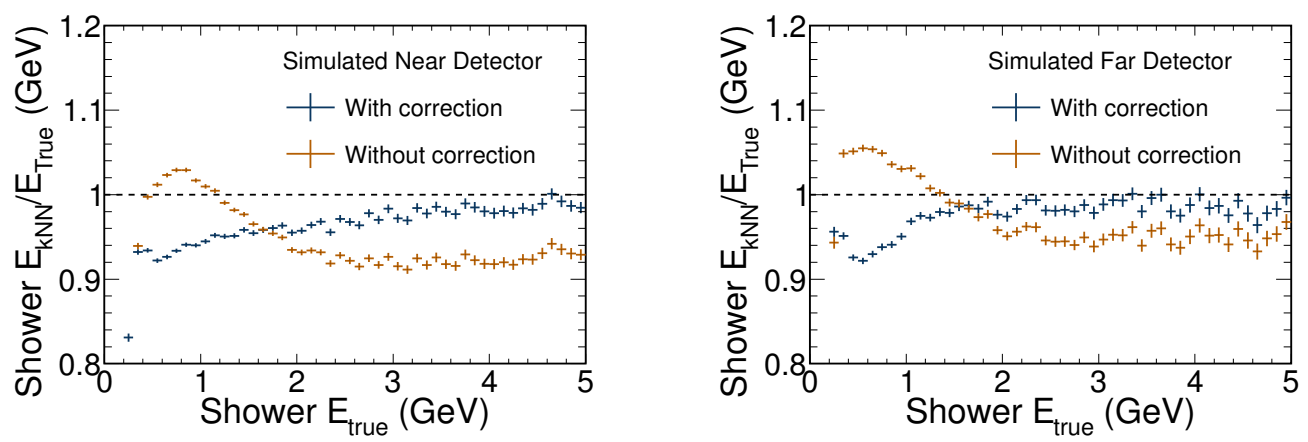

Figure 5.6: The profile of shower $E_{\mathrm{kNN}} / E_{\text {true }}$ as the function of shower $E_{\text {true }}$ of selected $\bar{\nu}_{\mu}$-CC events in the $\bar{\nu}_{\mu}$-beam mode, processed with training set of selected $\nu_{\mu}$-CC events in the $\nu_{\mu}$-beam mode. The profiles for simulated Near Detector (left) and Far Detector (right) sample are plotted with (blue) and without (orange) applying correction function.
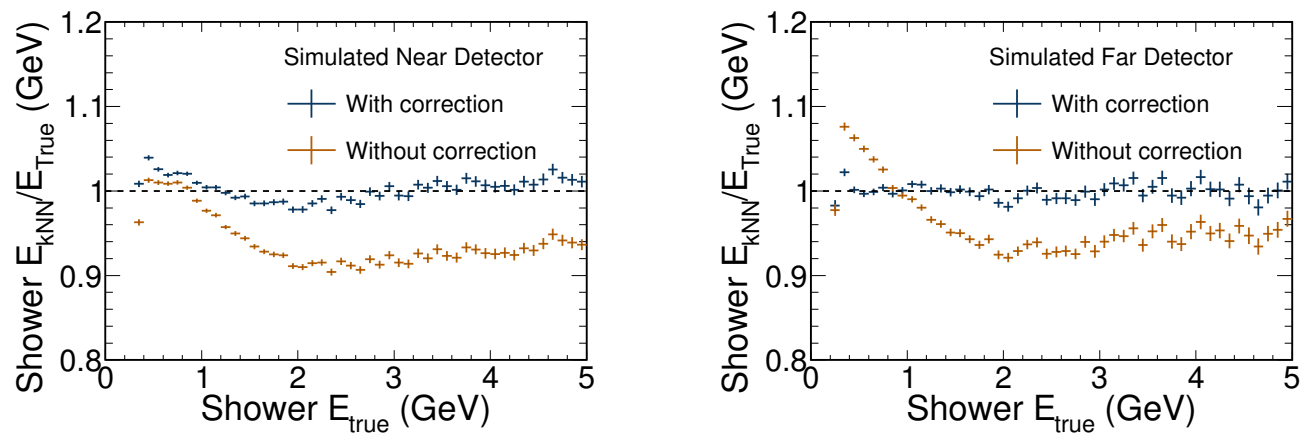

Figure 5.7: The profile of shower $E_{\mathrm{kNN}} / E_{\text {true }}$ as the function of shower $E_{\text {true }}$ of selected $\bar{\nu}_{\mu}$-CC events in the $\bar{\nu}_{\mu}$-beam mode with new training set. The profiles for simulated Near Detector (left) and Far Detector (right) sample are plotted with (blue) and without (orange) applying correction function. 
The improvement of the kNN shower energy estimator in comparison to the calorimetric shower energy is demonstrated in Figure 5.8. The distributions of kNN shower energy show sharper peaks than those of calorimetric shower energy. In other words, the shower energy resolution from the kNN estimator is better than its calorimetric approach.

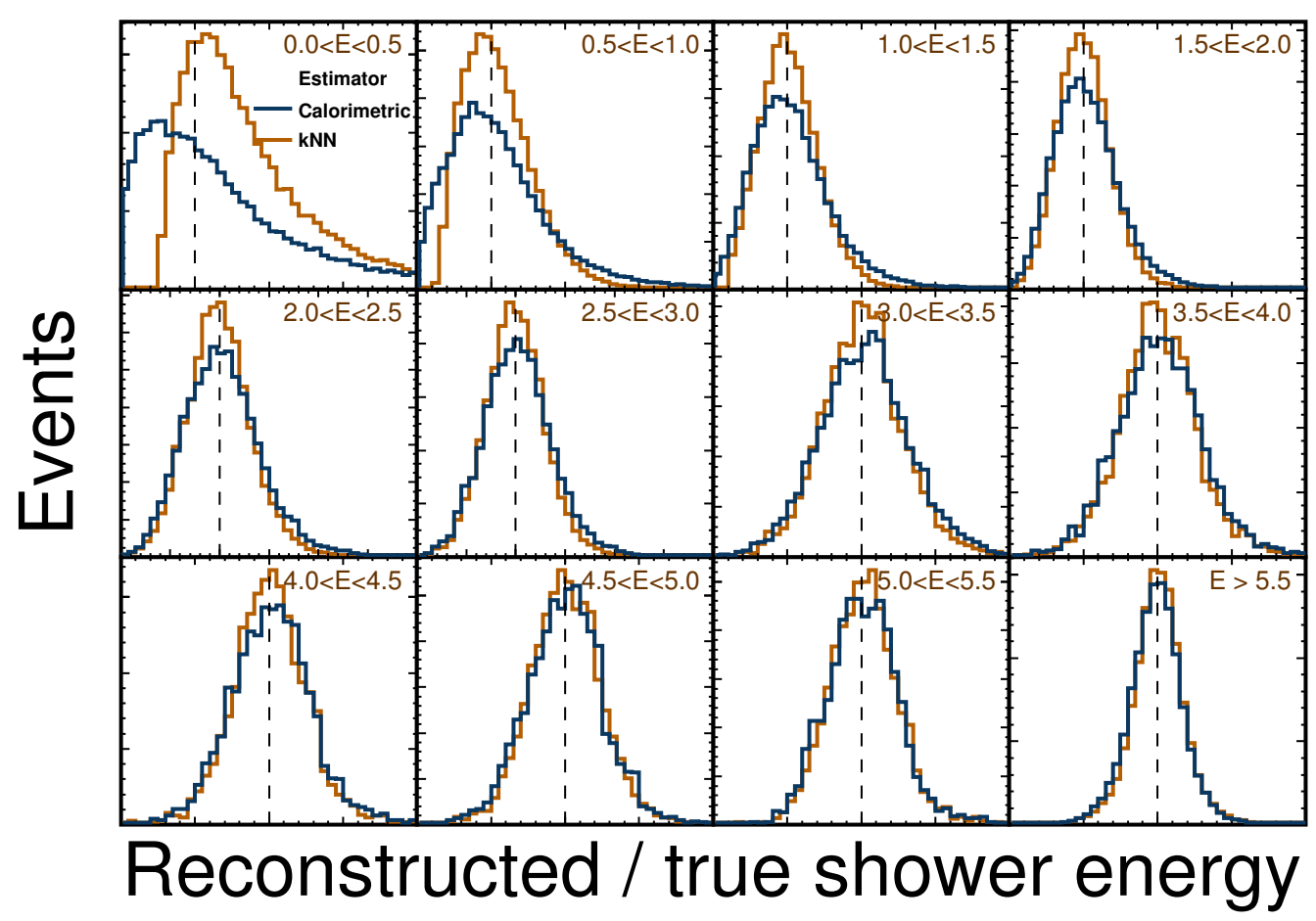

Figure 5.8: Distribution of shower $E_{\text {reco }} / E_{\text {true }}$ for different ranges of shower $E_{\text {true }}$ at the Far Detector. The blue and the orange histograms are with the calorimetric and kNN shower estimators respectively. The dotted lines mark $E_{\text {reco }} / E_{\text {true }}=1$. 
To quantify the improvement of kNN shower energy resolution, we define the factor of shower energy resolution improvement, $R$, as follows:

$$
R=\left|\frac{E_{\text {calo }}}{E_{\text {true }}}-1\right|-\left|\frac{E_{\mathrm{kNN}}}{E_{\text {true }}}-1\right|
$$

where $E_{\text {calo }}$ is the calorimetric shower energy. This factor represents how close the $\mathrm{kNN}$ shower energy is to the truth shower energy in comparison to the calorimetric shower energy. Figure 5.9 shows that an approximate $30 \%$ improvement factor at low energies is achieved by the performance of the $\mathrm{kNN}$ shower estimator. Figure 5.10 shows the comparison of fractional resolution, $\sigma_{E} / E$, of shower energy obtained by $\mathrm{kNN}$ and calorimetric estimators. The improvement of shower energy resolution leads to better total energy resolution of the event, which increases the sensitivity to the precision estimation of oscillation parameters. Figure 5.11 depicts the improvement of sensitivity to oscillation parameters achieved by employing the kNN shower energy estimator. The sensitivity gained by this estimator matches a $10 \%$ statistical increase of the current data. 

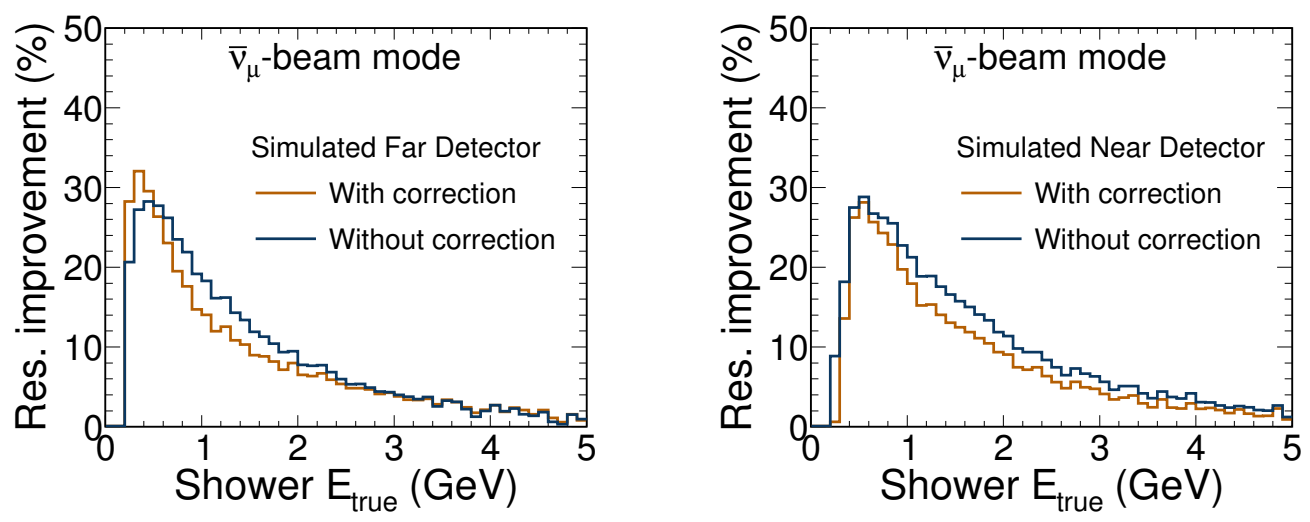

Figure 5.9: The resolution improvement as a function of true shower energy for the simulated Far Detector (left) and Near Detector (right). The y-axis is the improvement $R$ which is defined in Eq. (5.2). The blue and orange histograms are with kNN shower energy before and after correction respectively.

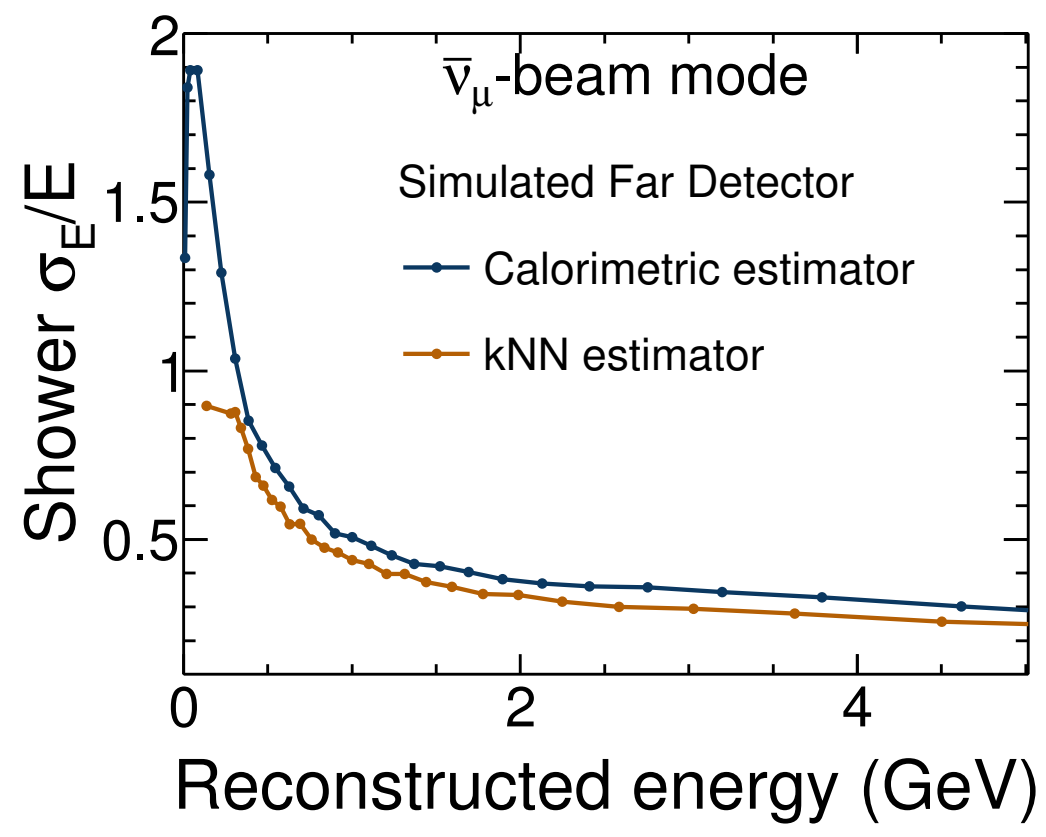

Figure 5.10: The shower energy resolution with the calorimetric and $\mathrm{kNN}$ shower estimators plotted as the function of corresponding shower energy. 

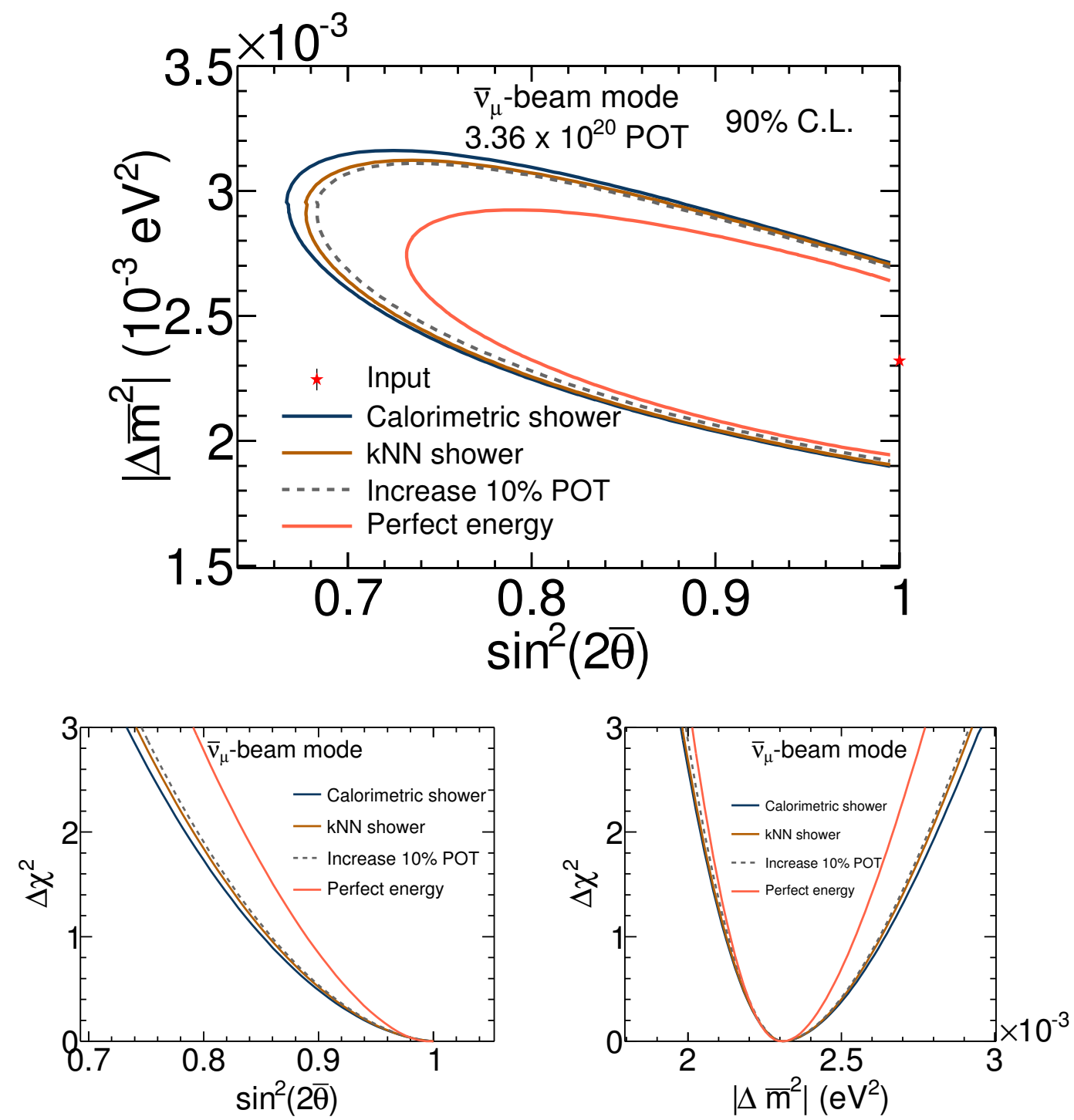

Figure 5.11: The sensitivity contours (top) with different shower estimators, $\mathrm{kNN}$ (blue), calorimetric (orange), perfect energy (red) and calorimetric with $10 \%$ statistical increase of current data, are compared. Their projections on mixing angle, $\sin ^{2}(2 \bar{\theta})$ and on mass-squared splitting, $\left|\Delta \bar{m}^{2}\right|$ are shown respectively on the bottom left and on the bottom right. 


\subsection{Systematic uncertainties of the kNN shower energy estimator}

The performance of the kNN shower energy estimator depends dramatically on the Monte Carlo sample, which is used to build the training set. The output of this estimator might be sensitive to the mismodeling of the hadronic showering process. Thus, an investigation of the systematic errors caused by this estimator is necessary. Three main sources of systematic errors for the kNN shower energy estimator are highlighted as follows:

- Detector calibration: The uncertainty is estimated as the maximum discrepancy between data and Monte Carlo simulation for the response of the Calibration Detector to single particles. This uncertainty is reported at $5.7 \%[119]$.

- Hadronization modeling: The uncertainty is due to the unknown particle content of the final state right after neutrinos interact with the nuclei in the detectors.

- Intranuclear rescattering: The uncertainty comes from the re-scattering of produced hadrons before they have been observed by the detectors.

The approach for estimating the systematic error is similar to what was used for the hadronic shower energy in previous study [120]. The backbone of this approach is comparing the shower energy in the nominal Monte Carlo sample to the number of tuned Monte Carlo samples, which are modeled or shifted 
respectively for systematic errors. The profile of shower ratio $E_{\mathrm{kNN}} / E_{\text {true }}$ as the function of shower $E_{\text {true }}$ are chosen as the criteria to judge systematic uncertainty. The total systematic error is calculated from all systematics.

\subsubsection{Uncertainties due to detector calibration}

Two of the three input variables of $\mathrm{kNN}$ shower energy estimator depend on energy calibration. To investigate the systematic error of energy calibration, a shifted sample, in which these two variables are scaled by the absolute calibration scale, is produced. The kNN shower energy of events in the shifted sample is re-evaluated. The deviation of kNN shower energy in the shifted sample from the nominal one is shown in Figure 5.12. On average, the shifts of kNN shower energy is smaller than the absolute calibration scale in every range of true shower energy.

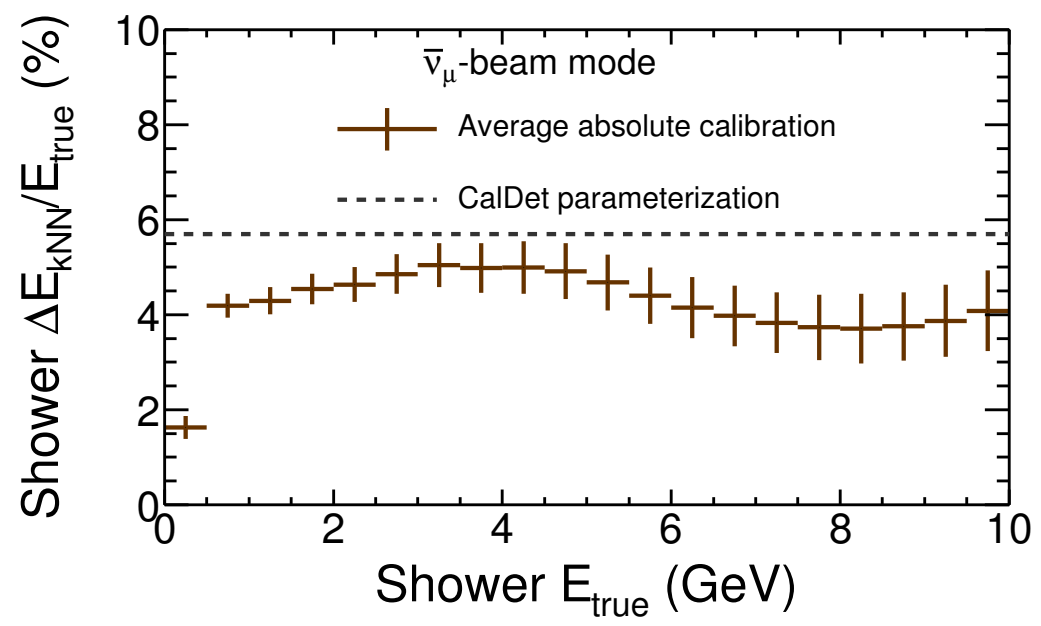

Figure 5.12: Average shift on the kNN shower energy due to the shifts of energy calibration. This compared to $5.7 \%$ uncertainty of the detector calibration. 


\subsubsection{Uncertainties due to hadronization modeling}

The "AGKY" model, described in more detail in [121], is implemented to model the hadronization in the MINOS detectors. However the particle content of the final state in the neutrino interactions is not always well-known, especially in the deep inelastic scattering. To evaluate the systematic uncertainty of hadronization modeling on the output of $\mathrm{kNN}$ shower energy estimator, a number of special Monte Carlo samples, which are constrained by external data, are generated to evaluate the sources of uncertainty. Eight sources of uncertainty from hadronization modeling are listed below:

1. Pion/nucleon absorption: The uncertainties in probabilities of absorbing pions and nucleons are respectively found to be $\pm 30 \%$ and $\pm 20 \%$ [122]. Two new $\pm 1 \sigma$ Monte Carlo samples are produced based on correlated shifts in these two probabilities.

2. Baryon $x_{F}$ selection: From the experimental measurement of baryon $x_{F}$ distribution [121], the baryons in the final state, at the hadronic center of mass, are produced inside the backward hemisphere which is opposite of the direction of momentum transfer. This is explained by hypothesizing that the neutrino strikes one constituent quark and two remained quarks of hadron are formed into pions inside the forward hemisphere. This results in increasing pion energies and reducing baryon energies when boosting back to the lab frame. This effect is turned off as one model to test the uncertainty of $\mathrm{kNN}$ shower energy estimator. 
3. Formation zone: In the hadronization model, the newly formed hadrons are assumed to propagate without interaction in nucleus within a formation length $l_{f}=\tau c$. In the nominal sample, the formation time is fixed at $\tau=0.342 \mathrm{fm} / \mathrm{c}$. By shifting $\pm 50 \%$ of this formation time, two new $\pm 1 \sigma$ Monte Carlo samples are generated to study the uncertainty of the $\mathrm{kNN}$ shower energy estimator.

4. Intranuke assumption - absorption : All energy from absorbing pions are assumed to transfer to a specific number of nucleons according to the Ransome model [123]. Four nucleons are implemented in the nominal sample. Out of concern for the number of nucleons in which the energy of the absorbed pions are distributed, the number of nucleons are doubled when processing a new sample for studying the systematics.

5. Intranuke assumption - deBroglie ring: The uncertainty is taken into account when considering the effect of quantum mechanics on the interaction where the de Broglie wavelength, $\lambda=h c / p$, increases because of low energy pions. As a result, this enhances the probability of reinteraction. This effect can be mimicked by artificially adjusting the pion size. In the nominal sample, pion size is increased by a fixed amount of $0.5 \lambda[124]$. In order to take this systematics into account, the increased amount of pion size is changed to $\pm 0.6 \lambda$, creating new $\pm 1 \sigma$ samples.

6. Charged/neutral particle correlations: In the "AGKY" model, the total multiplicity of hadrons is accumulated and then split correlatively 
into charged and neutral pions. However, measurement indicates that the charged and neutral pion multiplicities are independent [125]. On account of this measurement, the "AGKY" model is adjusted to loosen the correlation of charged and neutral pion multiplicities.

7. Probability of $\pi^{0}$ production: In the nominal sample of the "AGKY" model, the probability of two $\pi^{0}$ production for neutral meson pairs is $30 \%$, which is constrained from external data. This probability varies by $30 \%$ to $21 \%$ as a ( $-1 \sigma$ sample) and $39 \%$ as a $(+1 \sigma$ sample).

8. Two-body decays: The two-body decays are simulated isotropically in the center of mass. Two models are produced by assuming that all particles are produced either perpendicular (labeled $+1 \sigma$ ) to the direction of momentum transfer or parallel to it (labeled $-1 \sigma$ ).

The percentage shifts of kNN shower energy in special MC samples in comparison to the nominal one are shown in Figure 5.13. 

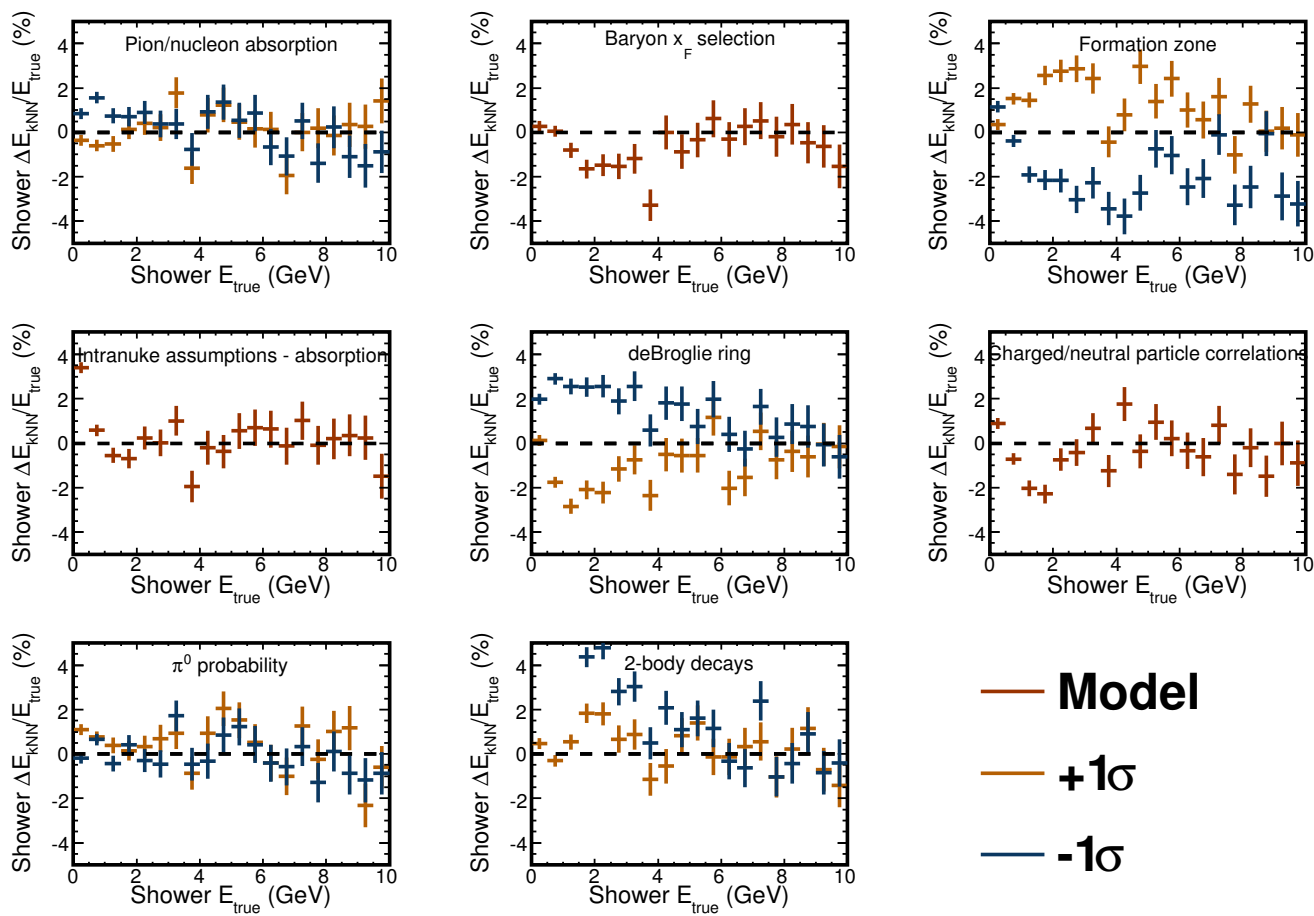

Figure 5.13: The percentage shift in $\mathrm{kNN}$ shower energy for different daikon08 samples as compared to nominal. We have three model samples in the brown histogram and five up/down shifted samples in the orange/blue histograms. These are plotted with selected $\bar{\nu}_{\mu}$-CC events in the $\bar{\nu}_{\mu}$-beam mode.

\subsubsection{Uncertainties due to intranuclear rescattering}

A hadron produced from the neutrino interactions has a high probability to re-scatter with the steel planes before being observed in the detectors. This probability, known as a function of cross-sections and branching ratios, is calculated for each event by using a semi-classical intranuclear cascade model, named Intranuke [126]. To evaluate the effect of this model on the kNN shower energy estimator, the following ten parameters are considered: 
1. Pion charge exchange: shift $\pm 50 \%$ branching ratio of pion charge exchange, which is defined as the probability of charge conversion when pion re-scattering happens.

2. Pion elastic scattering: shift $\pm 10 \%$ branching ratio of pion elastic scattering, which is defined as the probability for pion re-scattering to be elastic.

3. Pion inelastic scattering : shift $\pm 40 \%$ branching ratio of pion inelastic scattering, which is defined as the probability for pion re-scattering to be inelastic.

4. Pion absorption: shift $\pm 30 \%$ probability of pion absorption. This is modeled in a special Monte Carlo sample but in correlation with a shift in pion production probability

5. Pion secondary pion production: shift $\pm 20 \%$ branching ratio of secondary pion production in pion interaction. This is modeled in a special Monte Carlo sample but in correlation with a shift in pion absorption probability.

6. Nucleon knockout: shift $\pm 20 \%$ probability of nucleon knockout reactions.

7. Nucleon secondary pion production: shift $\pm 20 \%$ branching ratio of secondary pion production in nucleon interactions.

8. Pion cross section: shift $\pm 10 \%$ cross-section of pion interactions. 
9. Formation time: shift $\pm 50 \%$ time interval of formation zone. This is overlapped with a special Monte Carlo sample as described above. In calculation of total systematic uncertainty, the shift of $\mathrm{kNN}$ shower energy in the Monte Carlo sample is used.

10. Nucleon cross section: shift $\pm 15 \%$ cross-section of nucleon interactions.
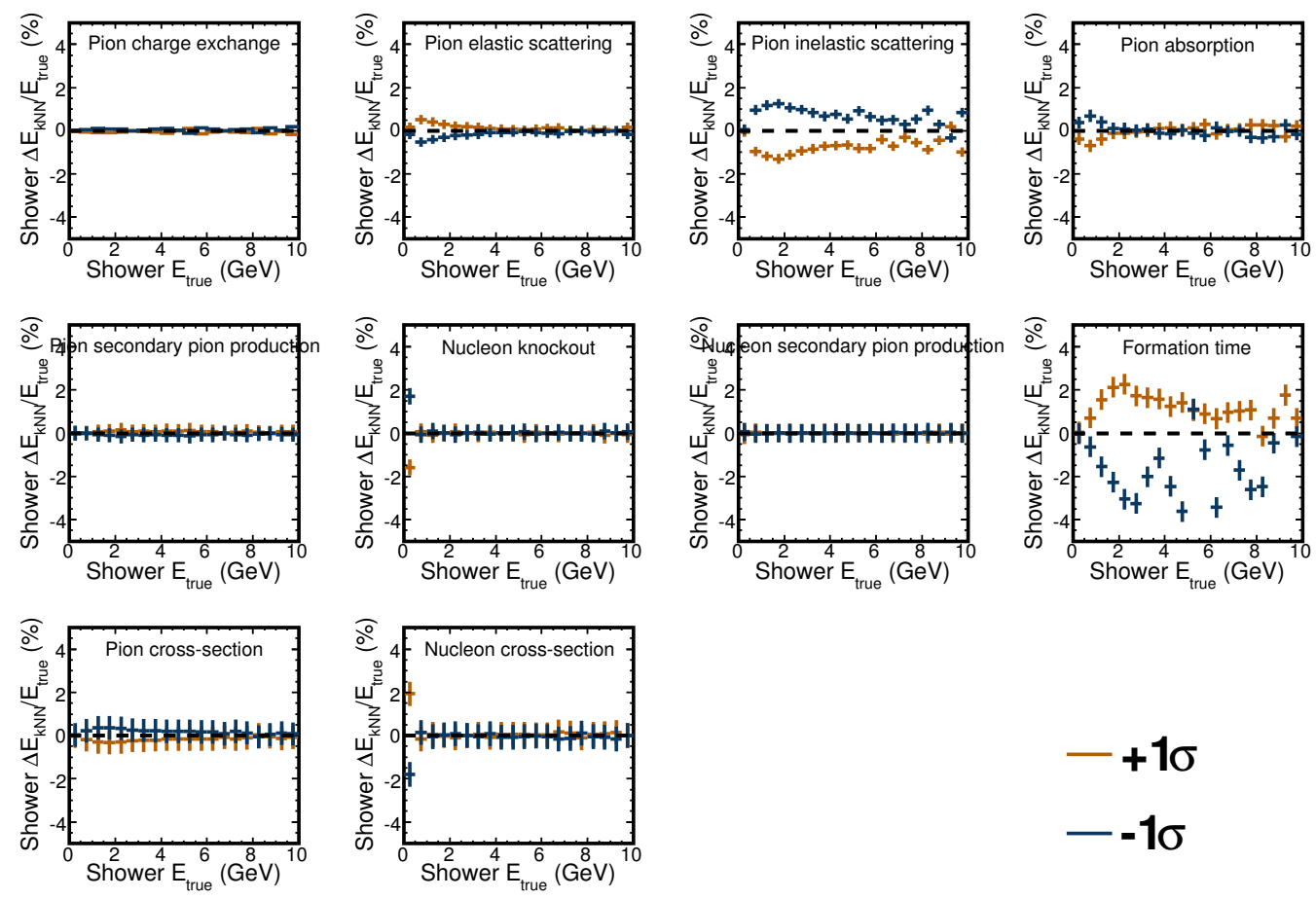

Figure 5.14: The percentage shift in $\mathrm{kNN}$ shower energy under reweighting by different Intranuke systematics as compared to nominal. The orange and the blue histograms are respectively the $+1 \sigma$ and $-1 \sigma$ shifted systematics for each kind of weight. These are plotted with selected $\bar{\nu}_{\mu}$-CC events in the $\bar{\nu}_{\mu}$-beam mode. 


\subsubsection{Total combined systematics}

The overall systematic error of shower energy estimated by kNN algorithm is calculated by taking the quadrature summing of all individual systematics by:

$$
S_{\text {tot }}\left(E_{\text {true }}\right)=\sqrt{\left.\sum_{i}^{\text {Intranuke }} S_{i}^{2}\left(E_{\text {true }}\right)+\sum_{j}^{\text {hadronization }} S_{j}^{2}\left(E_{\text {true }}\right)\right)+S_{\text {Cali }}^{2}\left(E_{\text {true }}\right)} .
$$

Figure 5.15 shows the total combined systematics of $\mathrm{kNN}$ shower energy compared with the parameterization of its calorimetric shower energy.

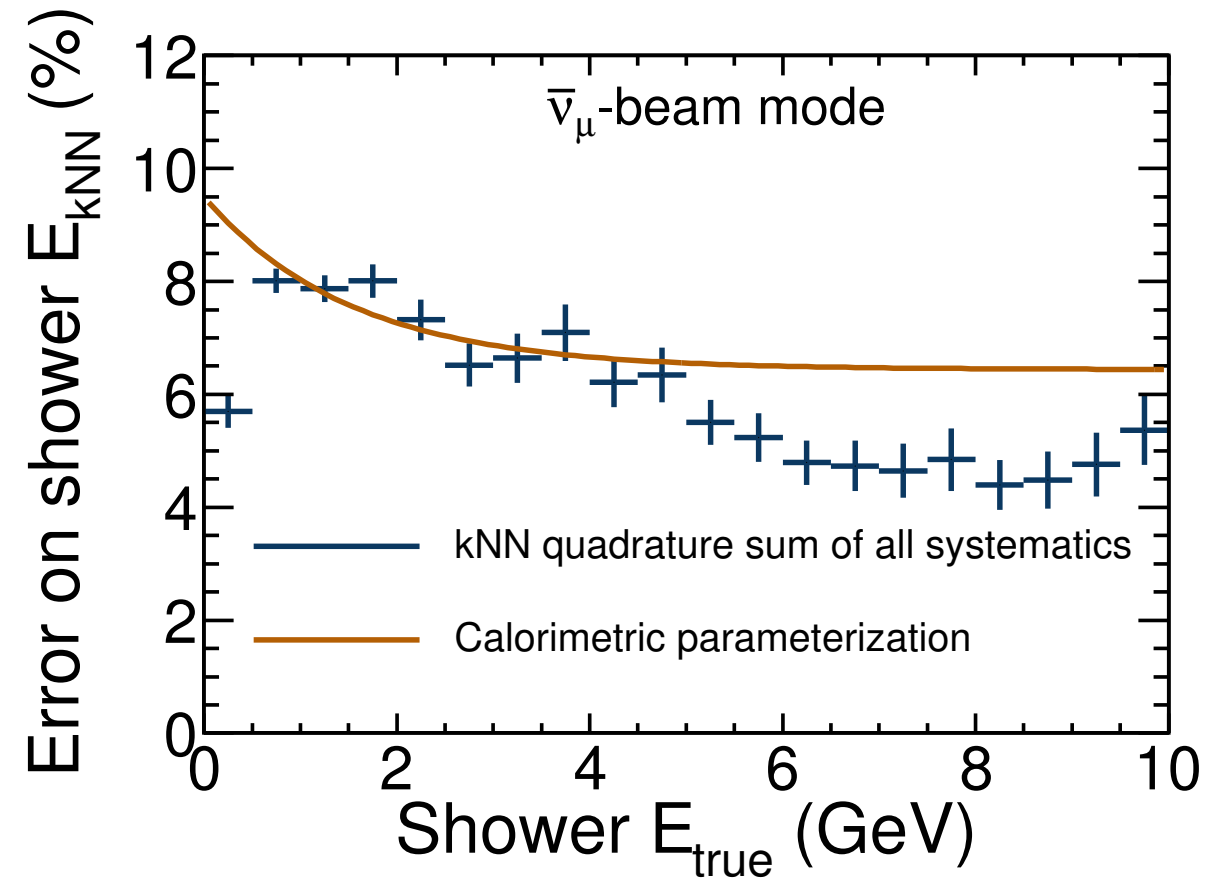

Figure 5.15: The total systematic error of kNN shower energy is shown in blue dots. The orange curve is the calorimetric parameterization which is fitted by the total systematic error of calorimetric shower energy. These are plotted with selected $\bar{\nu}_{\mu}$-CC events in the $\bar{\nu}_{\mu}$-beam mode. 
The calorimetric parameterization is derived from the total systematic error of the calorimetric shower energy. The identical approach is implemented for both kinds of the shower energy estimator. The overall systematic error of the $\mathrm{kNN}$ shower energy estimator is no worse than the systematic error of the calorimetric shower estimator.

\subsection{Summary of hadronic shower energy estimator}

This chapter introduced a new technique for estimating the hadronic shower energy. The power of this technique comes from exploiting multiple features of the dynamic and topological hit deposition of showers. These features, under supervision of the $\mathrm{kNN}$ algorithm generate the regression function to estimate shower energy for each event. Compared to the standard calorimetric estimator, the new technique yields better shower energy resolution and sensitivity to oscillation parameters. The sensitivity gained by kNN shower estimator is equivalent to a $10 \%$ statistical increase of the current data. A study of various systematic effects on the kNN shower energy estimator was performed. The overall systematic error of the kNN shower energy estimator is no worse than the systematic error of the calorimetric shower estimator. This validates that the kNN shower energy estimator is acceptable for the os-

cillation analysis, which will be discussed in Chapter 6, without introducing any further systematic uncertainty in comparison to the calorimetric shower energy. 


\section{Chapter 6}

\section{Analysis of accelerator charged-current muon antineutrino disappearance}

MINOS uses the same procedure for performing the measurements of accelerator charged-current (CC) $\nu_{\mu}$ and $\bar{\nu}_{\mu}$ disappearances. The analysis consists of three main components: (i) selecting data events for both detectors, (ii) predicting the Far Detector spectrum with the selected Near Detector data, and (iii) fitting certain oscillation parameters by comparing the selected Far Detector data to its prediction. In addition, data preselection is necessary to diminish the background, and systematics are incorporated in the fitting. The beam weight is also taken into consideration to tune the Monte Carlo simulation for better agreement with the selected Near Detector data. The main differences between the $\nu_{\mu}$ and $\bar{\nu}_{\mu}$ measurements are the event selections (a comparison showed in Section 4.4.1) and the beam matrices (introduced in Section 6.4) which are calculated independently for predicting the Far Detector $\nu_{\mu}$ and $\bar{\nu}_{\mu}$ spectra.

This chapter describes the accelerator $\bar{\nu}_{\mu}$-CC disappearance analysis pertained to data in the $\bar{\nu}_{\mu}$-beam mode. The analysis for $\bar{\nu}_{\mu}$-CC disappearance in the $\nu_{\mu}$-beam mode is performed similarly except for a difference in the event selections (described in Section 4.4). The analysis with the atmospheric 
$\bar{\nu}_{\mu}$-CC data, which is included in the measurement of the complete MINOS accelerator and atmospheric $\bar{\nu}_{\mu}$ data set, is described explicitly in [127].

\subsection{Accelerator charged-current $\bar{\nu}_{\mu}$ data preselection}

Many requirements are applied for preselecting accelerator $\bar{\nu}_{\mu}$-CC data events. First of all, data quality requires that only events collected in good operation periods of running are processed. The criteria for a good operation period relate to the proper horn current and the magnetic configurations in the MINOS and NuMI equipments. Figure 6.1 shows the periods of $\bar{\nu}_{\mu}$-CC data collected in the $\bar{\nu}_{\mu}$-beam mode and the stability of $\bar{\nu}_{\mu}$-CC energy spectra.

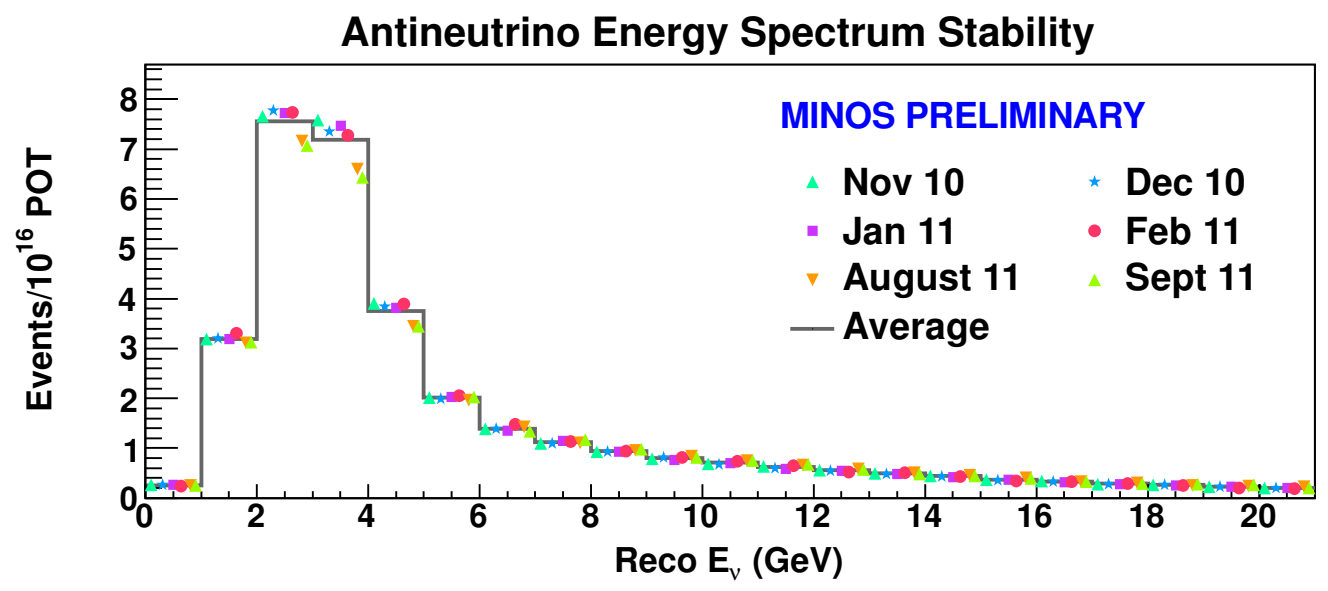

Figure 6.1: Number of $\bar{\nu}_{\mu}$-CC events in the $\bar{\nu}_{\mu}$-beam mode per $10^{16}$ protonson-target as a function of reconstructed neutrino energy in the Near Detector. The data are subdivided into calendar months. The last two points drop significantly due to the target decay [128]. 
To suppress background from atmospheric neutrinos and cosmic rays, preselected data events are required to be within a window of $[-2 \mu s, 12 \mu s]$ respectively to the nearest spill of the NuMI beam. Because of the more vertical direction of non-accelerator $\bar{\nu}_{\mu}$ events, the angle between the tracks

and the NuMI beam direction is used, $\cos \theta_{z}>0.6$, to preselect accelerator $\bar{\nu}_{\mu^{-}}$ CC data events at the Far Detector. Also, to make sure that the selected data events are completely reconstructed, their primary track vertices are required to be within a fiducial volume, which is defined independently for the Near and Far detectors as follows:

\section{Fiducial volume of the Near Detector}

The fiducial volume of the Near Detector is a cylindrical volume with radius of $0.8 \mathrm{~m}$ and around $3 \mathrm{~m}$ in length:

$$
\begin{aligned}
& \sqrt{\left(X_{\mathrm{vtx}}-X_{0}\right)^{2}+\left(Y_{\mathrm{vtx}}-Y_{0}\right)}<0.8 m, \\
& 0.81009 m<Z_{\mathrm{vtx}}-0.0392 m<4.07710 m,
\end{aligned}
$$

where $X_{0}=1.4828 \mathrm{~m}$ and $Y_{0}=0.2384 \mathrm{~m}$ are the coordinates of the coil hole center at the front face of Near Detector. Figure 6.2 shows the distribution of $X_{\mathrm{vtx}}$ and $Y_{\mathrm{vtx}}$ coordinates of selected $\bar{\nu}_{\mu}$-CC Near Detector data events in the $\bar{\nu}_{\mu}$-beam mode. Since the selected events should have their hadronic shower fully reconstructed, all recorded events with track vertices that occur out of the calorimeter containment, before the 14th plane or beyond the 68th plane, are removed from the Near Detector data, as seen in Figure 6.3. This definition of fiducial volume results in a corresponding mass of 23.7 tons. 

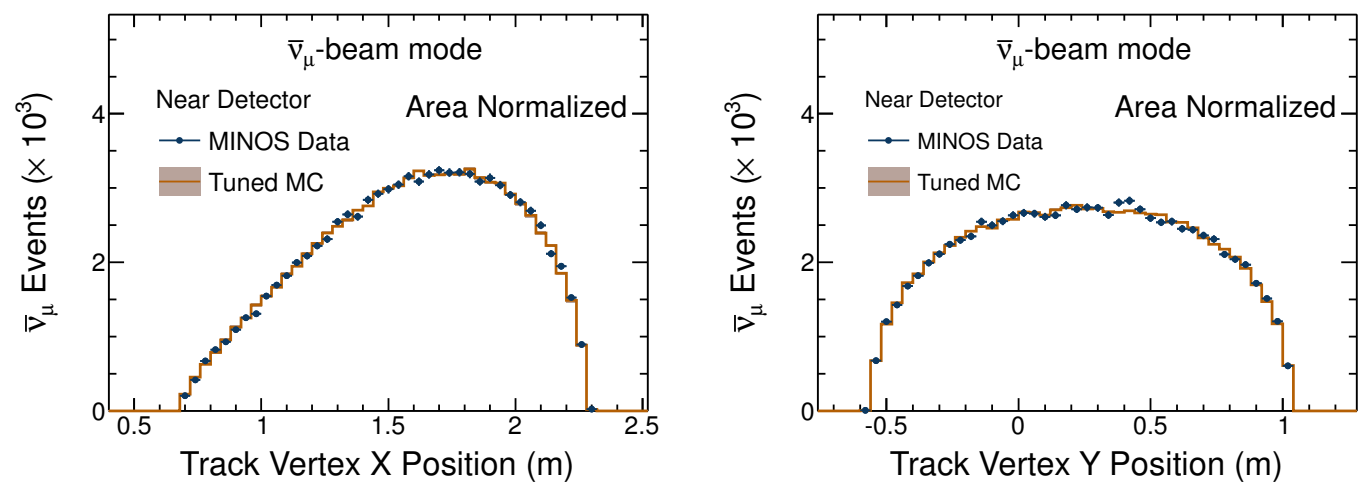

Figure 6.2: Distribution of $\mathrm{X}$ (left) and $\mathrm{Y}$ (right) coordinates of track vertices for selected $\bar{\nu}_{\mu}$-CC Near Detector data events in the $\bar{\nu}_{\mu}$-beam mode. The orange histogram represents the Monte Carlo prediction with systematic uncertainties and blue points represent data.

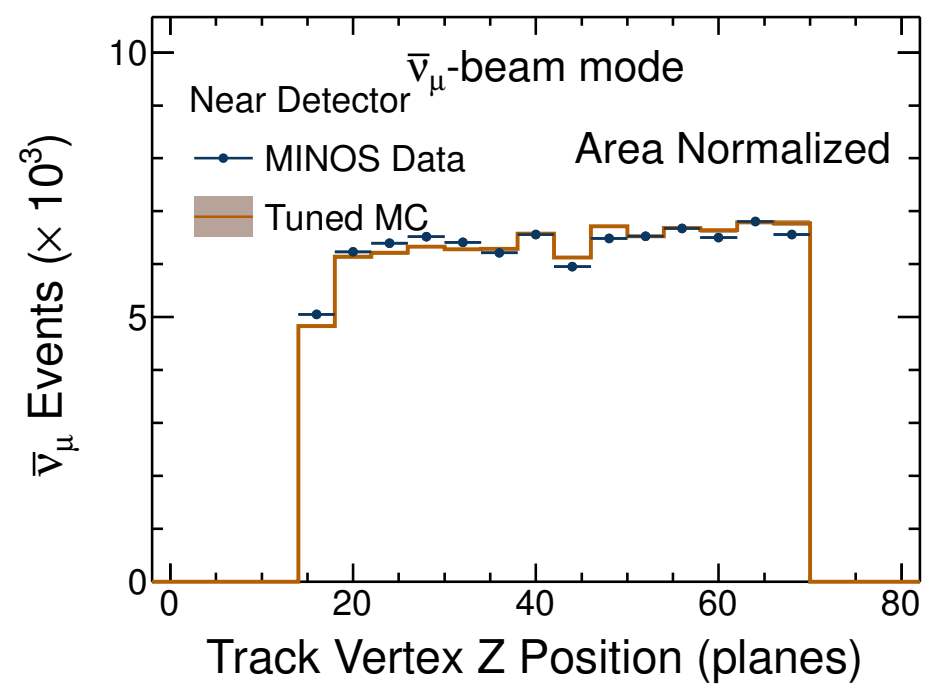

Figure 6.3: The Near Detector track vertex longitudinal position distribution in planes for selected $\bar{\nu}_{\mu}$-CC Near Detector data events in the $\bar{\nu}_{\mu}$-beam mode. The orange histogram represents the Monte Carlo prediction with systematic uncertainties and blue points represent data. 


\section{Fiducial volume of the Far Detector}

The fiducial volume of the Far Detector is the cylindrical volume with a radius of $r=3.7 \mathrm{~m}$ from the detector center and at least $50 \mathrm{~cm}$ away from the front and back planes of the two super-modules. Figure 6.4 shows the distribution of the track vertex and track end in $\mathrm{Y}$ vs. $\mathrm{X}$ coordinates for selected $\bar{\nu}_{\mu}$-CC Far Detector data events in the $\bar{\nu}_{\mu}$-beam mode. The effect of magnetic field, which focuses $\bar{\nu}_{\mu}$, can be conceived from this figure. This definition of fiducial volume results a corresponding mass of 4.17 kilotons (out of 5.4 kilotons in total) for the active region of the Far Detector.
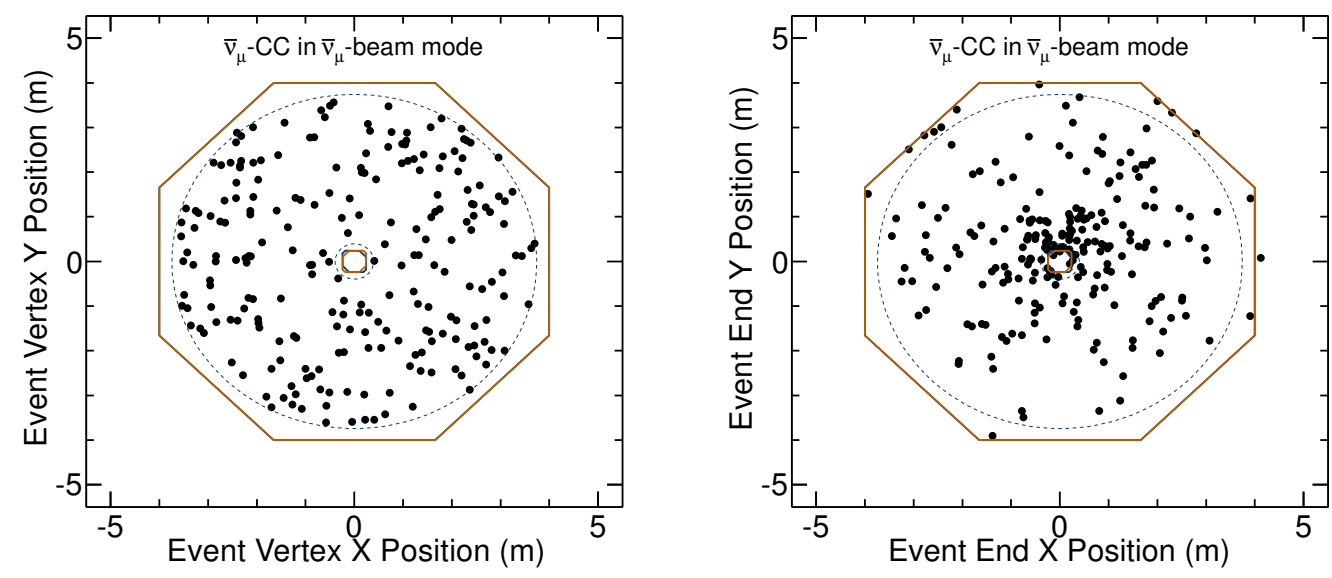

Figure 6.4: Distribution of track vertex (left) and track end (right) in Y vs. X coordinates for selected $\bar{\nu}_{\mu}$-CC events in $\bar{\nu}_{\mu}$-beam mode. 


\subsection{Accelerator charged-current $\bar{\nu}_{\mu}$ data selection}

The data event preselection is to filter only the good beam data set. After this step, a substantial background still remains and needs to be rejected for achieving a highly pure set of $\bar{\nu}_{\mu}$-CC data events. The accelerator $\bar{\nu}_{\mu}$-CC data events come from two data sets: (i) $\bar{\nu}_{\mu}$-CC data events in the $\bar{\nu}_{\mu}$-beam mode and (ii) $\bar{\nu}_{\mu}$-CC data events in the $\nu_{\mu}$-beam mode. In the standard approach, the candidate $\bar{\nu}_{\mu}$-CC data events in the first set is selected by two variable cut-based method. The first cut is on the $\mathrm{CC} / \mathrm{NC}$ separation parameter $(>0.3)$, as described in Section 4.4. This cut is to efficiently select the events with muon tracks without regard to their charge signs. The candidate $\bar{\nu}_{\mu}$-CC data events then are chosen based on the second cut on the charge sign measurement $(q / p>0)$, as discussed in Section 4.2. Figure 6.5a shows the agreement of $\mathrm{CC} / \mathrm{NC}$ separation parameter and charge-sign measurement between the selected $\bar{\nu}_{\mu}$-CC Near Detector data and Monte Carlo prediction in the $\bar{\nu}_{\mu}$-beam mode. 


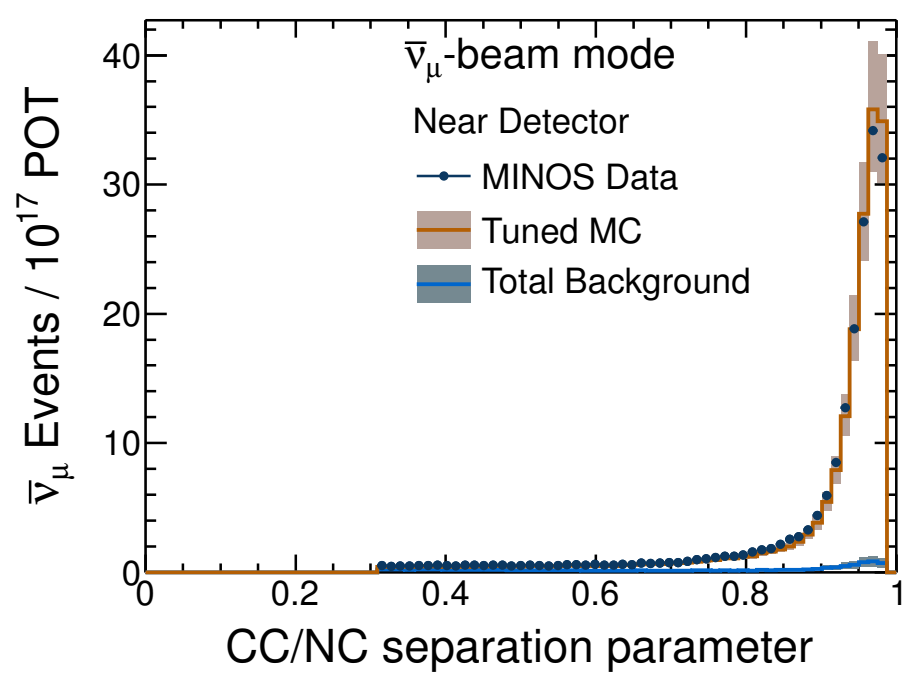

(a)

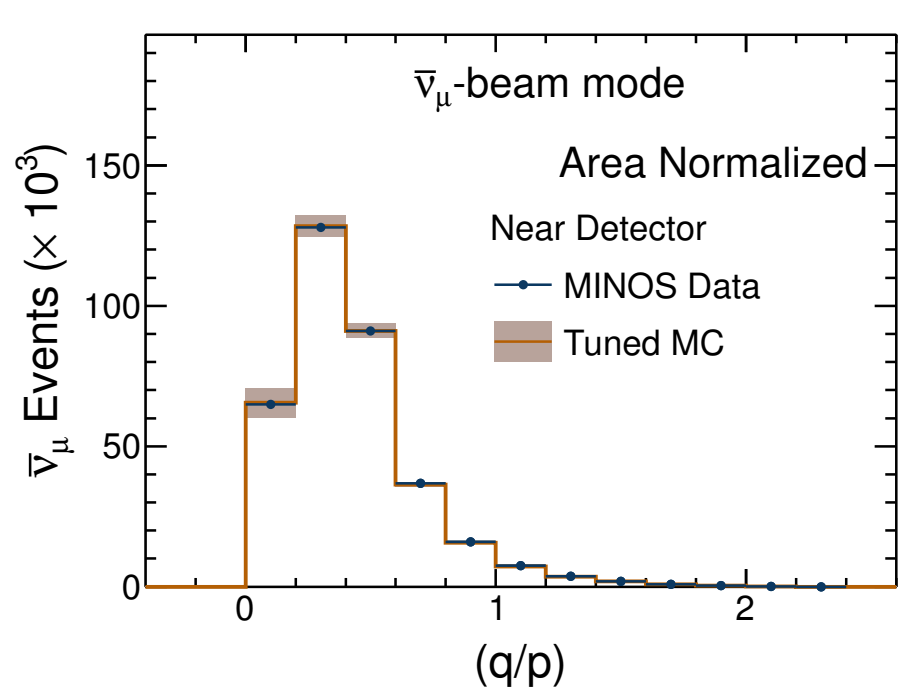

(b)

Figure 6.5: The $\mathrm{CC} / \mathrm{NC}$ separation parameter (left) the $(\mathrm{q} / \mathrm{p})$ distribution (right) of selected $\bar{\nu}_{\mu}$-CC Near Detector data events. The red histogram represents the Monte Carlo prediction with the systematic errors, the blue histogram represents the total (charged-current and neutral current) background. Black points represent data. 


\subsection{The $\bar{\nu}_{\mu}$-beam flux reweighting}

Figure 6.6 shows the comparatively notable disagreement of energy distribution between the Near Detector data and the default (untuned) Monte Carlo simulation.
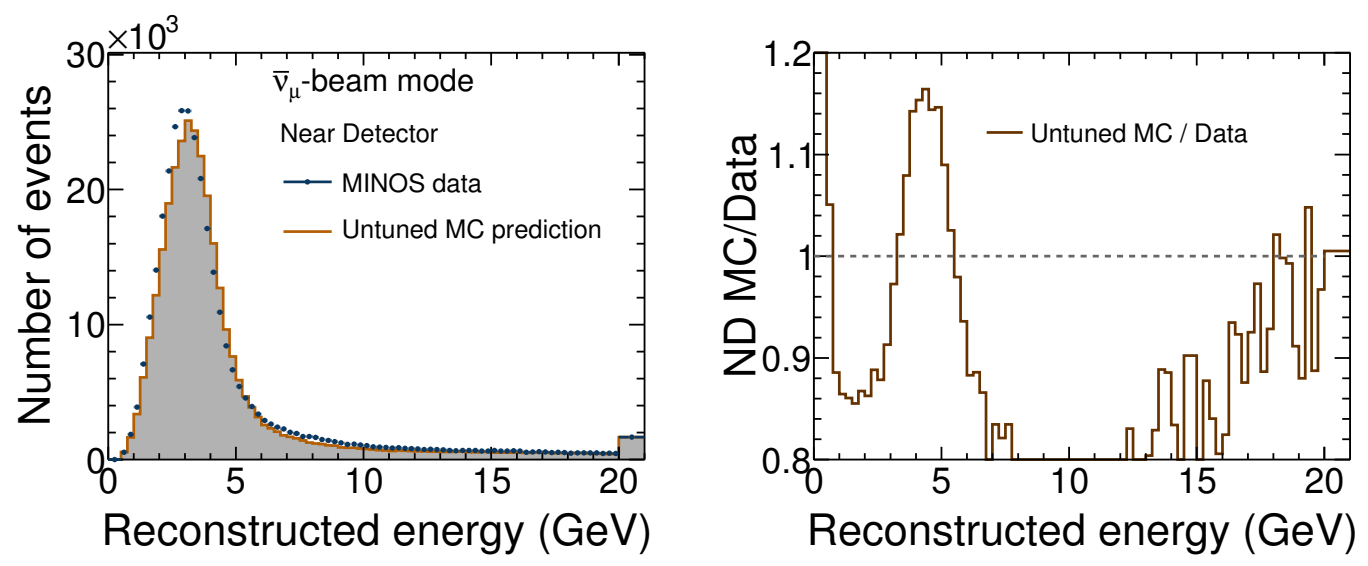

Figure 6.6: The discrepancy between selected $\bar{\nu}_{\mu}$-CC Near Detector data and default (untuned) Monte Carlo prediction. The energy spectrum is shown on the left and the ratio of Monte Carlo to data is shown on the right.

The lack of consistency is mainly due to a mismodelling of the neutrino beam flux, which is generated via a sequence of three steps: (i) simulating the creation of mesons when protons hit on the carbon target $p+C \rightarrow \pi^{ \pm} X$ or $K^{ \pm} X$, (ii) simulating the dynamics and interactions of these meson through the horns, decay pipe, and shielding, and (iii) calculating the weights of each individual mesons to decay into a neutrino of a given energy when seeing at the Near and Far detectors.

To enforce the agreement between the Monte Carlo simulation and Near 
Detector data, a reweighting procedure, named $\mathrm{SKZP}^{1}$, has been adopted [129]. The main idea is to parameterize the momentum distribution $\left(x_{F} \approx\right.$ $\left.\frac{p_{z}}{p_{\text {proton }}}, p_{T}\right)$ of hadrons produced in step (i) and vary their parameters to obtain consistency between the Monte Carlo simulation and Near Detector data. Also, to address the uncertainty of horn monitoring and the performance of the detectors, additional parameters such as horn current and position, absolute energy scale, antineutrino cross-section, etc., are included in the fitting. The flexibility of the NuMI beam, such as switching between the three neutrino beams (low, medium and high energy), adjusting the horn off or on, and varying the horn current, allows us to explore the relation between the neutrino energy and the hadron $\left(x_{F}, p_{T}\right)$ distributions in different regions of the parameter space. These relations are finally used to reweight the pion and kaon fluxes and thereby tune the energy distribution of neutrinos. Figure 6.8 shows the significant improvement of data and Monte Carlo agreement by applying the SKZP reweighting. The tuned Monte Carlo simulation shows better agreement with Near Detector data at the energy peak and increases the neutrino flux at high energy region.

The SKZP recalculates the neutrino energy distribution that would take an impact on the ratio of the Near to Far spectra, as illustrated in Figure 6.8. About 5\% discrepancy is observed. However this happens at about $4 \mathrm{GeV}$, which is away from the oscillation dip of around $1.5 \mathrm{GeV}$. Thus, the effect of flux reweighting, as shown in Appendix B, is fairly small.

\footnotetext{
${ }^{1}$ Abbreviated after Sacha Kopp, Zarko Pavlovic and Patricia Vahle
} 

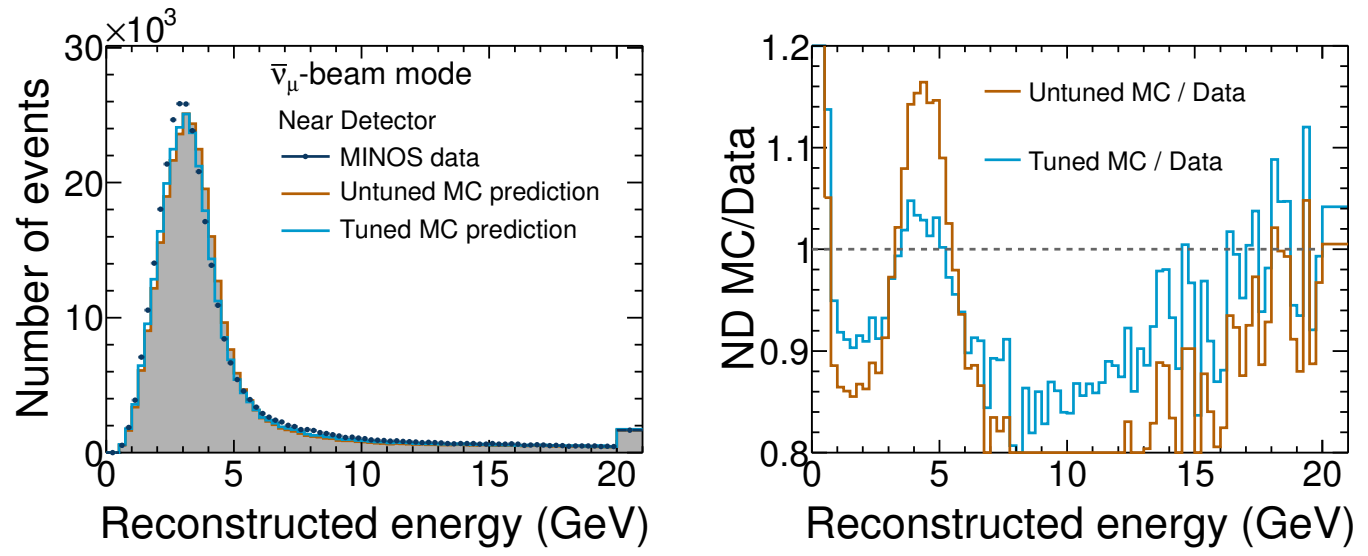

Figure 6.7: Comparison between the selected $\bar{\nu}_{\mu}$-CC Near Detector data and the Monte Carlo simulation before and after applying the SKZP reweighting. The spectra are shown on the left and the ratio of Monte Carlo to data is presented on the right.
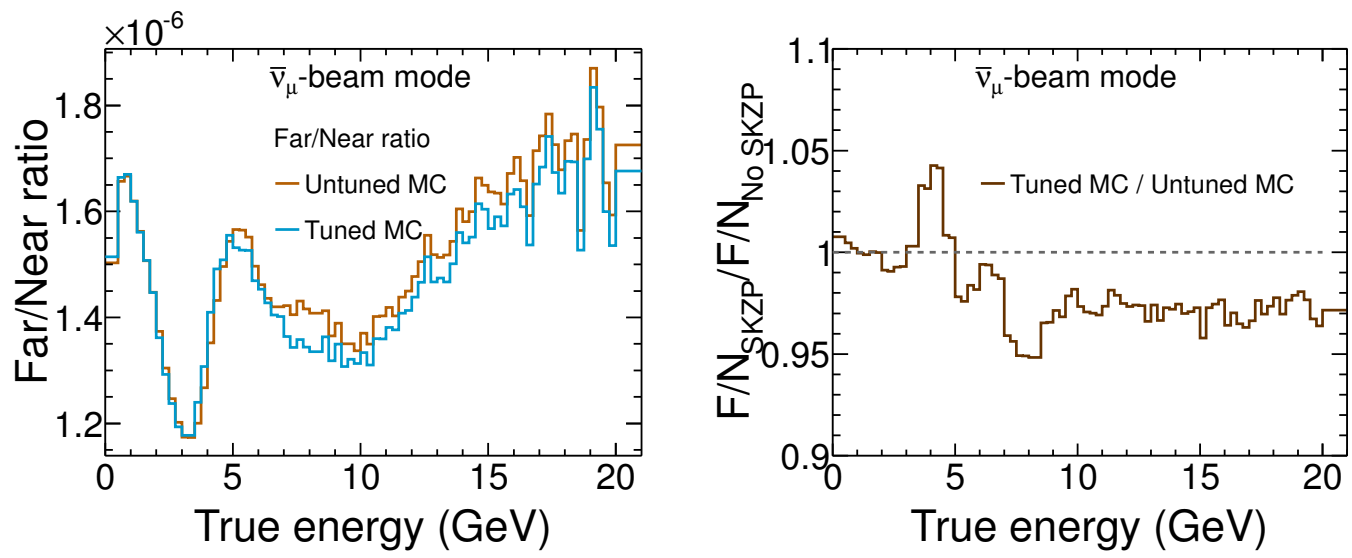

Figure 6.8: The discrepancy between selected $\bar{\nu}_{\mu}$-CC Near Detector data and Monte Carlo simulation and the effect of applying the SKZP reweighting. The spectrum is on the left and the ratio of Monte Carlo to data is on the right. 


\subsection{Far Detector prediction}

The two-detector design in the MINOS experiment permits one to directly predict the Far Detector spectrum with a substantial cancellation of systematic uncertainties. However, this cancellation only works perfectly if the neutrino energy spectra at the Near and Far detectors are identical. The fact is that the energy of the neutrino reaching the detectors depends on the angle of the neutrino momentum with respect to the $\pi^{ \pm}, K^{ \pm}$parent direction via the following formula:

$$
E_{\nu}=\left(1-\frac{m_{\mu^{2}}}{m_{\pi, K}^{2}}\right) \frac{E_{\pi, K}}{1+(\gamma \theta)^{2}}
$$

Figure 6.9 sketches the different angular acceptances to meson parents between the Near Detector and the Far Detector. This difference yields a shift on the energy spectra at the Far Detector compared with the Near Detector as illustrated in Figure 6.10.

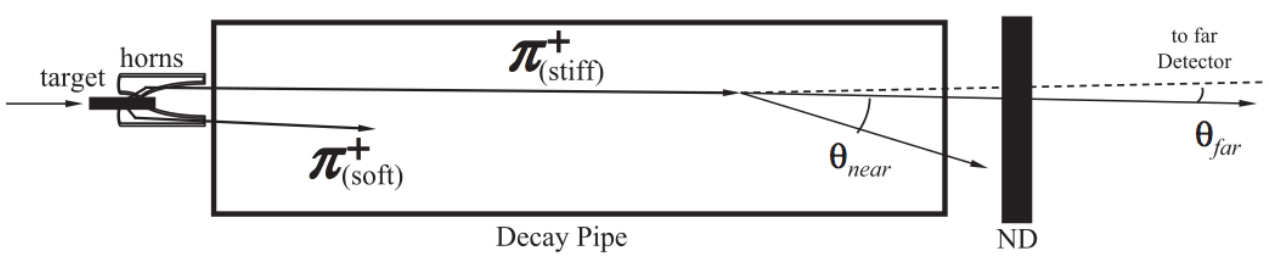

Figure 6.9: The different angular acceptance between the Near Detector and Far Detector from the view of pion decay points. Figure taken from [48]. 

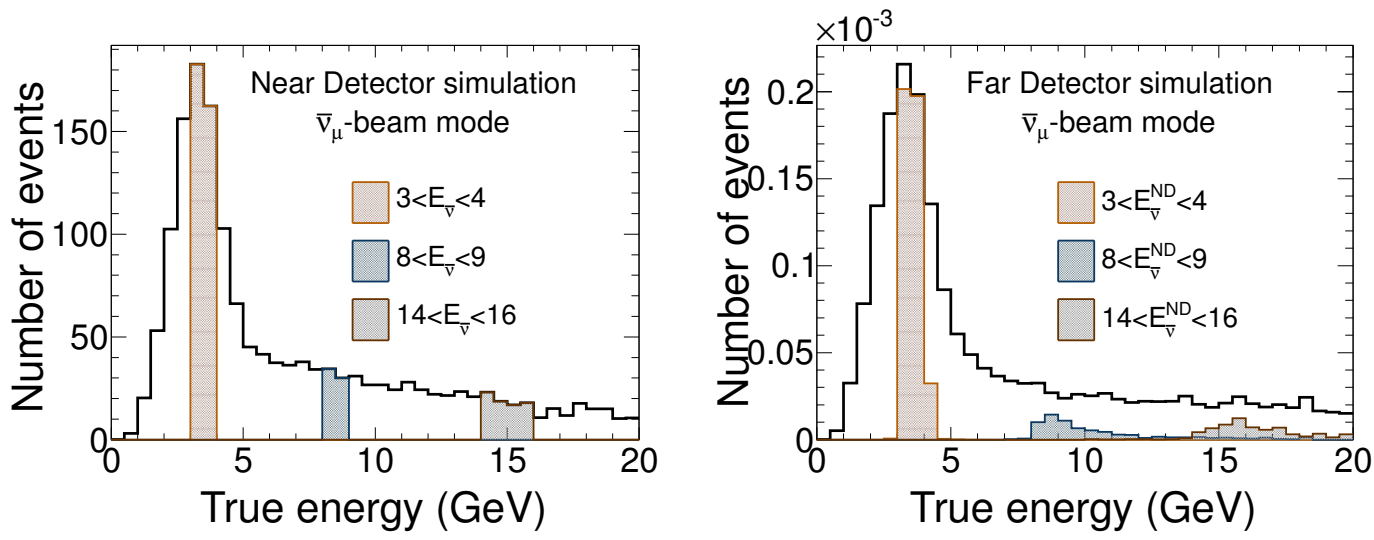

Figure 6.10: Simulated true $\bar{\nu}_{\mu}$-CC energy spectra in the Near Detector (left) and Far Detector (right). The meson parents, which are responsible for producing $\bar{\nu}_{\mu}$ in a given energy at the Near detector, produce a range of $\bar{\nu}_{\mu}$ energy distribution in the Far Detector.

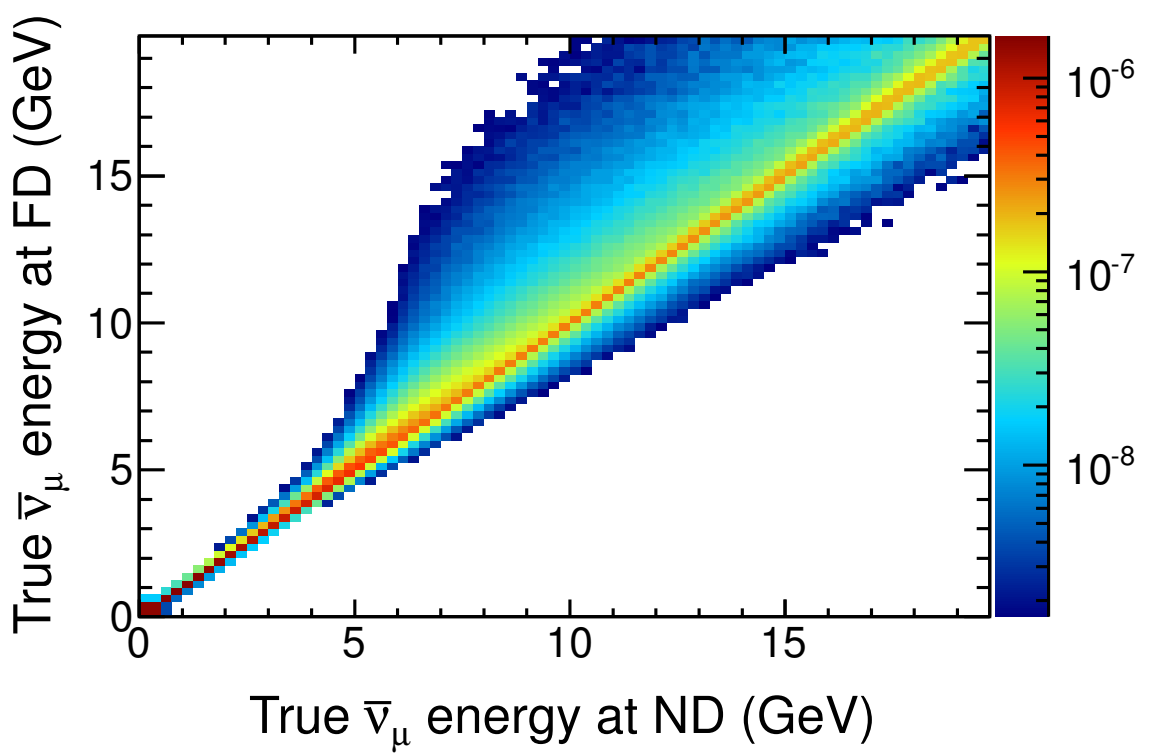

Figure 6.11: The transfer matrix used to convert $\bar{\nu}_{\mu}$ flux at the Near Detector into the Far Detector flux. 
The Far Detector flux is calculated from the observed Near Detector flux along with the beam Monte Carlo simulation. This can be achieved by applying a transfer (beam) matrix which essentially links the neutrino energy at the Near Detector to one at the Far Detector via their shared $\pi^{ \pm}, K^{ \pm}$parents. The detailed description of beam matrix can be found in [130]. Figure 6.11 shows the two-dimensional transfer matrix used to convert $\bar{\nu}_{\mu}$ flux at the Near Detector into the Far Detector flux. The nearly diagonal matrix shows the strong correlation between true $\bar{\nu}_{\mu}$ energy between two detectors. Because both detectors do not measure directly the neutrino flux but rather the event rate, additional calculations are needed to complete the extrapolation procedure: (i) converting the Near Detector data into the flux at Near Detector, and (ii) converting the Far Detector flux into the visible energy spectrum predicted at the Far Detector. Figure 6.12 shows the schematic extrapolation procedure to make the Far detector prediction from the Near Detector data.

\section{(i) Retrieving the Near Detector flux from the Near Detector data ${ }^{2}$ :}

First, the reconstructed energy spectrum at the Near Detector is corrected by multiplying with a purity correction to obtain the pure signal spectrum. This reconstructed spectrum is then converted to true energy spectrum by applying a reconstructed-to-true transferred matrix ${ }^{3}$. Next, the produced true energy spectrum is scaled by an efficiency correction to get a true energy spectrum of

\footnotetext{
${ }^{2}$ this assumes no oscillation occurred at the Near Detector

${ }^{3}$ two-dimensional histogram filled by the reconstructed and true energy value of selected signal events in the sample of the Near Detector Monte Carlo simulation
} 
all generated signal interactions in the fiducial volume at the Near Detector. Finally, the resultant true energy spectrum is converted to the flux at the Near Detector by scaling with the cross-sections and its fiducial volume.

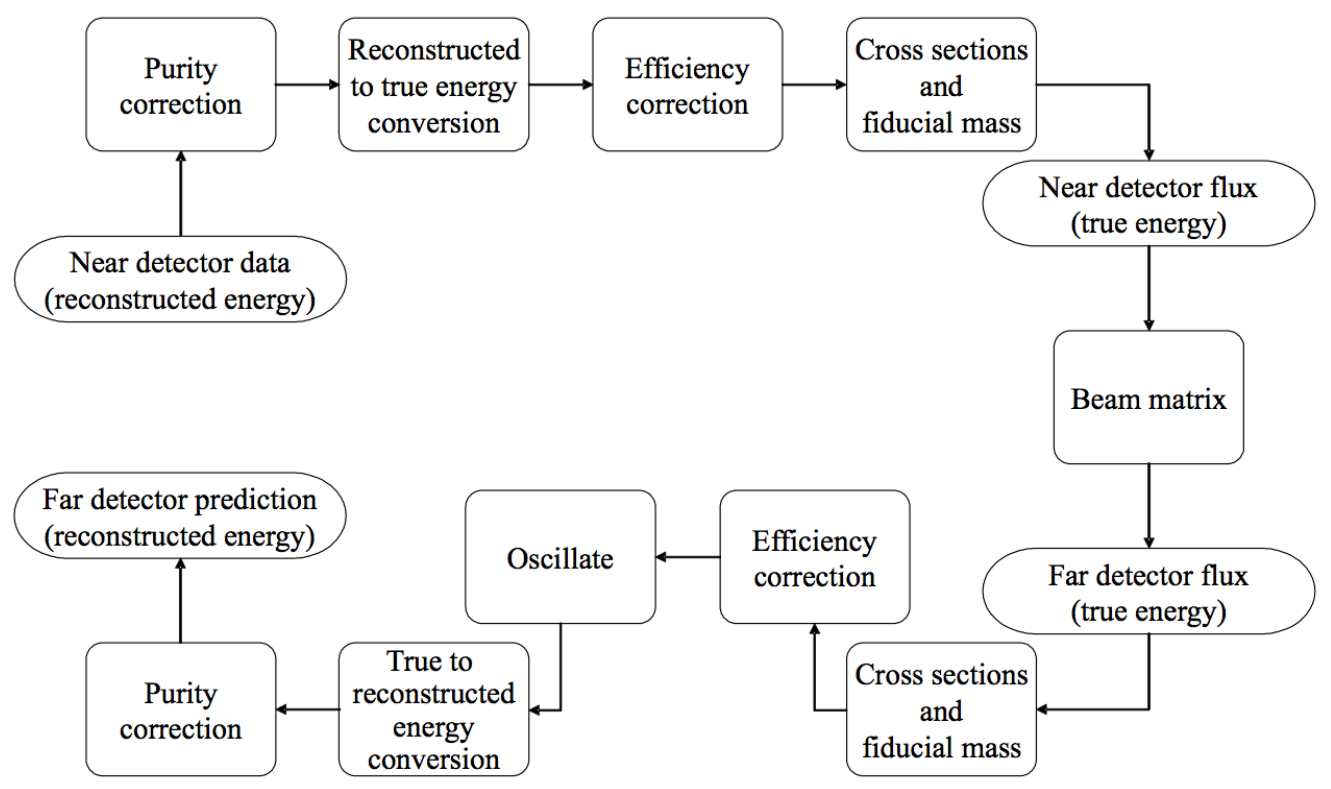

Figure 6.12: The scheme of beam extrapolation of the MINOS charged-current $\nu_{\mu}$ disappearance analysis. Figure taken from [130].

(ii) Predicting the reconstructed Far Detector spectrum from the

Far Detector flux: This basically is in the reverse order of retrieving the above calculation. Firstly, the Far Detector flux is scaled by the cross-section, the fiducial mass at the Far Detector, and the given exposure of the Far Detector data. The efficiency correction then is applied to get the pure signal distribution as a function of the true energy which has possibly been seen by the Far Detector. Next, this is converted into the spectrum of reconstructed 
energy by multiplying it with a true-to-reconstructed transferred matrix ${ }^{4}$. The oscillation probability can be applied before this step to make the Far Detector prediction with a given set of oscillation parameters. Finally, the purity correction is implemented to get the ultimate prediction which is used to compare with the Far Detector data.

The extrapolation procedures for the charged-current $\nu_{\mu}$ and $\bar{\nu}_{\mu}$ disappearance analyses are slightly different. For the former analysis, only the $\nu_{\mu}$ spectrum is extrapolated; while the $\bar{\nu}_{\mu}$ and $\nu_{\mu}$ spectra are extrapolated individually for the latter analysis. The motivation behind this is the wrong-sign background for the latter analysis is fairly large in comparison to its for the former analysis. Also, the separation of $\bar{\nu}_{\mu}$ and $\nu_{\mu}$ spectra in extrapolation allows us to manifest the different sets of oscillation parameters for neutrino and antineutrino oscillations, giving a test of CPT invariance in the lepton sector. Figure 6.13 shows the schematics for extrapolating $\bar{\nu}_{\mu}$ and $\nu_{\mu}$ spectra individually.

To validate the framework of beam extrapolation, Near Detector Monte Carlo fake data, which are used to calculate reconstructed-to-true matrix at the Near Detector, are extrapolated. The prediction then is compared with Far Detector Monte Carlo fake data, which are used to calculate true-to-reconstructed matrix at the Far Detector. The prediction is expected to agree very well with the Far Detector fake data. Figure 6.14 shows that the agreement between

\footnotetext{
${ }^{4}$ similar to the reconstructed-to-true transferred matrix at the Near Detector but switching between two axes and using the Far Detector Monte Carlo sample
} 


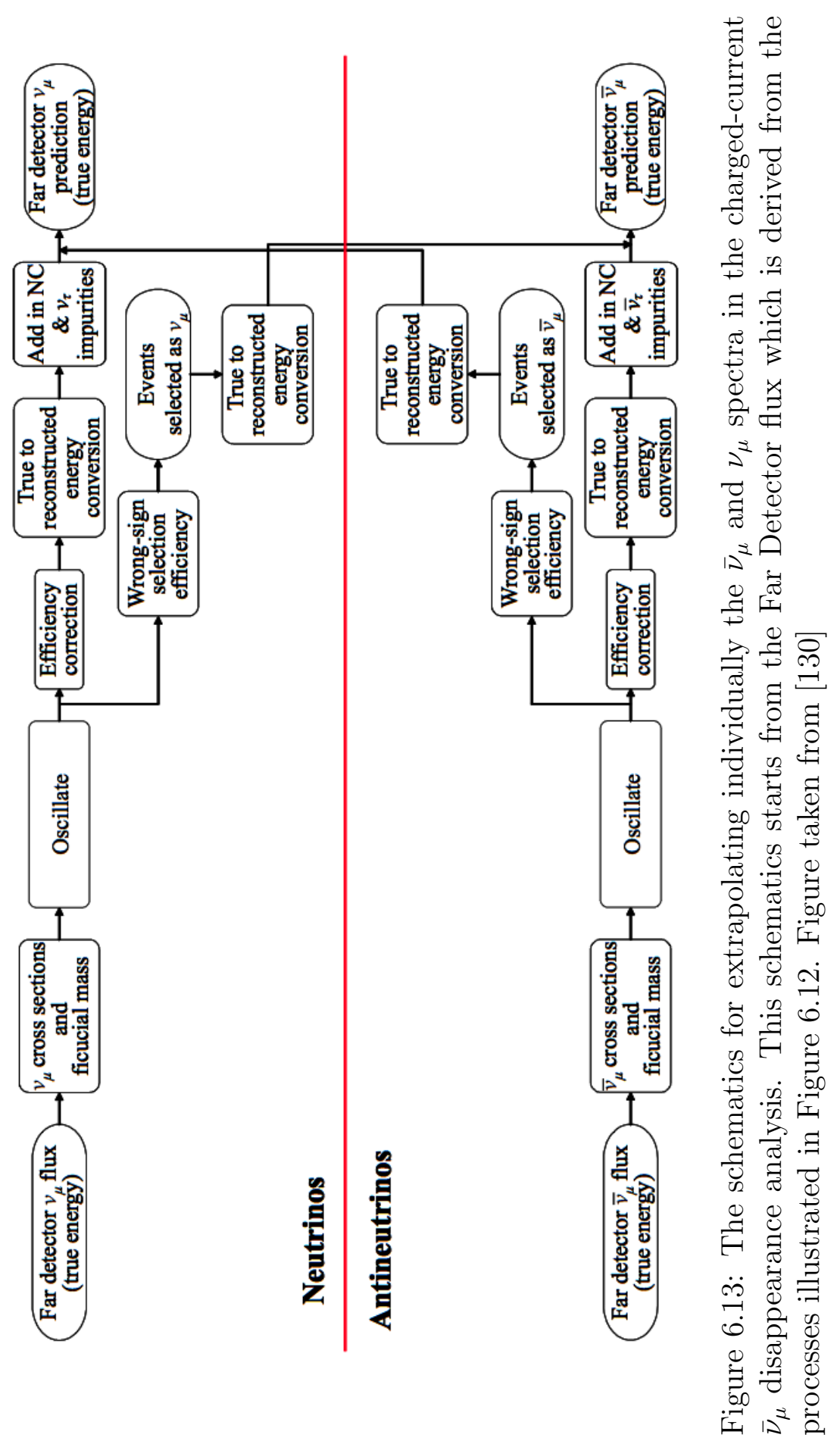


the Far Detector fake data and its prediction is within 1\%. The fluctuation is unavoidable due to the statistical independence among samples used for making matrices. However this fluctuation is still good enough for the purpose of analysis since this is much smaller than the statistical uncertainty of the Far Detector data.
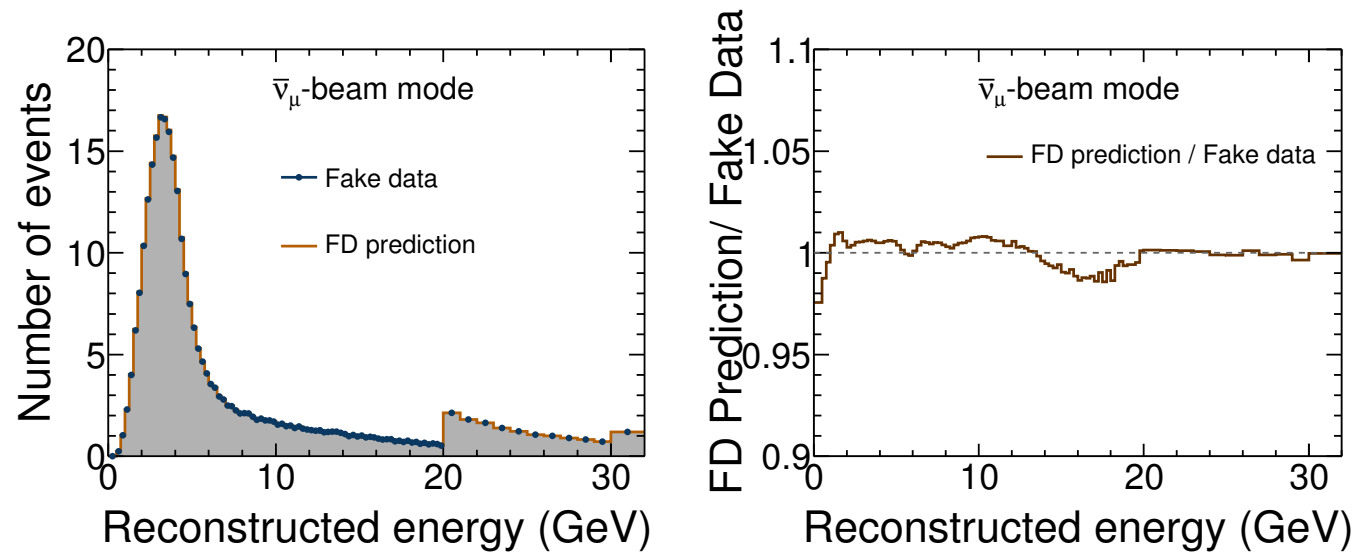

Figure 6.14: The comparison between the prediction and the FD fake data spectra is on the left and the ratio of the prediction to the FD fake data is on the right.

\subsection{Systematic error evaluation}

The main sources of uncertainties for the $\bar{\nu}_{\mu}$-CC disappearance analysis arise from the limited precision of modeling neutrino production, neutrino interactions, measurement of detector properties and the functional adjoint of the two detectors. These uncertainties take effect on an estimation of oscillation parameters by either distorting the reconstructed energy spectra or estimating incorrectly the number of signal or background. While the dis- 
torted reconstructed energy spectra would result in error of estimating the dip

of oscillation, i.e., the value of $\left|\Delta \bar{m}^{2}\right|$, the bias in counting of signals and backgrounds would mainly shift the value of $\sin ^{2} 2 \bar{\theta}$. The following ten systematics are broadly categorized into four categories (i) beam-related uncertainties, (ii) background-related uncertainties, (iii) energy-related uncertainties, and (iv) normalization-related uncertainties.

\section{Beam-related uncertainties}

- Flux modeling: The uncertainties in the NuMI beam simulation primarily relate to the production rate of hadrons off the NuMI target. The prediction of neutrino flux from the NuMI beam fluctuates largely between 20-30\% from comparison of various theoretical models [131]. Other minor uncertainties arise from the models of beam optics, target position, and horn focusing [132]. Beam reweighting uses a set of parameters to tune the Monte Carlo for better agreement with the Near Detector data. These parameters, obtained by fitting data to Monte Carlo, are given $\pm 1 \sigma$ errors. However this uncertainty is fully correlated between two detectors and thus is nearly canceled out when extrapolating from the Near Detector to the Far Detector.

- Acceptance: This uncertainty is included to cover the difference of acceptances to $\mu^{-}$and $\mu^{+}$tracks in the Near Detector. To evaluate this uncertainty, special data were taken with an operating configuration in which the coil current is reversed to de-focused $\mu^{+}$tracks produced by 
$\bar{\nu}_{\mu}$-CC interactions. The ratio of the prediction from this special Near Detector data to the prediction from a normal run in which $\mu^{+}$tracks from $\bar{\nu}_{\mu}$-CC interactions are focused, is calculated and incorporated as uncertainty of the analysis.

- Cross-section: The total $\bar{\nu}_{\mu}$-CC cross-section is mainly comprised of three components: quasi-elastic scattering, resonance production and deep inelastic scattering. Each of these components are modeled with a number of uncertainties. The uncertainty-evaluated procedure is to compare the cross-section modes with varied parameters and the nominal ones.

\section{Background-related uncertainties}

- Neutral-current background: The main background of charged-current analysis is the neutral-current events. The uncertainty of this background is estimated by using the fake neutral-current events which are formed by removing the muon-liked track from selected charged-current events in both the data and Monte Carlo. A scale factor of $50 \%$ is placed for this systematic error for both detectors [130].

- Wrong-signed background: The $\mathrm{CC} / \mathrm{NC}$ separation is used to select the charged-current events (both $\bar{\nu}_{\mu}$ and $\nu_{\mu}$ ). The systematic error for this selection is smaller than $0.5 \%$ [109]. To select specifically $\bar{\nu}_{\mu}$-CC events, positive charge-sign value of tracks are required. Since the charge sign measurement is based on the track curvature in the magnetic field, 
it might be biased by the multiple scattering of muons, alignment of detector planes and knowledge of magnetic field in detector. To account for these, a $50 \%$ scale factor is applied separately for both detectors [130].

\section{Energy-related uncertainties}

The reconstructed $\bar{\nu}_{\mu}$-CC energy distributions are obtained from the sum of the $\mu^{+}$track energy and hadronic shower energy. Any bias in energy estimation would result in error for estimating the dip of oscillation, thus this shifts the value of $\left|\Delta \bar{m}^{2}\right|$.

- Shower energy: The uncertainties for estimating shower energy, disused in Section 5.5, are mostly dominated by the absolute energy calibration factor of $5.7 \%$, relative energy calibration factor of $2.4 \%(2.3 \%)$ at the Far Detector (the Near Detector), and uncertainty from the hadronic modeling. These uncertainties are uncorrelated, and therefore added in quadrature to give a parameterized total uncertainty by [120]:

$$
\sigma_{\text {shower }}=6.7 \%+3.5 \% * e^{-E_{\text {shw }} / 1.44 G e V}
$$

- Track energy: Two independent methods are implemented for estimating the track energy of events: (i) the muon range, and (ii) the curvature of muon tracks. To take the advantage of these two methods, the stopped muons are measured by range while the exiting muons are estimated by their curvature. A $2 \%$ uncertainty on all range of track energy, which was evaluated by comparing the Monte Carlo simulation 
and Calibration Detector responses, is taken [48]. This accounts for the uncertainty in the knowledge of steel density and muon energy loss in iron. An additional 1\% error from the curvature estimation is included for the uncertainty of the magnetic fields in detectors.

\section{Normalization-related uncertainties}

To maximize the statistics, the Far Detector data is used as much as possible. For the Far Detector prediction, the Near Detector data is required to normalize to the proton-on-target (POT) of the Far Detector data. The Nearto-Far POT normalization suffers some source of systematics errors. They mainly consist of a $1 \%$ of exposure time of the Far Detector, $2 \%$ of fiducial mass of the Far Detector, and 3\% of track reconstruction efficiency. A quadrature sum yields a total of $4 \%$ normalization uncertainty.

Figure 6.15 shows the total systematic error as the function of reconstructed energy at the Near Detector. The total uncertainty of the Near Detector energy spectrum is around $20 \%$ at the $2-6 \mathrm{GeV}$ region. However, the uncertainty of the Far Detector should be much smaller than this value because of the substantial cancellation of two-detector extrapolation. Figure 6.16 shows the total systematic error on the Far Detector prediction. The total uncertainty is around $8 \%$ at the $2-6 \mathrm{GeV}$ region. This estimated uncertainty is not used in the oscillation parameter fitting but is incorporated for producing the error band of the Monte Carlo prediction with the best-fit point in comparison to the Far Detector data. 


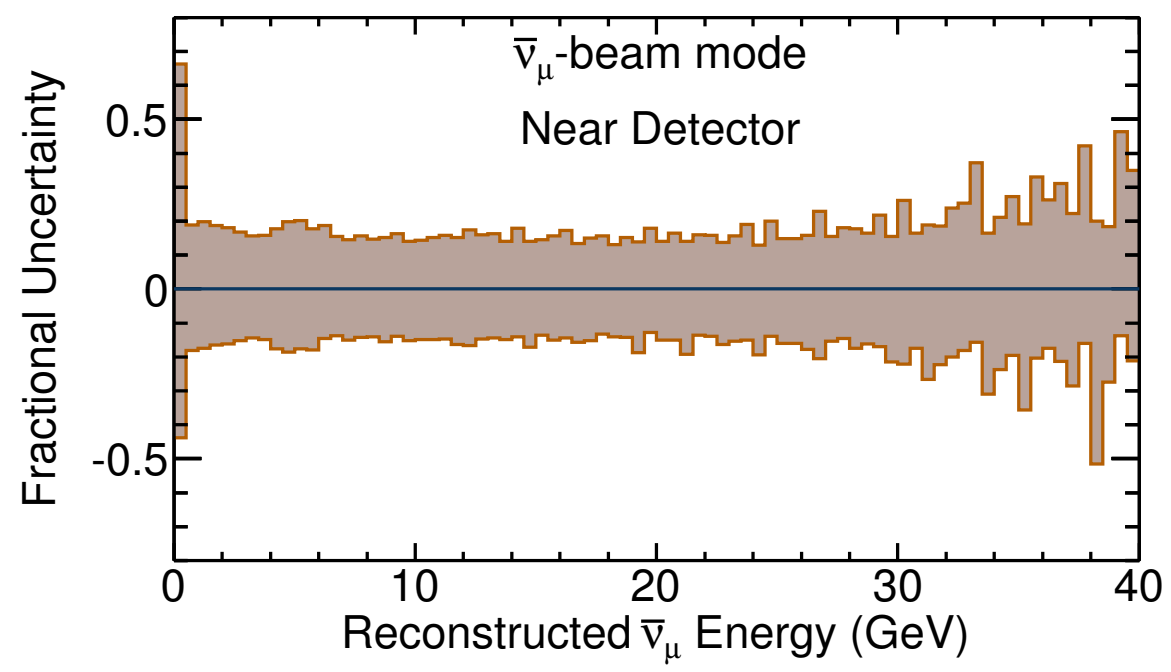

Figure 6.15: The Near Detector systematic error band as a function of reconstructed $\bar{\nu}_{\mu}$ energy with all systematic shifts included.

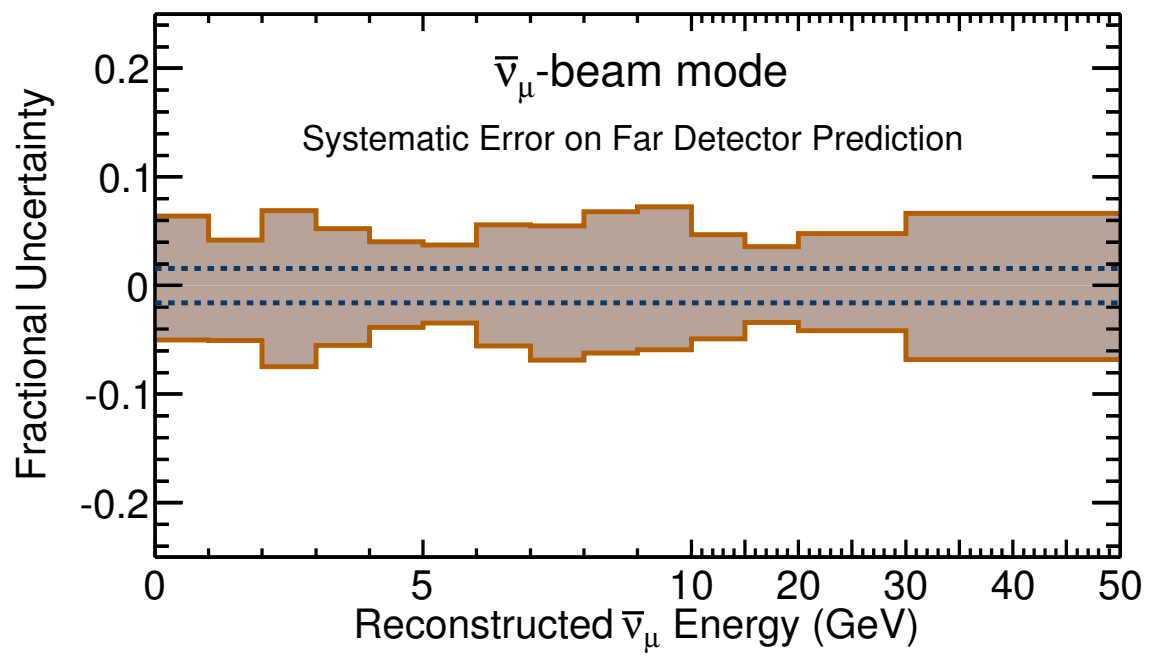

Figure 6.16: Total systematic error band on the Far Detector prediction. The band is obtained by adding the effect of each individual systematic shift on the FD predicted energy spectrum in quadrature. 


\subsection{Oscillation parameter fitting}

As discussed in Section 6.4, the Far Detector spectrum can be predicted from the Near Detector data with given oscillation parameters. To search the best-fit values of oscillation parameters for describing the $\bar{\nu}_{\mu}$-CC disappearance at the Far Detector, a grid of the considered oscillation parameters is created and the Far Detector predicted spectrum is generated for each grid point. A maximum log-likelihood approach is performed on the set of Far Detector predicted spectra to find the best match with the Far Detector data. The number of floated oscillation parameters in the grid depends on the applied oscillation model and physics goal. In the two-flavor model of $\bar{\nu}_{\mu}$-CC disappearance ${ }^{5}$, the $\bar{\nu}_{\mu}$ survival probability is effectively calculated by:

$$
P\left(\bar{\nu}_{\mu} \rightarrow \bar{\nu}_{\mu}\right)=1-\sin ^{2} 2 \bar{\theta} \sin ^{2}\left(1.267 \frac{\Delta \bar{m}^{2} L[k m]}{E[G e V]}\right)
$$

and $\Delta \bar{m}^{2}$ and $\sin ^{2} 2 \bar{\theta}$ are varied in the grid. The best-fit values of parameters are obtained by maximizing the following negative log-likelihood function:

$$
\chi^{2}\left(\left|\Delta \bar{m}^{2}\right|, \sin ^{2} 2 \bar{\theta}\right)=-2\left(\sum_{i=1}^{N_{\text {bin }}}\left(N_{i}^{\text {exp }}-N_{i}^{\text {obs }}\right)+N_{i}^{\text {obs }} \times \ln \frac{N_{i}^{\text {obs }}}{N_{i}^{\text {exp }}}\right)
$$

where $N_{\text {bin }}$ is the binning number of reconstructed energy spectra, $N_{i}^{\exp }$ and $N_{i}^{\text {obs }}$ are respectively the number of events in $i^{\text {th }}$ of reconstructed energy spectra of the Far Detector prediction and Far Detector data. The main systematic

\footnotetext{
${ }^{5}$ MINOS has recently fitted data using the three-flavor neutrino model. However this has a tiny impact on the antineutrino oscillation parameters since the matter effect is ignorable with a MINOS baseline/ $\bar{\nu}_{\mu}$ energy of $735 \mathrm{~km} / 3 \mathrm{GeV}$.
} 
errors are incorporated as nuisance parameters into the $\chi^{2}$ function by:

$$
\chi_{\text {systematics }}^{2}=-\sum_{j} \frac{\alpha_{j}^{2}}{\sigma_{\alpha j}^{2}}
$$

where $\alpha_{j}$ is the shift value of $j^{\text {th }}$ systematics from its nominal value, and $\sigma_{\alpha j}$ is the estimated uncertainty of $j^{\text {th }}$ systematic parameter. The minimum value of $\chi^{2}$, determined by performing the MINUIT package [133], corresponds to the best-fit values of oscillation parameters. The one-dimensional confidence level (C.L.) for the oscillation parameters are determined from the $\chi^{2}$ deviation, $\Delta \chi^{2}$, from the minimum $\chi^{2}$. For the Gaussian approximation, three basic following conversion are applied: $68 \%$ C.L at $\Delta \chi^{2}=1,90 \%$ C.L at $\Delta \chi^{2}=2.71$ and $99 \%$ C.L at $\Delta \chi^{2}=6.63$.

Normally, the whole spectrum of the Far Detector data is used to fit at the same time. However, it was found that by splitting the data set into different regions of fractional energy resolution, the sensitivity to oscillation parameters can be substantially improved [58]. Appendix C describes this technique in more detail and shows the sensitivity gain by adopting this technique for the $\bar{\nu}_{\mu}$-CC disappearance analysis. Unfortunately, due to time constraints, this technique is not incorporated in the final result measured in this thesis. The author of this thesis has adopted this technique for the $\nu_{\mu}$-CC disappearance analysis in the MINOS+ experiment. 


\subsection{Summary of the accelerator charged-current $\bar{\nu}_{\mu}$ dis- appearance analysis}

This chapter introduced the chain of the accelerator $\bar{\nu}_{\mu}$-CC disappearance analysis. The events with good beam quality and in the fiducial volume were preselected in both MINOS detectors. The PID then was implemented to efficiently purify the accelerator $\bar{\nu}_{\mu}$-CC data set. After that, the selected $\bar{\nu}_{\mu}$-CC events in the Near Detector were used for reweighting the $\bar{\nu}_{\mu}$-enhance beam flux. This step tuned the Monte Carlo simulation for better agreement between the Near Detector data. The tuned Monte Carlo simulation at the Near Detector was extrapolated by a transfer matrix to predict the Far Detector spectrum. The evaluation of systematics on the Far Detector prediction was followed. Finally, a maximum log-likelihood approach was introduced for finding the best-fit values of oscillation parameters which described best the $\bar{\nu}_{\mu}$-CC disappearance at the Far Detector. 


\section{Chapter 7}

\section{Results of the MINOS $\bar{\nu}_{\mu}$ charged-current disappearance analyses}

This chapter presents the measurements of antineutrino oscillation parameters from charged-current (CC) $\bar{\nu}_{\mu}$ disappearance at the MINOS Far De-

tector. First, three different data sets of $\bar{\nu}_{\mu}$-CC events are used for these measurements and the physics goals of this analysis are introduced. Then, the antineutrino oscillation parameters, measured by fitting the Far Detector data events from different data sets, are reported. Finally, an examination of CPT symmetry invariance in the lepton sector, by comparing the antineutrino and neutrino oscillation parameters, is performed.

\subsection{The complete MINOS $\bar{\nu}_{\mu}$ data set}

Three different data sets of $\bar{\nu}_{\mu}$-CC events are used for measurements in this dissertation: (i) accelerator $\bar{\nu}_{\mu}$-beam events, (ii) accelerator $\nu_{\mu}$-beam events, and (iii) atmospheric antineutrino events. The data exposure and previous results with these data sets are briefly described as follows: 


\section{Accelerator $\bar{\nu}_{\mu}$-CC events in the $\bar{\nu}_{\mu}$-beam mode}

This beam was specially operated to enhance the statistics of $\bar{\nu}_{\mu}$-CC events for measuring the antineutrino oscillation parameters. The current in the NuMI focusing horns was reversed in order to select $\pi^{+} / K^{+}$and the magnetic fields in MINOS detectors were also simultaneously reversed to focus the induced $\mu^{+}$from $\bar{\nu}_{\mu^{-}} \mathrm{CC}$ interactions. This beam consists of $39.9 \%$ of $\bar{\nu}_{\mu^{-}}$ $\mathrm{CC}, 58.1 \%$ of $\nu_{\mu}$-CC and $2.0 \%$ of $\nu_{e}$ and $\bar{\nu}_{e}-\mathrm{CC}$. MINOS reported the first direct observation of $\bar{\nu}_{\mu}$ disappearance [2] with an exposure of $1.71 \times 10^{20}$ protons on target (POT). By assuming that $\bar{\nu}_{\mu}$-CC and $\nu_{\mu}$-CC disappearance is driven by the same oscillation parameters, the independent $\bar{\nu}_{\mu}$ and $\nu_{\mu}$ measurements was found to be consistent at the $2 \%$ confidence level. This thesis updates this analysis with a total data exposure of $3.36 \times 10^{20} \mathrm{POT}$ with the $\bar{\nu}_{\mu}$-beam mode.

\section{Accelerator $\bar{\nu}_{\mu}$-CC events in the $\nu_{\mu}$-beam mode}

The MINOS experiment mainly collected data from this beam configuration with a total $10.7 \times 10^{20} \mathrm{POT}$ exposure. The portion of $\bar{\nu}_{\mu}$ interactions in this beam is about $7.1 \%$. A measurement of $\bar{\nu}_{\mu}$-CC disappearance in this beam configuration was performed in 2011 [114]. Compared to the $7.1 \times 10^{20}$ POT exposures used in previous measurements, the statistics of this sample has been increased up to $10.5 \times 10^{20}$ POT exposure ${ }^{1}$ in this thesis.

\footnotetext{
${ }^{1} \mathrm{~A}$ small portion of this data set $\left(0.2 \times 10^{20} \mathrm{POT}\right.$ exposure $)$ was collected with a highenergy beam and is not used in this analysis.
} 


\section{Atmospheric $\bar{\nu}_{\mu}$-CC events}

The MINOS Far Detector collected 37.9 ktons-years of atmospheric neutrinos and antineutrinos from August 2003 to March 2011. The magnetic field of the Far Detector allows one to select $\bar{\nu}_{\mu}$-CC events on an event-by-event basis. The measurement of the atmospheric $\bar{\nu}_{\mu}$-CC disappearance in the Far Detector [127] was performed and shown to be consistent with atmospheric mass-squared splittings for neutrinos and antineutrinos, $\left|\Delta m^{2}\right|-\left|\Delta \bar{m}^{2}\right|=$

$0.6_{-0.8}^{+2.4} \times 10^{-3} \mathrm{eV}^{2}$. In the content of this thesis, the atmospheric $\bar{\nu}_{\mu}$ CC data set remains unchanged.

In the next sections, the result of $\bar{\nu}_{\mu}$-CC disappearance from the $\bar{\nu}_{\mu^{-}}$ beam mode is described first since this mainly constrains the antineutrino oscillation parameters. The $\bar{\nu}_{\mu}$-CC data in the $\nu_{\mu}$-beam mode is added to utilize all $\bar{\nu}_{\mu}$-CC events from the NuMI beam. Finally, the atmospheric antineutrino interactions are combined with accelerator data to provide the world's most stringent constraints of antineutrino oscillation parameters.

\subsection{Measurements from the $\bar{\nu}_{\mu}$-beam data}

With $3.36 \times 10^{20}$ POT exposure collected in this beam mode, $312 \bar{\nu}_{\mu}$-CC events were expected to seen in the Far Detector provided that there were no neutrino oscillations. In the Far Detector, 226 candidate $\bar{\nu}_{\mu}$-CC events were observed with energies between $0-50 \mathrm{GeV}$. The reconstructed energy spectrum of selected events at the Far Detector and the prediction with no-oscillation hypothesis are showed in Figure 7.1. The binning scheme for these spectra 
was chosen to maximize the sensitivity of the neutrino oscillations: one bin from 0 to $0.5 \mathrm{GeV}, 78$ bins of $0.25 \mathrm{GeV}$ width up to $20 \mathrm{GeV}, 10$ bins of $1 \mathrm{GeV}$ width up to $30 \mathrm{GeV}$, and 10 bins of $2 \mathrm{GeV}$ width up to $50 \mathrm{GeV}$.

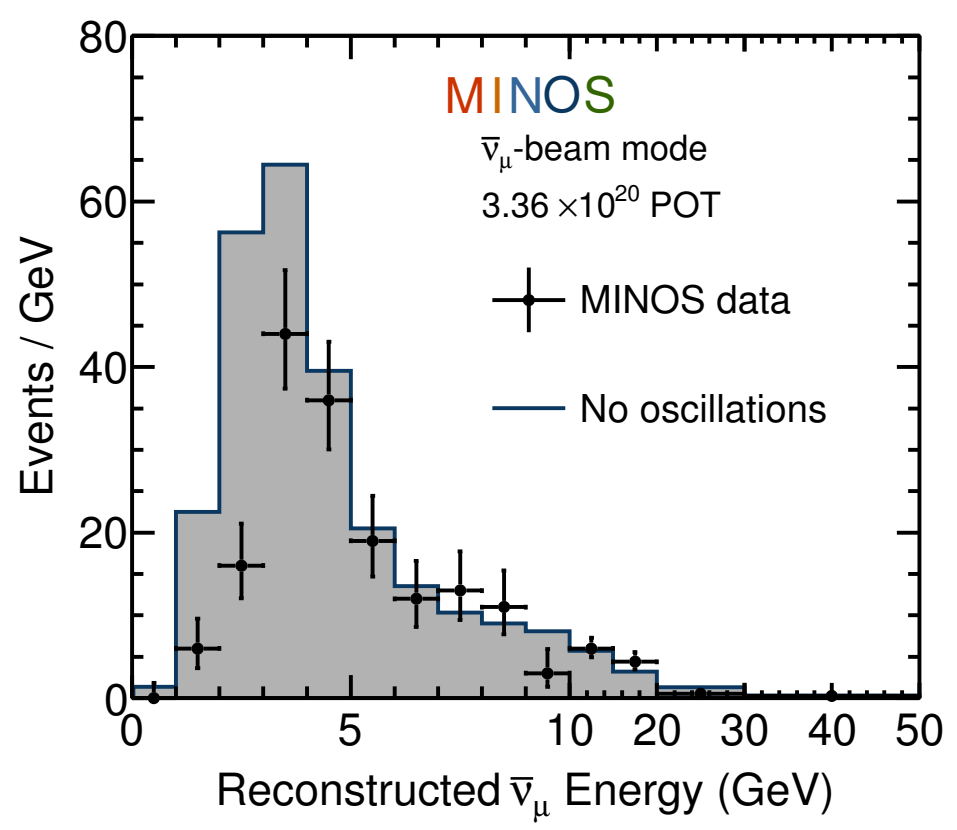

Figure 7.1: The Far Detector $\bar{\nu}_{\mu}$ data and prediction with no oscillations are shown. These correspond to an exposure of $3.36 \times 10^{20}$ POT.

A number of features of the Far Detector selected events were compared to the no-oscillation and oscillated Monte Carlo simulations [134]. Figure 7.2 shows the distribution of the $\mathrm{CC} / \mathrm{NC}$ separation parameter and the charge-tomomentum ratio, while Figure 7.3 presents the reconstructed track and shower energy of the selected events. The features of the selected events agree well with the oscillated Monte Carlo simulation of $\bar{\nu}_{\mu}$ charged-current events. This gives us confidence that the selected events are $\bar{\nu}_{\mu}$-CC events. 

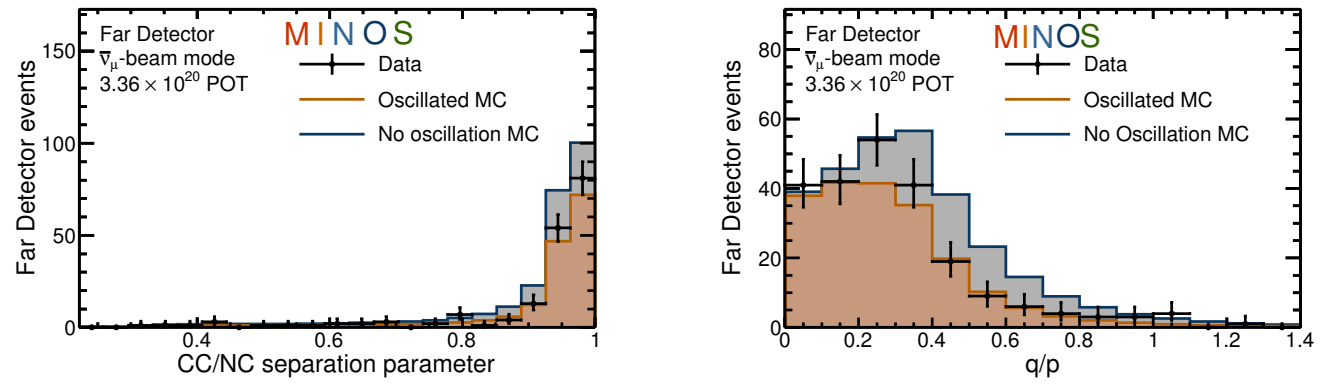

Figure 7.2: Distributions of CC/NC separation parameter (left) and chargeto-momentum ratio (right) of the Far Detector selected $\bar{\nu}_{\mu}$ events. The data (black dots) is compared with no-oscillation (blue line) and oscillated (orange line) MC prediction.
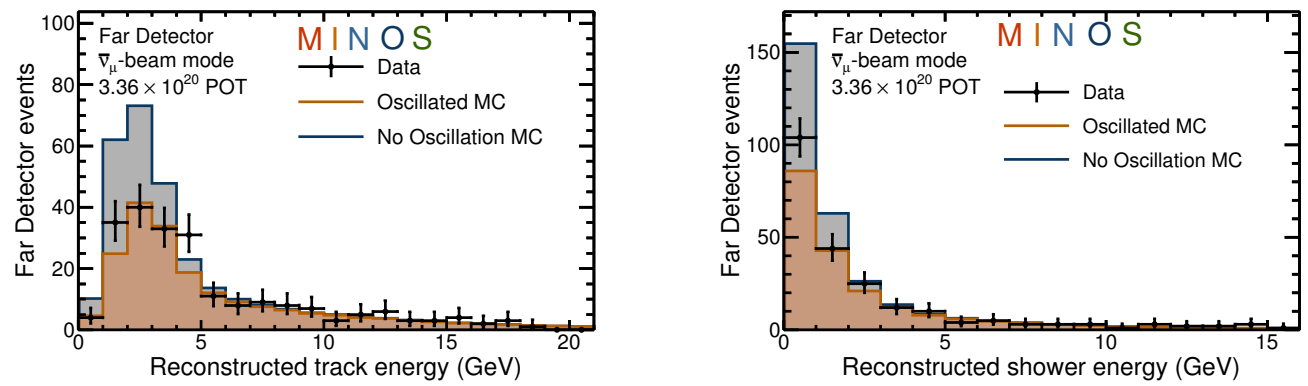

Figure 7.3: Distribution of reconstructed track energy (left) and reconstructed shower energy (right) of the Far Detector selected $\bar{\nu}_{\mu}$ events in Far Detector. The data (black dots) is compared with no-oscillation (blue line) and oscillated (orange line) MC prediction. 


\section{Oscillation results}

A maximum negative log-likelihood approach, introduced in Section 6.6, was adopted to fit the reconstructed energy spectrum of the Far Detector selected events. By assuming that $\bar{\nu}_{\mu}$-CC disappearance is driven by the effective two-flavor model, the best-fit oscillation parameters for considering only statistics yields:

$$
\left|\Delta \bar{m}^{2}\right|=2.64 \times 10^{-3} e V^{2}, \quad \text { and } \quad \sin ^{2}(2 \bar{\theta})=0.95
$$

where $\Delta \bar{m}^{2}$ and $\bar{\theta}$ is an effective mass-squared splitting and the effective mixing angle respectively derived from the three-flavor framework (discussed in Section 1.4.5).

The Far Detector data consists of three independent samples. Since each energy spectrum is represented by 99 bins, the number of degrees of freedom, $N_{d o f}$, is 295 . The $\chi^{2} / N_{d o f}$ value is $287.097 / 295$ for the best-fit point while the $\chi^{2} / N_{\text {dof }}$ for no-oscillation prediction is $353.521 / 295$. This means that the no-oscillation hypothesis is disfavored at $7.7 \sigma$. The neutrino oscillation parameters are reported by MINOS [79] as:

$$
\left|\Delta m^{2}\right|=2.41 \times 10^{-3} e V^{2}, \quad \text { and } \quad \sin ^{2}(2 \theta)=0.95 .
$$

The $\chi^{2} / N_{\text {dof }}$ corresponding to these oscillation parameters is $288.403 / 295$, which is consistent to within $0.6 \sigma$ significance of the antineutrino oscillation parameters. 

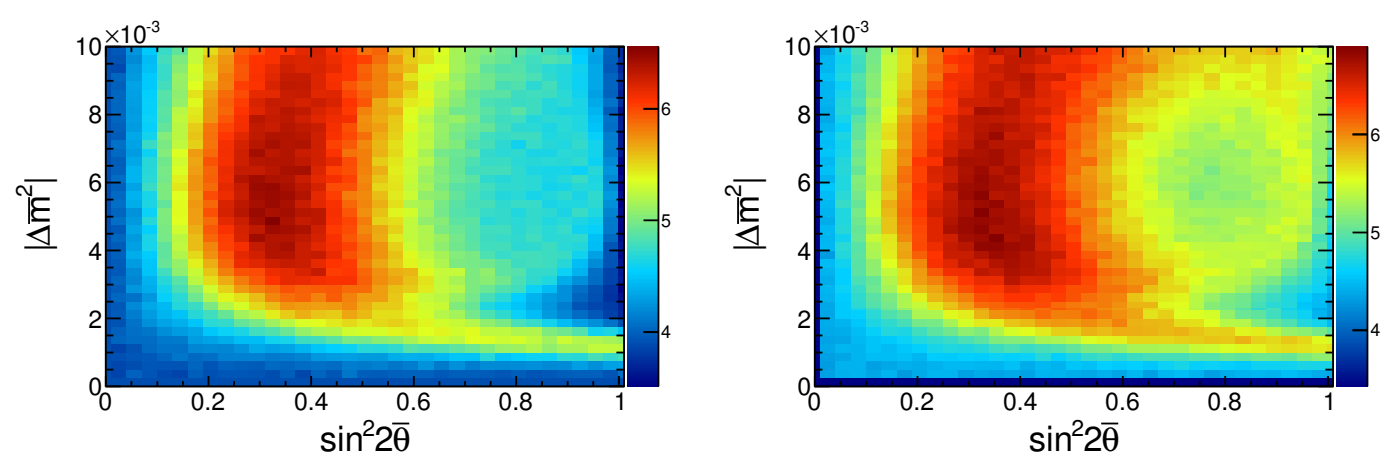

Figure 7.4: The Feldman-Cousins 90\% coverage grid without (left) and with (right) systematic shifts included. The $\mathrm{z}$ value in each bin presents the corrected $\Delta \chi^{2}$ required to cover $90 \%$ experiments. If the experiment is perfectly Gaussian, the value is uniformly 4.61 .

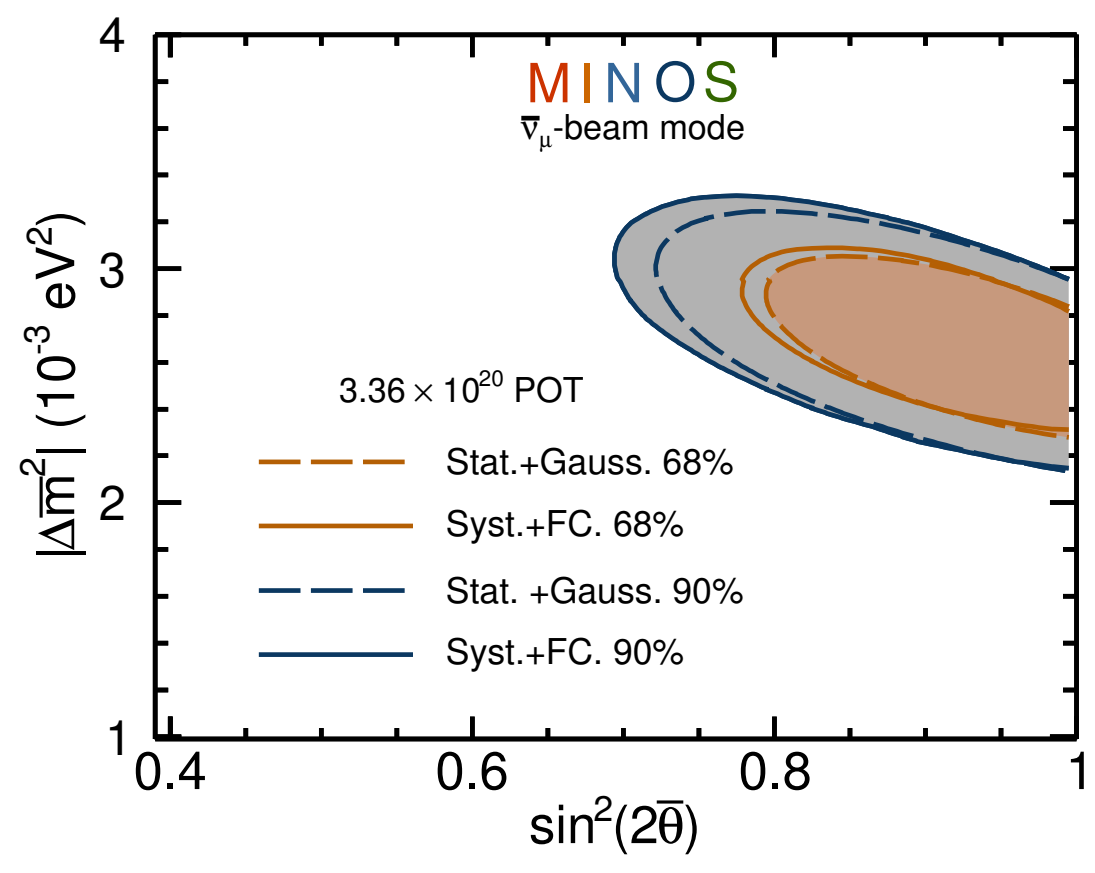

Figure 7.5: The measured confidence contours are compared to the corrected Feldman-Cousins contours with systematic shifts included. 

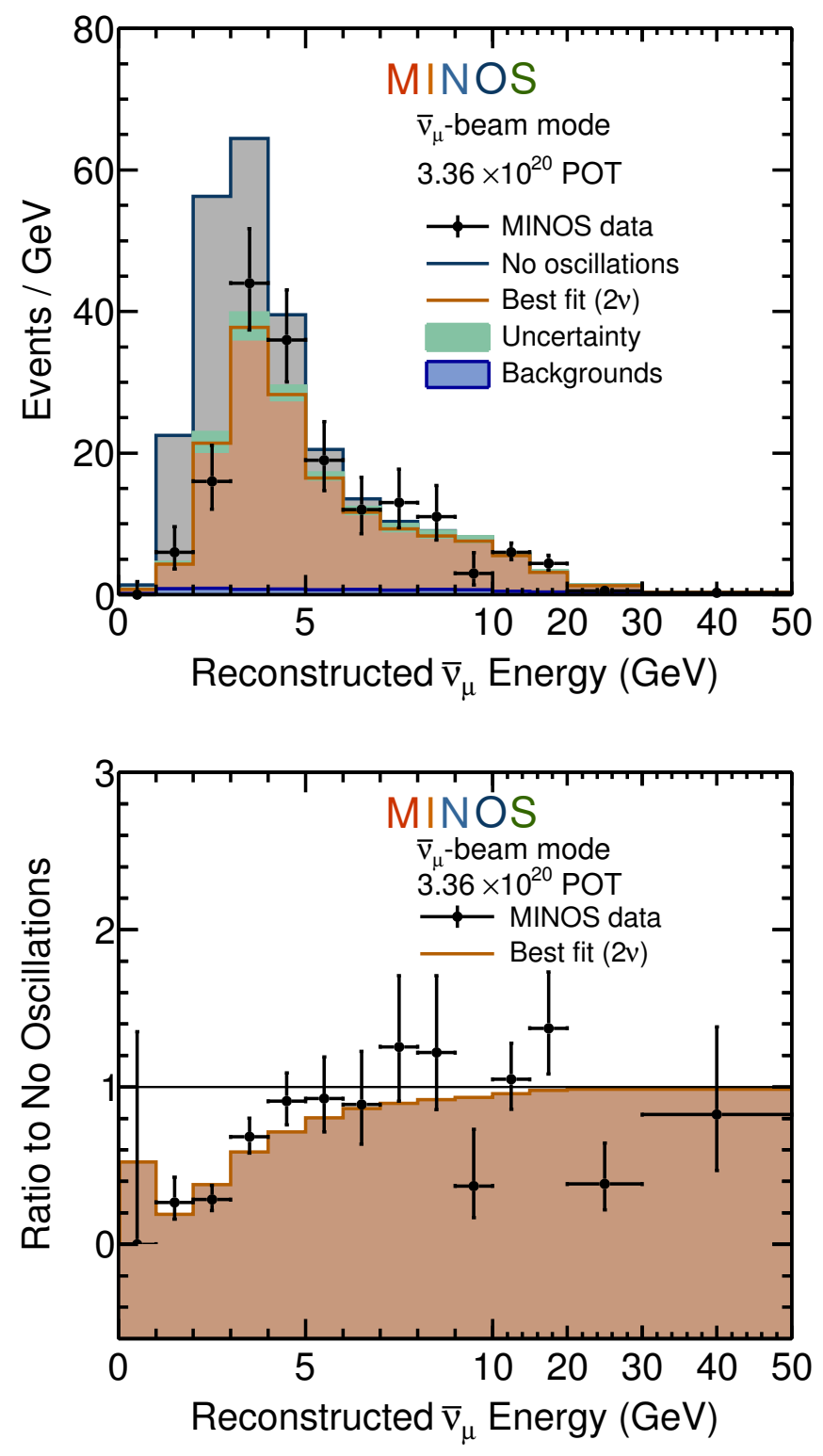

Figure 7.6: Far Detector $\bar{\nu}_{\mu}$ data and predictions with no oscillations and with the best-fit oscillation parameters are in the top. The band around the oscillated prediction represents the total systematic uncertainty. Total background in the oscillated prediction is also displayed. Ratios of Far Detector $\bar{\nu}_{\mu}$ data and best-fit prediction to no oscillations are shown at the bottom. 
Since the value of $\sin ^{2} 2 \bar{\theta}$ is close to a physical boundary, the confidence contours are corrected by applying the Feldman-Cousins method [135]. This method also allows one to incorporate the systematic effects into the final results [136]. Figure 7.4 shows the surfaces of corrected $\Delta \chi^{2}$ with and without systematic shifts applied. These surfaces are then subtracted from the measured confidence contours to get the Feldman-Cousin corrected contours. Figure 7.5 shows the allowed regions with either a statistic fit or a systematic fit with the Feldman-Cousin correction.

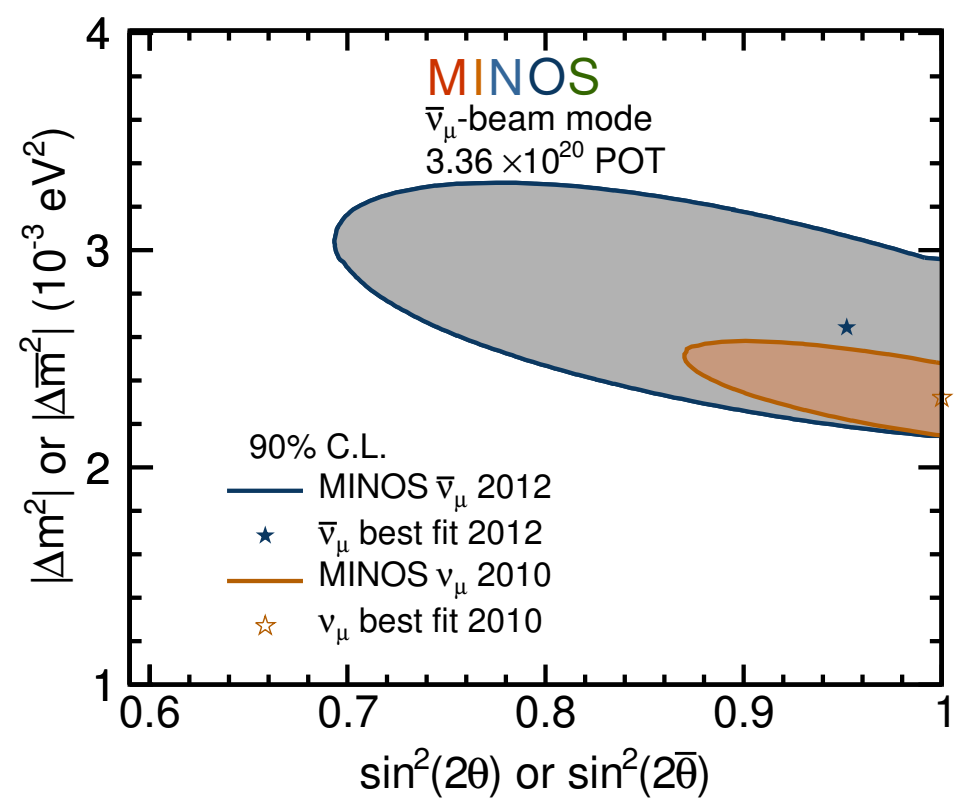

Figure 7.7: The $90 \% 2012$ antineutrino oscillation contour from $\bar{\nu}_{\mu}$-beam mode overlaid with the $2010 \mathrm{CC} \nu_{\mu}$ result. The contours are determined using the Feldman-Cousins method. 
The Far Detector charged current $\bar{\nu}_{\mu}$ data is compared with the best-fit and no-oscillation predictions is shown in Figure 7.6. The result with FeldmanCousins and systematics included is:

$$
\begin{aligned}
& \left.\left.\left|\Delta \bar{m}^{2}\right|=\left[2.64_{-0.25}^{+0.26} \text { (stat. }\right) \pm 0.09 \text { (syst. }\right)\right] \times 10^{-3} \mathrm{eV}^{2} \\
& \left.\sin ^{2}(2 \bar{\theta})=0.95_{-0.10}^{+0.09} \text { (stat. }\right) \pm 0.01 \text { (syst.) }(>0.78 \text { at } 90 \% \text { C.L. }) .
\end{aligned}
$$

The antineutrino oscillation parameters are compared with the MINOS published neutrino result [137] in Figure 7.7. The consistency between the $\bar{\nu}_{\mu}$ and $\nu_{\mu}$ resolves the tension from the previous analysis [2].

To study the effect of systematic uncertainties on the measured oscillation parameters, a number of high statistical Monte Carlo sets are created for faking both the Near Detector and the Far Detector data sets. The fake Far Detector data is weighted with the above best-fit oscillation parameters. For each source of systematics, both the fake Near Detector and Far Detector data sets are fluctuated by $\pm 1 \sigma$. The value of $\pm 1 \sigma$ depends on systematics source as discussed in Section 6.5. The shifted fake Near Detector data set is extrapolated and fits with the shifted Far Detector data set. The difference in fitted oscillation parameters with the shifted fake data sets from the best-fit parameters is taken as a measure of the systematic impact on the measured oscillation parameters. Figure 7.8 shows the shifts to the best-fit oscillation parameters induced by applying individually corresponding systematic uncertainties. Compared to the statistic uncertainty, the systematic impact on measured oscillation parameters is much smaller. 

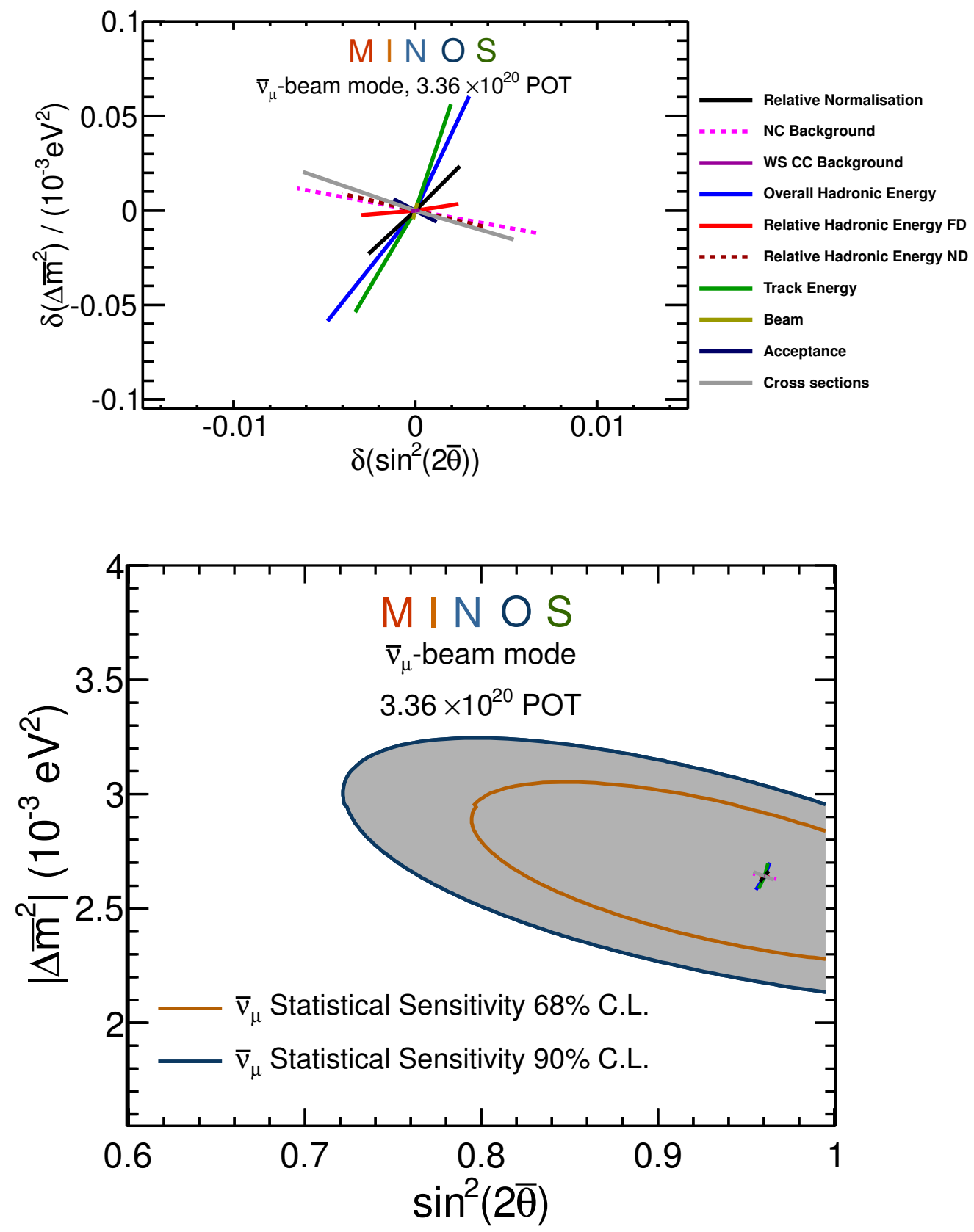

Figure 7.8: The shifts of best-fit values induced by the systematic uncertainties. Evidently, statistic uncertainty is dominant in measurement of antineutrino oscillation parameters. 


\subsection{Measurements from both $\bar{\nu}_{\mu}$-beam and $\nu_{\mu}$-beam data}

To increase the sensitivity of measuring antineutrino oscillation parameters, combining $\bar{\nu}_{\mu}$-CC disappearance from two beam modes was proposed [138]. Based on the Far Detector exposure of $3.36 \times 10^{20} \mathrm{POT}$ in $\bar{\nu}_{\mu}$-beam mode and $10.71 \times 10^{20} \mathrm{POT}$ in $\nu_{\mu}$-beam mode ${ }^{2}, 556 \bar{\nu}_{\mu}$-CC events are expected provided no-oscillation hypothesis. We observed a total of 442 events in the Far Detector, which includes 216 events in the $\nu_{\mu}$-beam data and 226 events in the $\bar{\nu}_{\mu}$-beam data. Figure 7.9 shows the Far Detector data and no-oscillation prediction either from the $\nu_{\mu}$-beam data or from the combined data set.

\footnotetext{
${ }^{2}$ We did not use the $0.21 \times 10^{20}$ POT high-energy beam data, for $\bar{\nu}_{\mu}$ measurements.
} 


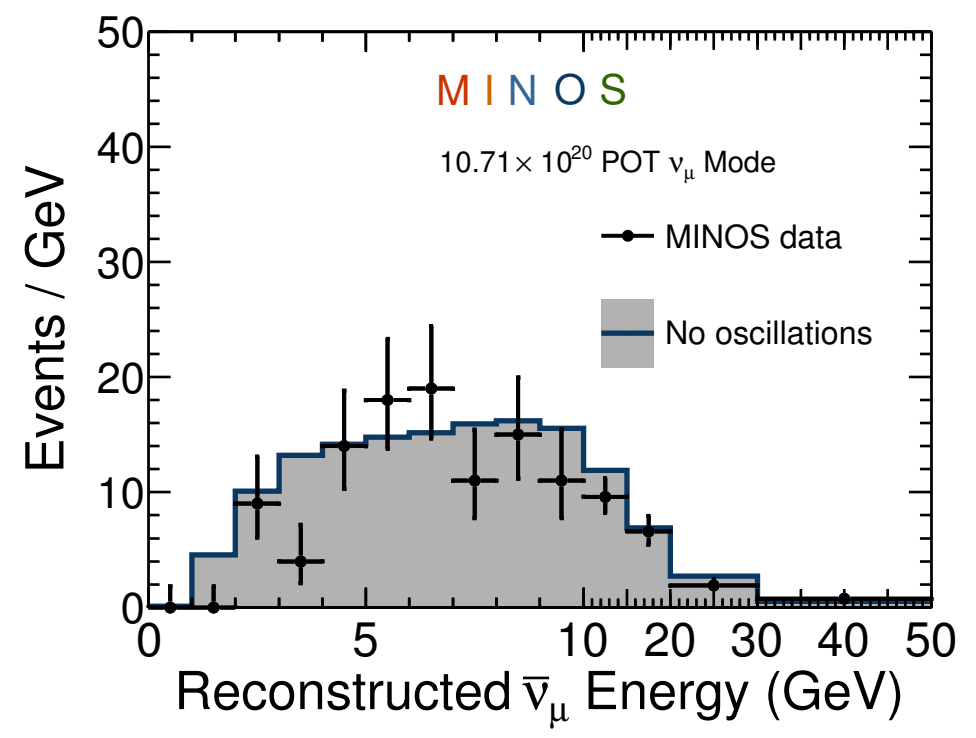

(a)

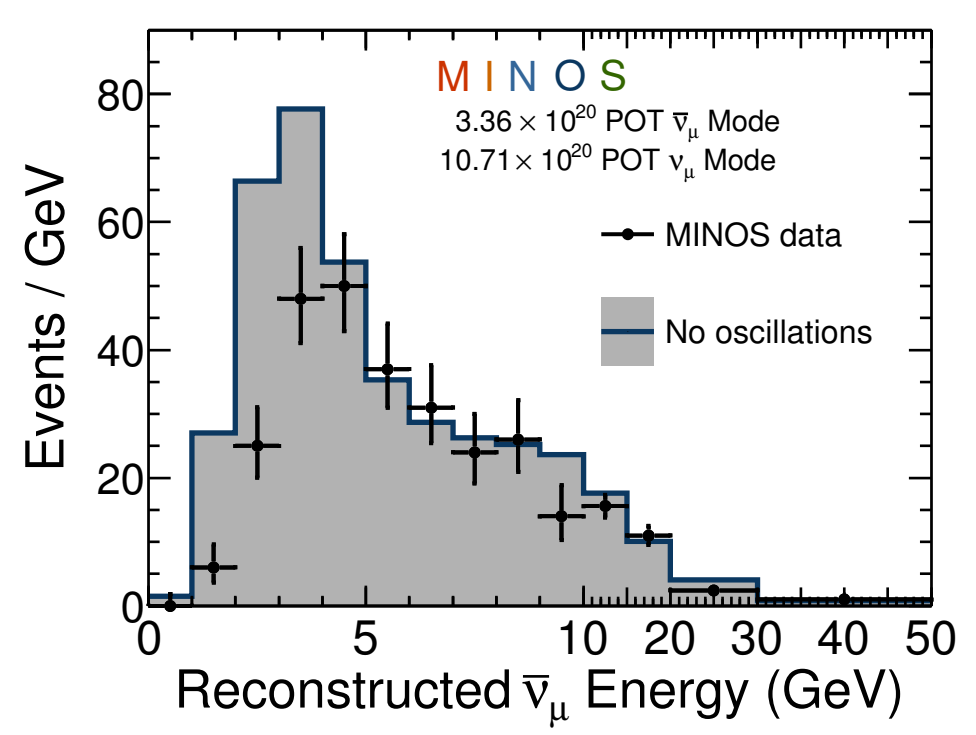

(b)

Figure 7.9: The Far Detector $\bar{\nu}_{\mu}$-CC energy spectra in $\nu_{\mu}$-beam mode (left) and with combined data (right) are shown with the corresponding no-oscillation predictions. 


\section{Oscillation results}

To perform an analysis with a combined data set, we can not simply add log-likehood confident contours because of systematic correlations among data sets. To deal with this, the systematic uncertainties are included as nuisance parameters in the oscillation parameter fitting [58]. For the measurement of $\bar{\nu}_{\mu}$-CC disappearance in the combined $\bar{\nu}_{\mu}$-beam and $\nu_{\mu}$-beam data set, four of the largest systematic uncertainties are incorporated into the fit. They include systematic uncertainties of overall normalization of data exposure, the neutral-current background, the track energy, and the shower energy, all detailed in Section 6.5. Since the systematics are included in the fit, the systematic and statistic uncertainties of the oscillation parameters are combined into one single value. Assuming the two-flavor neutrino oscillation hypothesis as in Section 7.2, the best-fit oscillation parameters, obtained from the maximum negative log-likelihood approach, yields:

$$
\begin{aligned}
& \left|\Delta \bar{m}^{2}\right|=\left[2.62_{-0.24}^{+0.26}\right] \times 10^{-3} e V^{2} \\
& \sin ^{2}(2 \bar{\theta})=0.97_{-0.10}^{+0.03}(>0.8 \text { at } 90 \% \text { C.L. }) .
\end{aligned}
$$

Figure 7.10 shows the energy spectra with null-oscillation and best-fit predictions either with the $\bar{\nu}_{\mu}$ data set from $\nu_{\mu}$-beam or with the combined data set. 

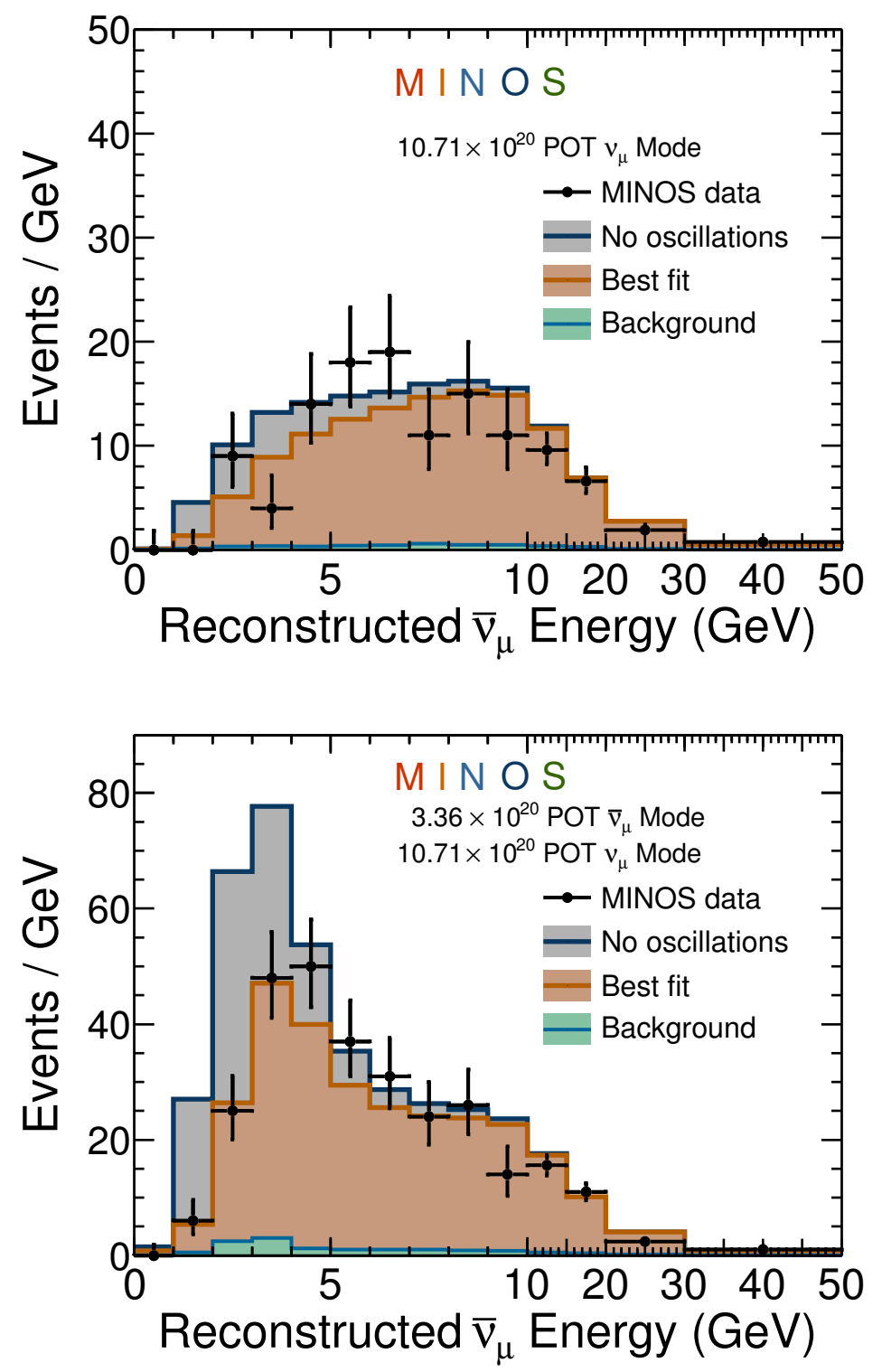

Figure 7.10: The Far Detector $\bar{\nu}_{\mu}$ charged-current energy spectra in $\nu_{\mu}$-beam mode (left) and with combined data (right) is shown with the predictions of no oscillation and with best-fit oscillation parameters. 


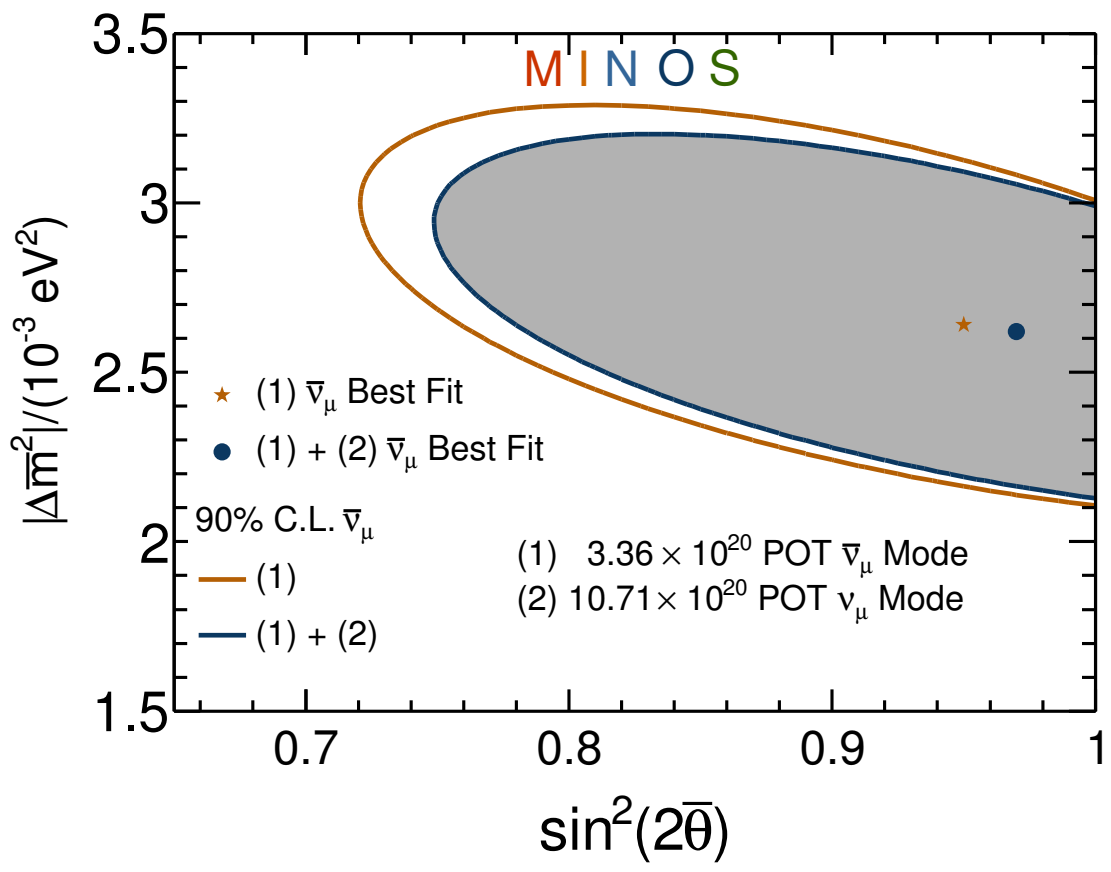

Figure 7.11: The data contour of combining the $\bar{\nu}_{\mu}$-CC data in $\overline{\nu_{\mu}}$-beam and $\nu_{\mu}$-beam modes in comparison with the contour using data in $\bar{\nu}_{\mu}$-beam mode only.

Figure 7.11 shows the comparison of confidence contours when using only the $\bar{\nu}_{\mu}$-beam data and when using the combined data. The measurement with combined data yields an additional constraint on the oscillation parameters.

The impact of systematic uncertainties on the best-fit oscillation parameters are studied with a similar approach as introduced in Section 7.2. Figure 7.12 shows the shifts on the best-fit values induced by four main systematics. The uncertainties of best-fit oscillation parameters is undoubtedly dominated by the statistical uncertainty. 

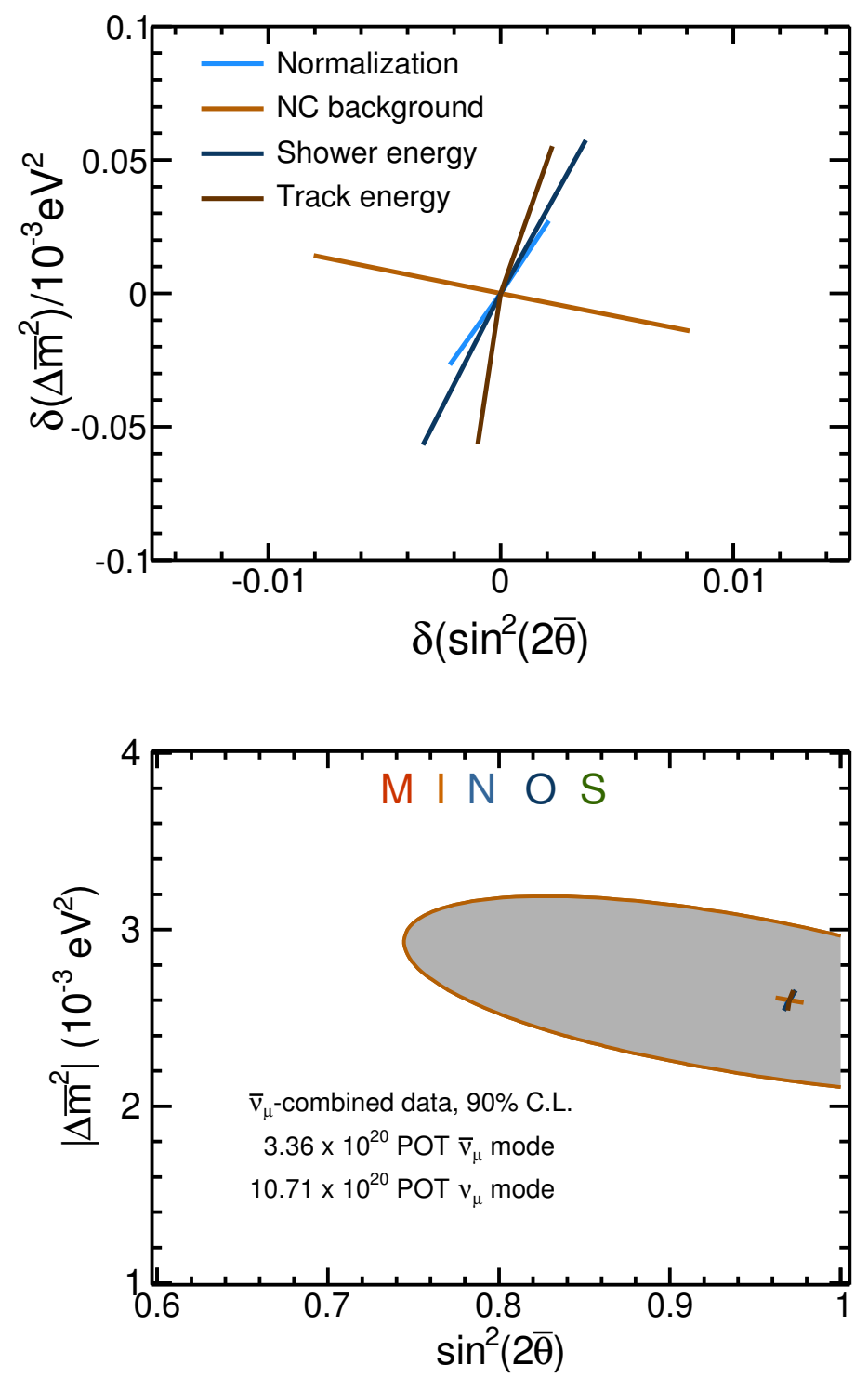

Figure 7.12: The shifts on the best-fit oscillation parameters are induced by applying four main systematic uncertainties to the Monte Carlo simulation when fitting with combined $\bar{\nu}_{\mu}$ data from the exposure of $3.36 \times 10^{20} \mathrm{POT}$ $\bar{\nu}_{\mu}$-beam mode and $10.71 \times 10^{20} \mathrm{POT} \nu_{\mu}$-beam mode. 


\subsection{Measurements from combined accelerator and at- mospheric data}

The atmospheric $\bar{\nu}_{\mu}$-CC events in the MINOS Far Detector was collected for 2553 live-days (corresponding to 37.9 kton-years) of data. A total of $268 \bar{\nu}_{\mu}$-CC events, which include 173 contained-vertex events and 95 neutrino-induced rock events were observed. The expected number of events for a no oscillation hypothesis is $219 \pm 31$ for contained-vertex events and $112 \pm 26$ neutrino-induced rock events respectively. The measurements of atmospheric neutrino and antineutrino interactions in the MINOS Far Detector were reported in [127]. Figure 7.13 shows the distributions of Far Detector atmospheric $\bar{\nu}_{\mu}$-CC data, the null-oscillation prediction, and the best-fit oscillated prediction, along with the background of cosmic-rays and neutral-current events.

Inspired by the success of measurements from the combined beam data set, as described in Section 7.3, measurements from the complete MINOS accelerator and atmospheric $\bar{\nu}_{\mu}$ data set were proposed to get the most stringent constraints on the antineutrino oscillation parameters. The addition of loglikelihood contours first came up [139]. However, this simple addition did not account for systematic correlation among data sets. A fitting framework was developed to incorporate systematic uncertainties as nuisance parameters [140]. 

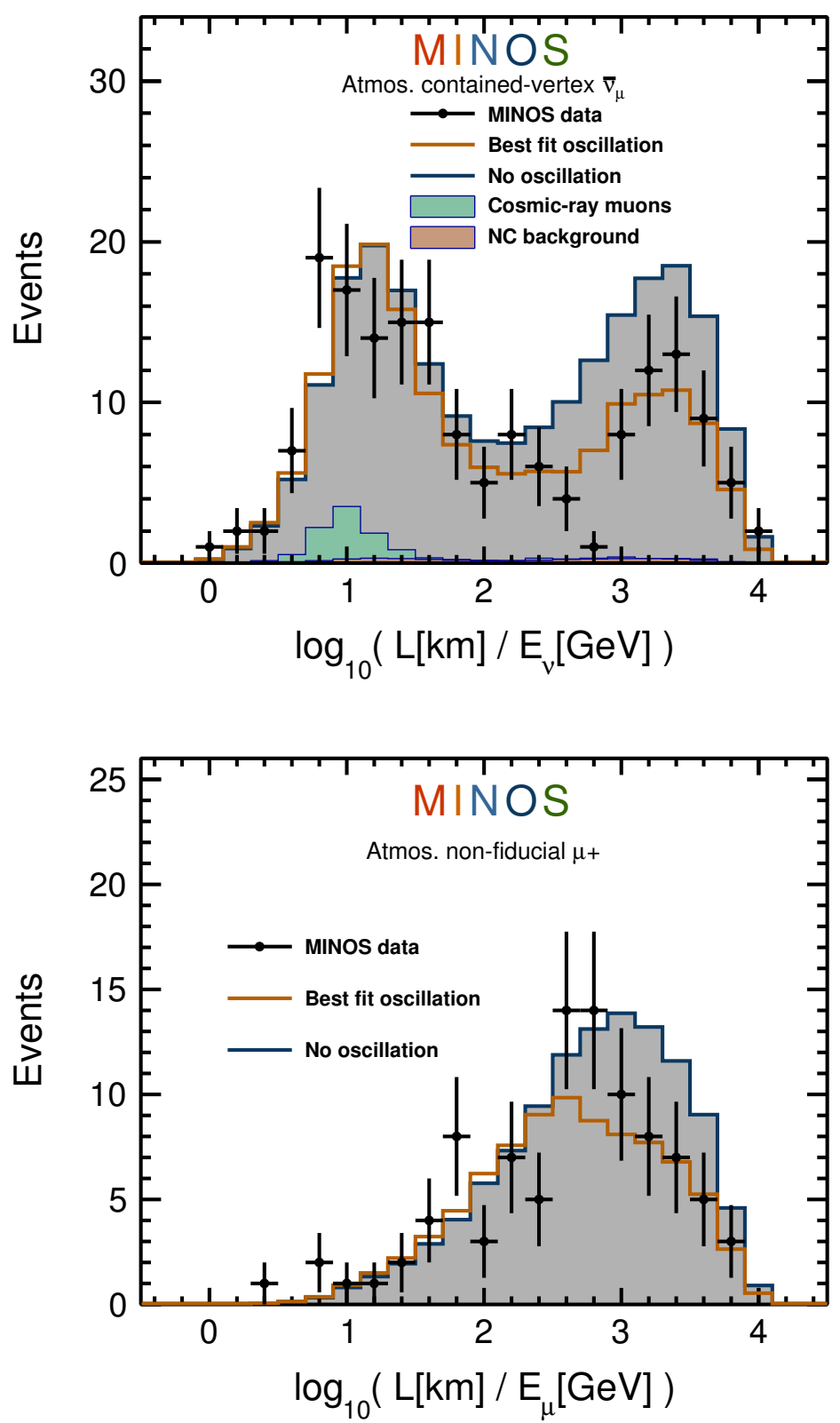

Figure 7.13: The distribution of reconstructed $\log _{10}(L / E)$ for atmospheric contained-vertex $\bar{\nu}_{\mu}$ (left) and neutrino-induced rock-anti muon (right). 


\section{Oscillation results}

In this measurement, the neutrino oscillation parameters are fixed at $\left|\Delta m^{2}\right|=2.41 \times 10^{-3} \mathrm{eV}^{2}$ and $\sin ^{2} 2 \theta=0.95$. This best-fit point was measured by the $\nu_{\mu}$-CC disappearance in $10.71 \times 10^{20} \mathrm{POT}$ of a $\nu_{\mu}$-beam mode. Assuming the $\bar{\nu}_{\mu}$-CC survival probability described by the two-flavor model, the oscillation parameter, obtained from the maximum negative log-likelihood approach, yields:

$$
\begin{aligned}
& \left|\Delta \bar{m}^{2}\right|=\left[2.50_{-0.29}^{+0.23}\right] \times 10^{-3} \mathrm{eV}^{2}, \\
& \sin ^{2}(2 \bar{\theta})=0.97_{-0.08}^{+0.03}(>0.83 \text { at } 90 \% \text { C.L. }) .
\end{aligned}
$$

These results provide the world's most stringent constraint to date on the antineutrino oscillation parameters. Figure 7.14 shows the profile of the negative $\Delta$ log-likelihood for the fit to all $\bar{\nu}_{\mu}$ selected events for $\Delta \bar{m}^{2}$ and for $\sin ^{2} 2 \bar{\theta}$.
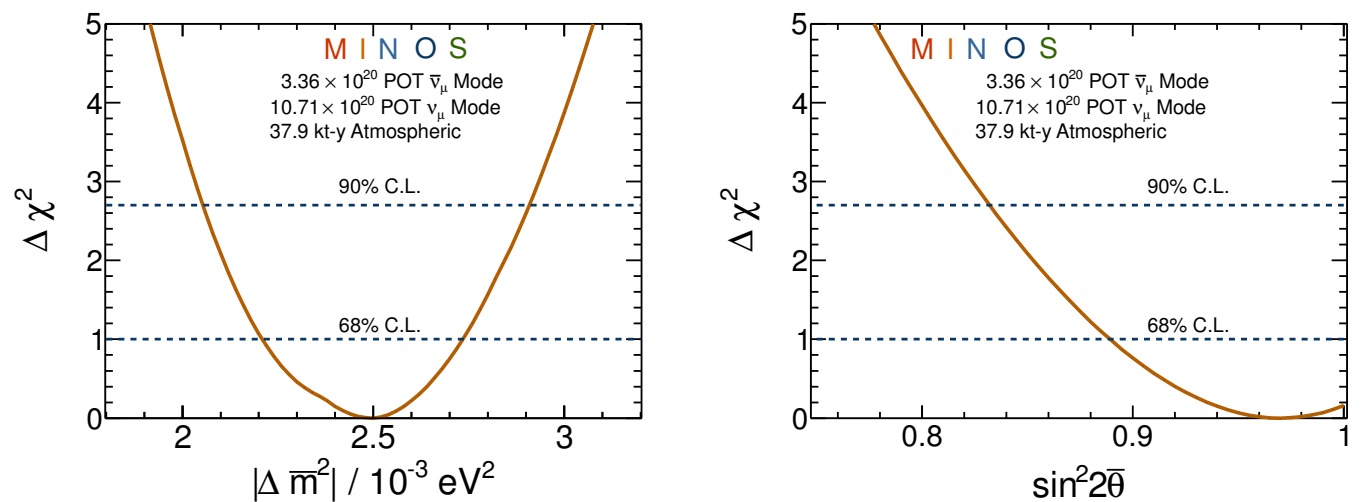

Figure 7.14: The negative $\Delta \log$ likelihood contour for the two parameter fit to all MINOS $\bar{\nu}_{\mu}$-CC data for $\Delta \bar{m}^{2}$ and for $\sin ^{2} 2 \bar{\theta}$ are shown. 
The best-fit values of systematic uncertainties, which were treated as the nuisance parameters, are shown in Figure 7.15. Almost all systematic uncertainties yield shifts that are within $1 \sigma$ of the best-fit point.

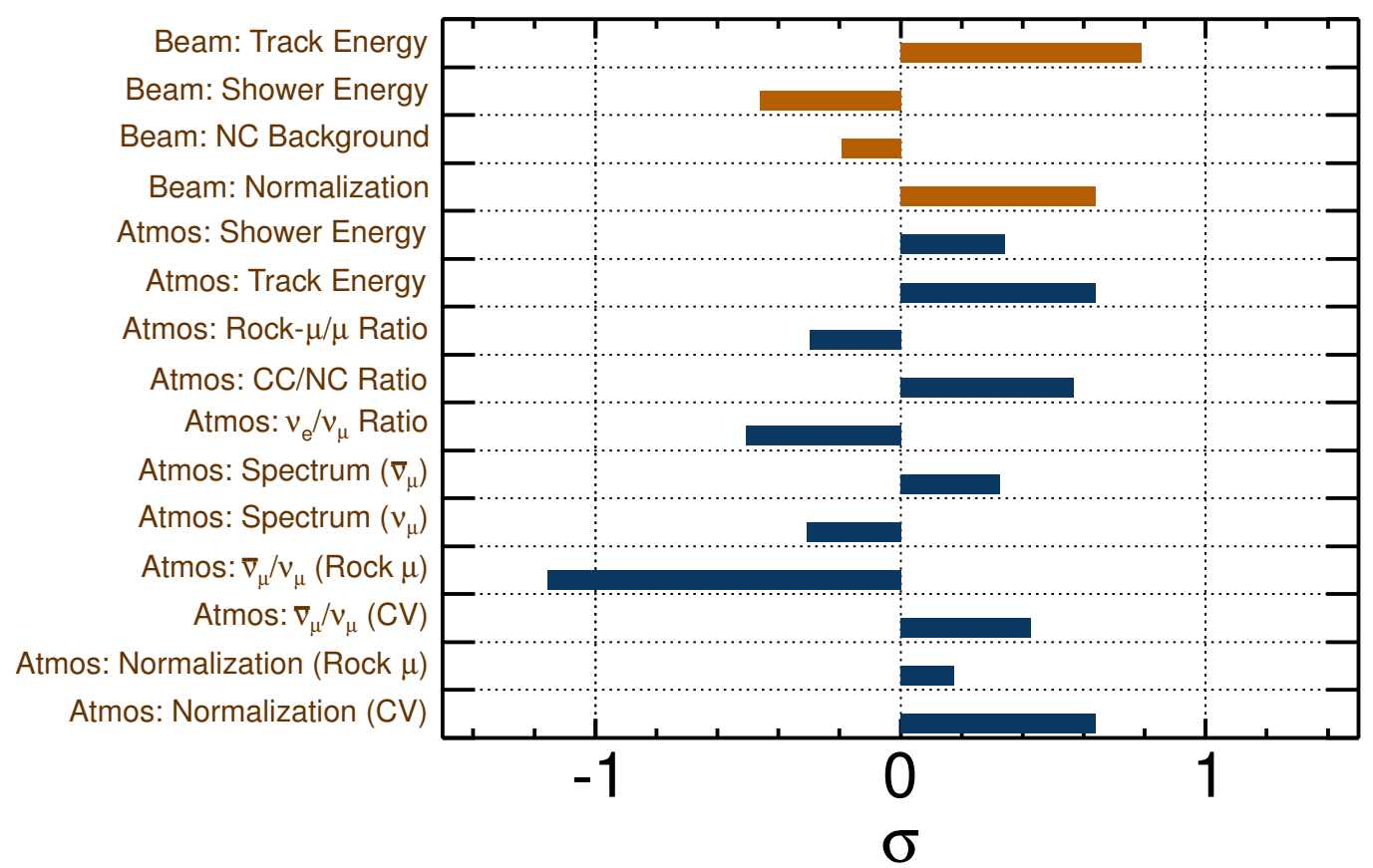

Figure 7.15: Values of the best-fit systematic uncertainties resulted by the fitting the complete accelerator and atmospheric $\bar{\nu}_{\mu}$ data set, where CV means contained vertex event. 


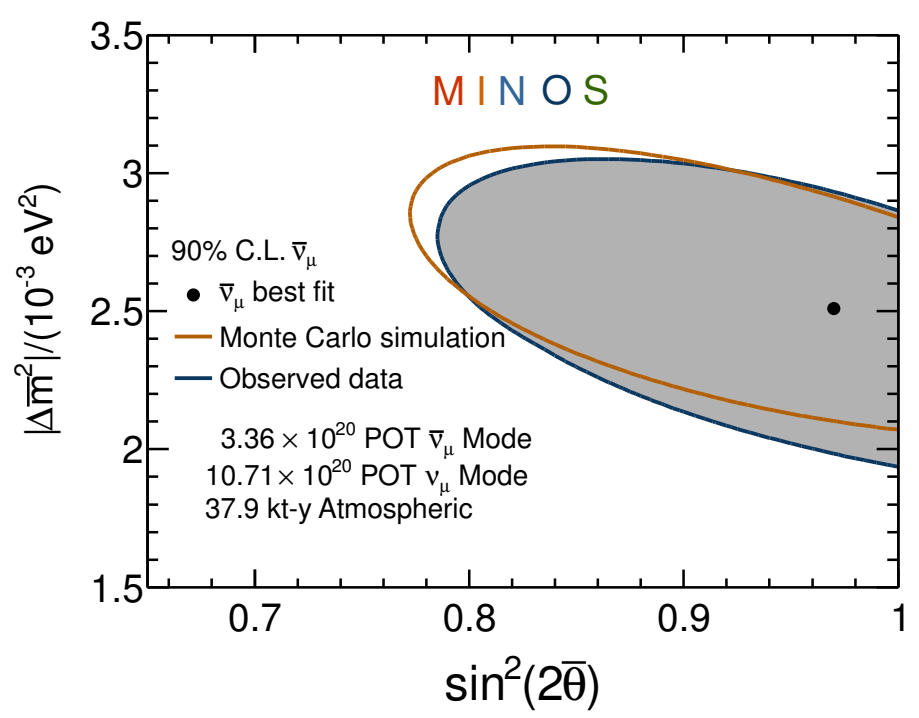

(a) Data vs MC prediction

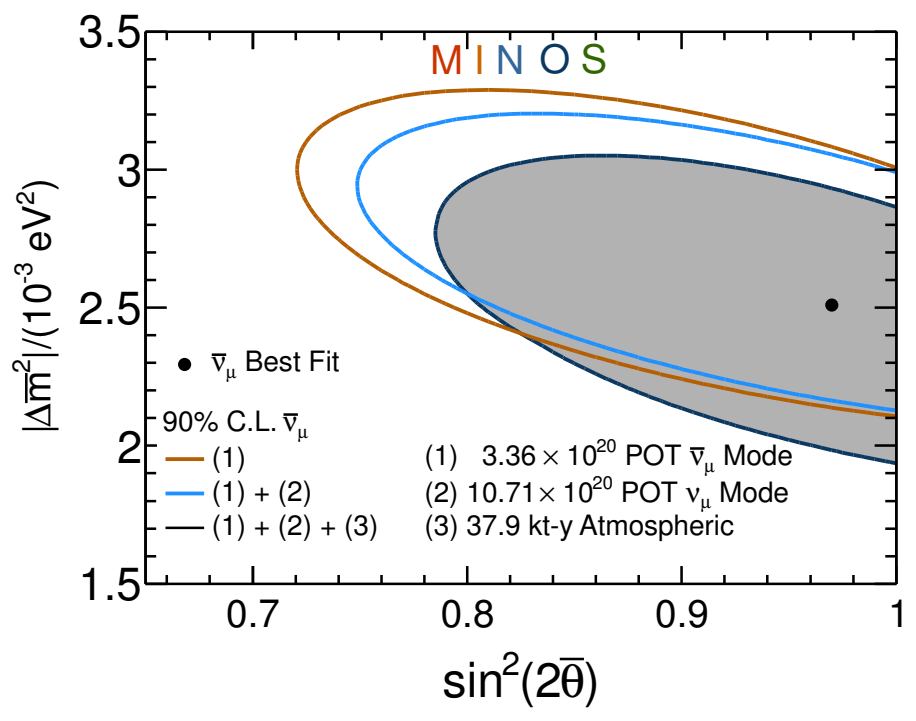

(b) Progression of $90 \%$ C.L. $\bar{\nu}_{\mu}$ contours

Figure 7.16: The comparison between the data contour and the predicted sensitivity contour at our best-fit point (left). The three $90 \%$ confidence level contours (right) for the anti-neutrino oscillation parameters from MINOS using the $\bar{\nu}_{\mu}$-beam data, combination of the $\bar{\nu}_{\mu}$-beam and $\nu_{\mu}$-beam data, and the complete accelerator and atmospheric data are compared. 
The confidence region measured by observed data is compared to the Monte Carlo sensitivity prediction, as shown in Figure 7.16a. Figure 7.16b shows the progression of $90 \%$ C.L contours of $\bar{\nu}_{\mu}$ oscillation parameters when adding gradually three MINOS $\bar{\nu}_{\mu}$-CC data sets. The constraint on the mixing angle $\bar{\theta}$ is substantially better while the improvement on the mass-squared mixing $\Delta \bar{m}^{2}$ is comparatively small.

To demonstrate that there is no bias in the best-fit oscillation parameters and systematic uncertainties, we generate a set of 1000 pseudoexperiments from the combined accelerator and atmospheric neutrino Monte Carlo samples. The data in each pseudo-experiment is created by applying Poisson fluctuations to each bin of the Far Detector predicted spectrum. For each pseudo-experiment, a set of systematic uncertainties is randomly selected from a Gaussian distribution with a mean of zero and a width equal to the $1 \sigma$ of systematic uncertainty. Each pseudo-experiment is then fitted by the same procedure as applied to the real data. The distributions of the best-fit systematic uncertainties are shown in Figure 7.17. These distributions all are well matched by the Gaussian distribution of zero mean value and a width close to unity. This assures that there is no bias in fitting the antineutrino oscillation parameters. Figure 7.18 shows the distribution of log-likelihood returned by fitting pseudo-experiments in comparison to the log-likelihood from the fit of real data. The fraction of pseudo-experiments with a better best-fit than the real data is $60 \%$. Approximately $96 \%$ of the best-fit points occur inside the predicted $90 \%$ C.L. contour. 

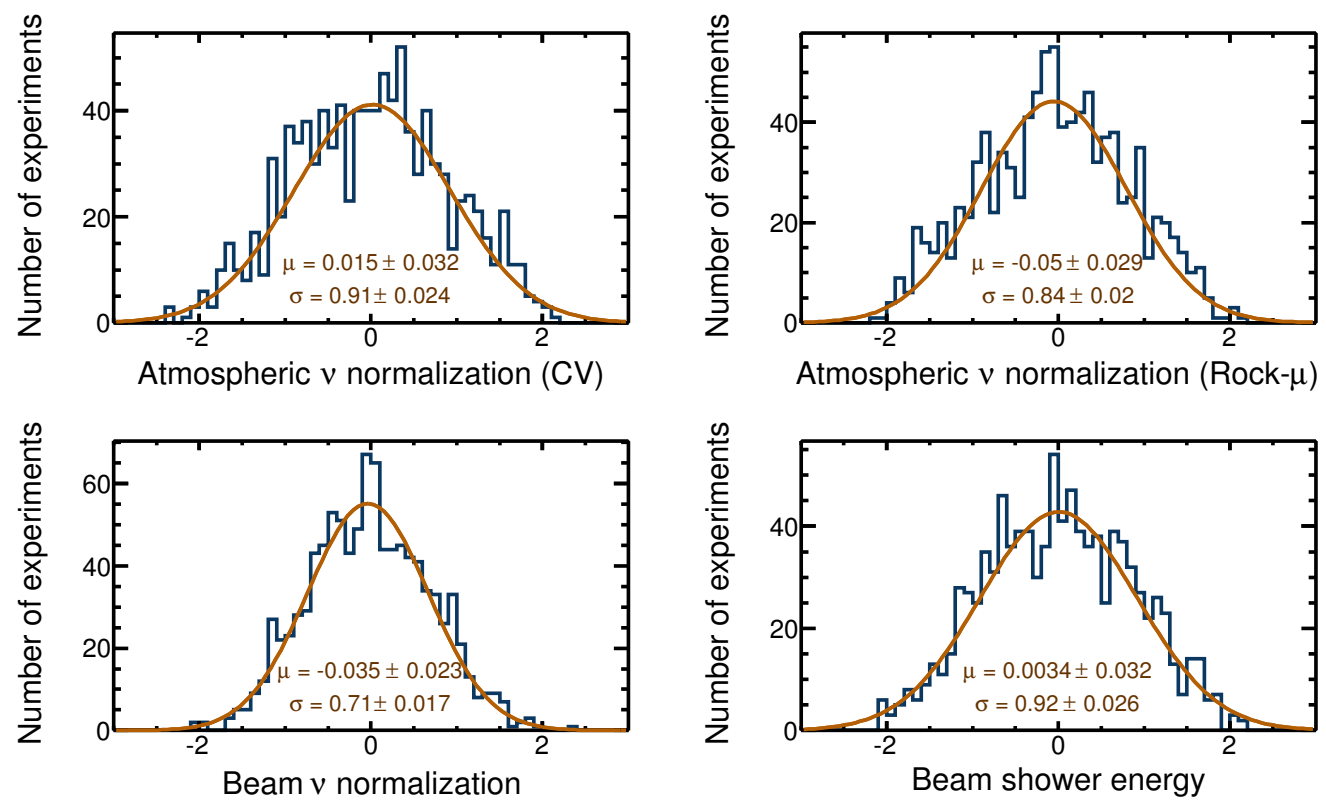

Figure 7.17: The distribution of the major systematics obtained by a two parameter fit on 1000 pseudo-experiments. These distributions are well described by the Gaussian distribution.
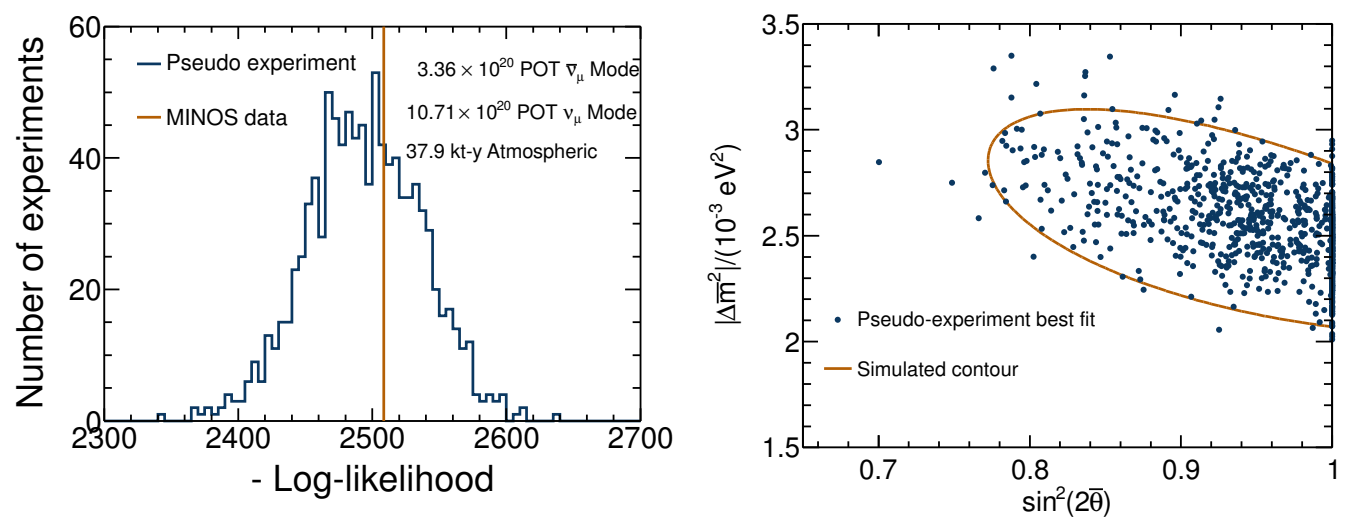

Figure 7.18: Distribution of the negative log-likelihood (left) returned from the fit on 1000 pseudo-experiment in comparison to the real data. The best-fit oscillation parameters (right) of these pseudo-experiments are overlaid with the expected $90 \%$ C.L. contour. 
To study the effect of systematic uncertainties on the best-fit for the antineutrino oscillation parameters, a set of systematically shifted fake data is generated. For each fake data sample, a considered systematic parameter is shifted by $\pm 1 \sigma$. We redo the fit for these systematically shifted samples with the similar procedure as the real data and estimate how much the bestfit of oscillation parameters change. Figure 7.19 shows the shifts of oscillation parameters resulted by shifting systematic parameters. The dominant ones are the atmospheric normalization uncertainties for both contained-vertex events and for rock-muon events. 


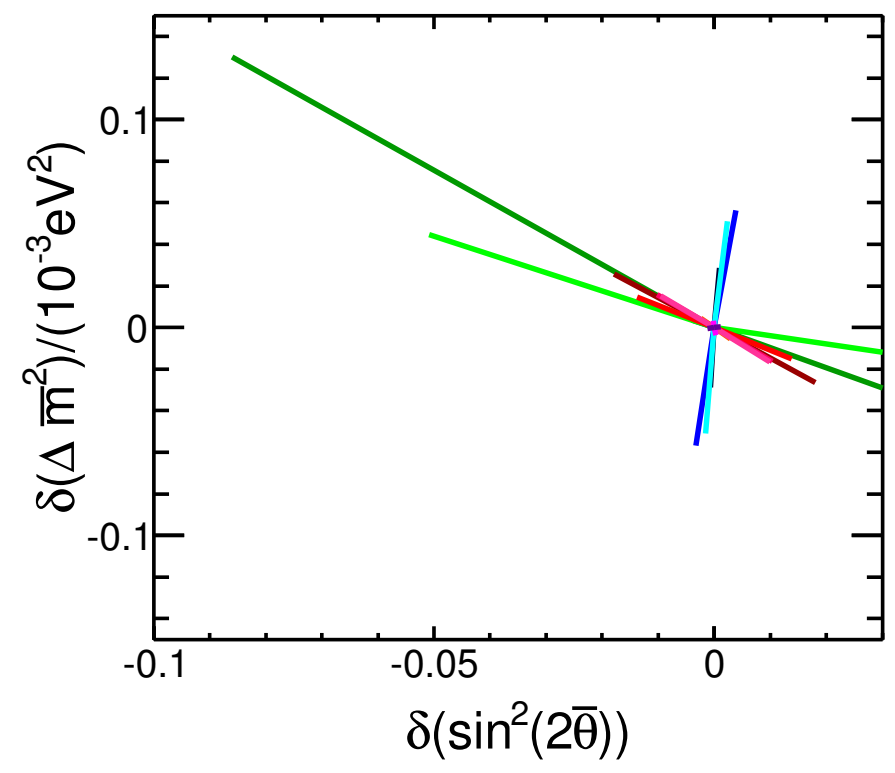

Accelerator

- Normalization

NC Background

Shower Energy

Track Energy

Atmospheric

Normalization (CV)

Normalization (Rock $\mu$ )

$v_{\mathrm{e}} / v_{\mu}$ Ratio

NC/CC Ratio

Up/Down Ratio

Charge Ratio (CV)

Charge Ratio (Rock $\mu$ )

Spectrum $\left(v_{\mu}\right)$

Spectrum $\left(\nabla_{\mu}\right)$

Track Energy

Shower Energy

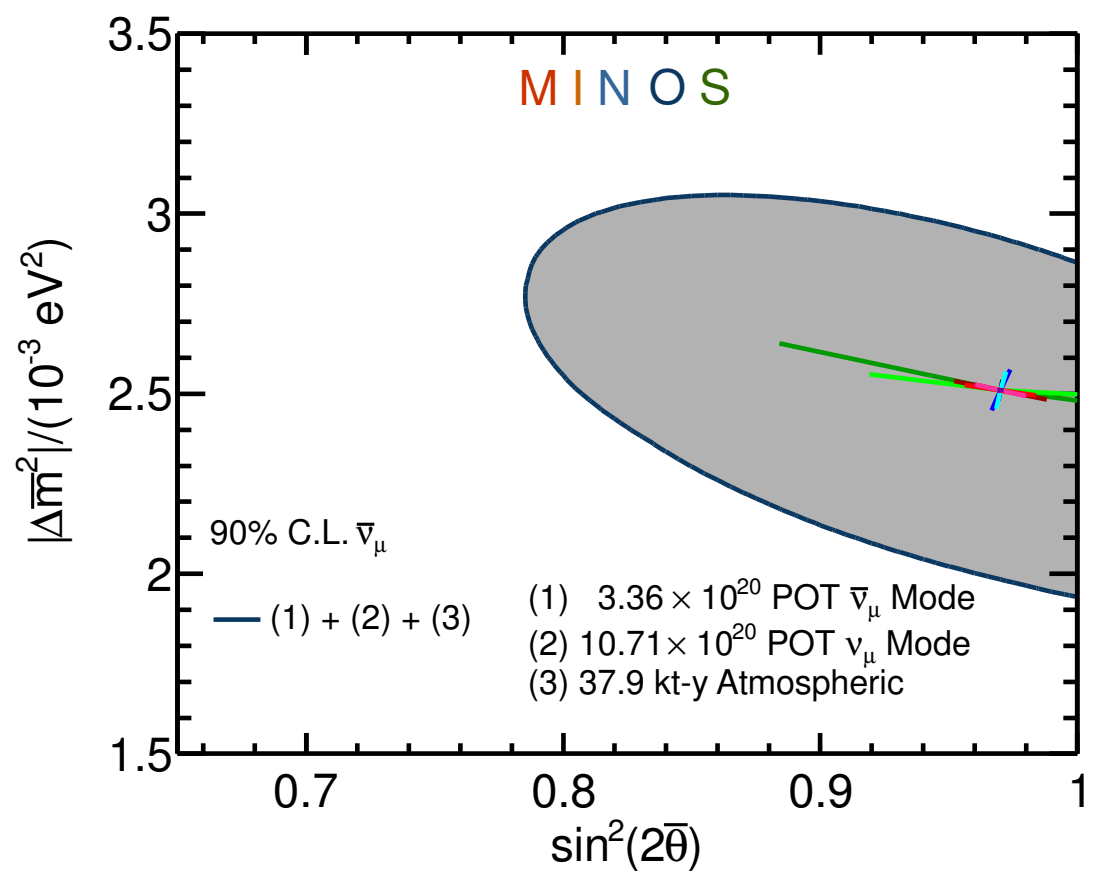

Figure 7.19: The effect of systematic uncertainties on the best-fit antineutrino oscillation overlaid with the $90 \%$ C.L. contour measured by the complete MINOS accelerator and atmospheric data set. 


\subsection{CPT-invariant testing in $\nu_{\mu}$ and $\bar{\nu}_{\mu}$ oscillations}

All the neutrino and antineutrino interactions collected by the MINOS detectors from the accelerator and atmospherics are combined in the same framework. This permits one to make the fit simultaneously for neutrino and antineutrino events. In other words, four parameters, $\left|\Delta m^{2}\right|, \sin ^{2} 2 \theta,\left|\Delta \bar{m}^{2}\right|$, and $\sin ^{2} 2 \bar{\theta}$, can be floated in the fit. Four systematic uncertainties for the accelerator data are treated correlatively between the $\bar{\nu}_{\mu}$ and $\nu_{\mu}$ events. The best-fit neutrino and antineutrino oscillation parameters, returned from 4parameter fit, yields:

$$
\begin{aligned}
& \left|\Delta \bar{m}^{2}\right|=\left[2.50_{-0.24}^{+0.24}\right] \times 10^{-3} \mathrm{eV}^{2}, \quad \sin ^{2}(2 \bar{\theta})=0.975_{-0.085}^{+0.025}(>0.835 \text { at } 90 \% \text { C.L. }), \\
& \left|\Delta m^{2}\right|=\left[2.38_{-0.9}^{+0.11}\right] \times 10^{-3} \mathrm{eV}^{2}, \quad \sin ^{2}(2 \theta)=0.955_{-0.039}^{+0.037}(>0.887 \text { at } 90 \% \text { C.L. }) .
\end{aligned}
$$

Figure 7.20 shows the $90 \%$ C.L. contours obtained from the measurements of the $\nu_{\mu}$ and $\bar{\nu}_{\mu}$ disappearances with the complete MINOS accelerator and atmospheric data. Compared to the $\bar{\nu}_{\mu}$ allowed region in which the $\nu_{\mu}$ oscillation parameters are fixed, the contour with floated $\nu_{\mu}$ oscillation parameters is smaller due to the minimum negative log-likelihood over $\left|\Delta m^{2}\right|, \sin ^{2} 2 \theta$ and due to additional constraints from the systematic correlations. 


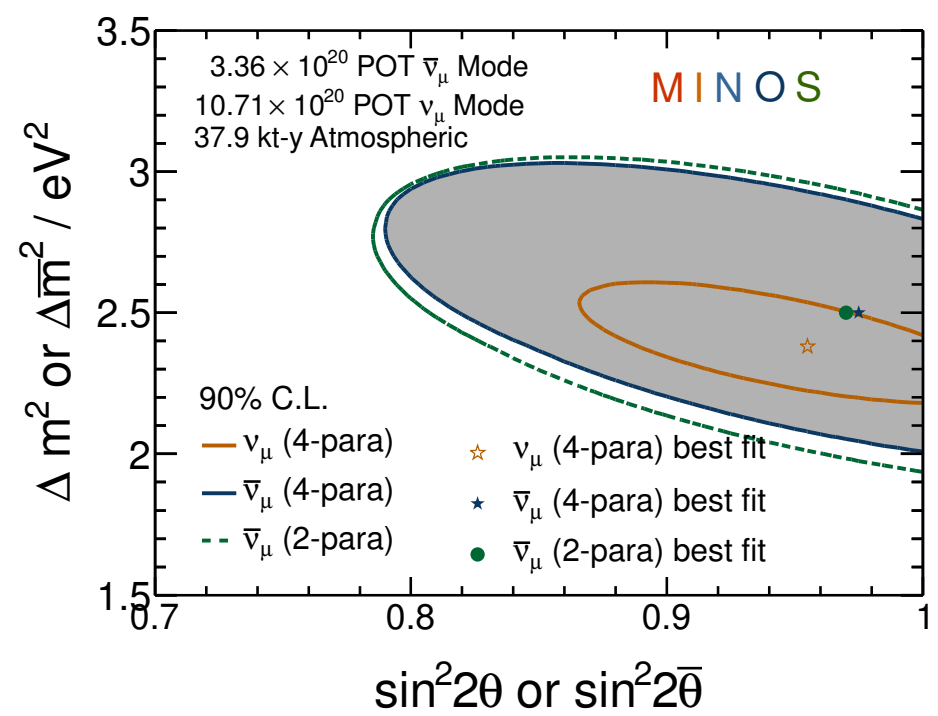

Figure 7.20: The 90\% C.L. contours for the neutrino and antineutrino oscillation parameters measured by the complete MINOS accelerator and atmospheric data set.

To obtain the allowed region for mass-squared splittings of neutrino and antineutrino events, we minimize the negative log-likelihood values in each point of $\left|\Delta m^{2}\right|-\left|\Delta \bar{m}^{2}\right|$ parameter space with respect to the mixing angle $\sin ^{2} 2 \theta$ and $\sin ^{2} 2 \bar{\theta}$. Figure 7.21 shows the $68 \%$ and $90 \%$ C.L. contours for the difference between the mass-squared splittings of neutrinos and antineutrinos. The figure illustrates the good agreement between the neutrino and anti-neutrino mass-squared splittings. The difference between the antineutrino and neutrino mass-squared splittings is measured to be:

$$
\left|\Delta \bar{m}^{2}\right|-\left|\Delta m^{2}\right|=0.13_{-0.25}^{+0.23} \times 10^{-3} e V^{2} .
$$



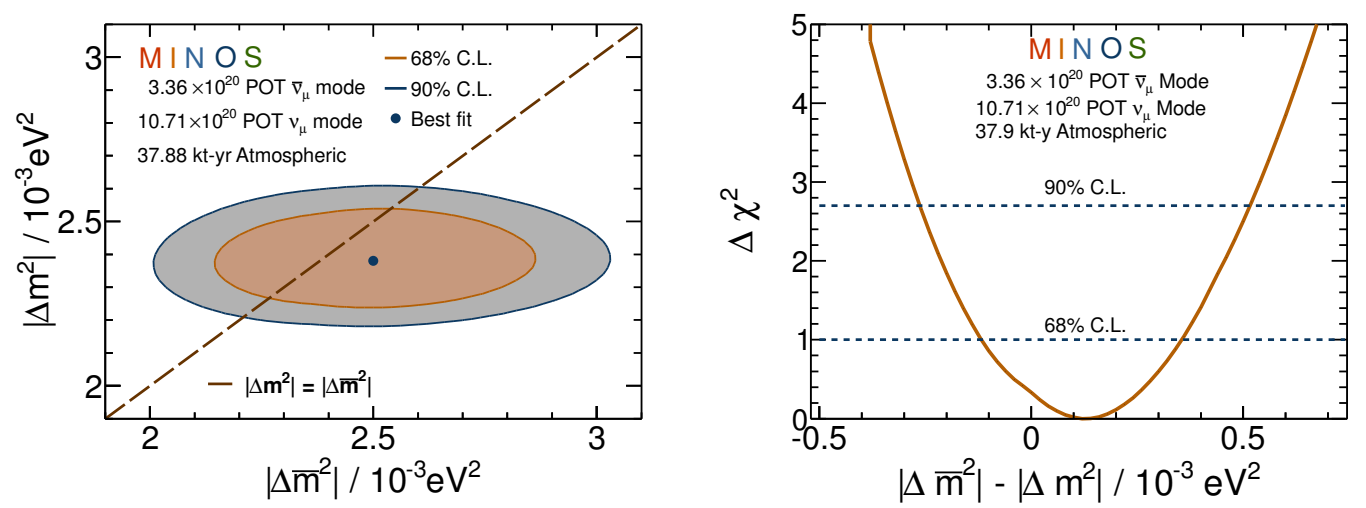

Figure 7.21: Confidence limits (left) obtained for the mass-squared splittings of neutrino $\left(\left|\Delta m^{2}\right|\right)$ and antineutrino $\left(\left|\Delta \bar{m}^{2}\right|\right)$ are projected from fourdimensional log-likelihood surface. The one-dimensional profile of the difference of the mass-squared splittings (right) are marginalized.

Assuming that neutrinos and antineutrinos have identical oscillation parameters, the $\nu_{\mu}$ and $\bar{\nu}_{\mu}$ data sets are combined in one single fit. The bestfit oscillation parameters yield by this fitting, given by:

$$
\begin{aligned}
& \left|\Delta m^{2}\right|=\left[2.40_{-0.11}^{+0.90}\right] \times 10^{-3} \mathrm{eV}^{2}, \\
& \sin ^{2}(2 \theta)=0.955_{-0.035}^{+0.037}(>0.896 \text { at } 90 \% \text { C.L. }) .
\end{aligned}
$$

Figure 7.22 shows the $90 \%$ C.L. allowed region for neutrino oscillation parameters with this assumption. At the maximum mixing angle, $\Delta \chi^{2}=1.59$, which corresponds to the $79.3 \%$ confidence level. 

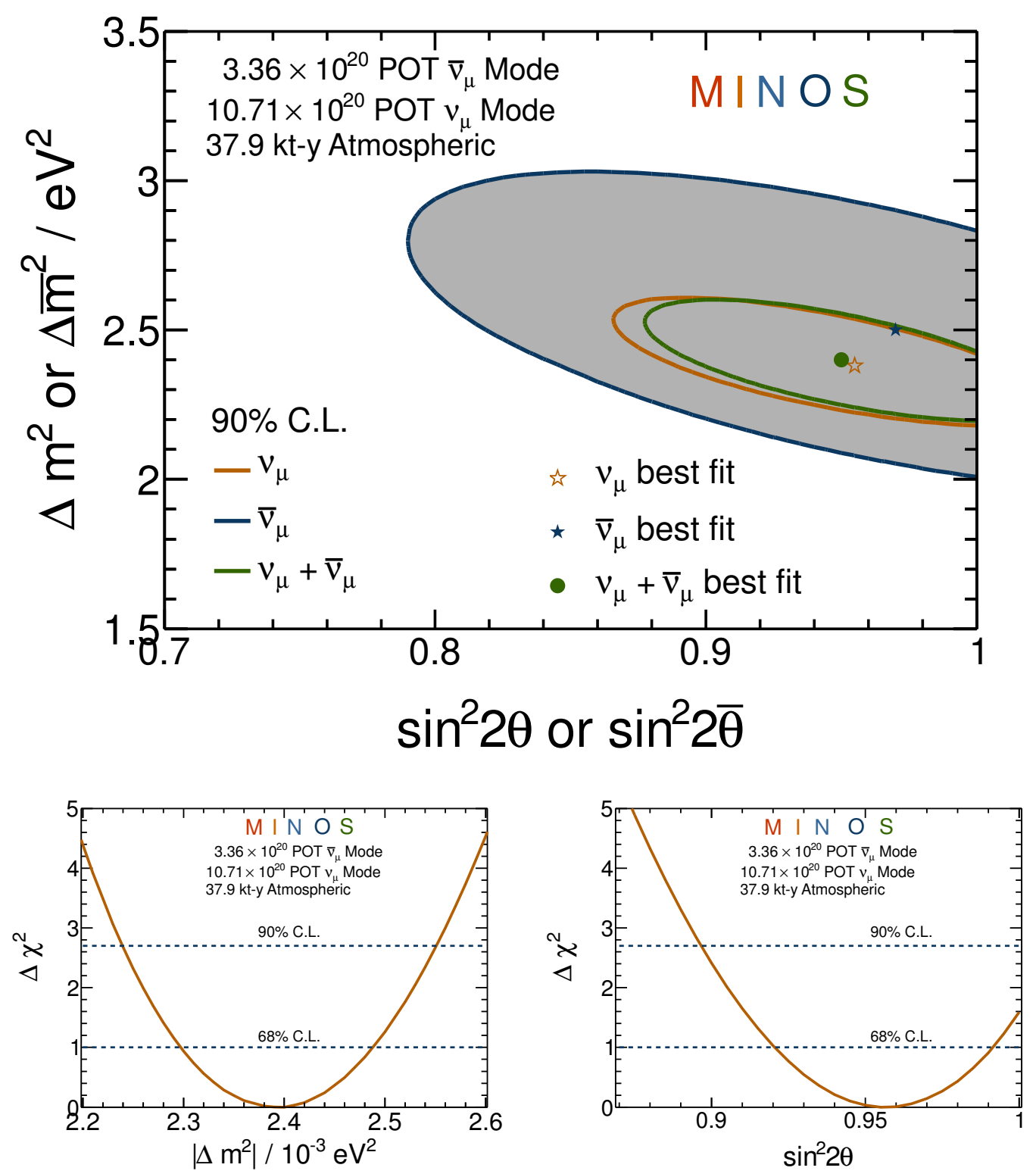

Figure 7.22: The 90\% C.L. contours for neutrino and antineutrino oscillation parameters with either identical or independent oscillation parameter assumption. 


\subsection{Comparison to MINOS results}

The MINOS collaboration has recently published the first ever joint analysis of the accelerator and atmospheric neutrinos in the same experiment [79]. Compared to this publication, my dissertation used an optimized selector for selecting a purer sample of charged-current (CC) $\bar{\nu}_{\mu}$ events in the $\nu_{\mu}$-beam mode. As discussed in Section 4.4.2, this selector increased the signal purity from $71 \%$ to $94 \%$, illustrated in Figure 7.23 . While MINOS reported an observation of $312 \bar{\nu}_{\mu}$-CC events in this beam mode, $216 \bar{\nu}$-CC events were selected by this selector. Figure 7.24 shows the comparison of selected $\bar{\nu}_{\mu}$ data in the $\nu_{\mu}$-beam mode between MINOS publication and this dissertation.

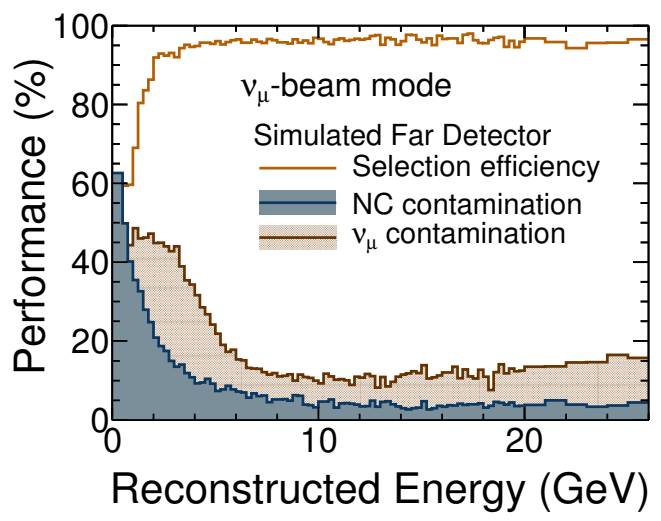

(a) Cut on charge-signed measurement

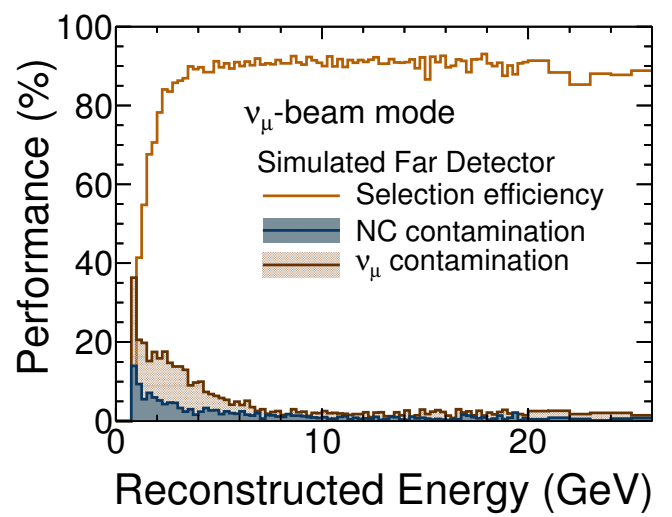

(b) Sequence of cuts

Figure 7.23: The performance of two selectors: cut on charge-signed track measurement (left, used in MINOS published results), and sequence of cuts (right, used in this dissertation). The NC (blue histogram) and wrong-signed (brown histogram) contaminations are reduced significantly with later approach. 


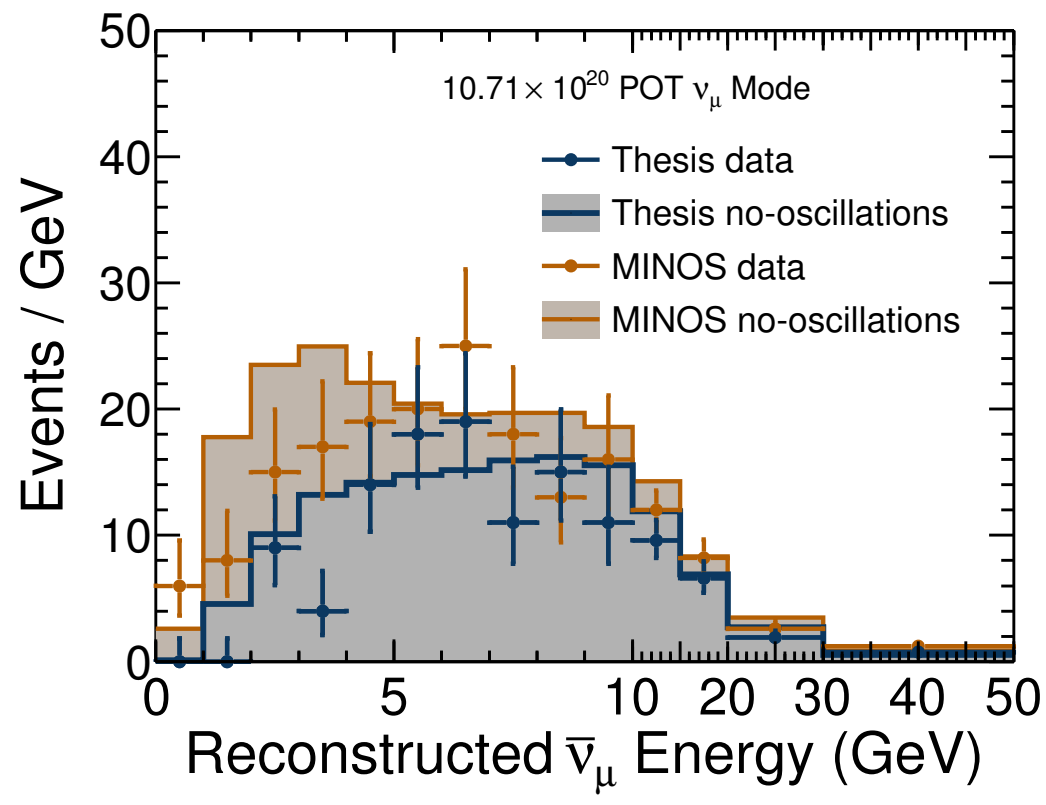

Figure 7.24: Comparison of the selected $\bar{\nu}_{\mu}$-CC sample in the $\nu_{\mu}$-beam mode between MINOS publication and this dissertation. MINOS selected more events but included larger number of neutral-current and wrong-sign background.

However, the $\bar{\nu}$-CC events in the $\nu_{\mu}$-beam mode has an average energy of $5 \mathrm{GeV}$, which is away from the maximum survival probability (1.5 $\mathrm{GeV})$. Also, in the $\bar{\nu}_{\mu}$ disappearance analysis, neutrinos and antineutrinos are extrapolated separately so that the wrong-sign (i.e., $\nu_{\mu}$-CC) background in the selected $\bar{\nu}_{\mu}$ sample is extrapolated as well (mentioned in Section 6.4). If neutrino and antineutrino oscillation are supported by an identical set of oscillation parameters, the measurements with these two data sets should not yield a significant difference in results. 

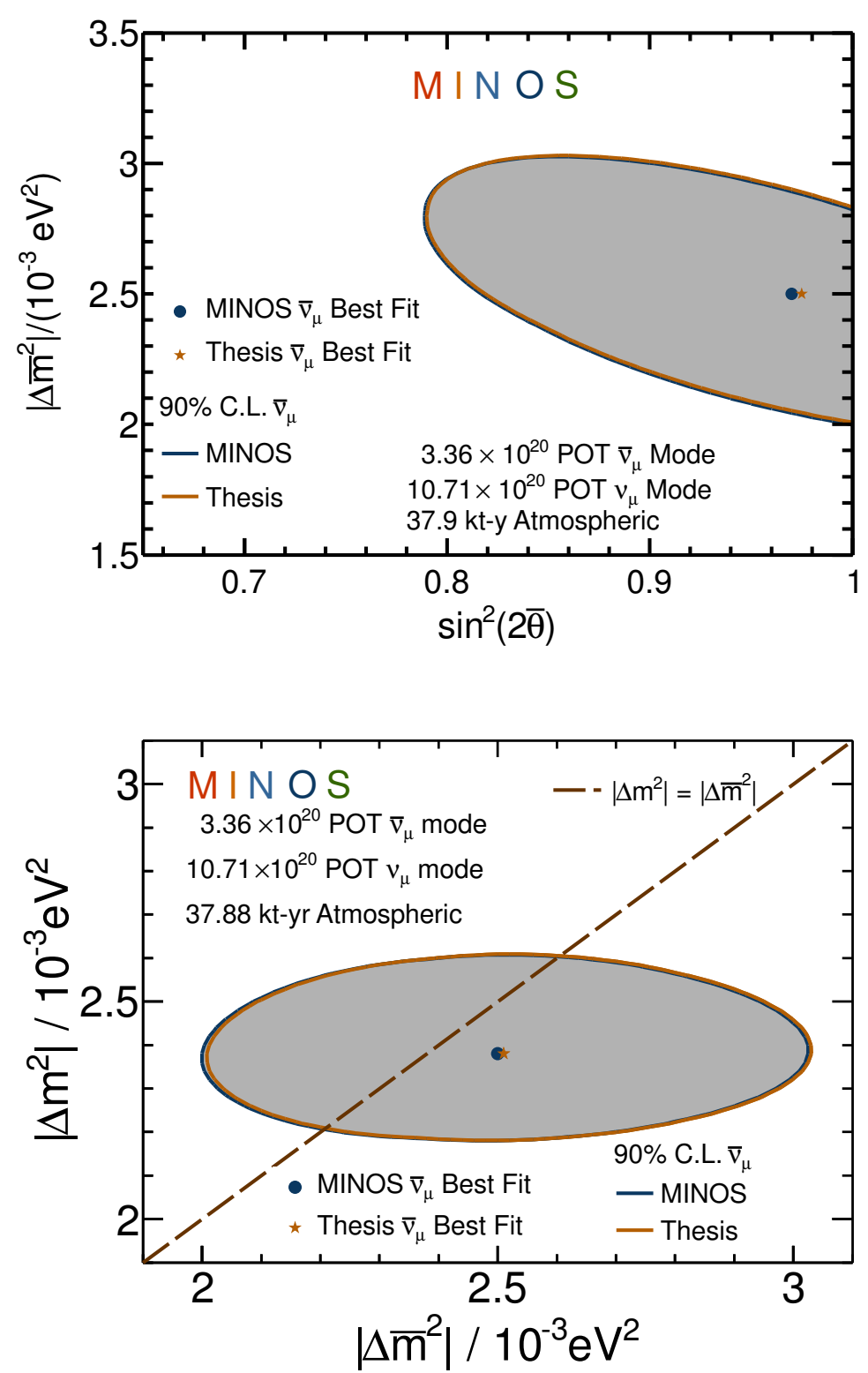

Figure 7.25: The MINOS recently published results are compared to results presented in this dissertation. Four-parameter fitting is used for this comparison. The $90 \%$ C.L. allowed regions of $\bar{\nu}_{\mu}$ oscillation parameters are shown on the left. A marginalization on $\left|\Delta \bar{m}^{2}\right|-\left|\Delta m^{2}\right|$ parameter space are shown on the right. 
MINOS reported the measurement of antineutrino oscillation parameters in four-parameter fit. Figure 7.25 shows the $90 \%$ C.L. contours of antineutrino oscillation parameters and the $\left|\Delta \bar{m}^{2}\right|-\left|\Delta m^{2}\right|$ projection from 4parameter fit. Table 7.1 shows the comparison between the MINOS published results and this dissertation. The results from these two measurement are consistent.

\begin{tabular}{|l|l|l|l|}
\hline results & $\left|\Delta \bar{m}^{2}\right| / 10^{-3} \mathrm{eV}^{2}$ & $\sin ^{2} 2 \bar{\theta}($ at $90 \%$ C.L. $)$ & $\left|\Delta \bar{m}^{2}\right|-\left|\Delta m^{2}\right| / 10^{-3} \mathrm{eV}^{2}$ \\
\hline MINOS & $2.50_{-0.25}^{+0.23}$ & $0.97_{-0.08}^{+0.03}(>0.83)$ & $0.12_{-0.26}^{+0.24}$ \\
\hline Thesis & $2.50_{-0.24}^{+0.24}$ & $0.975_{-0.085}^{+0.025}(>0.835)$ & $0.13_{-0.25}^{+0.23}$ \\
\hline
\end{tabular}

Table 7.1: The MINOS recently published results are compared to results presented in this dissertation.

\subsection{Discussion of dissertation results}

The MINOS experiment observed $\bar{\nu}_{\mu}$-CC events in three independent data sets. The measurements of $\bar{\nu}_{\mu}$-CC disappearance in these data sets were performed either individually or with combined data. The ultimate measurement from the complete MINOS accelerator and atmospheric $\bar{\nu}_{\mu}$-CC data sets was performed in the context of effective two-flavor neutrino oscillation hypothesis. This provided the world's most precise measurement to date of the antineutrino atmospheric mass-squared splitting. Also, a very good agreement between the antineutrino and neutrino mass-squared splitting indicates a consistency with the CPT symmetry. While the impact of systematic un- 
certainties on the antineutrino oscillation parameters was studied, the main uncertainty of antineutrino oscillation parameters comes from the statistical limitation of the data set.

Compared to the latest published results by the MINOS collaboration [79], this dissertation used the optimized selector for selecting purely charged-current $\bar{\nu}_{\mu}$ events in the $\nu_{\mu}$-beam mode. Since this type of event has a fairly small sensitivity to the antineutrino oscillation parameters, the difference between these two measurements is small.

The disappearance of $\bar{\nu}_{\mu}$ events was well-described in effective two-flavor neutrino framework. By considering the matter effect on the atmospheric neutrinos and antineutrinos $\left(L / E_{\nu} \sim 10^{4} \mathrm{~km} / \mathrm{GeV}\right)$ and small appearance of $\nu_{e}$, MINOS performed an additional analysis with the exact three-flavor model [51]. This analysis shows a very limited sensitivity to the neutrino mass hierarchy and the CP-violating phase. Thus, we decided to describe the MINOS $\nu_{\mu}$ and $\bar{\nu}_{\mu}$ disappearance data only with an effective two-flavor neutrino model in the context of this dissertation. 


\section{Chapter 8}

\section{Summary and outlook}

\subsection{Summary of this dissertation}

This dissertation presented the complete measurements of antineutrino oscillation parameters in the MINOS detector. The new hadronic shower energy estimator was implemented to improve the sensitivity to the antineutrino oscillation parameters. A suggestion for improving the charged-current $\bar{\nu}_{\mu}$ and neutral-current identification was presented. Compared to the latest published measurement by the MINOS collaboration [79], the selection for $\bar{\nu}_{\mu}$ in the $\nu_{\mu}$ beam was updated to get purer sample. The selection for $\bar{\nu}_{\mu}$ in the $\bar{\nu}_{\mu}$ beam remained unchanged. The author of this dissertation conducted the measurements of antineutrino oscillation parameters with both the individual $\bar{\nu}_{\mu}$ data set and with the combined data. A total of 710 candidate $\bar{\nu}_{\mu}$ charged-current events was observed. Compared to 887 events with the no-oscillation prediction, the $\bar{\nu}_{\mu}$ deficit was well described by the two effective two-flavor neutrino model, given by:

$$
\begin{aligned}
& \left|\Delta \bar{m}^{2}\right|=\left[2.50_{-0.29}^{+0.23}\right] \times 10^{-3} \mathrm{eV}^{2}, \\
& \sin ^{2}(2 \bar{\theta})=0.97_{-0.08}^{+0.03}(>0.83 \text { at } 90 \% \text { C.L. }) .
\end{aligned}
$$


To test the CPT symmetry in the lepton sector, a fit for $\bar{\nu}_{\mu}$ and $\nu_{\mu}$ disappearance in MINOS was performed simultaneously with the assumption that neutrino and antineutrino oscillations are supported by the independent sets of oscillation parameters. This fit yielded a consistency between the neutrino and antineutrino mass-squared splittings by:

$$
\left|\Delta \bar{m}^{2}\right|-\left|\Delta m^{2}\right|=0.13_{-0.25}^{+0.23} \times 10^{-3} e V^{2} .
$$

The uncertainty of this measurement was primarily driven by the statistical uncertainty in the $\bar{\nu}_{\mu}$ data set.

Assuming that neutrino and antineutrino oscillations were supported by the identical set of oscillation parameters, a measurement with combined $\bar{\nu}_{\mu}$ and $\nu_{\mu}$ disappearances was performed. This disfavors the maximum mixing angle at the $79.3 \%$ confidence level.

\subsection{Outlook on the antineutrino oscillations measure- ments}

The uncertainty of the measurement of antineutrino oscillation parameters from the MINOS charged-current $\bar{\nu}_{\mu}$ disappearance is driven by the statistical uncertainty. In the era of the MINOS+ experiment, we have an opportunity to update this measurement with a substantial increase of $\bar{\nu}_{\mu}$ events. After finishing three years of operation under the MINOS+ experiment, we expect to collect a total of 65 kton-years atmospheric neutrinos and antineutrinos, i.e., a $70 \%$ statistical increase. Also, if the $\bar{\nu}_{\mu}$-beam mode from the NuMI is delivered to the NOvA Far Detector, additional $\bar{\nu}_{\mu}$ events will be observed 
in the MINOS detector. Thus, MINOS+ can measure antineutrino oscillations and test the CPT invariant with a higher precision than the results reported in this dissertation.

Because of the hadron production of proton-nucleus interactions and a lower charged-current $\bar{\nu}_{\mu}$ cross-sections, the probability of $\bar{\nu}_{\mu}$ event observation per protons on target is about one third compared to $\nu_{\mu}$ observations. Thus, running neutrino experiments in the $\bar{\nu}_{\mu}$ beam mode is not a priority when neutrino statistics is the most ultimate goal. The T2K experiment is exploring neutrino oscillation physics with a $\nu_{\mu}$ beam. The NOvA experiment also plans to run with the $\nu_{\mu}$ beam at the beginning of its operation. However, both will potentially switch into the $\bar{\nu}_{\mu}$ beam in order to study the neutrino mass hierarchy and the CP-violating phase. If that is the case, it enables one to update our understanding of antineutrino oscillations in both $\bar{\nu}_{\mu}$ disappearance and $\bar{\nu}_{\mu} \rightarrow \bar{\nu}_{e}$ appearance channels.

In the next few decades, exploring neutrino physics with the $\bar{\nu}_{\mu} \rightarrow \bar{\nu}_{e}$ appearance channel will be essential since this channel is sensitive to the CPviolating phase. Also, the matter treats neutrinos and antineutrinos in a different manner. This gives us an opportunity to resolve the mass hierarchy and look for CP violation in the lepton sector. Precision measurement of $\bar{\nu}_{\mu} \rightarrow \bar{\nu}_{e}$ transitions in comparison to its $\nu_{\mu} \rightarrow \nu_{e}$ transitions would be an important guideline for the long baseline neutrino experiments in order to understand the nature of this elusive particle. 


\subsection{Conclusion}

The measurements presented in this dissertation have improved the world's understanding of antineutrino oscillations from a direct observation of $\bar{\nu}_{\mu}$ disappearance. The results provided the world's most precise measurement of antineutrino mass-squared splitting in the atmospheric sector. In consideration that neutrino and antineutrino oscillations are driven by the different sets of parameters, a CPT examination was performed and yielded a consistency between neutrino and antineutrino oscillation parameters.

This dissertation reported the very first joint analysis of accelerator and atmospheric neutrinos in the same experiment. In the next few decades, the joint analysis with combined data sets from different channels and from different experiment should be considered further. This will provide a powerful tool for solving the degeneracy and correlation among neutrino oscillation parameters and reaching definite answers for the nature of neutrinos.

Neutrinos, since their on-paper appearance in the 1930s, have proved to be one of the most interesting elementary particles. The massiveness of neutrinos, which is implied by the well-grounded neutrino oscillation phenomena, is beyond the reach of the Standard Model. These elusive particles may have even more "anomalous" properties than what we have already seen. The potential of uncovering presents exciting avenues for the growing field of neutrino physics. 
Appendices 


\section{Appendix A}

\section{Degeneracy and correlation among oscillation parameters}

The T2K and NOvA experiments will explore neutrino physics from the $\vec{\nu}_{\mu} \rightarrow \vec{\nu}_{e}$ channels since these channels facilitate the measurement of mixing angle $\Delta_{13}$ and $\mathrm{CP}$-violating phase $\delta_{\mathrm{CP}}$. For the second order expansion in $\sin \theta_{13}$ and $\alpha \equiv \frac{\Delta m_{21}^{2}}{\Delta m_{31}^{2}}$, the $\nu_{e}$ appearance probability is given by [54]:

$$
\begin{aligned}
P_{\nu_{\mu} \rightarrow \nu_{e}}= & \alpha^{2} \sin ^{2} 2 \theta_{12} c_{23}^{2} \frac{\sin ^{2} A \Delta}{A^{2}}+4 s_{13}^{2} s_{23}^{2} \frac{\sin ^{2}(A-1) \Delta}{(A-1)^{2}} \\
& +2 \alpha s_{13} \sin 2 \theta_{12} \sin 2 \theta_{23} \cos \left(\Delta+\delta_{\mathrm{CP}}\right) \frac{\sin A \Delta \sin (A-1) \Delta}{A} \frac{\Delta-1}{A-1}
\end{aligned}
$$

where

$$
\Delta \equiv \frac{\Delta m_{31}^{2} L}{4 E}, \quad A \equiv \frac{V L}{2 \Delta}
$$

A correlation exists between $\theta_{13}$ and $\delta_{\mathrm{CP}}$. Assuming that $\left(\theta_{13}^{*}, \delta_{\mathrm{CP}}^{*}\right)$ are the true values. The $\left(\theta_{13}, \delta_{\mathrm{CP}}\right)$ solutions are given by:

$$
P_{\nu_{\mu} \rightarrow \nu_{e}}\left(\theta_{13}, \delta\right)=P_{\nu_{\mu} \rightarrow \nu_{e}}\left(\theta_{13}^{*}, \delta_{\mathrm{CP}}^{*}\right)
$$

and are not unique but continuously degenerate. Figure A.1 shows the equiprobability curve of $P\left(\nu_{\mu} \rightarrow \nu_{e}\right)$ on the $\left(\theta_{13}, \delta_{\mathrm{CP}}\right)$ surface. 

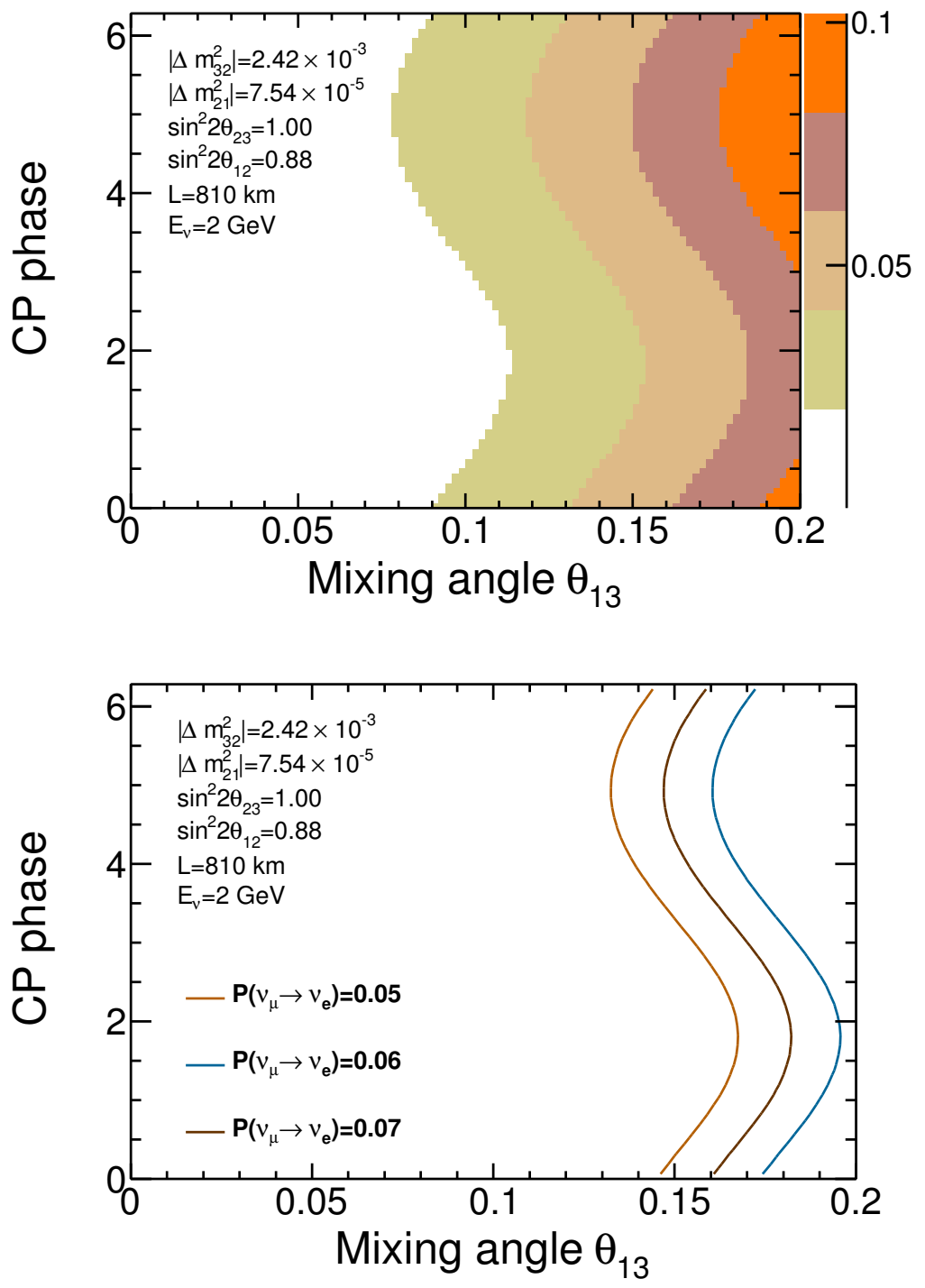

Figure A.1: Equiprobability curves of $P\left(\nu_{\mu} \rightarrow \nu_{e}\right)$. 
Unfortunately, introducing $P_{\bar{\nu}_{\mu} \rightarrow \bar{\nu}_{e}}\left(\theta_{13}, \delta\right)$ can not completely solve the problem. Figure A.2a shows a clone solution, called an intrinsic clone solution, given by:

$$
P_{\nu_{\mu} \rightarrow \nu_{e}}^{ \pm}\left(\theta_{13}, \delta_{\mathrm{CP}}\right)=P_{\nu_{\mu} \rightarrow \nu_{e}}^{ \pm}\left(\theta_{13}^{*}, \delta_{\mathrm{CP}}^{*}\right)
$$

where $+(-)$ is for neutrino (antineutrino). This issue can be solved by adding information from a different channel or using a different baseline. Figure A.2b shows the equiprobability curves with different baselines, which can be used to solve the intrinsic clone introduced by measuring of $\bar{\nu}_{e}$ appearance.

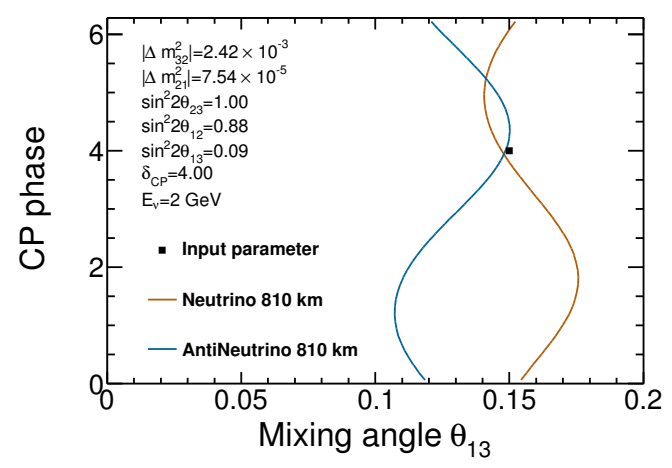

(a)

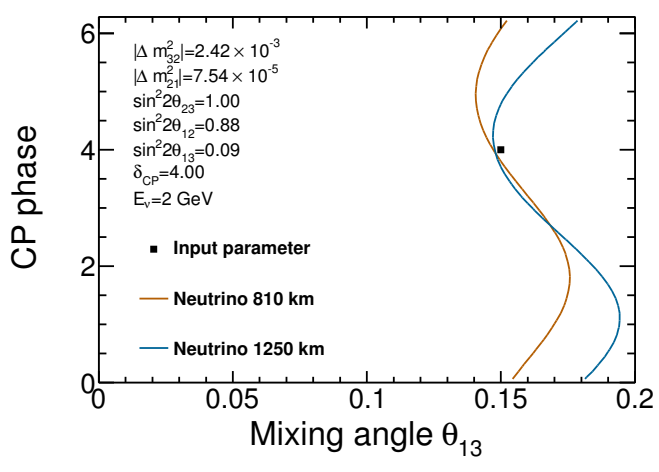

(b)

Figure A.2: Intrinsic clone due when introducing additional measurements with either $\bar{\nu}_{\mu} \rightarrow \bar{\nu}_{e}$ appearance (left) or different baseline (right).

However, this intrinsic degeneracy is only part of the "clone solution problem". Two other sources of ambiguity are:

- The sign of $\Delta m_{32}^{2}$ or the mass hierarchy. We use the parameter $s_{\text {atm }}$ to describe this. We assign $s_{\text {atm }}=1$ for a normal hierarchy and $s_{\text {atm }}=$ 
-1 for a inverted hierarchy. Figure A.3a illustrates the clone solution introduced by two different mass hierarchy.

- The octant of $\theta_{32}$. We use the parameter $s_{\operatorname{th} 23}$ to describe this. $s_{\operatorname{th} 23}=1$ for $\theta_{23}<45^{\circ}$ and $s_{\text {th23 }}=-1$ for $\theta_{23}>45^{\circ}$. Figure A.3 depicts the clone solutions introduced by any combination of mass hierarchy and octant of $\theta_{23}$.

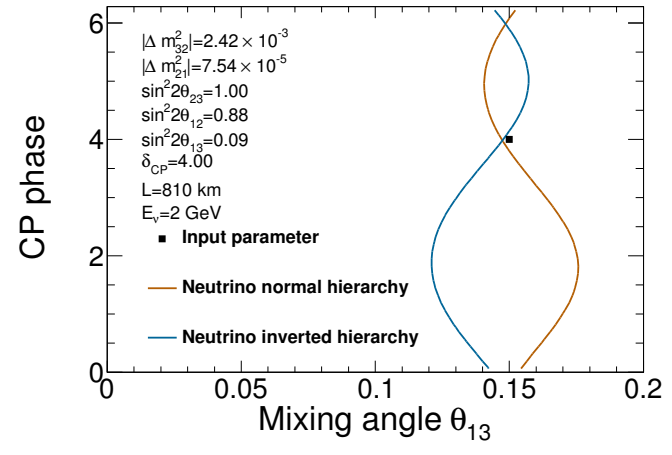

(a)

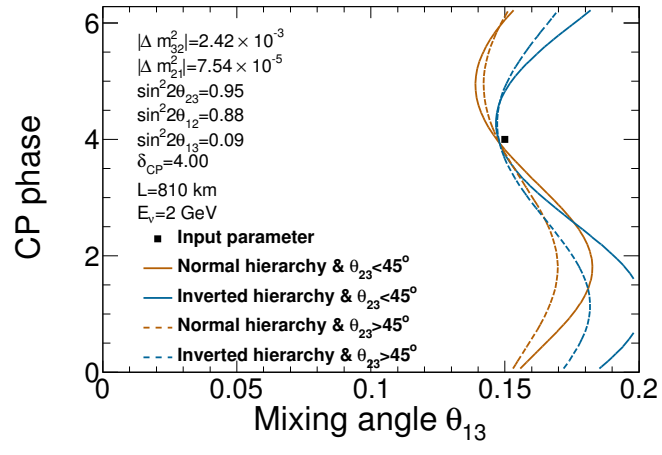

(b)

Figure A.3: Degeneracy of mass hierarchy (left) and $\theta_{23}$-octant (right) in $\bar{\nu}_{\mu} \rightarrow$ $\bar{\nu}_{e}$ appearance channel.

Summing these ambiguities, we have a system of equations:

$$
P_{\nu_{\mu} \rightarrow \nu_{e}}^{ \pm}\left(\theta_{13}, \delta_{\mathrm{CP}}, s_{\mathrm{atm}}, s_{\mathrm{th} 23}\right)=P_{\nu_{\mu} \rightarrow \nu_{e}}^{ \pm}\left(\theta_{13}^{*}, \delta_{\mathrm{CP}}^{*}, s_{\mathrm{atm}}^{*}, s_{\mathrm{th} 23}^{*}\right)
$$

Solving these equations leads to a true solution plus additional clone solutions which form an eightfold degeneracy [141], as illustrated in Figure A.4. 


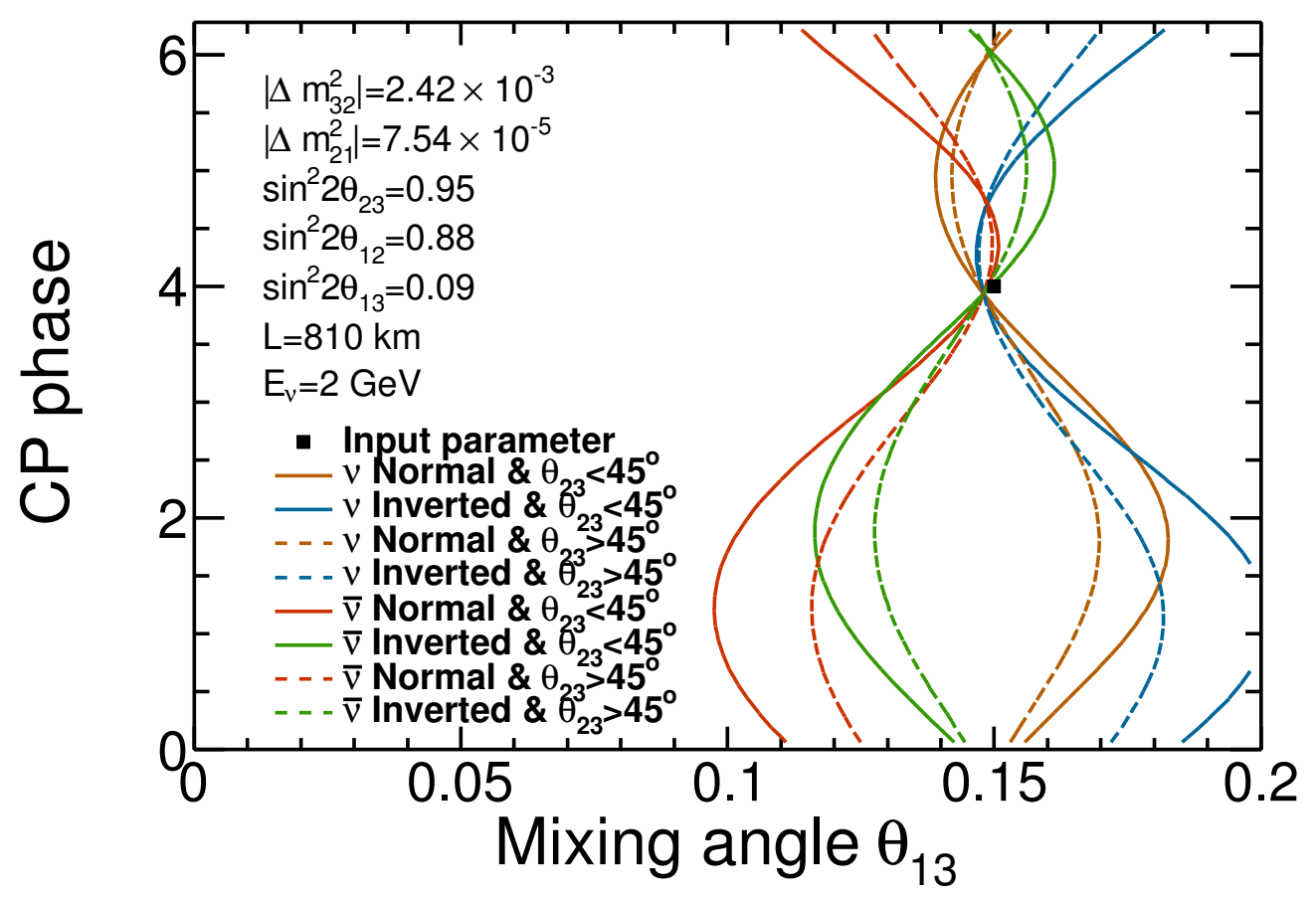

Figure A.4: Eightfold degeneracy in $\bar{\nu}_{\mu} \rightarrow \bar{\nu}_{e}$ appearance channel. 


\section{Appendix B}

\section{The SKZP reweighting effect on oscillation parameters}

Figure B.1 shows the effect of SKZP reweighting on the Far Detector prediction. The SKZP reweighting mainly affects the falling edge of energy spectrum $(>4 \mathrm{GeV})$. Since the oscillation dip is around $1.5 \mathrm{GeV}$, the effect of SKZP reweighting is not sensitive to the measurement of oscillation parameters.
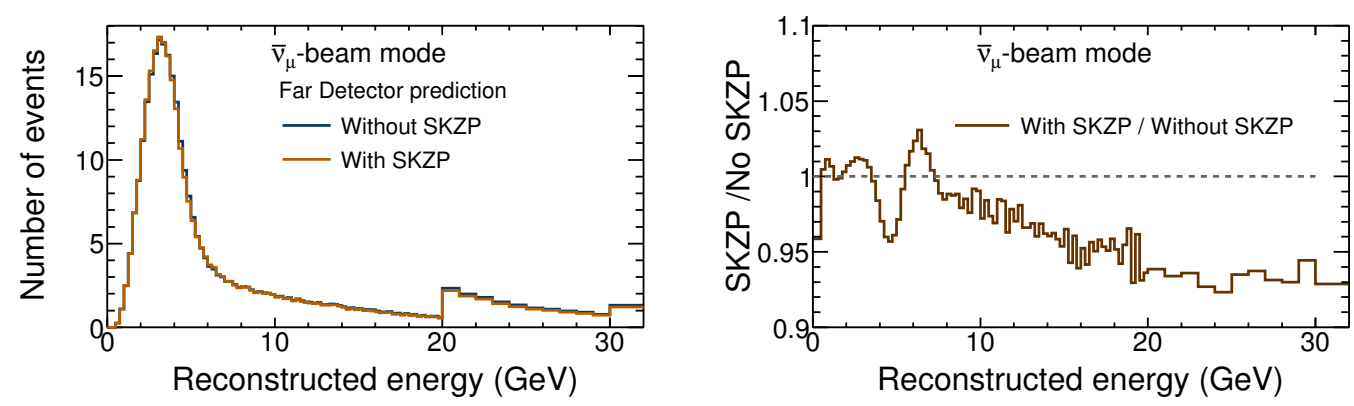

Figure B.1: Comparison of Far Detector prediction with (orange) and without (blue) SKZP reweighting. The energy spectra are shown on the left and their ratio is shown on the right.

The measurements of oscillation parameters $\Delta \bar{m}^{2} / \sin ^{2} 2 \bar{\theta}$ with and without SKZP, yielded $2.64 \times 10^{-3} \mathrm{eV}^{2} / 0.95$ and $2.65 \times 10^{-3} \mathrm{eV}^{2} / 0.95$ respectively.

Figure B.2 shows the SKZP reweighting effect on confidence contour and its 
projections separately on $\Delta \bar{m}^{2}$ and $\sin ^{2} 2 \bar{\theta}$. Apparently, the effect is much smaller than the uncertainties of the oscillation parameters.

In summary, the SKZP reweighting enforces the agreement between the Monte Carlo simulation and the Near Detector data but does not change antineutrino oscillation parameters which are measured by the $\bar{\nu}_{\mu}$ disappearance analysis.
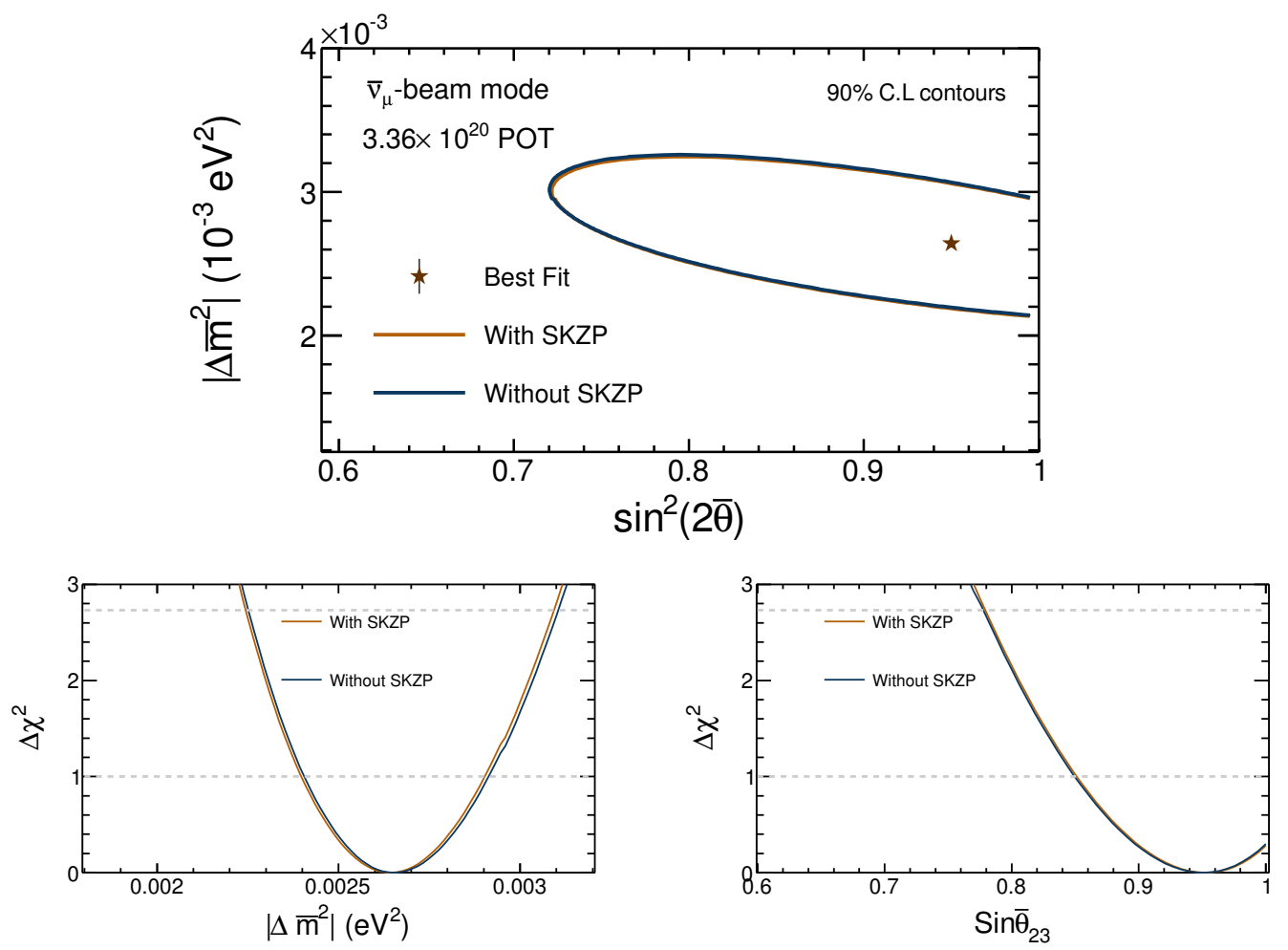

Figure B.2: The confidence contours (top) with (orange) and without (blue) applying SKZP weighting. Their projections on mixing angle, $\sin ^{2}(2 \bar{\theta})$ and on mass-squared splitting, $\left|\Delta \bar{m}^{2}\right|$ are shown on the bottom left and on the bottom right respectively. 


\section{Appendix C}

\section{Fitting in bins of energy resolution}

To improve the sensitivity to the measurement of neutrino oscillation parameters, the fitting in bins of either estimated energy resolution or $L[k m] / E[G e V]$ resolution was adopted. The former is implemented for the $\nu_{\mu}$ disappearance analysis from $\nu_{\mu}$-beam mode [58], and the latter is applied for the atmospheric $\nu_{\mu}$ and $\bar{\nu}_{\mu}$ disappearance analysis [127]. However, this technique was not adopted for the accelerator $\bar{\nu}_{\mu}$ disappearance analysis because of the statistic limitation. Figure C.1a shows the fractional resolution $\sigma_{E} / E$ as the function of reconstructed energy for selected $\bar{\nu}_{\mu}$-CC events in $\bar{\nu}_{\mu}$-beam mode.

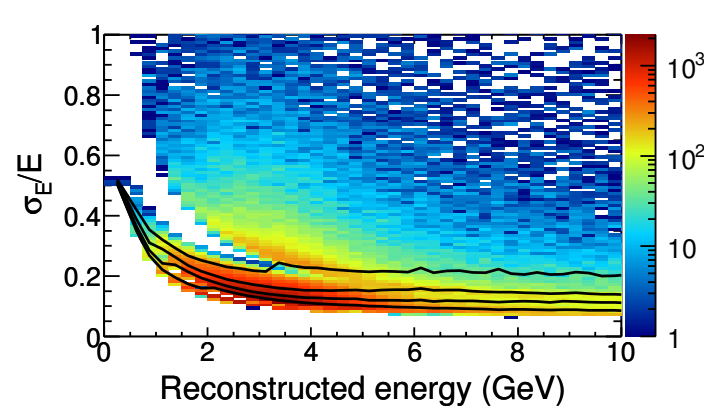

(a)

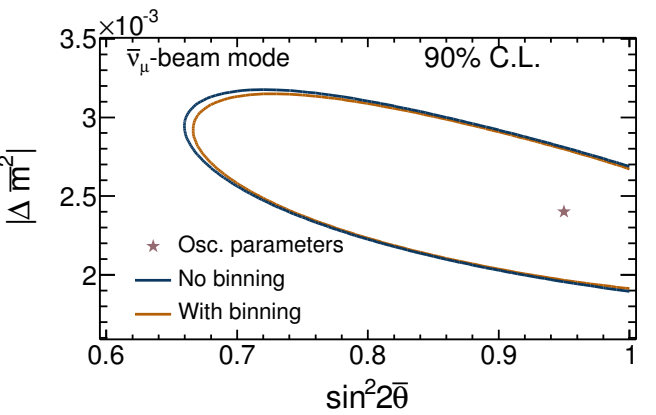

(b)

Figure C.1: The fractional resolution $\sigma_{E} / E$ as the function of reconstructed energy for selected $\bar{\nu}_{\mu}$-CC events in $\bar{\nu}_{\mu}$-beam mode (left) and the sensitivity gain with fitting in five bins of energy resolution. 
The selected sample is divided into five sub-samples which are fitted independently. Figure C.1b shows the sensitivity contour by adding statistically contours from fitting these sub-samples. The improvement is so small that we did not apply it in this dissertation.

The author of this dissertation has adopted this technique for the measurement of $\nu_{\mu}$-CC disappearance analysis in the MINOS+ experiment. The selected $\nu_{\mu}$-CC sample is divided into five sub-samples based on the event estimated fractional resolution. Figure C.2 shows the ratio of the no-oscillation prediction to the oscillated prediction for five sub-samples and their corresponding $90 \%$ C.L. regions. The smaller labeled bin corresponds to the better resolution sample. The sharper oscillation dip and smaller sensitivity contour with smaller labeled bin confirms that we correctly parameterize the energy resolution.
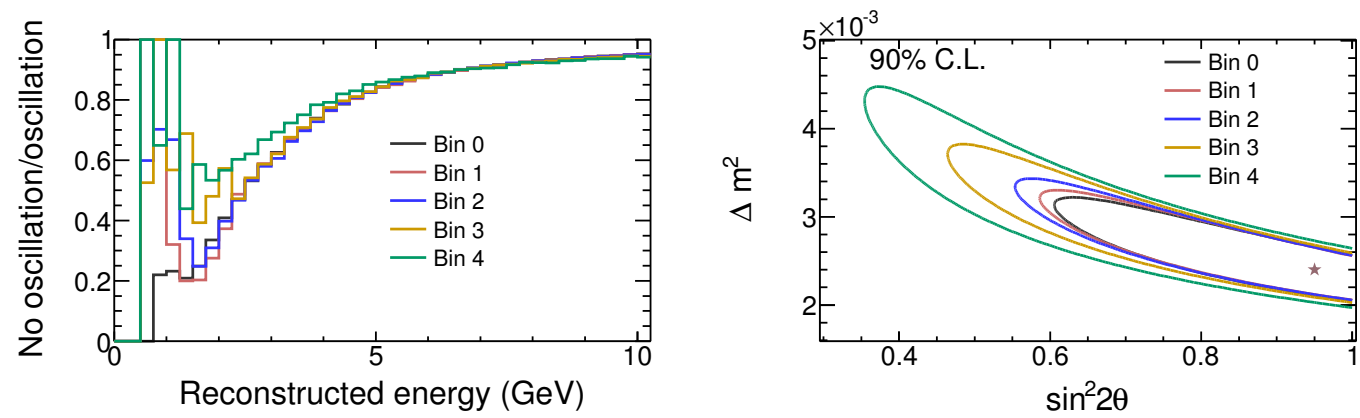

Figure C.2: The ratio of null-oscillation prediction to the oscillated prediction for five sub-samples is shown on the left and their corresponding 90\% C.L. regions are shown on the right. The data sample is scaled to $18 \times 10^{20} \mathrm{POT}$ exposure (expected in three years of the MINOS+ operation.)

Figure C.3 shows the projection of sensitivity contours on the two oscillation 
parameters. Apparently, the better energy resolution of the sub-sample, the more precise the constraint on oscillation parameters becomes.
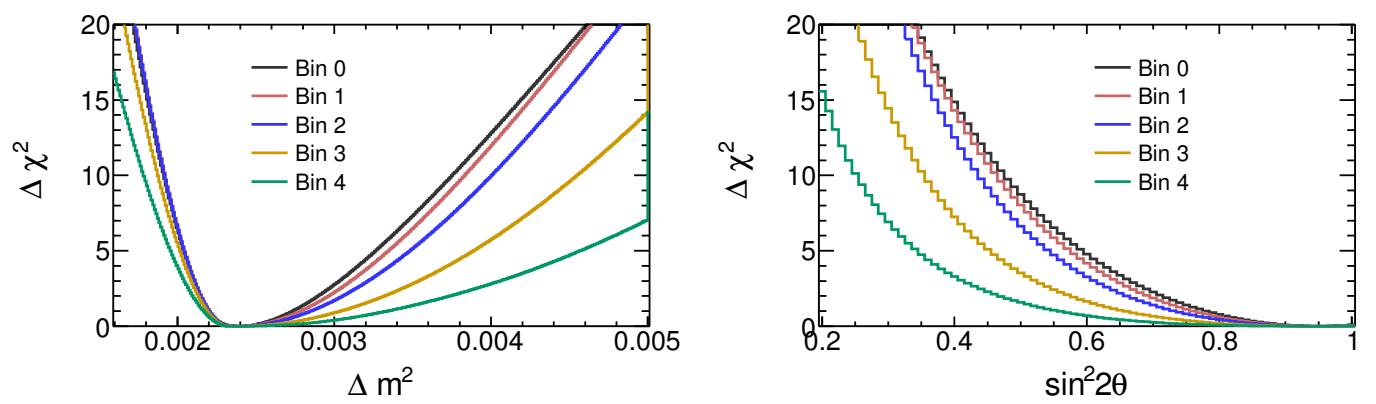

Figure C.3: The projections of sensitivity contours on the $\Delta m^{2}$ (left) and $\sin ^{2} 2 \theta$ (right) axis.

Figure C.4 shows the sensitivity gain by applying the fitting in bins of energy resolution in comparison to the default fitting. The improvement matches to a $10 \%$ statistical increase of the current data.

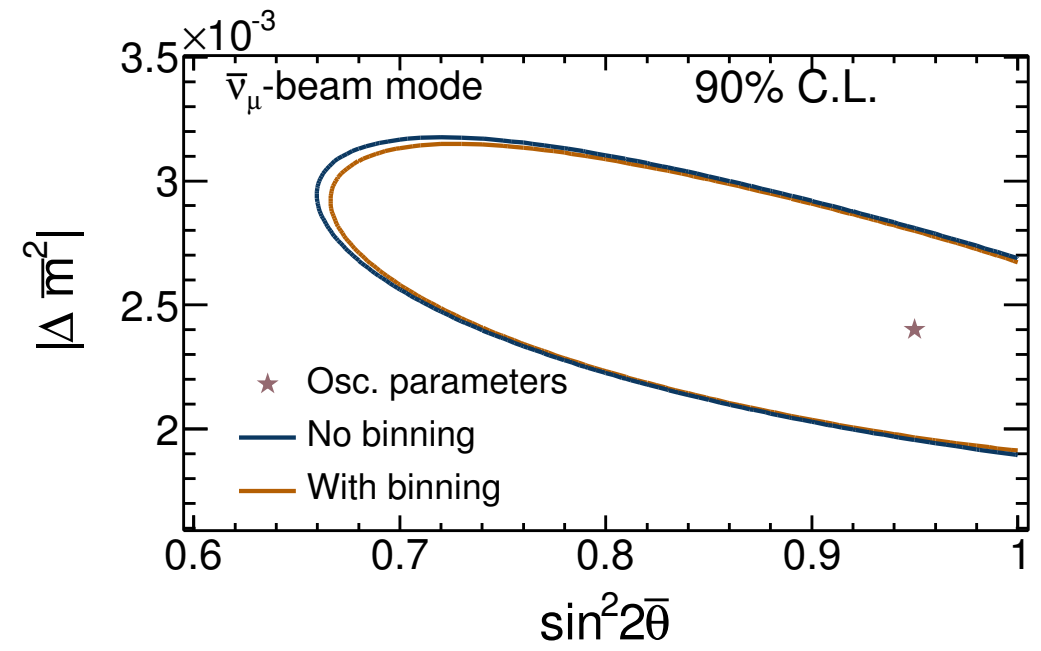

Figure C.4: The sensitivity gain by performing the fitting in bins of energy resolution. The data sample is scaled to $18 \times 10^{20}$ POT exposure (expected in three years of the MINOS+ operation.) 


\section{Appendix D}

\section{Mass hierarchy resolvability with long-baseline experiments}

To solve the mass hierarchy, both $\nu_{\mu}$ and $\bar{\nu}_{\mu}$ beams will be used. The neutrino and antineutrino bi-probability with different baselines, NOvA (810 $\mathrm{km})$ and LBNE $(1250 \mathrm{~km})$ for different hierarchies, assumes the maximal $\theta_{23}$ are showed in Figure D.1. In other words, the capability of resolving the mass hierarchy in long-baseline experiments depends on the octant of the mixing angle $\theta_{23}$.
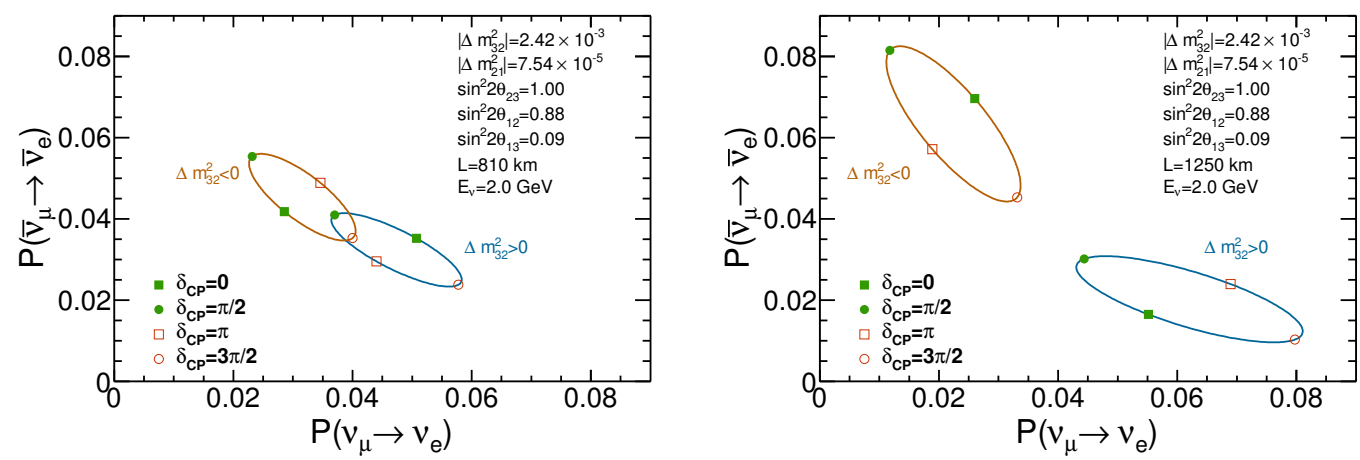

Figure D.1: The bi-probability with different baseline, NOvA $(810 \mathrm{~km})$ and LBNE $(1250 \mathrm{~km})$ for different hierarchy, assuming the maximal $\theta_{23}$.

Evidently, the longer baseline with the LBNE proposal shows better mass hierarchy resolvability. When the octant of $\theta_{23}$ are taken into account, as 
depicted in Figure D.2, the mass hierarchy can be solved better with NOvA baseline when the $\theta_{23}>45^{\circ}$

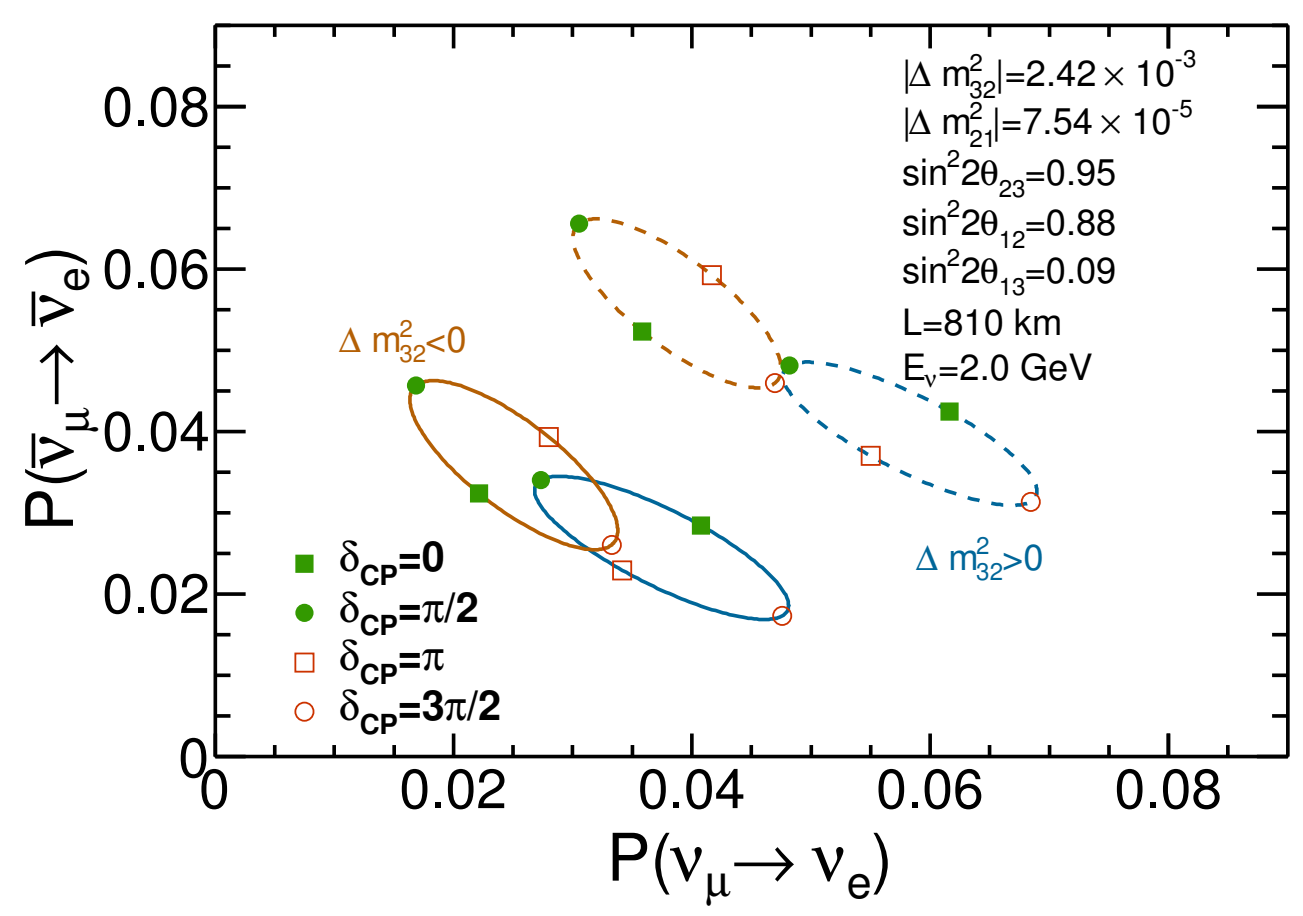

Figure D.2: The bi-probability with baseline of NOvA $(810 \mathrm{~km})$ for different mass hierarchy and octant of $\theta_{23}$. The lower octant is showed in solid curves while the higher octant is in dashed curves. 


\section{Appendix E}

\section{Auxiliary Detector and measurement of neutrino velocity in MINOS}

This chapter introduces the motivations and describes the setup of the Auxiliary Detectors (AD) for measuring the neutrino velocity in the MINOS experiment. These small detectors are paired with and simultaneously timestamp the neutrino events in the two MINOS detectors. This setup enables one to measure precisely the electronic latencies of the MINOS detectors and relative latency between these two large detectors.

\section{E.1 Motivations}

\section{Measuring neutrino velocity}

From cosmological constraints and from neutrinoless double beta decays, neutrinos widely have been known to have a very tiny mass (on the order of few eV). The relativistic velocity of $3 \mathrm{GeV}$ neutrinos (assume mass $\approx$ $\left.3 \mathrm{eV} / c^{2}\right)$ should satisfy:

$$
\gamma=\frac{p}{m_{0} c} \approx \frac{E}{m_{0} c}=10^{9}
$$

where $\gamma$ is the Lorentz factor, $m_{o}, p$ and $E$ are respectively the mass, the momentum and the energy of neutrinos, and $c$ is the speed of light. Considering 
the definition of Lorentz factor, the difference between the neutrino and light speeds is at the level of $\frac{|v-c|}{c}<10^{-18}$. However, due to some hypothetical Lorentz violation effect [142], which changes the energy-momentum dispersion relationship, neutrinos with energies of a few $\mathrm{GeV}$ travel with velocities $\frac{|v-c|}{c} \approx$ $10^{-4}$ different than the speed of light.

The measurement of neutrino velocity is conceptually straightforward. All we need is the distance between the two detectors and the time it takes for a neutrino to propagate between them. The MINOS experiment with two detectors separated by the $734,286.8 \pm 0.5 \mathrm{~m}$ baseline, has made previous measurements. The initial results [143], published in 2007 with one year of collecting data, indicated that neutrinos arrived at the Far Detector slightly earlier than expected:

$$
\delta t=t_{\nu}-t_{c}=126 \pm 32 \text { (stat.) } \pm 64 \text { (syst.), }
$$

where $t_{\nu}$ and $t_{c}$ are respectively the propagation time interval of neutrinos and of photons in vacuum. This result was converted to a neutrino velocity of $v / c-1=(5.1 \pm 2.9) \times 10^{-5}$, which was consistent with the speed of light to less than $1.8 \sigma$.

In September 2011, the OPERA experiment claimed an observation of superluminal neutrinos from the data they collected at the Gran Sasso laboratory, $730 \mathrm{~km}$ downstream from CERN. However, this observation was subsequently found to be wrong and OPERA later reported their corrected result of neutrino velocity, which is consistent with the speed of light. In response to this claim, the MINOS experiment updated their timing system 
with a number of studies, combined with a statistical increase by a factor of 8.5 in collected neutrino data, to revisit the measurement of neutrino velocity with a more accurate result.

\section{Introducing Auxiliary Detector (AD)}

The signals of neutrino interactions, recorded by the deposited energy hits in the detectors, have to pass through complicated detector electronics before they are time-stamped by the readout system. These electronics introduce a some amount of latency between the moment neutrinos hit the detectors and the time they are recorded. In principle, this delay could be obtained by measuring the path length of each electronic component. However, this approach leads to a large uncertainty. Instead, the measurement of latency of the whole detectors and electronic system in situ was considered.

The two MINOS detectors use different readout systems. The introduced AD, serves as the mediate electronic system, helps to connect electronic systems between the Near Detector (ND) and Far Detector (FD). The rela-

tive electronic latency of the AD and corresponding MINOS detector is firstly obtained by matching muons observed by both detectors. Then, the relative ND-FD electronic latency is derived by subtracting the relative latency between the two sites.

Two identical, small portable Auxiliary Detectors (ADs), essentially muon counters, were installed for this purpose. Figure E.1 shows the schematic design and installation positions of ADs at the two sites of MINOS detectors. 


\section{E.2 Auxiliary Detector and readout system}

Each AD is made of two $63 \mathrm{~cm}$ x $57 \mathrm{~cm}$ "MINOS-style" plastic scintillator planes, which comprise of 16 scintillator strips, read out by wavelengthshifting fibers and 16-anode PMTs. Each PMT is shielded with low magnetic permeable material to mitigate effects of the magnetic field at both MINOS detectors. Due to physics reasons and easy access considerations, one AD was placed at the backend of the ND, and a sustainable location (from the magnetic field's strength point of view) at the FD site was chosen to be in the gap between the two super-modules. Two readout systems, used to time-stamp the arrival of muons at the ADs, are shortly described below:

\section{CAMAC TDC}

The TDC 4208 is used to measure the time interval between the dynode coincidence signals and the trigger signals at the MINOS detectors ${ }^{1}$. The TDC internal clock is $125 \mathrm{MHz}$ and the resolution is $1 \mathrm{~ns}$. The TDC limitation to a dynamic range of $8.3 \mathrm{~ms}$ only permits us to use this readout system at the ND site since the time information of the spill arrival is well-known. At the FD site, the stochastic arrival of cosmic muons ${ }^{2}$ requires us to have a different readout with a larger dynamic range.

\footnotetext{
${ }^{1}$ To mitigate the systematics, the AD and MINOS detector at each site triggers with the same signal.

${ }^{2}$ The $\mathrm{AD}$ at FD site records the cosmic muons since the spill muon rate at FD is so small.
} 


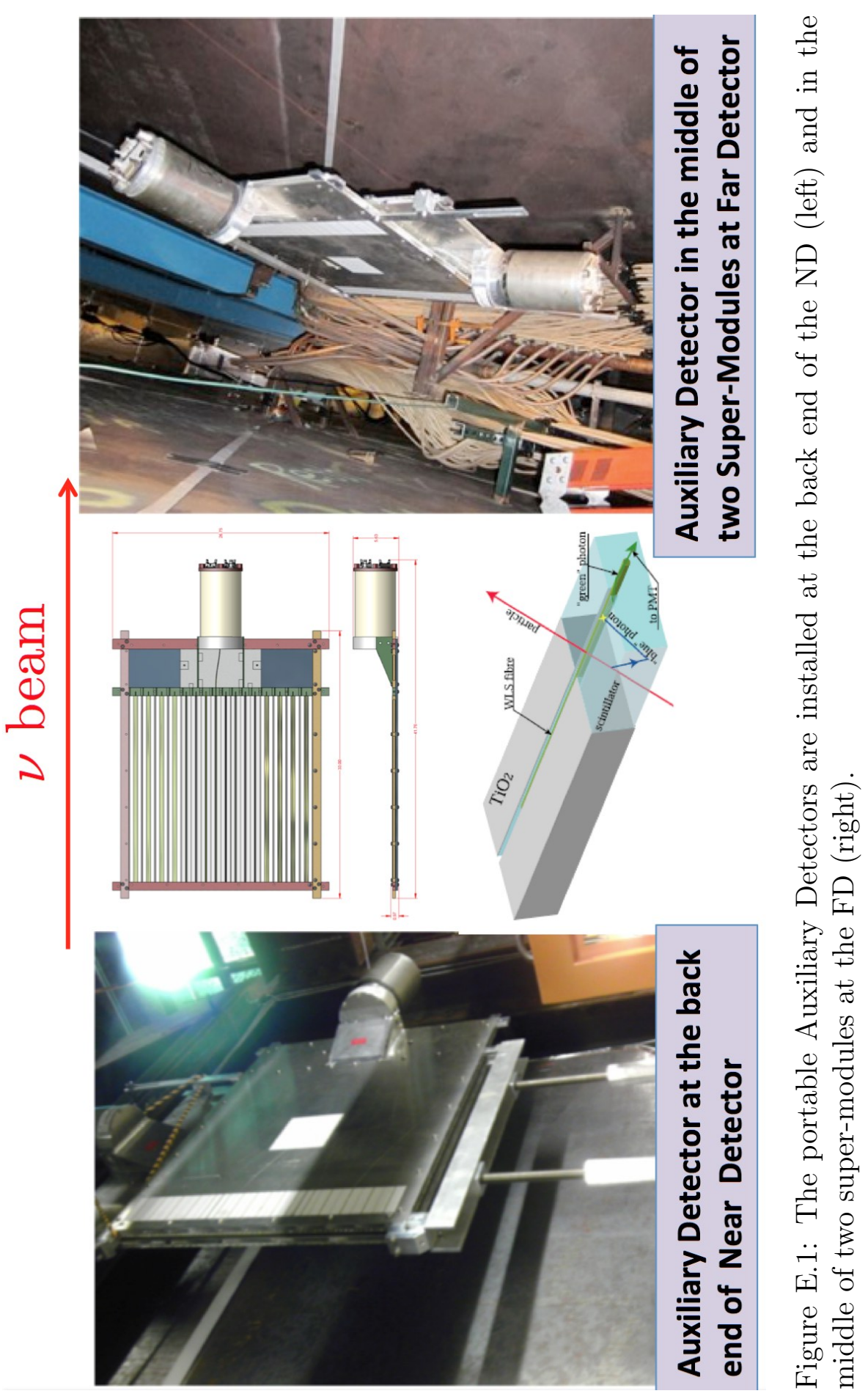




\section{Brilliant Instrument}

The Brilliant Instrument time interval analyzers (BIs), which provide a high precision time and frequency measurements with a time resolution up to $8 \mathrm{ps}$, are installed at both detectors. The BI at the ND site is used to make a cross-check with CAMAC TDC. At the FD site, the BI is the only measurement we used for time-stamping the muons since the CAMAC TDC does not help here due to the dynamic range.

These readout systems are tested and calibrated with regular pulses which mimic a PMT signal to make sure that the systems perform as expected. The AD internal electronic latency, including fiber, PMT and cookie, is estimated to be $23 \mathrm{~ns}$ [144]. This values is added up to the relative ADMINOS detector latency measurement obtained by matching muon signals to get the absolute electronic latencies of the MINOS detectors.

\section{E.3 Matching muons between the AD and MINOS de- tector}

The muon matching could be divided into two categories: beam structure and event-by-event.

\section{Beam structure matching}

This matching is implemented only at the ND site. The AD at the ND site uses the SGATE signal, derived from the NuMI beam-synchronous 
accelerator clock $\$ 74^{3}$, to trigger the muon hit recording. Figure E.2 shows distinctly the batch and bunch structures of the NuMI beam in the recorded data by the AD. Since the ND timing system tags muons respectively to the $\$ 74$ signal, the beam structure matching estimates the offset between $\$ 74$ and the SGATE signal. Figure E.3 shows the matched structures between recorded data in the AD and ND and the log-likelihood profile as a function of the offset between SGATE and $\$ 74$.

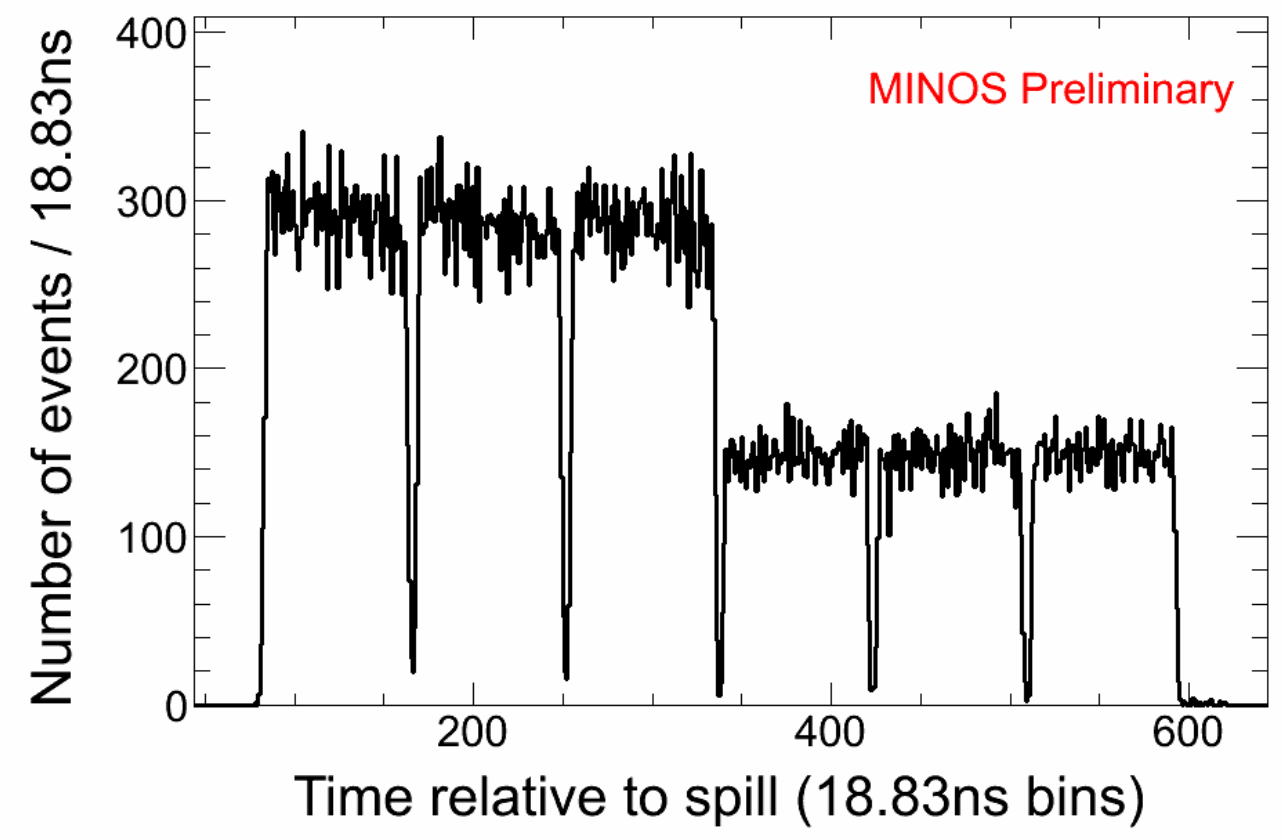

Figure E.2: Six batches beam structure observed by the Auxiliary Detector at the Near site. The $\mathrm{x}$ axis was shifted by some constant offset.

\footnotetext{
${ }^{3}$ This signal informs that protons, extracted from the NuMI, are on queue to fire.
} 

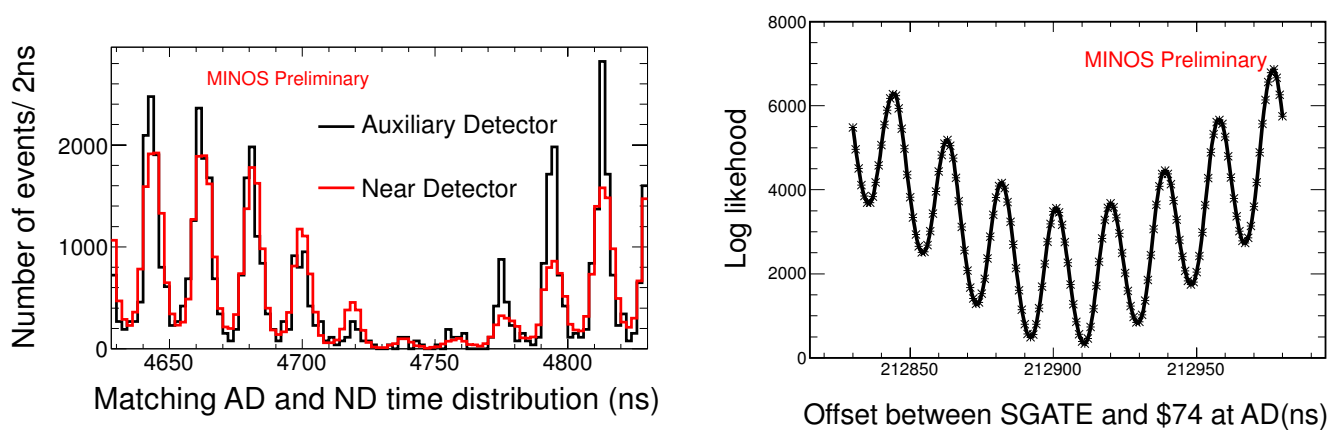

Figure E.3: The bucket structure observed by the AD and distribution matching between the AD and ND (left) and and the log likelihood of matching between the AD and ND as the function of the offset between SGATE and $\$ 74$ at ND (right). The interval between two peaks is about 19 ns which reflects very well the bucket structure of beam.

\section{Event-by-event matching}

This matching, a main method for estimating final electronic latencies, is implemented at both sites. Two straightforward conditions are utilized for this matching: (i) matched events should be in time and (ii) muons recorded in the MINOS detector should pass through the AD locations. The performances of event-by-event matching at the ND and FD sites are not the same. The following highlights the matching approaches in the two sites:

\section{Matching at the ND site}

Event-by-event matching is straightforward and it essentially measures the offset between the $\$ 74$ and SGATE signals. This offset is in the order of $212 \mu s$ which is programmed to open the SGATE whenever the $\$ 74$ signal is received. However, this offset value from matching takes into account 
the AD-ND relative electronic latency. To obtain the value of AD-ND relative electronic latency, we performed a direct measurement between these two signals. Figure E.4a shows the distribution of latency-included offset between the $\$ 74$ and the the SGATE signals from the event-by-event matching between the AD and ND data. The distribution of a pure $\$ 74$-SGATE offset from the compensational measurement is shown in Figure E.4b. The interval of $19 \mathrm{~ns}$ between the two peaks agrees with the direct measurement of the offset between the SGATE and $\$ 74$, and this is due to the jitter of the SGATE which is programed to line up with the $53.1 \mathrm{MHz}$ clock.

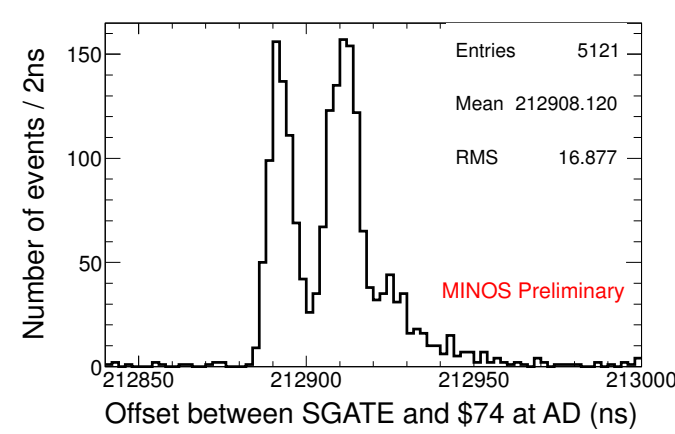

(a)

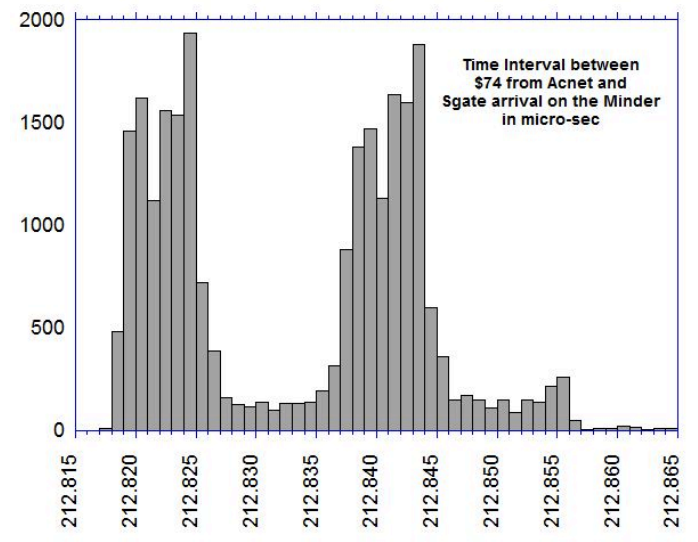

(b)

Figure E.4: The distribution of latency-included offset between the $\$ 74$ and SGATE from the event-by-event matching between AD and ND data (left) and the direct measurement of the latency-excluded offset between $\$ 74$ and SGATE (right). 


\section{Matching at the FD site}

The event-by-event matching is implemented by using the cosmic muons data recorded in the FD and that passed through the AD location. The recorded events at the FD are time-stamped using the TrueTime Pulse-perSecond (PPS) from the GPS receiver, while the AD uses the Cs clock PPS to trigger a recording of passing-through muons. Thus, to perform the eventby-event matching, some additional offset is included to make sure that two measurements using two separate timing system are in the same reference frame [145]. Figure E.5 shows the distribution of relative latency-included offset between the AD and FD from the event-by-event matching method. This distribution includes a number of corrections which take into account the difference of timing reference in which the FD and AD events are time-stamped. The right tail of the distribution is due to the noise in the coincidence unit.

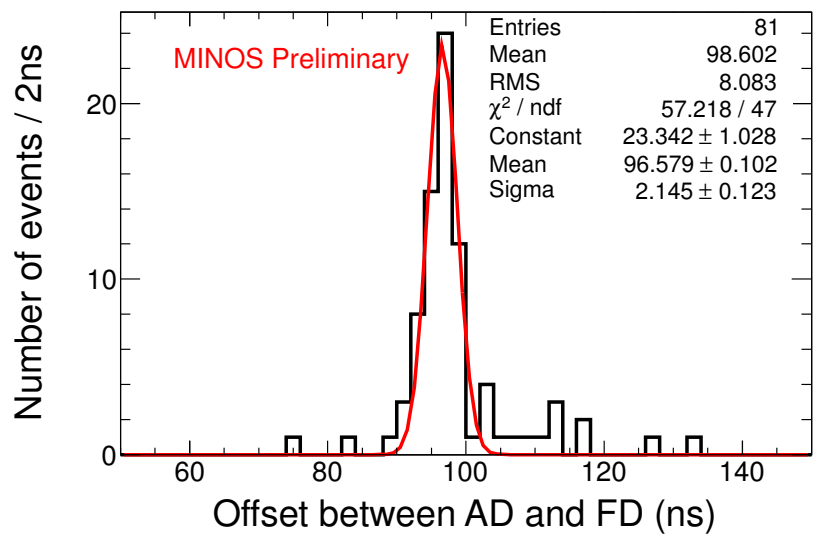

Figure E.5: The distribution of relative latency-included offset between the $\mathrm{AD}$ and Far Detector from the event-by-event matching. The cosmic muons are used for this matching. 


\section{E.4 Results of latency measurements}

Results at ND site: By comparing two beam profiles, as shown in Figure E.4, the event-by-event matching, and the direct measurement of the time interval between $\$ 74$ and SGATE; the ND-AD relative electronic latency is estimated to be $36 \mathrm{~ns}$. The uncertainty for this estimation is approximately $4 \mathrm{~ns}$, which mainly originated from the CAMAC readout system's internal clock (125 MHz) instability [146].

Results at FD site: The event-by-event matching, as depicted in Figure E.5, reveals the sharp peak with a width of $2 \mathrm{~ns}$. After taking into account cable length corrections, the FD-AD relative electronic latency is estimated to be $12 \mathrm{~ns}$. The measurement uncertainty for this is $2 \mathrm{~ns}$ [145]. By subtracting these two numbers, the relative ND-FD electronic latency is measured to be $24 \pm 1$ ns. Since the AD has simple electronics, it enables one to estimate the absolute electronic latencies. The relative ND- FD latency and the absolute electronic latencies of two detectors are used to estimate the overall time propagation of neutrinos between the two detectors.

\section{E.5 Results of neutrino velocity measurement}

In this section, the measurements of distance and time propagation of selected events are discussed. The final result of neutrino velocity measurement in the MINOS experiment is derived. 


\section{Event selection}

Two samples of candidate $\nu_{\mu}$-CC interactions are selected for this measurement. The first sample, named "contained $\nu_{\mu}$-CC", includes $\nu_{\mu}$-CC events that interact in the fiducial region. It is selected by the standard CC selection which is discussed in Chapter 4. The second one, named "rock and anti-fiducial" (RAF), consists of $\nu_{\mu}$-CC events that interact out of the fiducial region or in the rock surrounding the cavern.

\section{Distance measurement}

The distances between the MI60 and the ND, and between the ND and the FD, are measured by the Fermilab PPD Alignment \& Metrology group. The straight-line distance between the front faces of the ND and FD is reported to be $734,286.8 \pm 0.5 \mathrm{~m}$, corresponding to the ND-FD time of flight of $2,449,316.3 \pm 2.3 \mathrm{~ns}$ at the speed of light ${ }^{4}$. Almost all of the uncertainty of this measurement comes from the initial investigation of the FD location.

\section{Time propagation}

Information obtained at three separate locations is used to calculate the neutrino speed (i) the resistive wall current monitor in the NuMI beam line at MI6 (RWCM) ${ }^{5}$, (ii) the ND, and (iii) the FD. Figure E.6 depicts the layout of the time system used for neutrino velocity measurement in MINOS.

\footnotetext{
${ }^{4}$ This included the Sagnac correction.

${ }^{5}$ To time the start of proton beam
} 


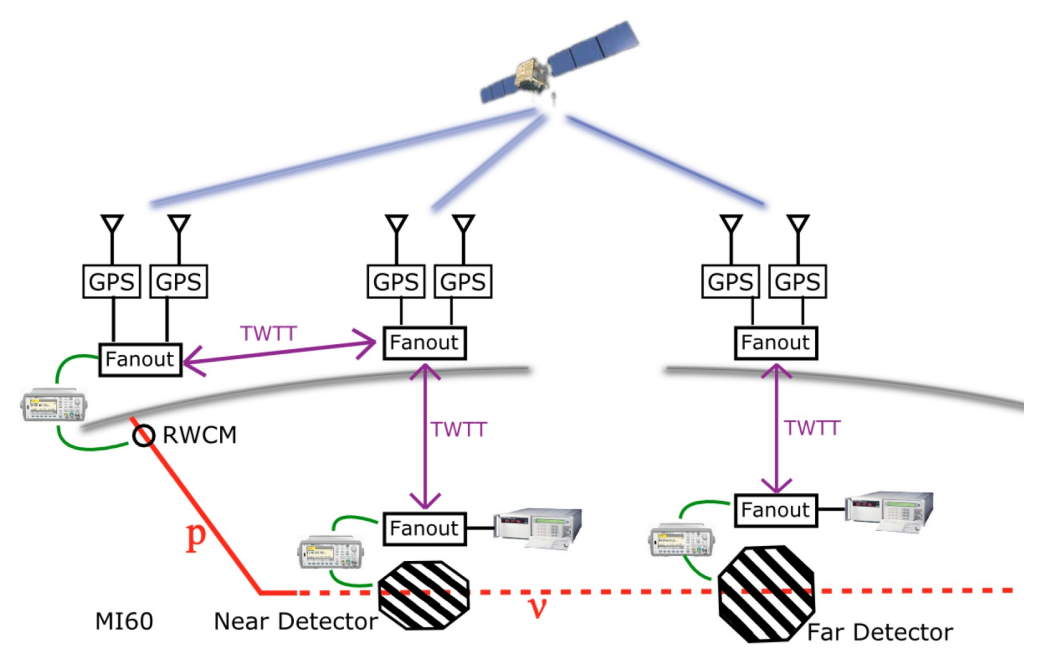

Figure E.6: Layout of the main time synchronization components of the MINOS experiment.

At each of the three locations, a free-runing atomic $\operatorname{clock}^{6}$ is used as the local source of time. The signals at each location are time-stamped respectively to this clock by an Agilent 53230A Time Interval Counter. Additionally, the two GPS receivers are installed on the surface at each location to transfer the time between them. The two-way time transfer (TWTT) systems use the fiber links that are installed between the RWCM and the ND. The ND and FD time references are underground and are transferred to secondary references on the surface using TWTT fiber links. The fiber links transfer the 1PPS and $10 \mathrm{MHz}$ signals independently. Figure E.7 shows the stability of the time-offlight measurement between the RWCM the ND with the GPS and the TWTT timing systems.

\footnotetext{
${ }^{6} \mathrm{SRS} 725 \mathrm{Rb}$ clock at the MI60, HP 5071 Cs clock at the MINOS sites
} 


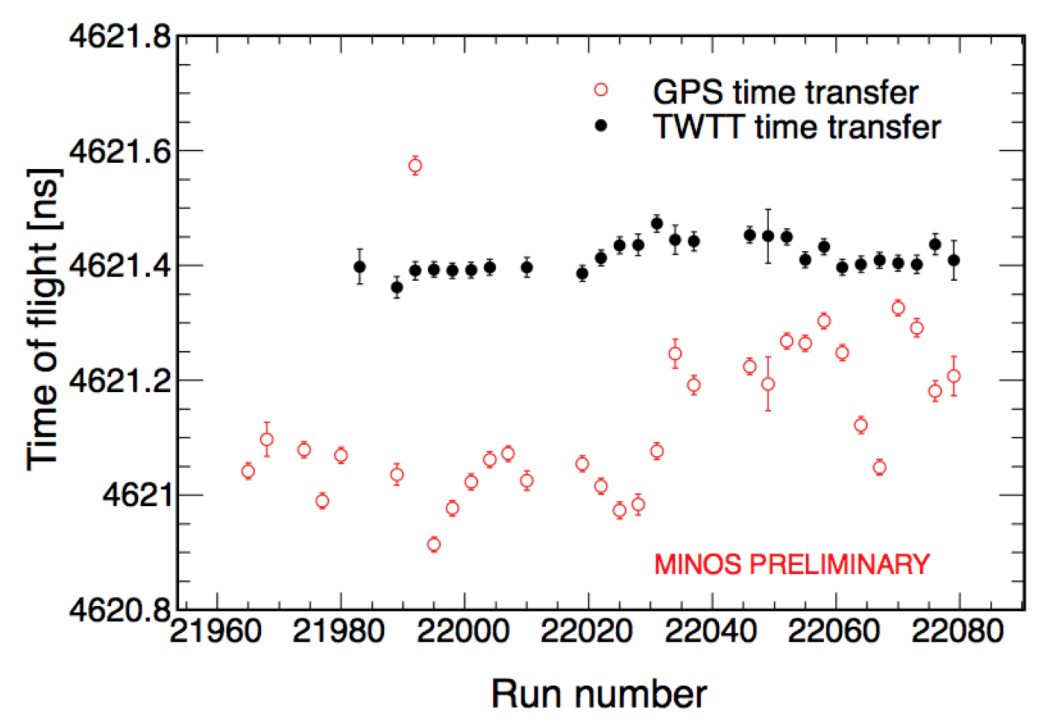

Figure E.7: Daily variation in the time propagation between the RWCM and the Near Detector using GPS and TWTT timing systems.

Comparing to $200 \mathrm{ps}$ systematic uncertainty of the GPS time transfer, the TWTT time transfer shows stability better than 50 ps.

For consistency with the measurement at the FD, the $4621.1 \mathrm{~ns}$ timeof-flight between RWCM and the ND is used. This value is consistent with the time-of-flight, $4622.7 \pm 4 \mathrm{~ns}$, estimated by using the distance between RWCM and the ND and the absolute latency of the ND measured with the AD. The uncertainty in this estimation comes from the absolute latency of the AD.

\section{Time structure of neutrino beam}

The primary $120 \mathrm{GeV}$ protons from the Main Injector accelerator have a $53 \mathrm{MHz}$ RF structure. Each proton spill lasts for approximately $10 \mu s$ and consists of six "batches". Each $1.6 \mu s$-long batch, separated by about $100 \mathrm{~ns}$, 
consists of 81 bunches of $18.83 \mathrm{~ns}$ length. The neutrino beam, produced from the decays of $\pi^{ \pm}$and $K^{ \pm}$which are created in the interactions of protons with the target, inherits the time structure from the primary $120 \mathrm{GeV}$ protons. To see this time structure, the time of flight between any two locations is plotted in the 18.83 modulo of the Main Injector bunch spacing. Figure E.8 shows the bunch shapes which are observed at the MI60 RWCM and the ND. This means that time distribution of the ND data is consistent with prediction.
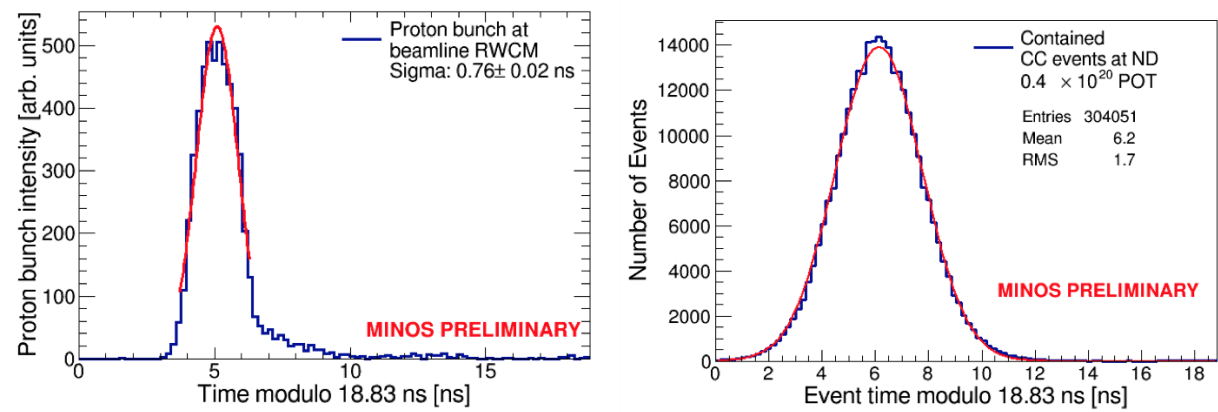

Figure E.8: The bunch structures are observed at the MI60 RWCM and the Near Detector.

\section{Results}

At the FD, we select 195 fully contained and 177 partially contained charged-current events. The separate arrival time distributions at the FD for these selected events are shown in Figure E.9. The time of flight of selected events is plotted in the $18.83 \mathrm{~ns}$ module of the MI bunch space. The bunch structure observed in the FD agreed with the ND bunch structure (see Figure E.8), which was one method of validating the timing system performance. The RWCM-ND time of flight is estimated to be 2, 453, $935 \pm 0.1 \mathrm{~ns}$. 
Subtracting $4621.1 \mathrm{~ns}$ of the RWCM-ND time of flight, yields a ND-FD time propagation as 2, 449,313.9 $\pm 1 \mathrm{~ns}$. Comparing with the prediction with speed of light, $2,449,316.3 \pm 2.3 \mathrm{~ns}$, the difference in arrival time of the neutrino and the expected light propagation is:

$$
\delta t=t_{\nu}-t_{c}=-2.4 \pm 0.1 \text { (stat.) } \pm 2.6 \text { (syst.) ns. }
$$

Neutrino velocity in comparison to the speed of light is therefore found to be:

$$
v / c-1=(1.0 \pm 1.1) \times 10^{-6} .
$$

The $2.6 \mathrm{~ns}$ systematic uncertainty is dominated by a $2.3 \mathrm{~ns}$ uncertainty from an inertial survey at the Far Detector and a $1.0 \mathrm{~ns}$ from the relative ND-FD latency measured by the ADs. The minor systematic uncertainties are $0.6 \mathrm{~ns}$ of the Far Detector TWTT fiber links and 0.5 ns of the GPS time-transfer accuracy.

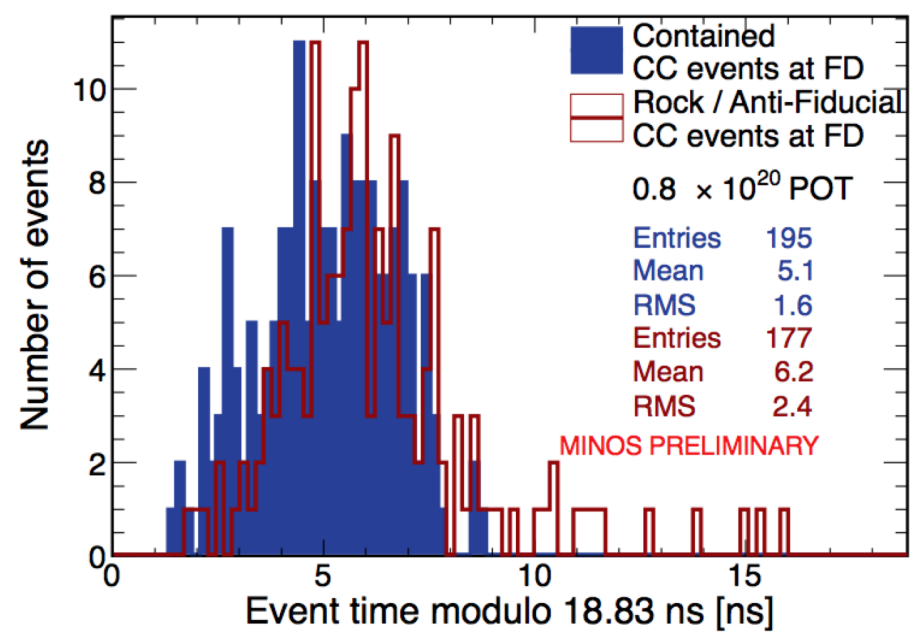

Figure E.9: The arrival time distribution at the FDr modulo the $18.83 \mathrm{~ns}$ bunch separating for the fully contained (solid) and partially contained (line) charged-current events. 


\section{E.6 Summary}

The ADs were installed to measure precisely the ND-FD relative electronic latency. The beam structure matching, which showed the batch and bunch structure of neutrino beam, confirmed that timing system in AD performed as expected. The event-by-event matching gave us precisely $24 \pm 1 \mathrm{~ns}$ of relative ND-FD latency. This is compared to 9 ns uncertainty from the previous measurement by MINOS itself. Given the fact that the AD electronics are fairly straightforward, the absolute electronic latencies in the MINOS detector were derived and showed good agreement with other methods. We

finally obtained a result of neutrino velocity, $v / c-1=(1.0 \pm 1.1) \times 10^{-6}$, which is consistent with the speed of light. 


\section{Appendix F}

\section{Unitarity of the PMNS matrix}

The unitary condition of the matrix in Eq. (1.18) leads to a number of equations:

$$
\begin{gathered}
U^{\dagger} U=U U^{\dagger}=\mathbb{1}, \\
\rightarrow U_{j i}^{*} U_{j k}=U_{i j} U_{k j}^{*}=\delta_{i k} .
\end{gathered}
$$

Eq. (F.1) gives the normalization of matrix elements. Eq. (F.2) yields six unitary triangles, given by:

$$
\begin{gathered}
U_{e 1}^{*} U_{\mu 1}+U_{e 2}^{*} U_{\mu 2}+U_{e 3}^{*} U_{\mu 3}=0 \\
U_{e 1}^{*} U_{\tau 1}+U_{e 2}^{*} U_{\tau 2}+U_{e 3}^{*} U_{\tau 3}=0 \\
U_{\mu 1}^{*} U_{\tau 1}+U_{\mu 2}^{*} U_{\tau 2}+U_{\mu 3}^{*} U_{\tau 3}=0 \\
U_{e 1} U_{e 2}^{*}+U_{\mu 1} U_{\mu 2}^{*}+U_{\tau 1} U_{\tau 2}^{*}=0 \\
U_{e 1} U_{e 3}^{*}+U_{\mu 1} U_{\mu 3}^{*}+U_{\tau 1} U_{\tau 3}^{*}=0 \\
U_{e 2} U_{e 3}^{*}+U_{\mu 2} U_{\mu 3}^{*}+U_{\tau 2} U_{\tau 3}^{*}=0
\end{gathered}
$$

These six unitary triangles have the same area, which is equal to a half of Jarlskog's invariant [147], which is defined as follows:

$$
J_{\mathrm{CP}}=\Im\left(U_{\alpha i} U_{\beta j} U_{\alpha j}^{*} U_{\beta i}^{*}\right) \text { with } \alpha \neq \beta, i \neq j
$$


We consider a specific expression, for example:

$$
U_{e 1}^{*} U_{\mu 1}+U_{e 2}^{*} U_{\mu 2}+U_{e 3}^{*} U_{\mu 3}=0
$$

If $U_{e 2}^{*} U_{\mu 2} \neq 1$, we define a new coordinate $(\rho, \eta)$ as follows:

$$
\frac{U_{e 1}^{*} U_{\mu 1}}{U_{e 2}^{*} U_{\mu 2}}=\rho+i \eta, \quad \frac{U_{e 3}^{*} U_{\mu 3}}{U_{e 2}^{*} U_{\mu 2}}=\rho-1+i \eta
$$

Apparently, the relationship Eq. (F.3) can be depicted by a triangle in twodimension coordinate, as illustrated in Figure F.1.

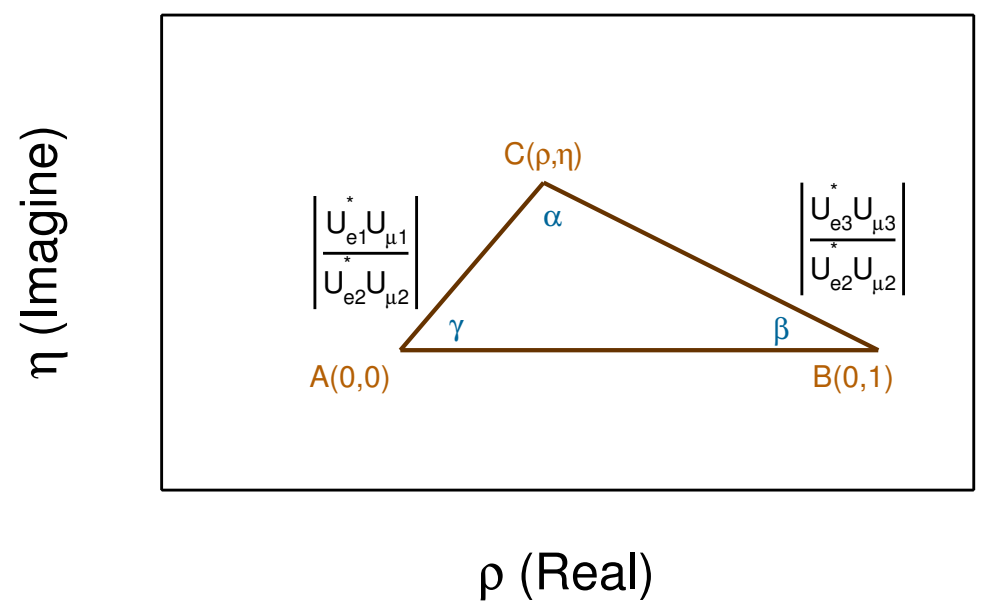

Figure F.1: A triangle is used to represents Eq. (F.3).

To determine which unitary triangles is best epresented, the experimental measurements should be considered. Up to the present day, the sources used to explore neutrino oscillation are $\nu_{e}$ and $\nu_{\mu} ; \nu_{\tau}$ involvement is limited. Thus, the denominator such as $U_{e 2}^{*} U_{\mu 2}$ in the above example should be the most precise measurement which will mitigate the uncertainty of the representing unitary triangle. From [148], based on data before (28 June 2012), the 
mixing angles are fitted by:

$s_{12}^{2}=0.307, s_{23}^{2}=\left\{\begin{array}{ll}0.386, & (\mathrm{NH}) \\ 0.392, & (\mathrm{IN})\end{array}, s_{13}^{2}=\left\{\begin{array}{ll}0.0241, & (\mathrm{NH}) \\ 0.0244, & (\mathrm{IN})\end{array}, \delta_{\mathrm{CP}}= \begin{cases}1.08 \pi, & (\mathrm{NH}) \\ 1.09 \pi, & (\mathrm{IN})\end{cases}\right.\right.$

By using these values, the PMNS matrices (for normal and invert hierarchies) are calculated by:

$$
U_{\text {normal }}=\left(\begin{array}{ccc}
0.822 & 0.547 & -0.150+0.039 * i \\
-0.356+0.020 * i & 0.704+0.013 * i & 0.614 \\
0.442+0.025 * i & -0.452+0.017 * i & 0.774
\end{array}\right)
$$

and

$$
U_{\text {invert }}=\left(\begin{array}{ccc}
0.822 & 0.547 & -0.150+0.044 * i \\
-0.354+0.023 * i & 0.701+0.015 * i & 0.618 \\
0.444+0.028 * i & -0.456+0.019 * i & 0.770
\end{array}\right) .
$$

The unitary triangle representations for the normal and inverted hierarchies are shown in Figure F.2.

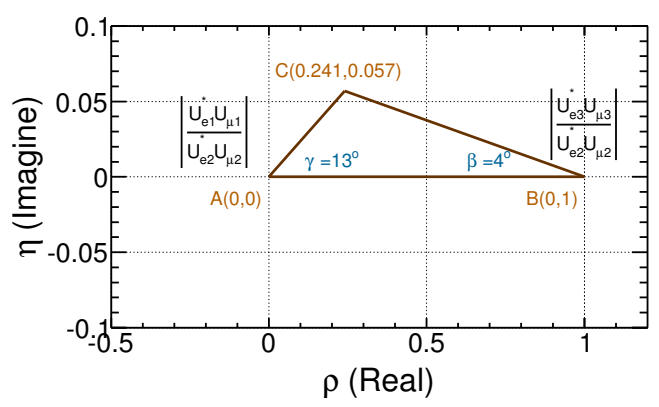

(a) Normal hierarchy

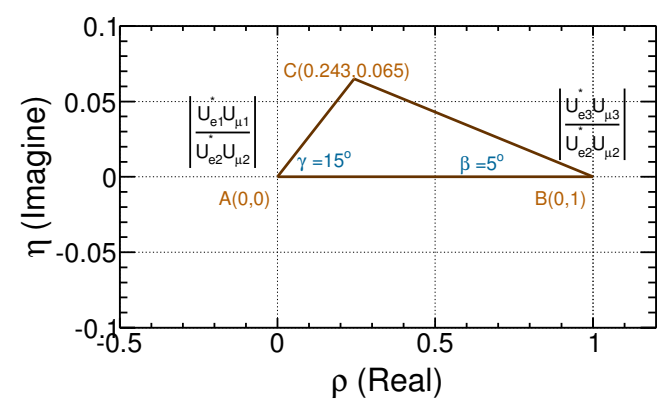

(b) Inverted hierarchy

Figure F.2: Unitary triangles with the best-fit values of mixing angles from global analysis. The normal hierarchy (left) and the inverted hierarchy (right) are calculated independently. All angles lie in the first quadrant $(\theta<\pi / 2)$ are assumed. 


\section{Simple model for estimating uncertainty}

The goal is to estimate the confidence interval (or credible interval in Bayesian approach) for the $C$ vertex in each unitary triangle.

Error propagation: Theoretically, it is possible to calculate the uncertainties of coordinates of $C$ vertex in the unitary triangle. But it is fairly complicated when you need to take into account the complex numbers from CP phase.

Bayesian approach: The straightforward way to deal with uncertainty is to assume Gaussian distribution for each parameter (a kind of Bayesian approach). Then, we generate Gaussian random variables for three angles and one CP phase. The distribution of each of the PMNS elements, as well as the coordinates of $C$ vertex is calculated directly. Figure F.3 shows the unitary triangle representation with the uncertainty of the $C$ vertex's coordinates.
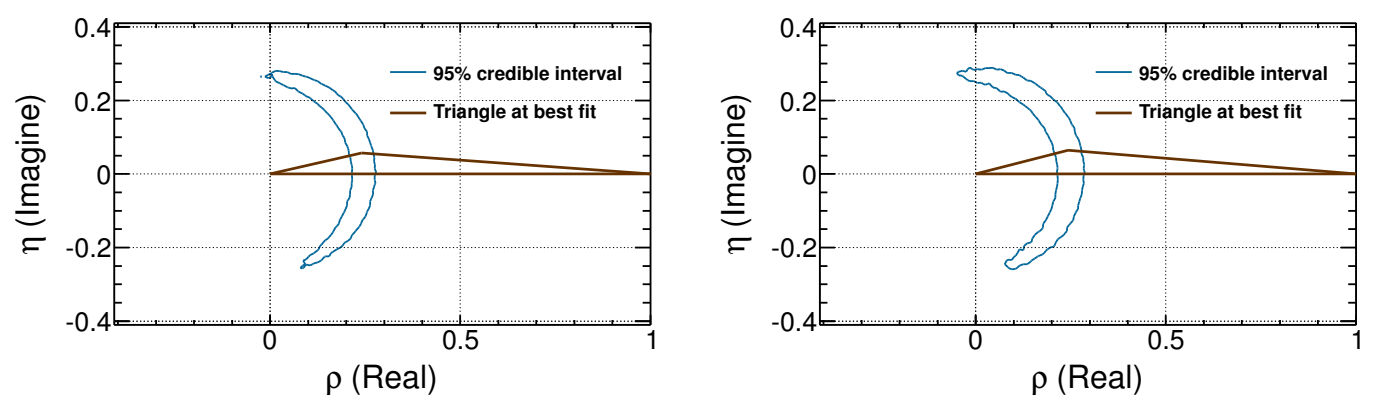

Figure F.3: The unitary triangle and 95\% credible interval of the $C$ vertex's coordinates (Figure F.2) assuming normal (left) and inverted (right) hierarchy. 


\section{Bibliography}

[1] W. Pauli. Letter to Tübingen Conference, December 1930.

[2] P. Adamson et al., "First direct observation of muon antineutrino disappearance," Phys.Rev.Lett., vol. 107, no. 021801, 2011.

[3] J. Chadwick, "Distribution in intensity in the magnetic spectrum of the $\beta$-rays of radium," Ver. Dtsch. Physik. Ges., vol. 16, pp. 383-391, 1914.

[4] C. Cowan, F. Reines, F. Harrison, H. Kruse, and A. McGuire, "Detection of the free neutrino: A Confirmation," Science, vol. 124, pp. 103-104, 1956.

[5] I. V. Anicin, "The Neutrino: Its past, present and future," arXiv:physics/0503172, 2005.

[6] G. Danby, J. Gaillard, K. A. Goulianos, L. Lederman, N. B. Mistry, et al., "Observation of high-energy neutrino reactions and the existence of two kinds of neutrinos," Phys.Rev.Lett., vol. 9, pp. 36-44, 1962.

[7] M. Perl et al., "Evidence for anomalous lepton production in $\mathrm{e}^{+} \mathrm{e}^{-}$ annihilation," Phys.Rev.Lett., vol. 35, pp. 1489-1492, 1975. 
[8] K. Kodama et al., "Observation of tau neutrino interactions," Phys.Lett., vol. B504, pp. 218-224, 2001.

[9] J. Beringer et al., "Review of particle physics (rpp)," Phys.Rev., vol. D86, p. 010001, 2012.

[10] S. Schael et al., "Precision electroweak measurements on the $z$ resonance," Phys.Rept., vol. 427, pp. 257-454, 2006.

[11] P. Ade et al., "Planck 2013 results. xvi. cosmological parameters," arXiv, 2013.

[12] B. Pontecorvo, "Mesonium and anti-mesonium," Sov.Phys.JETP, vol. 6, p. 429, 1957.

[13] Y. Fukuda et al., "Evidence for oscillation of atmospheric neutrinos," Phys.Rev.Lett., vol. 81, pp. 1562-1567, 1998.

[14] D. Michael et al., "Observation of muon neutrino disappearance with the MINOS detectors and the NuMI neutrino beam," Phys.Rev.Lett., vol. 97, p. 191801, 2006.

[15] Q. Ahmad et al., "Measurement of the rate of $\nu_{e}+d \rightarrow p+p+e^{-}$ interactions produced by ${ }^{8} b$ solar neutrinos at the Sudbury Neutrino Observatory," Phys.Rev.Lett., vol. 87, p. 071301, 2001.

[16] K. Eguchi et al., "First results from KamLAND: Evidence for reactor anti-neutrino disappearance," Phys.Rev.Lett., vol. 90, p. 021802, 2003. 
[17] D. M. Webber et al., "An improved measurement of electron antineutrino disappearance at Daya Bay," Nucl.Phys.Proc.Suppl., vol. 233, pp. 96-101, 2012.

[18] K. Abe et al., "Observation of electron neutrino appearance in a muon neutrino beam," Phys.Rev.Lett., vol. 112, p. 061802, 2014.

[19] B. Kayser and G. Segre, "Leptogenesis at the electroweak scale," Phys.Lett., vol. B704, pp. 570-573, 2011.

[20] A. P. Society et al., "The neutrino matrix," APS, 2004.

[21] E. Fermi, "An attempt of a theory of beta radiation. 1," Z.Phys., vol. 88, pp. 161-177, 1934.

[22] E. Commins and P. Bucksbaum, "Weak interactions of leptons and quarks," Cambridge University Press, 1983.

[23] A. Salam and J. C. Ward, "Electromagnetic and weak interactions," Phys.Lett., vol. 13, pp. 168-171, 1964.

[24] S. Glashow, "Partial symmetries of weak interactions," Nucl.Phys., vol. 22, pp. 579-588, 1961.

[25] S. Weinberg, "A model of leptons," Phys.Rev.Lett., vol. 19, pp. 1264-1266, 1967.

[26] C. Giunti and C. W. Kim, Fundamentals of Neutrino Physics and Astrophysics. Oxford University Press, 2007. 
[27] F. P. B Kayser, F Gibrat-Debu, The Physics Of Massive Neutrinos, vol. 25. World Scientific Lecture Notes in Physics, 1989.

[28] K. Lodders, "Solar system abundances and condensation temperatures of the elements," Astrophys.J., vol. 591, pp. 1220-1247, 2003.

[29] J. Davis, Raymond, D. S. Harmer, and K. C. Hoffman, "Search for neutrinos from the sun," Phys.Rev.Lett., vol. 20, pp. 1205-1209, 1968.

[30] W. Hampel et al., "GALLEX solar neutrino observations: Results for GALLEX IV," Phys.Lett., vol. B447, pp. 127-133, 1999.

[31] M. Altmann et al., "Complete results for five years of GNO solar neutrino observations," Phys.Lett., vol. B616, pp. 174-190, 2005.

[32] J. Abdurashitov et al., "Solar neutrino flux measurements by the Soviet-American Gallium Experiment (SAGE) for half the 22 year solar cycle," J.Exp.Theor.Phys., vol. 95, pp. 181-193, 2002.

[33] B. Aharmim et al., "Electron energy spectra, fluxes, and day-night asymmetries of b-8 solar neutrinos from measurements with nacl dissolved in the heavy-water detector at the Sudbury Neutrino Observatory," Phys.Rev., vol. C72, p. 055502, 2005.

[34] Y. Fukuda et al., "Solar neutrino data covering solar cycle 22," Phys.Rev.Lett., vol. 77, pp. 1683-1686, 1996. 
[35] Y. Fukuda et al., "Measurements of the solar neutrino flux from Super-Kamiokande's first 300 days," Phys.Rev.Lett., vol. 81, pp. 1158-1162, 1998.

[36] A. Serenelli, "Standard Solar Model: Status and prospects," Nucl.Phys.Proc.Suppl., vol. 235-236, pp. 41-48, 2013.

[37] J. M. Conrad, "Recent results on neutrino oscillations," hep-ex/9811009, 1998.

[38] M. Aglietta et al., "Experimental study of atmospheric neutrino flux in the NUSEX experiment," Europhys.Lett., vol. 8, pp. 611-614, 1989.

[39] K. Hirata et al., "Observation of a small atmospheric muon-neutrino / electron-neutrino ratio in Kamiokande," Phys.Lett., vol. B280, pp. 146-152, 1992.

[40] R. Becker-Szendy, C. Bratton, D. Casper, S. Dye, W. Gajewski, et al., "The electron-neutrino and muon-neutrino content of the atmospheric flux," Phys.Rev., vol. D46, pp. 3720-3724, 1992.

[41] K. Daum et al., "Determination of the atmospheric neutrino spectra with the Frejus detector," Z.Phys., vol. C66, pp. 417-428, 1995.

[42] S. Ahlen et al., "Atmospheric neutrino flux measurement using upgoing muons," Phys.Lett., vol. B357, pp. 481-486, 1995. 
[43] W. Allison, G. Alner, D. Ayres, W. Barrett, C. Bode, et al., "Measurement of the atmospheric neutrino flavor composition in Soudan-2," Phys.Lett., vol. B391, pp. 491-500, 1997.

[44] Z. Maki, M. Nakagawa, and S. Sakata, "Remarks on the unified model of elementary particles," Prog.Theor.Phys., vol. 28, pp. 870-880, 1962.

[45] B. Pontecorvo, "Electron and muon neutrinos," Sov.Phys.JETP, vol. 10, pp. 1236-1240, 1960.

[46] J. Ahn et al., "Observation of reactor electron antineutrino disappearance in the RENO experiment," Phys.Rev.Lett., vol. 108, p. 191802, 2012.

[47] Y. Abe et al., "Indication for the disappearance of reactor electron antineutrinos in the Double Chooz experiment," Phys.Rev.Lett., vol. 108, p. 131801, 2012.

[48] P. Adamson et al., "A Study of Muon Neutrino Disappearance Using the Fermilab Main Injector Neutrino Beam," Phys.Rev.D, vol. D77, p. $072002,2008$.

[49] K. Abe et al., "Precise measurement of the neutrino mixing parameter $\theta_{23}$ from muon neutrino disappearance in an off-axis beam," arXiv hep-ex/1403.1532, 2014.

[50] R. Patterson et al., "The NOvA experiment: Status and outlook," Nucl.Phys.Proc.Suppl., vol. 235-236, pp. 151-157, 2013. 
[51] P. Adamson et al., "Combined analysis of $\nu_{\mu}$ disappearance and $\nu_{\mu} \rightarrow \nu_{e}$ appearance in MINOS using accelerator and atmospheric neutrinos," arXiv hep-ex/1403.0867, 2014.

[52] L. Wolfenstein, "Neutrino oscillations in matter," Phys.Rev., vol. D17, pp. 2369-2374, 1978.

[53] S. Mikheev and A. Y. Smirnov, "Resonance amplification of oscillations in matter and spectroscopy of solar neutrinos," Sov.J.Nucl.Phys., vol. 42, pp. 913-917, 1985.

[54] J. A. A. Boehm, Measurement of electron neutrino appearance with the MINOS experiment. PhD thesis, Harvard University, 2009.

[55] H. Nunokawa, S. J. Parke, and R. Zukanovich Funchal, "Another possible way to determine the neutrino mass hierarchy," Phys.Rev., vol. D72, p. 013009, 2005.

[56] V. D. Barger, J. Learned, S. Pakvasa, and T. J. Weiler, "Neutrino decay as an explanation of atmospheric neutrino observations," Phys.Rev.Lett., vol. 82, pp. 2640-2643, 1999.

[57] E. Lisi, A. Marrone, and D. Montanino, "Probing possible decoherence effects in atmospheric neutrino oscillations," Phys.Rev.Lett., vol. 85, pp. 1166-1169, 2000.

[58] J. Mitchell, Measuring $\nu_{\mu}$ Disappearance with the MINOS Experiment. PhD thesis, Cambridge University, 2011. 
[59] P. Machado, H. Nunokawa, and R. Zukanovich Funchal, "Testing for large extra dimensions with neutrino oscillations," Phys.Rev., vol. D84, p. 013003, 2011.

[60] P. Adamson et al., "Active to sterile neutrino mixing limits from neutral-current interactions in MINOS," Phys.Rev.Lett., vol. 107, no. $011802,2011$.

[61] P. Adamson et al., "A search for flavor-changing non-standard neutrino interactions by MINOS," Phys.Rev., vol. D88, p. 072011, 2013.

[62] P. Adamson et al., "A search for Lorentz invariance and CPT Violation with the MINOS Far Detector," Phys.Rev.Lett., vol. 105, 2010.

[63] S. Hollenberg, O. Micu, and H. Pas, "Neutrino-antineutrino oscillations as a possible solution for the LSND and MiniBooNE anomalies?," Phys.Rev., vol. D80, p. 053010, 2009.

[64] K. Hirata et al., "Observation of a neutrino burst from the supernova SN 1987a," Phys.Rev.Lett., vol. 58, pp. 1490-1493, 1987.

[65] J. C. V. D. Velde et al., "Neutrinos from SN1987a in the IMB detector," Nucl. Instrum. Meth., vol. A264, pp. 28-31, 1988.

[66] J. N. Bahcall, A. M. Serenelli, and S. Basu, "New solar opacities, abundances, helioseismology, and neutrino fluxes," Astrophys.J., vol. 621, pp. L85-L88, 2005. 
[67] Y. Suzuki, "Solar neutrinos," Int.J.Mod.Phys., vol. A15S1, pp. 201-228, 2000.

[68] J. Ahrens et al., "Icecube - the next generation neutrino telescope at the south pole," Nucl.Phys.Proc.Suppl., vol. 118, pp. 388-395, 2003.

[69] S. Amerio et al., "Design, construction and tests of the ICARUS T600 detector," Nucl.Instrum.Meth., vol. A527, pp. 329-410, 2004.

[70] G. Alimonti et al., "Science and technology of Borexino: A real time detector for low-energy solar neutrinos," Astropart.Phys., vol. 16, pp. 205-234, 2002.

[71] C. Kraus et al., "SNO with liquid scintillator: SNO+," Prog.Part.Nucl.Phys., vol. 57, pp. 150-152, 2006.

[72] A. McDonald, "SNO and future solar neutrino experiments," Nucl.Phys.Proc.Suppl., vol. 235-236, pp. 61-67, 2013.

[73] G. Barr, T. Gaisser, S. Robbins, and T. Stanev, "Uncertainties in atmospheric neutrino fluxes," Phys.Rev., vol. D74, p. 094009, 2006.

[74] S. Choubey, "Future of atmospheric neutrino measurements," Nucl.Phys.Proc.Suppl., vol. 235-236, pp. 87-94, 2013.

[75] S. E. Kopp, "Accelerator-based neutrino beams," Phys.Rept., vol. 439, pp. 101-159, 2007. 
[76] A. Bandyopadhyay et al., "Physics at a future neutrino factory and super-beam facility," Rept.Prog.Phys., vol. 72, p. 106201, 2009.

[77] K. T. McDonald, "An off-axis neutrino beam," arXiv, 2001.

[78] V. Antonelli, L. Miramonti, C. Pena-Garay, and A. Serenelli, "Solar neutrinos," Adv.High Energy Phys., vol. 2013, p. 351926, 2013.

[79] P. Adamson et al., "Measurement of neutrino and antineutrino oscillations using beam and atmospheric data in MINOS," arXiv hep-ex/1304.6335, 2013.

[80] K. Abe et al., "Search for differences in oscillation parameters for atmospheric neutrinos and antineutrinos at Super-Kamiokande," Phys.Rev.Lett., vol. 107, p. 241801, 2011.

[81] N. Agafonova et al., "New results on $\nu_{\mu} \rightarrow \nu_{\tau}$ appearance with the OPERA experiment in the CNGS beam," JHEP, vol. 1311, p. 036, 2013.

[82] C. Athanassopoulos et al., "Evidence for $\nu_{\mu} \rightarrow \nu_{e}$ neutrino oscillations from LSND," Phys.Rev.Lett., vol. 81, no. 1774-1777, 1998.

[83] A. Aguilar-Arevalo et al., "Improved search for $\bar{\nu}_{\mu} \rightarrow \bar{\nu}_{e}$ oscillations in the MiniBooNE experiment," Phys.Rev.Lett., vol. 110, p. 161801, 2013.

[84] G. Mention, M. Fechner, T. Lasserre, T. Mueller, D. Lhuillier, et al., "The reactor antineutrino anomaly," Phys.Rev., vol. D83, p. 073006, 2011. 
[85] W. D. Arnett and J. L. Rosner, "Neutrino mass limits from SN1987a," Phys.Rev.Lett., vol. 58, p. 1906, 1987.

[86] C. Kraus, B. Bornschein, L. Bornschein, J. Bonn, B. Flatt, et al., "Final results from phase ii of the Mainz neutrino mass search in tritium beta decay," Eur.Phys.J., vol. C40, pp. 447-468, 2005.

[87] A. Gando et al., "Limit on neutrinoless $\beta \beta$ decay of Xe-136 from the first phase of KamLAND-Zen and comparison with the positive claim in Ge-76," Phys.Rev.Lett., vol. 110, p. 062502, 2013.

[88] M. Auger et al., "Search for neutrinoless double-beta decay in ${ }^{136} \mathrm{Xe}$ with EXO-200," Phys.Rev.Lett., vol. 109, p. 032505, 2012.

[89] M. Schwartz, "Feasibility of using high-energy neutrinos to study the weak interactions," Phys.Rev.Lett., vol. 4, pp. 306-307, 1960.

[90] I. Ambats et al., "The MINOS Detectors Technical Design Report," NuMI-L-337, FERMILAB-DESIGN-1998-02, 1998.

[91] D. Casper, R. Becker-Szendy, C. Bratton, D. Cady, R. Claus, et al., "Measurement of atmospheric neutrino composition with IMB-3," Phys.Rev.Lett., vol. 66, pp. 2561-2564, 1991.

[92] M. Ambrosio et al., "Measurements of atmospheric muon neutrino oscillations, global analysis of the data collected with MACRO detector," Eur.Phys.J., vol. C36, pp. 323-339, 2004. 
[93] Y. Ashie et al., "A measurement of atmospheric neutrino oscillation parameters by Super-Kamiokande I," Phys.Rev., vol. D71, no. 112005, 2005.

[94] W. Allison et al., "Neutrino oscillation effects in Soudan-2 upward-stopping muons," Phys.Rev., vol. D72, p. 052005, 2005.

[95] R. M. Zwaska, Accelerator Systems and Instrumentation for the NuMI Neutrino Beam. PhD thesis, The University of Texas at Austin, 2005.

[96] S. van der Meer, "A directive device for charged particles and its use in an enhanced neutrino beam," CERN-61-0\%, 1961.

[97] L. J. Loiacono, Measurement of the Muon Neutrino Inclusive Charged Current Cross Section on Iron using the MINOS Detector. PhD thesis, The University of Texas at Austin, 2010.

[98] D. Michael et al., "The magnetized steel and scintillator calorimeters of the MINOS experiment," Nucl.Instrum.Meth., vol. A596, pp. 190-228, 2008.

[99] M. Campanella, A. Ferrari, P. Sala, and S. Vanini, "First calorimeter simulation with the flugg prototype," ATL-SOFT-99-004, 1999.

[100] R. Brun, F. Carminati, and S. Giani, "GEANT Detector Description and Simulation Tool," CERN-W5013, 1994. 
[101] A. Fasso, A. Ferrari, S. Roesler, P. Sala, F. Ballarini, et al., "The physics models of FLUKA: Status and recent developments," eConf, vol. C0303241, 2003.

[102] H. Gallagher, "The NEUGEN neutrino event generator," Nucl.Phys.Proc.Suppl., vol. 112, pp. 188-194, 2002.

[103] C. Zeitnitz and T. Gabriel, "The GEANT - CALOR interface and benchmark calculations of ZEUS test calorimeters," Nucl.Instrum.Meth., vol. A349, pp. 106-111, 1994.

[104] P. Ballester, "Hough transform for robust regression and automated detection," Astron.Astrophys., vol. 286, p. 1011, 1994.

[105] J. S. Marshall, A study of muon neutrino disappearance with the MINOS detectors and the NuMI neutrino beam. PhD thesis, University of Cambridge, 2008.

[106] R. Lee, "Event reconstruction in the Near Detector." NuMI-NOTE-COMP-917, 2000.

[107] G. Tzanankos et al., "MINOS+: a proposal to fnal to run MINOS with the medium energy NuMI beam," FERMILAB-PROPOSAL-1016, 2011. MINOS docdb 7923.

[108] J. P. Ochoa, Search for nue appearance with the LEM selection. PhD thesis, Caltech, 2009. 
[109] R. Ospanov, A measurement of muon neutrino disappearance with the MINOS detectors and NuMI beam. PhD thesis, The University of Texas at Austin, 2008.

[110] J. S. Ratchford, Identifying Muons for Neutrino Oscillation and Cross Section Experiments. PhD thesis, The University of Texas at Austin, 2012.

[111] J. J. Hartnell, Measurement of the MINOS Detectors' Relative Calorimetric Energy Response. PhD thesis, University of Oxford, 2005.

[112] R. T. Trevor Hastie and J. H. Friedman, The elements of statistical learning: data mining, inference, and prediction. Springer-Verlag, 2009.

[113] A. Hocker, J. Stelzer, F. Tegenfeldt, H. Voss, K. Voss, et al., "Tmva toolkit for multivariate data analysis," arXiv:physics/0703039, 2007.

[114] P. Adamson et al., "Search for the disappearance of muon antineutrinos in the NuMI neutrino beam," Phys.Rev., vol. D84, p. 071103, 2011.

[115] R. Mehdiyev, "FHC 7.1e20 numubar oscillation results wit alternative numubar event selector." MINOS docdb 8041-v1, 2011.

[116] C. Backhouse et al., "NC analysis blessing package for 7e20 pot MINOS data sample." MINOS docdb 7344-v7, 2010.

[117] S. V. Cao, "Neutral-current selection." MINOS docdb 10279-v2, 12 2013. 
[118] P. L. Vahle, Electromagnetic interactions in the MINOS detectors. PhD thesis, The University of Texas at Austin, 2004.

[119] M. A. Kordosky, Hadronic interactions in the MINOS detectors. $\mathrm{PhD}$ thesis, The University of Texas at Austin, 2004.

[120] C. J. Backhouse, Measuring neutrino oscillation parameters using $\nu_{\mu}$ disappearance in MINOS. PhD thesis, University of Oxford, 2011.

[121] T. Yang, C. Andreopoulos, H. Gallagher, K. Hoffmann, and P. Kehayias, "A hadronization model for few-gev neutrino interactions," Eur.Phys.J., vol. C63, pp. 1-10, 2009.

[122] M. Kordosky, H. Gallagher, and S. Dytman, "Estimated uncertainty on the shower energy scale in $\nu_{\mu}$-cc events." MINOS docdb 3362-v1, 2007.

[123] R. Ransome, "Pion absorption and re-scattering," Nucl.Phys.Proc.Suppl., vol. 139, pp. 208-212, 2005.

[124] S. Dytman and H. Gallagher, "Changes to the determination of the mean free path of hadrons in nuclear matter in intranuke." MINOS docdb 4358-v2, 2008.

[125] J. Boehm, H. Gallagher, and T. Yang, "Hadronization model uncertainties for the $\nu_{e}$ analysis." MINOS docdb 5392-v1, 2008.

[126] R. M. et al, "Determination of pion intranuclear rescattering rates in $\nu_{\mu}$-ne versus $\nu_{\mu}$-d interactions for the atmospheric $\nu$ flux," Phys. Rev. $D$, vol. 45, no. 3, pp. 743-751, 1992. 
[127] P. Adamson et al., "Measurements of atmospheric neutrinos and antineutrinos in the MINOS Far Detector," Phys.Rev., vol. D86, p. $052007,2012$.

[128] J. J. Evans, "Run 9 target decay update." MINOS docdb 8826-v2, 2012.

[129] K. Sacha, P. Zarko, and V. Patricia, "Fitting the beam MC to the ND data." MINOS docdb 1548-v5, 2006.

[130] J. J. Evans, Measuring Antineutrino Oscillations with the MINOS Experiment. PhD thesis, University of Oxford, 2008.

[131] S. E. Kopp, R. Ospanov, P. Zarko, and P. L. Vahle, "Constraining the beam neutrino MC flux using the MINOS ND data." MINOS docdb 2965-v1, 2007.

[132] I. Dharma, S. E. Kopp, and P. Zarko, "Systematic uncertainties in the NuMI beam flux." MINOS docdb 1283-v3, 2007.

[133] F. James and M. Roos, "Minuit: A system for function minimization and analysis of the parameter errors and correlations," Comput.Phys.Commun., vol. 10, pp. 343-367, 1975.

[134] S. V. Cao and M. Matthis, "Blessed plots for the 2012 RHC analysis." MINOS docdb 9020-v8, 052012. 
[135] G. J. Feldman and R. D. Cousins, "A unified approach to the classical statistical analysis of small signal," Phys.Rev., vol. D57, pp. 3873-3889, 1998.

[136] A. I. Himmel, Antineutrino Oscillations in the Atmospheric Sector. PhD thesis, California Institute of Technology, 2011.

[137] P. Adamson et al., "Measurement of the neutrino mass splitting and flavor mixing by MINOS," Phys.Rev.Lett, vol. 106, p. 181801, 2011.

[138] S. V. Cao, "Antineutrino combination in RHC and FHC modes." MINOS docdb 8389-v1, 072011.

[139] L. A. Corwin, "RHC \& atm. $\bar{\nu}$ combination." MINOS docdb 8489-v1.

[140] A. Blake, "Combining MINOS beam and atmospheric neutrino data." MINOS docdb 8780-v1.

[141] S. Rigolin, "Physics reach of beta-beams and nu-factories: The problem of degeneracies," Nucl.Phys.Proc.Suppl., vol. 155, pp. 33-37, 2006.

[142] V. Ammosov and G. Volkov, "Can neutrinos probe extra dimensions?," arXiv hep-ph/0008032, 2000.

[143] P. Adamson et al., "Measurement of neutrino velocity with the MINOS detectors and NuMI neutrino beam," Phys.Rev., vol. D76, p. 072005, 2007. 
[144] S. V. Cao, R. Mehdiyev, and X. Qiu, "Offset between sgate and \$74." MINOS docdb 8762-v7, 2012.

[145] S. V. Cao, R. Mehdiyev, and X. Qiu, "Blessing package on measuring electronics latencies in MINOS with Auxiliary Detectors." MINOS docdb 9228-v8, 2012.

[146] P. Adamson, S. V. Cao, and R. Mehdiyev, "ND CAMAC and BI tests." MINOS docdb 9219-v1, 2012.

[147] C. Jarlskog, "Commutator of the quark mass matrices in the standard electroweak model and a measure of maximal CP violation," Phys.Rev.Lett., vol. 55, p. 1039, 1985.

[148] G. Fogli, E. Lisi, A. Marrone, D. Montanino, A. Palazzo, et al., "Global analysis of neutrino masses, mixings and phases: entering the era of leptonic CP violation searches," Phys.Rev., vol. D86, p. 013012, 2012. 


\section{Vita}

Son Van Cao was born in Quang Binh province, Vietnam. He received the Bachelor of Science degree in Physics in 2008 from the Hanoi University of Science, Vietnam National University. He worked at the Institute of Physics as a research assistant for one year before he got the Vietnam Education Foundation fellowship and applied to the University of Texas at Austin for enrollment in their physics program. He was accepted and started graduate studies in August, 2009. He has mainly worked in the MINOS experiment at Fermilab, Batavia, IL since the beginning of his graduate research.

Permanent address: Email: cvson@utexas.edu.

This dissertation was typeset with $\mathrm{LT}_{\mathrm{E}} \mathrm{X}^{\dagger}$ by the author.

\footnotetext{
${ }^{\dagger} \mathrm{LT}_{\mathrm{E}} \mathrm{X}$ is a document preparation system developed by Leslie Lamport as a special version of Donald Knuth's TEX Program.
} 